\title{
MHD-Computersimulationen zur Begleitung des Projektes DRESDyn
}

\author{
Dissertation \\ zur Erlangung des Doktorgrades \\ "Doctor rerum naturalium" \\ der Mathematisch-Naturwissenschaftlichen Fakultäten \\ der Georg-August-Universität zu Göttingen \\ im Promotionsprogramm ProPhys \\ der Georg-August University School of Science (GAUSS)
}

vorgelegt von

Oliver Goepfert

aus Hannover

Göttingen 2018 


\section{Betreuungsausschuss:}

Prof. Dr. Andreas Tilgner, Geophysikalische Strömungsmechanik, Institut für Geophysik Göttingen

Prof. Dr. Gert Lube, Numerik partieller Differentialgleichungen, Institut für Numerische und Angewandte Mathematik Göttingen

\section{Mitglieder der Prüfungskommission:}

\section{Referent:}

Prof. Dr. Andreas Tilgner, Geophysikalische Strömungsmechanik, Institut für Geophysik Göttingen

\section{Korreferent:}

Prof. Dr. Gert Lube, Numerik partieller Differentialgleichungen, Institut für Numerische und Angewandte Mathematik Göttingen

\section{Weitere Mitglieder der Prüfungskommission:}

Prof. Dr. Dr. Andreas Dillmann, Institut für Aerodynamik und Strömungstechnik, DLR Göttingen

Prof. Dr. Hans Hofsäss, II. Physikalisches Institut Göttingen

Prof. Dr. Wolfgang Glatzel, Institut für Astrophysik Göttingen

Prof. Dr. Wolfram Kollatschny, Institut für Astrophysik Göttingen

Tag der mündlichen Prüfung: 


\section{Contents}

1 Introduction 1

2 Methods 5

2.1 Mathematical model . . . . . . . . . . . . . . . . . . . . . . 5

2.1 .1 Hydrodynamic Equations of Motion . . . . . . . . . . . . 6

2.1 .2 Induction equation . . . . . . . . . . . . . . . 8

2.2 Computational methods . . . . . . . . . . . . . . . . . . . 9

2.2 .1 Boundary conditions . . . . . . . . . . . . . . . . 9

3 Hydrodynamics $\quad 11$

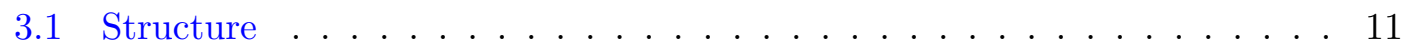

3.1 .1 Kinetic Energy . . . . . . . . . . . . . . . . . . 13

3.2 Radial vorticity distribution . . . . . . . . . . . . . . . . 15

3.3 Single Vortex State . . . . . . . . . . . . . . . . . . . . . . . . 27

3.4 Axisymmetric modes . . . . . . . . . . . . . . . . . . . 35

3.5 Triad resonance . . . . . . . . . . . . . . . . . . . 54

4 Kinematic Dynamo $\quad 69$

4.1 Antisymmetric Magnetic Reynolds Number . . . . . . . . . . . . . . . 71

4.2 Full-Flow Magnetic Reynolds Number . . . . . . . . . . . . . . . . 72

4.3 Rotational Magnetic Reynolds Number . . . . . . . . . . . . . . . . . . . . 75

5 Cuboids $\quad 81$

5.1 Hydrodynamics . . . . . . . . . . . . . . . . . . . . . . . . . . . 81

5.2 Kinematic Dynamo . . . . . . . . . . . . . . . . . . . . . . 84

6 Herzenberg Dynamo $\quad 89$

6.1 Fundamentals . . . . . . . . . . . . . . . . . . . . . . . . . . 89

6.2 Results . . . . . . . . . . . . . . . . . . . . . . 91 
$\begin{array}{lll}7 \text { Conclusion } & 101\end{array}$

7.1 Hydrodynamics . . . . . . . . . . . . . . . . . . . . . . . . . . . 101

7.1 .1 Single Vortex State . . . . . . . . . . . . . . . . . . . . . 101

7.1.2 Axisymmetric modes . . . . . . . . . . . . . . . . . . . 102

7.1.3 Triad resonances . . . . . . . . . . . . . . . . . 103

7.2 Kinematic Dynamo . . . . . . . . . . . . . . . . . . . . . . . . 104

7.3 Herzenberg Dynamo . . . . . . . . . . . . . . . . . . . . . 108

$\begin{array}{ll}\text { Bibliography } & 128\end{array}$ 


\section{List of Figures}

2.1 Basic geometry used in the simulation: A cube with side length $L$ and a coordinate system with axes parallel to the sides of the cube. The rotation axis $\boldsymbol{\omega}_{D}$ is aligned to the z-axis, while the precession axis $\Omega_{P}$ rotates around it with a fixed angle $\alpha$ between them. The origin lies in the center of the cube, around which the rotation takes place. . . . . . . 8

2.2 The graphical representation of free-slip boundary condition at $p_{0}$ with points in bright blue within the fluid volume and points in dark magenta outside of it. $p_{0}$ itself play a special role as the point on the border and $p_{-1}$ and $p_{-2}$ are symmetric halo points which imitate a stress-free flow parallel $(\mathrm{P})$ to the boundary and no flux normal $(\mathrm{N})$ to it. . . . . . . . . 10

3.1 Sketch of a hypothetical experimental setup: The container is mounted on a table, so that it is tilted to $\alpha$. The table rotates with $\boldsymbol{\Omega}_{P}$, while the container itself rotates with $\omega_{D} \ldots \ldots \ldots \ldots \ldots$

3.2 The velocity $u$ in the precession reference frame at $\mathrm{Ek}=2.5 \cdot 10^{-4}, \Omega_{P}=$ -0.04 in a plane spanned by $\boldsymbol{\omega}_{D}$ and $\boldsymbol{\Omega}_{P}$ (left) and as an isosurface at $u=0.05$ (right): In both figures the s-curve caused by the spin-over-mode is clearly visible. . . . . . . . . . . . . . . . . . 13

3.3 kinetic energy $E_{k i n}$ over time $t$ for exemplary precession rates $\Omega_{P}=$ $-0.02 ;-0.05 ;-0.16$ at $E k=2.5 \cdot 10^{-4}$ and $\alpha=60^{\circ}$ : The energy grows fast at the beginning, but saturates around a energy value specific for the precession rate. . . . . . . . . . . . . . . . . . . 14 
3.4 Kinetic energy $E_{k i n}$ for different precession rates $\Omega_{P}$ at Ekman numbers $\mathrm{Ek}=1.0 \cdot 10^{-3}$ (squares); $2.5 \cdot 10^{-4}$ (triangles); $1.0 \cdot 10^{-4}$ (circles); $1.0 \cdot 10^{-5}$ (diamonds): The energy increases with increasing absolute precessing rate smoothly. At Ek $=2.5 \cdot 10^{-4}$ and $1.0 \cdot 10^{-4}$ a strong jump in the energy is seen. A dashed line indicates hysteresis effects: It is obtained by using a starting field from a preceding simulation with a lower absolute value, while the continuous line marks the reverse direction. . . . . . . . . . . . . 14

3.5 Jump in $E_{k i n}$ for a critical precession rate $\Omega_{P}$ : The transition occurs at the marked line . . . . . . . . . . . . . . . . . . . 15

3.6 Definition of the radial distribution: On the left side, the composition of $V_{r}$ is pictured: a spherical shell of radius $r$ and thickness $\Delta r$ wrap around the specific volume for $\boldsymbol{\omega}(r)$. In numeric context all grid points within the shell will be summed up, weighted with the volume they associated with (see sec. 2.2). Of course, with growing $r, V_{r}$ will grow too, but when it starts to intersect with sides of the cube at $r \approx 0.5$, the volume will decrease for bigger $r$, until it does not intersect with the cube anymore at $r \approx 0.5 \sqrt{3} \approx 0.87$ as seen on the right side. This implies that in radial distributions values at $r \approx 0$ and $r>0.6$ will not be as important in mean values over the whole cube volume as they appear in the distribution. . . 16

$3.7 \omega(r)$ (left side) and $\theta(r)$ (right side) for (a) $\mathrm{Ek}=1.0 \cdot 10^{-3}$, (b) $\mathrm{Ek}=$ $2.5 \cdot 10^{-4}$ and (c) $\mathrm{Ek}=1.0 \cdot 10^{-4}$ in the $\left(\Omega_{P}, r\right)$-plane. For points in the white area no data were collected. . . . . . . . . . . . . . . . .

$3.8 \omega(r)$ (left side) and $\theta(r)$ (right side) for (a) $\mathrm{Ek}=2.5 \cdot 10^{-4}$, (b) Ek $=$ $1.0 \cdot 10^{-4}$ and $(\mathrm{c}) \mathrm{Ek}=1.0 \cdot 10^{-5}$ for chosen precession rates $\Omega_{P} \ldots \ldots 18$

$3.9 \omega_{F}\left(\Omega_{P}\right)$ (left) and $\theta_{F}\left(\Omega_{P}\right)$ (right) with $r_{\text {end }}=0.1$ for different Ekman numbers: The transitions at $\mathrm{Ek}=2.5 \cdot 10^{-4}$ and $\mathrm{Ek}=1.0 \cdot 10^{-4}$ can be clearly seen at $\omega_{F}$ while for $\mathrm{Ek}=1.0 \cdot 10^{-3}$ only the first one appears and $\mathrm{Ek}=1.0 \cdot 10^{-5}$ shows none. On $\theta_{F}$ on the other hand, the transitions in $\mathrm{Ek}=2.5 \cdot 10^{-4}$ are notable too but not so for $\mathrm{Ek}=1.0 \cdot 10^{-4}$. At $\Omega_{P}=-0.3 \theta_{F} \approx 1.1$ for all Ekman numbers, a little above $\alpha$, so the rotation axis and the fluid axis are roughly aligned. . . . . . . . . . .

$3.10 \omega_{F}\left(\Omega_{P}\right)$ (left) and $\theta_{F}\left(\Omega_{P}\right)$ (right) with $r_{\text {end }}=0.3$ for different Ekman numbers: the same behavior is shown as in fig. 3.9, but is much more subdued. . . . . . . . . . . . . . . . . . . . . 
3.11 Flow in the planes with $x=0$ (top), $y=0$ (middle) and $z=0$ (bottom) for $\mathrm{Ek}=1.0 \cdot 10^{-3}$ and $\Omega_{P}=-0.05$ : The streamlines represent the velocity projected onto the respective plane while the color scale shows the actual velocity. . . . . . . . . . . . . . . . . . . 22

3.12 Flow (left side) and vorticity (right side) in the planes where $y=0$ for three different precession rates (from top to bottom) $\Omega_{P}=-0.05,-0.12$ and -0.21 at $\mathrm{Ek}=1.0 \cdot 10^{-3}$ corresponding to low, middle and high region. 23

3.13 Streamlines alongside the vorticity $\omega$ at (from top to bottom) $\Omega_{P}=-0.05$, $\Omega_{P}=-0.12$ and $\Omega_{P}=-0.21$ and $\mathrm{Ek}=1.0 \cdot 10^{-3}$ show the growing complexity of the rotation at edges. . . . . . . . . . . . . . . 24

3.14 Flow (left side) and vorticity (right side) in the plane where $y=0$ for three different precession rates (from top to bottom) $\Omega_{P}=-0.05,-0.1$ and -0.2 at $\mathrm{Ek}=2.5 \cdot 10^{-4}$ corresponding to low, middle and high region. 25

3.15 Flow in the plane where $y=0$ for three different precession rates $\Omega_{P}=$ $-0.02,-0.05$ and -0.19 at $\mathrm{Ek}=1.0 \cdot 10^{-4}$ corresponding to low, middle

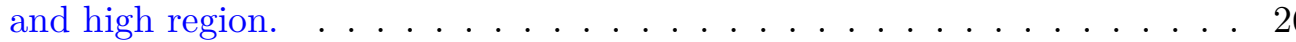

3.16 Flow (left side) and vorticity (right side) in the plane where $y=0$ for three different precession rates (from top to bottom) $\Omega_{P}=-0.02$ and -0.05 at $\mathrm{Ek}=1.0 \cdot 10^{-5}$.

3.17 The reference system for the mantle and for the fluid are sketched here: The mantle reference system $(x, y, z)$ is fixed in respect to the boundaries with the $z$-axis alongside the rotation axis, the fluid reference system $\left(x^{\prime}, y^{\prime}, z^{\prime}\right)$ moves inside the boundaries, so that $z^{\prime}$ points alongside $\boldsymbol{\omega}_{F} \ldots 28$

$3.18 \omega_{z^{\prime}}$ for $\mathrm{Ek}=1.0 \cdot 10^{-5}$ and $\Omega_{P}=-0.02$ in the plane normal (left) and parallel (right) to $z^{\prime}$ through the origin. . . . . . . . . . . . . . 29

$3.19 \omega_{z^{\prime}}$ for $\mathrm{Ek}=1.0 \cdot 10^{-5}$ and $\Omega_{P}=-0.04$ in the plane normal (left) and parallel (right) to $\boldsymbol{z}^{\prime}$ through the origin. . . . . . . . . . . . . 29

$3.20 \omega_{z^{\prime}}$ (right) for $\mathrm{Ek}=1.0 \cdot 10^{-4}$ and $\Omega_{P}=-0.1$ in the plane normal (left) and parallel (right) to $z^{\prime}$ through the origin. . . . . . . . . . . . . 29

3.21 The parameters $R_{C l}$ and $r_{F_{C l}}$ illustrated: If a cluster (grey area) is reformed to a perfect circle (dashed line), $R_{C l}$ defines the radius of this circle, while $r_{F_{C l}}$ is the distance between its center and the origin. . . . . 30 
$3.22 R_{C l}\left(r_{F_{C l}}\right)$ (left side) and clusters on $z=0$-plane through the origin at a random point of time (right side) for (from top to bottom) $\mathrm{Ek}=1.0 \cdot 10^{-5}$ and $\Omega_{P}=-0.02, \mathrm{Ek}=1.0 \cdot 10^{-5}$ and $\Omega_{P}=-0.03$ and $\mathrm{Ek}=1.0 \cdot 10^{-5}$

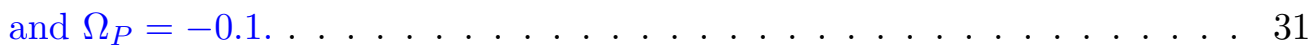

$3.23 R_{C l}\left(r_{F_{C l}}\right)$ (left side) and clusters on $z=0$-plane through the origin at a random point of time (right side) for $\mathrm{Ek}=2.5 \cdot 10^{-4}$ and $\Omega_{P}=-0.1$ (top), $\mathrm{Ek}=3.0 \cdot 10^{-4}$ and $\Omega_{P}=-0.13$ (bottom) . . . . . . . .

$3.24 R_{C l}\left(r_{F_{C l}}\right)$ (top, left side) and clusters on $z=0$-plane through the origin at a random point of time (top, right side) for $\mathrm{Ek}=1.0 \cdot 10^{-4}$ and $\Omega_{P}=-0.03$ $R_{C l}\left(\tilde{r}_{F_{C l}}\right)$ (bottom) for the same parameters. . . . . . . . . . . 34

$3.25 R_{\text {vortex }}$ for $\mathrm{Ek}=2.5 \cdot 10^{-4}$ (upward triangles), $\mathrm{Ek}=1.0 \cdot 10^{-4}$ (circles), $\mathrm{Ek}=5.0 \cdot 10^{-5}$ (diamonds) and $\mathrm{Ek}=1.0 \cdot 10^{-5}$ (downward triangles) $\ldots 36$

3.26 Position of the center of the central vortex at $\mathrm{Ek}=1.0 \cdot 10^{-5}$ and $\Omega_{P}=$ -0.02 for 16 rotations. . . . . . . . . . . . . . . . . . 36

3.27 Time dependent variation of $R_{\text {vortex }}(t)$ for $\mathrm{Ek}=3.0 \cdot 10^{-4} \Omega_{P}=-0.13$ (top), $\mathrm{Ek}=5.0 \cdot 10^{-5} \Omega_{P}=-0.04$ (middle) and $\mathrm{Ek}=1.0 \cdot 10^{-5} \Omega_{P}=$ -0.02 (bottom): The regular appearance of $\omega>0$ vortices at $\mathrm{Ek}=$ $3.0 \cdot 10^{-4}$ becomes chaotic at lower Ekman numbers but are maintained for a longer time until at $\mathrm{Ek}=1.0 \cdot 10^{-5}$ they stay permanent at specific precession rates as $\Omega_{P}=-0.02 \ldots \ldots \ldots \ldots \ldots$

3.28 Relative time $\frac{t_{\text {vortex }}}{T}$ the vortex fulfills $R_{\text {vortex }}>0.01$ for $\mathrm{Ek}=2.5 \cdot 10^{-4}$ (upward triangles), $\mathrm{Ek}=1.0 \cdot 10^{-4}$ (circles), $\mathrm{Ek}=5.0 \cdot 10^{-5}$ (diamonds) and $\mathrm{Ek}=1.0 \cdot 10^{-5}$ (downward triangles) $\ldots \ldots \ldots 38$

3.29 Median of vortex lifespan $\tau_{\text {vortex }}$ under condition $R_{\text {vortex }}(t)>0.01$ for $\mathrm{Ek}=2.5 \cdot 10^{-4}$ (upward triangles), $\mathrm{Ek}=1.0 \cdot 10^{-4}$ (circles), $\mathrm{Ek}=5.0 \cdot 10^{-5}$ (diamonds) and $\mathrm{Ek}=1.0 \cdot 10^{-5}$ (downward triangles) does extend radically for $\mathrm{Ek}<10^{-4}$. At $\Omega_{P}=-0.02$ and $\mathrm{Ek}=1.0 \cdot 10^{-5}$ the condition is always satisfied, so $\tau_{\text {vortex }} \rightarrow \infty \ldots \ldots \ldots \ldots \ldots \ldots$

3.30 Identified clusters for the whole equatorial plane for $\mathrm{Ek}=1.0 \cdot 10^{-5}$ and $\Omega_{P}=-0.02$ at $t=100.0$ (left) and $t=203.6$ (right) show the degradation of the single vortex into smaller clusters. . . . . . . . . . . . . . .

3.31 Number of clusters at one time step identified with $\omega_{z}(r<0.2)>0$ for $\mathrm{Ek}=1.0 \cdot 10^{-5}$ and $\Omega_{P}=-0.02$ often is equal or less than three, only to exceed 10 clusters at one point of time at $t \approx 60$ and $200 . \ldots \ldots$. . . 
3.32 Map of all runs made for investigating vortex state: dots mark runs with $R_{\text {vortex }}>0.01$, crosses the remaining runs. . . . . . . . . . . . . 40

3.33 The cubic boundaries enforce a mode on the flow in the components $u_{r}(z=0)$ and $u_{\phi}(z=0)$ (here in the mean field at $\mathrm{Ek}=2.5 \cdot 10^{-4} ; \Omega_{P}=$ $-0.16)$, while the $u_{z}(z=0)$-component is dominated by the $m=1$-spinover-mode enforced by the precession. . . . . . . . . . . . . . . . .

3.34 For $\mathrm{Ek}=2.5 \cdot 10^{-4}, \Omega_{P}=-0.16 u_{z}(r, z)$ (top) and $u_{r}(r, z)$ (middle) exhibits a clear visible mode in $z$ and $r$ symmetric to the plane $z=0$, while $u_{\phi}(r, z)$ is dominated by a negative motion homogeneous in $z$-direction. 41

3.35 The flow in the $r$ - $z$-plane at $\mathrm{Ek}=2.5 \cdot 10^{-4}, \Omega_{P}=-0.16$ forms to a double roll with counterrotating rolls. . . . . . . . . . . . . . . . . .

3.36 Contour plots for $u_{z}(z)+u_{z}(-z)$ in planes with $z=0.3$ and $y=0.0$ at $\mathrm{Ek}=2.5 \cdot 10^{-4}$ and $\Omega_{P}=-0.16$ show the actual composition which lead to the form of the $m=0$ if taken the radial mean value. . . . . . . . . 43

3.37 Non-zero amounts of $E_{m=0, z} / E_{m \neq 0, z}$ (continuous line) exist for $\mathrm{Ek}=2.5$. $10^{-4}, \mathrm{Ek}=1.0 \cdot 10^{-4}$ only in the same region of $\Omega_{P}$ like the middle region presented in sec. 3.1. For $\mathrm{Ek}=1.0 \cdot 10^{-5}$ the amount is much smaller than by higher Ekman numbers, except for the peak by $\Omega_{P}=-0.03$, which also occurs at $\mathrm{Ek}=1.0 \cdot 10^{-4}, \Omega_{P}=-0.04$. Considering only the x-y-plane-symmetric energy $E_{m=0, z, s} / E_{m \neq 0, z}$ (dashed line) which leads to the double roll described in 3.35, the peak is not seen. For Ek $=1.0 \cdot 10^{-3}$ $E_{m=0, z} / E_{m \neq 0, z}$ seems to correlate with the middle region, too, but shows other behavior in antisymmetric amounts and also in non-zero amounts below the lower limit. . . . . . . . . . . . . . . . . . . . . . 44

3.38 Time evolution of $E_{m=0, \phi}$ and $\left(E_{m=0, z}+E_{m=0, r}\right)$ at $\mathrm{Ek}=2.5 \cdot 10^{-4}$ for $\Omega_{P}=-0.16,-0.11,-0.09$ with a dashed line indicating the symmetric parts of $\left(E_{m=0, z}+E_{m=0, r}\right)$ : every precession rate shows a different behavior in energy of axisymmetric modes, stable, a breakdown to chaotic behavior with recognizable antisymmetric amount and oscillating. . . . . .

$3.39 \boldsymbol{u}_{p o l}, \boldsymbol{u}_{p o l, s}$ and $\boldsymbol{u}_{p o l, a}$ (from top to bottom) at Ek $=2.5 \cdot 10^{-4}, \Omega_{P}=-0.11$ at time points $t=89.9$ (left side) and $t=250.1$ (right side) . . . . . 46

3.40 Angular frequency of axisymmetric oscillations $\omega_{m=0}$ at $\mathrm{Ek}=3.0 \cdot 10^{-4}$ (squares), $\mathrm{Ek}=2.5 \cdot 10^{-4}$ (triangles) and $\mathrm{Ek}=1.0 \cdot 10^{-4}$ (circles) for different $\Omega_{P}$ is constant for variation of Ekman number but increases with increasing precession rate. . . . . . . . . . . . . . . . . 47 
3.41 Time evolution of $E_{m=0, \phi}$ and $\left(E_{m=0, z}+E_{m=0, r}\right)$ at $\mathrm{Ek}=1.0 \cdot 10^{-4}$ (top) $\Omega_{P}=-0.08$ (left side) and $\Omega_{P}=-0.04$ (right side) and Ek $=1.0 \cdot 10^{-4}$ (bottom) $\Omega_{P}=-0.05$ (left side) and $\Omega_{P}=-0.03$ (right side) with a dashed line indicating the symmetric parts of $\left(E_{m=0, z}+E_{m=0, r}\right)$ : The behavior of axisymmetric energy becomes more chaotic at lower Ekman numbers and breakdowns appear more often. . . . . . . . . . . . . 48

$3.42 \boldsymbol{u}_{\text {pol }}$ at $\mathrm{Ek}=1.0 \cdot 10^{-5}$ for $\Omega_{P}=-0.05$ (left side) and $\Omega_{P}=-0.03$ (right side): At $\Omega_{P}=-0.05$ the axisymmetric flow mainly consists of the double roll, while at $\Omega_{P}=-0.3$ the antisymmetric single roll predominant is in the flow. . . . . . . . . . . . . . . . . . . . . . 49

3.43 At lower precession rates $\Omega_{P} \leq-0.18$ the antisymmetric component vanishes for $\mathrm{Ek}=1.0 \cdot 10^{-3}$ and only the double roll as seen here for $\Omega_{P}=-0.3$ persist. . . . . . . . . . . . . . . . . . 50

3.44 Map of regions with axisymmetric modes and single vortex state: Blue area defines occurrence of axisymmetric modes with $E_{m=0, z} / E_{m \neq 0, z}>0.1$ and orange areas show the single vortex state with $R_{\text {vortex }}>0.01$. . . . 5 51

3.45 Comparison of time evolution of $E_{m=0, r}+E_{m=0, z}$ and $R_{\text {vortex }}$ for different parameters: Oscillations in $E_{m=0, r}+E_{m=0, z}$ occur parallel to oscillations in $R_{\text {vortex }}$ with the same frequency, at breakdowns, both become chaotic, but with no notable correlation. . . . . . . . . . . . . . . . . 5

3.46 The different phases of axisymmetric modes for different $\Omega_{P}$ from -0.2 to -0.01 and $\mathrm{Ek}$ from $5.0 \cdot 10^{-5}$ to $3.0 \cdot 10^{-4}$ : Time independent stable (squares), oscillating (circles), chaotic breakdown of (triangles) and non-existent modes (crosses). The single vortex regime of sec. 3.3 is highlighted in grey. . . . . . . . . . . . . . . . . . . . 5

3.47 The main two modes in $m=0$ occurring are sketched here: $m=0 k=1$ (here known as $\left.s_{1} t_{1}\right)$ on the right side, $m=0 k=2\left(s_{2} t_{1}\right)$ on the left side. 54

$3.48 E_{m=0, \phi}$ for $\mathrm{Ek}=1.0 \cdot 10^{-3}$ (squares), $\mathrm{Ek}=2.5 \cdot 10^{-4}$ (triangles), $\mathrm{Ek}=1.0 \cdot 10^{-4}$ (circles), $\mathrm{Ek}=1.0 \cdot 10^{-5}$ (diamonds) is the main contribution to total kinetic energy $E_{k i n}$ (see fig. 3.4) . . . . . . . . . . . . . . . 55 
3.49 The relation $\Gamma_{0}=\left(E_{m=0, r}+E_{m=0, z}\right) / E_{m=0, \phi}$ is qualitatively similar to fig. 3.37, but decreases overall with decreasing Ekman number after achieving its highest value at $\mathrm{Ek}=2.5 \cdot 10-4$. The dashed line shows only the symmetric part $E_{m=0, s, r}+E_{m=0, s, z} / E_{m=0, \phi}$, while the continuous line shows the total value of $E_{m=0, r}+E_{m=0, z} / E_{m=0, \phi}$. Differences between the two indicate existence of a $s_{1} t_{1}$-mode. . . . . . . . . . . . . . 56

3.50 Curves of $u_{m=0, z}(r)$ for roughly 3000 points in time over 50 rotations (grey lines) and their mean value (black line) for free-slip (left side) and no-slip (right side) boundary conditions at $\mathrm{r}=0.45$ : For both shown parameter combinations, $\mathrm{Ek}=2.5 \cdot 10^{-4}, \Omega_{P}=-0.12(\mathrm{top})$ and $\mathrm{Ek}=1.0 \cdot 10^{-4}, \Omega_{P}=$ -0.1 (bottom), the no-slip cases show less variation from the mean value, which shows the form matching the double roll. . . . . . . . . . . . . . 57

$3.51 E_{a}\left(\Omega_{P}\right)$ behaves different for the varying Ekman numbers: Ek $=1.0 \cdot 10^{-3}$ (squares) only has a sharp defined area with $E_{a} \neq 0$, while at Ek $=$ $2.5 \cdot 10^{-4}$ (triangles) two different regions can be identified. $\mathrm{Ek}=1.0 \cdot 10^{-4}$ (circles) and $\mathrm{Ek}=1.0 \cdot 10^{-5}$ (diamonds) have antisymmetric components over the whole examined region except at very small precession rates. Also they peak each for $\left|\Omega_{P}\right| \leq 0.03$. The different line styles indicates hysteresis effects: The dashed lines originate from changing $\Omega_{P}$ to zero from left to right in the figure while continuous line is obtained from the opposite direction. . . . . . . . . . . . . . . . . 5 58

3.52 sketch of unstable modes coupled in a triad resonance by viscous instabilities from [Lorenzani, 2002]. . . . . . . . . . . . . . . . 59

$3.53 u_{a, z^{\prime}}$ (left) and $u_{a, r^{\prime}}$ (right) for Ek $=2.5 \cdot 10^{-4}$ and $\Omega_{P}=-0.02$ in the plane normal to $\boldsymbol{z}^{\prime}$ through the origin. A azimuthal 4-mode in $u_{a, z^{\prime}}$ and a 5 -mode in $u_{a, r^{\prime}}$ is visible. . . . . . . . . . . . . . 60

$3.54 \omega_{a, z^{\prime}}$ for $\mathrm{Ek}=2.5 \cdot 10^{-4}$ and $\Omega_{P}=-0.02$ in the plane normal to $\boldsymbol{z}^{\prime}$ through

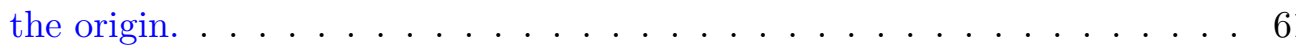

$3.55 u_{a, z^{\prime}}$ (left) and $u_{a, r^{\prime}}$ (right) for $\mathrm{Ek}=2.5 \cdot 10^{-4}$ and $\Omega_{P}=-0.02$ on a cylindrical surface along $\boldsymbol{z}^{\prime}$ and radius $R=0.3$. The same modes as in fig. 3.53 are visible in azimuthal direction, but in $z^{\prime}$-direction the antisymmetric flow grows much more complex because of the constraints by the boundary. . . . . . . . . . . . . . . . . . . 6 62 
$3.56 u_{a, z^{\prime}}$ (left) and $\omega_{a, z^{\prime}}$ (right) for $\mathrm{Ek}=2.5 \cdot 10^{-4}$ and $\Omega_{P}=-0.08$ in the plane normal to $\boldsymbol{z}^{\prime}$ through the origin. A azimuthal 2-mode in $u_{a, z^{\prime}}$ and a 1 -mode in $\omega_{a, z^{\prime}}$ is visible. . . . . . . . . . . . . . . . .

$3.57 u_{a, z^{\prime}}$ (left) and $\omega_{a, z^{\prime}}$ (right) for $\mathrm{Ek}=2.5 \cdot 10^{-4}$ and $\Omega_{P}=-0.16$ in the plane normal to $\boldsymbol{z}^{\prime}$ through the origin. A azimuthal 2-mode in $u_{a, z^{\prime}}$ and a 1 -mode in $\omega_{a, z^{\prime}}$ is visible. . . . . . . . . . . . . . . . . 64

$3.58 E_{a}(t)$ at $\mathrm{Ek}=2.5 \cdot 10^{-4}$ for the first unstable flow around $\Omega_{P}=-0.02$ oscillates, while at the second occurrence of antisymmetric energy at lower precession rates $E_{a}(t)$ is small at the unset like at $\Omega_{P}=-0.05$ and quickly gains a chaotic time dependence when increasing $\left|\Omega_{P}\right| \ldots \ldots \ldots$. . . .

$3.59 E_{a}(t)$ at $\mathrm{Ek}=2.5 \cdot 10^{-4}$ at $\Omega_{P}<-0.08$ remains chaotic in nature, with the special case around $\Omega_{P} \approx-0.16$ which, while chaotic, shows much less variance. . . . . . . . . . . . . . . . . . . . . . .

$3.60 u_{a, z^{\prime}}$ (left) and $\omega_{a, z^{\prime}}$ (right) for Ek $=1.0 \cdot 10^{-4}$ and $\Omega_{P}=-0.008$ in the plane normal to $\boldsymbol{z}^{\prime}$ through the origin. A azimuthal 2-mode in $u_{a, z^{\prime}}$ and a 1 -mode in $\omega_{a, z^{\prime}}$ is visible. . . . . . . . . . . . . . .

$3.61 u_{a, z^{\prime}}$ (left) and $\omega_{a, z^{\prime}}$ (right) for $\mathrm{Ek}=1.0 \cdot 10^{-4}$ and $\Omega_{P}=-0.008$ in the plane normal to $\boldsymbol{z}^{\prime}$ through the origin. A azimuthal 2-mode in $u_{a, z^{\prime}}$ and a 1 -mode in $\omega_{a, z^{\prime}}$ is visible. . . . . . . . . . . . . . . 66

$3.62 E_{a}(t)$ at $\mathrm{Ek}=1.0 \cdot 10^{-4}$ devolves even faster into chaotic behavior than at $\mathrm{Ek}=2.5 \cdot 10^{-4}$ in fig. 3.58 and $3.59 \ldots \ldots \ldots 66$

$3.63 u_{a, z^{\prime}}$ (left) and $\omega_{a, z^{\prime}}$ (right) for $\mathrm{Ek}=1.0 \cdot 10^{-4}$ and $\Omega_{P}=-0.03$ in the plane normal to $z^{\prime}$ through the origin. . . . . . . . . . . . . . 66

$3.64 u_{a, z^{\prime}}$ (left) and $\omega_{a, z^{\prime}}$ (right) for $\mathrm{Ek}=1.0 \cdot 10^{-4}$ and $\Omega_{P}=-0.04$ in the plane normal to $z^{\prime}$ through the origin. . . . . . . . . . . . . . .

3.65 Power density spectrum of characteristic velocity components $u_{a, r}, u_{a, z}$, $u_{s, r}$ and $u_{s, z}$ at $\mathrm{Ek}=1.0 \cdot 10^{-4}$ and $\Omega_{P}=-0.008$ for the stable hysteresis branch with $E_{a}=0$ (left) and for the unstable branch with $E_{a} \neq 0$ (right), normalized to the peak of the spin-over mode. . . . . . . . . . . . .

3.66 Power density spectrum of characteristic velocity components $u_{a, r}, u_{a, z}$, $u_{s, r}$ and $u_{s, z}$ at $\mathrm{Ek}=1.0 \cdot 10^{-4}$ and $\Omega_{P}=-0.035$ (left) and at $\mathrm{Ek}=$ $2.5 \cdot 10^{-4}$ and $\Omega_{P}=-0.02$ (right), normalized to the peak of the spin-over mode. 
3.67 Power density spectrum of characteristic velocity components $u_{a, r}, u_{a, z}$, $u_{s, r}$ and $u_{s, z}$ at Ek $=2.5 \cdot 10^{-5}$ and $\Omega_{P}=-0.05$ (left) and $\Omega_{P}=$ -0.08 (right), normalized to the peak of the spin-over mode. . . . . . . 68

4.1 Time evolution of magnetic energy $E_{B}(t)$ (left) and growth factor $p$ (right) for different $\mathrm{Pm}$ at $\mathrm{Ek}=2.5 \cdot 10^{-4}$ and $\Omega_{P}=-0.16 \ldots 70$

$4.2 \operatorname{Pm}_{c}(\mathrm{Ek})$ at $\Omega_{P}=-0.02$ (squares), $\Omega_{P}=-0.05$ (upward triangles), $\Omega_{P}=$ -0.1 (circles), $\Omega_{P}=-0.16$ (diamonds), $\Omega_{P}=-0.3$ (downward triangles) decreases with decreasing Ek. . . . . . . . . . . . . 71

$4.3 \mathrm{Rm}_{\mathrm{c}, \mathrm{a}}$ for $\mathrm{Ek}=1.0 \cdot 10^{-4}($ circles $)$ and $\mathrm{Ek}=2.5 \cdot 10^{-4}$ (triangles): For $\mathrm{Ek}=1.0 \cdot 10^{-4}$ and $\Omega_{P}>=-0.02$ hydrodynamic simulations of the unstable branch were taken. . . . . . . . . . . . . . . . 73

$4.4 \mathrm{Rm}_{\mathrm{c}, \mathrm{kin}}$ for $\mathrm{Ek}=1.0 \cdot 10^{-3}$ (squares), $\mathrm{Ek}=2.5 \cdot 10^{-4}$ (triangles), $\mathrm{Ek}=$ $1.0 \cdot 10^{-4}$ (circles) and $\mathrm{Ek}=1.0 \cdot 10^{-5}$ (diamonds). . . . . . . . . 74

4.5 Vorticity $\omega$ (left) and magnetic field $B$ (right) at $\mathrm{Ek}=1.0 \cdot 10^{-3}$ and $\Omega_{P}=-0.25$ with $\mathrm{Pm}=10 \ldots \ldots \ldots \ldots \ldots$

4.6 Vorticity $\omega$ (left) and magnetic field $B$ (right) at $\mathrm{Ek}=1.0 \cdot 10^{-5}$ and $\Omega_{P}=-0.02$ with $\mathrm{Pm}=0.3 \ldots \ldots \ldots \ldots \ldots$

$4.7 \mathrm{Rm}_{\mathrm{c}, \mathrm{rot}}$ for $\mathrm{Ek}=1.0 \cdot 10^{-3}$ (squares), $\mathrm{Ek}=2.5 \cdot 10^{-4}$ (triangles), $\mathrm{Ek}=$ $1.0 \cdot 10^{-4}$ (circles) and $\mathrm{Ek}=1.0 \cdot 10^{-5}$ (diamonds) . . . . . . . . 76

$4.8 \mathrm{Rm}_{\mathrm{c}, \text { rot }}$ for $\Omega_{P}=-0.02$ (squares), $\Omega_{P}=-0.05$ (upward triangles), $\Omega_{P}=$ -0.1 (circles), $\Omega_{P}=-0.16$ (diamonds) and $\Omega_{P}=-0.3$ (downward triangles) 77

$4.9 L_{D}$ against $\mathrm{Ek}$ for $\Omega_{P}=-0.02$ (squares), $\Omega_{P}=-0.05$ (upward triangles), $\Omega_{P}=-0.1$ (circles), $\Omega_{P}=-0.16$ (diamonds) and $\Omega_{P}=-0.3$ (downward

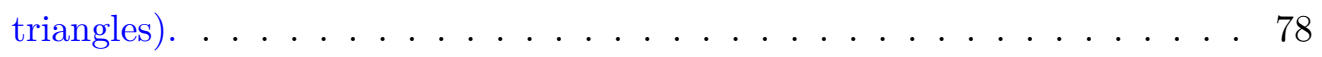

$4.10 \mathrm{Rm}_{\mathrm{c}, \mathrm{rot}}$ against $L_{D}$ for $\Omega_{P}=-0.02$ (squares), $\Omega_{P}=-0.05$ (upward triangles), $\Omega_{P}=-0.1$ (circles), $\Omega_{P}=-0.16$ (diamonds) and $\Omega_{P}=-0.3$ (downward triangles).

$5.1 E_{k i n}$ at $\mathrm{Ek}=2.5 \cdot 10^{-4}$ for $\eta=-0.7$ (squares), $\eta=-0.5$ (triangles),

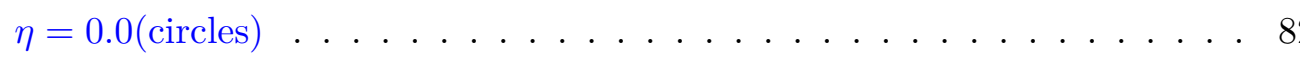

$5.2 E_{a}$ at $\mathrm{Ek}=2.5 \cdot 10^{-4}$ for $\eta=-0.7$ (squares), $\eta=-0.5$ (triangles), $\eta=$ 0.0 (circles) . . . . . . . . . . . . . . . . . 82

$5.3 \frac{E_{a}}{E_{k i n}}$ at $\mathrm{Ek}=2.5 \cdot 10^{-4}$ for $\eta=-0.7$ (squares), $\eta=-0.5$ (triangles),

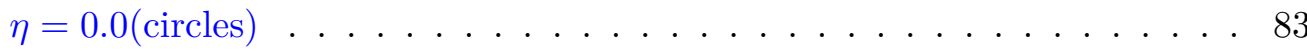


$5.4 u_{a, z^{\prime}}$ (left) and $\omega_{a, r^{\prime}}$ (right) for $\eta=-0.7$ at Ek $=2.5 \cdot 10^{-4}$ and $\Omega_{P}=-0.1$ in the plane normal to $z^{\prime}$ through the origin. . . . . . . . . . . 83

$5.5 \frac{E_{a}}{E_{k i n}}$ at $\Omega_{P}=-0.1$ for $\eta=-0.7$ (squares), $\eta=0.0$ (circles) $\ldots \ldots \ldots 84$

$5.6 E_{k i n}$ at $\Omega_{P}=-0.1$ for $\eta=-0.7$ (squares), $\eta=0.0$ (circles) $\ldots \ldots . . .85$

$5.7 \operatorname{Rm}_{c, k i n}$ at $\Omega_{P}=-0.1$ for $\eta=-0.7$ (squares), $\eta=0.0$ (circles) $\ldots \ldots . \quad 86$

$5.8 \mathrm{Rm}_{c, \text { rot }}$ at $\Omega_{P}=-0.1$ for $\eta=-0.7$ (squares), $\eta=0.0$ (circles) . . . . 86

$5.9 L_{D}$ at $\Omega_{P}=-0.1$ for $\eta=-0.7$ (squares), $\eta=0.0$ (circles) . . . . . . . 87

6.1 The model as it was used by [Brandenburg et al., 1998]: Two spheres of radius $a$ and at a distance $2 d$ of each other rotate around axes, which are tilted inside the $x$-y-planes by an angle $2 \phi$ against each other. The electromagnetic properties of the spheres and their surrounding are the same. The confining bounding box necessary for a simulation is not visualized. . 90

6.2 The original cuboid model is modified by implementing a solid phase which divides the inner space into two smaller cuboids. The solid phase shows the same electromagnetic properties like the remaining fluid phases, but got itself zero velocity in the mantle system. The boundaries facing the fluid phases use the same free-slip conditions like the outer boundaries. 91

6.3 For first test runs the solid barrier is extended to the lower boundary of the whole container, so that cuboid $C_{2}$ vanishes and the field in $C_{2}$ only interacts with the solid phase. . . . . . . . . . . . . . . .

6.4 The velocity profile at $\mathrm{Ek}=10^{-4} ; \Omega_{P}=-0.1 ; \mathrm{Pm}=2$ in a container of form $\left(L_{x}, L_{y}, L_{z}\right)=(1,1,2.1)$ but with $L_{s}=1.1, L_{1}=1$ and $L_{2}=0$ shows the transition between solid and fluid phase at $(x, y)=(-0.25,-0.25)$. The no-flux boundary condition in $z$-direction provide for a continuous curve in $u_{z}$ at the transition $z=1.1$ (dashed vertical line), but $u_{x}$ and $u_{y}$ become discontinuous because of the stress-free condition. . . . . . . . . . 93

6.5 The magnetic components for the same parameters as in fig. 6.4 vanishes in the solid phase except for a small peak at $z=1.1 \ldots \ldots$. . . . . . .

6.6 The peak in magnetic components at $z=1.1$ shown in a smaller cut-out of fig. 6.5 is more specifically in components $B_{x}$ and $B_{y}$ while in $B_{z}$ no peak is seen. . . . . . . . . . . . . .

6.7 The normalized magnetic energy decays exponentially in the solid phase and develops a very weak mode. At the $z=1$.1-border a small peak exists, likely generated by the discrete transition between the two states. . 96 
6.8 The Herzenberg setup at $\mathrm{Ek}=5 \cdot 10^{-4}, \Omega_{P}=-0.04$ generates two identical flows in the mantle velocity $\boldsymbol{u}_{\text {mantle }}($ left side) separated by the notable solid barrier between them as seen here in a cut through the center of the total container alongside the $z$ - $x$-plane. The magnetic field $\boldsymbol{B}$ (right side) at $\mathrm{Pm}=6$, likely produced by shearing effects on the sides, can be seen propagating into the solid phase from both sides. . . . . . . . . . . . 97

6.9 Similar to fig. 6.8 the mantle velocity $\boldsymbol{u}_{\text {mantle }}$ (left side) at Ek $=5 \cdot 10^{-4}$, $\Omega_{P}=-0.35$ is identical in both containers, as is the magnetic field $\boldsymbol{B}$ (right side), here at $\mathrm{Pm}=7$, even though it seems to be more complicated. . . . 97

6.10 The normalized magnetic energy $\hat{\epsilon}_{B}(z)$ for the $(1,0.1,1)$-setup (continuous line) at $\Omega_{P}=-0.04, \mathrm{Ek}=5 \cdot 10^{-4}$ is at maximum amidst the containers while it distributes more homogeneous along the $z$-axis in a cube (dashed line) at same parameters. At the solid barrier, the Herzenberg setup shows the peak in the energy typical for the boundary transition. . . . . . . . . 98

6.11 The normalized magnetic energy $\hat{\epsilon}_{B}(z)$ for the $(1,0.1,1)_{H}$-setup (continuous line) at $\Omega_{P}=-0.35, \mathrm{Ek}=5 \cdot 10^{-4}$ decreases from the outer borders of $z$ to the middle of the whole container. The matching cube (dashed line) has a much more even distribution. . . . . . . . . . . . . . 98

6.12 The normalized magnetic energy $\hat{\epsilon}_{B}(z)$ for the $(0.5,0.1,1.5)_{H}$-setup (continuous line) and the $(0.66,0.1,1.34)_{H}$-setup (dashed line) shows the concentration of the magnetic energy inside one of the containers which is most likely produced by the flow inside the corresponding container alone, while in the opposite container only a small remaining magnetic field exists. 99

6.13 The axial angle $\theta_{F}$ of the fluid axis does not differ greatly between cube and cuboid with $\eta=-0.7$ for $\Omega_{P}<-0.08 \ldots \ldots$. . . . . . . . . 99

6.14 The azimuthal angle $\varphi_{F}$ for cube and cuboid with $\eta=-0.7$ get two distinct nontrivial values, in the regions $\Omega_{P} \in[-0.18:-0.14]$ and $\Omega_{P} \in$

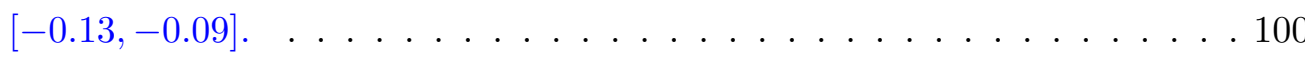

7.1 $\mathrm{Rm}_{\mathrm{c}, \text { rot }}\left(\Gamma_{0}\right)$ for the different phases of axisymmetric modes taken from parameter pairs of $\Omega_{P}$ from -0.2 up to -0.01 and Ek from $5.0 \cdot 10^{-5}$ to $3.0 \cdot 10^{-4}$ : time independent stable (squares), oscillating (circles), chaotic breakdown of (triangles) and non-existent modes (crosses). . . . . . . 106 


\section{List of Tables}

3.1 Mean values of $E_{m=0, z}, \Gamma_{0}$ and $E_{m=0, z} / E_{m \neq 0, z}$ over 50 rotations for three different parameter combinations with no-slip boundary conditions and the equivalent data with free-slip boundary conditions. . . . . . . . . . . . 54

3.2 frequencies $\omega$ for triads at $\mathrm{Ek}=1.0 \cdot 10^{-4} \ldots \ldots \ldots \ldots$. . . . . . 62

4.1 Prominent sets of values of different definitions of magnetic Reynolds number. . . . . . . . . . . . . . . . . . . 79

5.1 The used values of $\eta$ and the translation into edge lengths of cuboids. . . 81

5.2 Selected values of $\mathrm{Rm}_{c, \text { kin }}$ and $\mathrm{Rm}_{c, \text { rot }}$ at $\Omega_{P}=-0.1$ for cuboid with $\eta=-0.7$ and the cube $(\eta=0.0)$ for comparison. . . . . . . . 86

6.1 Resulting critical magnetic Prandtl numbers $\mathrm{Pm}_{c}$ for a range of different containers and two different precession rates $\Omega_{P}=-0.04$ and $\Omega_{P}=$ -0.35 , but all at $\mathrm{Ek}=5 \cdot 10^{-4}$. The first three container used a solid barrier to simulate Herzenberg dynamos while the last three were simple cuboids with accordingly chosen $L_{z}$ for comparison purpose. . . . . . . . . 94

6.2 The critical magnetic Prandtl numbers $\mathrm{Pm}_{c}$ for the $(1,0.1,1.8257)_{H}$-setup and the adequate stand-alone containers are all at the same level between 3.9 and 5 with the exception of the very low $\mathrm{Pm}_{c}=1.96$ for a cube at $\Omega_{P}=-0.18$. The Herzenberg setup never manages to undercut the critical numbers of the sole containers. . . . . . . . . . . . . . . 96 


\section{Introduction}

The idea, that the magnetic field surrounding Earth is generated by motion of conductive fluids inside the core, exists for quite some time. Nevertheless the exact mechanisms inducing the field are still discussed. [Roberts, 1972] showed that a two-dimensional flow is able to sustain a magnetic field while the Ponomarenko dynamo[Ponomarenko, 1973] proved the same for a helical motion within cylindrical boundaries. A lot of possible physical causes were found and examined like convection [Braginsky and Roberts, 1995], tidal forcing [Cébron and Hollerbach, 2014], libration [Wu and Roberts, 2013] and precession [Tilgner, 2005]. Precession is a viable mechanism, as was found out by [Bullard, 1949] on purely energetic grounds but with the caveat, that the known stable solution established by [Poincaré, 1910] for precessing elliptical spheroids can not generate the differential motion necessary for magnetic action. At this point, it becomes clear, that if precession shall generate a reasonable magnetic field, it has to depend on instabilities disturbing the Poincaré flow. The experiments by [Malkus, 1968] established, that the precessing flow can easily reach a catastrophic breakdown and is actually unstable for a sufficient rate of precession. This is supported by the work of [Vanyo et al., 1995] for a spheroid modelled as closely to earth as possible reproducing the results for this experiment. Similarly, [Manasseh, 1992] described more precisely the modes developing in a cylindrical container through instabilities while breaking down. The nature of the underlying instabilities is subject of research until now. [Malkus, 1968] theorized, that axisymmetric shear layers within the fluid become unstable and therefore only nonideal fluids are prone to these instabilities. Differing from this aspect, [Kerswell, 1993] suggested the existence of inertial instabilities in spheroids even in nonviscous fluid. The strain on the flow through the ellipticity of the container excites two resonant inertial modes, known as triadic resonance. The difference for spheroid containers between inertial and viscous instabilities is well documented by the numerical simulations of [Lorenzani and Tilgner, 2001, Lorenzani and Tilgner, 2003]: To distinguish between viscous instabilities caused by the shear with the boundaries from inertial instabilities they used stress-free boundary condition instead of the no-slip condition more relevant 
to experiments. Of course, fluids in experimental setups are best described by no-slip conditions, but numerical simulations offer the opportunity to reduce the influences into a system to analyze cases which would not be possible in realistic experiments. Later, [Lagrange et al., 2008] found that the triad resonance also could be generated by the forced inertial mode inside a precessing system, showing this in an experimental setup by [Lagrange et al., 2011] similar to the setup used by [Manasseh, 1992]. This introduces an additional inertial instability which is not bound to ellipsoid container geometries. Naturally, if one would try to repeat the free-slip boundary simulations of [Lorenzani and Tilgner, 2003] with an axisymmetric container and no ellipticity, nothing would drive the flow to actually excite instabilities. This calls for new ways and judiciously chosen geometries, which are able to use the benefits of stress-free boundaries.

The existence of instabilities may pave the way for precession becoming a viable way to produce a dynamo effect, but it does not finally answer the question for their effectiveness in generating a magnetic field. Experiments by [Gans, 1971] with a sodium filled cylinder $25 \mathrm{~cm}$ in diameter were able to amplify an applied magnetic field, albeit no self-excitation without it and therefore no dynamo action was noted. Since the mechanical limitations made it difficult to reach more promising parameters, experiments tended to built upon more constrained, basic concepts for reliable dynamos. The Riga experiment [Gailitis et al., 2000] was able to produce a self-exciting dynamo by simulating the theoretically proven Ponomarenko dynamo. Similarly the dynamo by Roberts was used as foundation for the also successful experiment in Karlsruhe [Müller et al., 2006, Müller et al., 2008] with a mesh of cells with guiding tubes. The Von Kármán Sodium (VKS) dynamo [Berhanu et al., 2007, Monchaux et al., 2007] was brought to fruition with more freedom in flow, as its setup was a simple hollow cylinder with two counterrotating impellers at top and bottom. This led to a more turbulent flow with more complex features and of course a more complex effects like field reversals[Pétrélis et al., 2009]. After this, the time was right for a renewed attempt for a purely homogeneous dynamo without any assisting measures to guide the flow except the precessing container similar to the work of Gans. This is currently done at the facility of DRESDyn next to other experiments examining magnetohydrodynamics effects like the magnetorotational instability (MRI) and Taylor instability (TI) [Stefani et al., 2015, Stefani et al., 2017]. In comparison to Gans' experiment at DRESDyn a sodium filled cylinder of $2 \mathrm{~m}$ in height and diameter will rotate around its main axis with a frequency of $10 \mathrm{~Hz}$ and $1 \mathrm{~Hz}$ around its precession axis trying to reach a magnetic Reynolds number of $\mathrm{Rm}=\mu_{0} \sigma \omega_{D} R^{2} \approx 700$ with the magnetic permeability 
$\mu_{0}$, electric conductivity of sodium $\sigma$, radius $R$ and a rotation rate $\omega_{D}$ while achieving an Ekman number of $\mathrm{Ek}=\frac{\nu}{\omega_{D}(2 R)^{2}} \approx 10^{-8}$ with viscosity $\nu$. Even before the finalisation naturally the question occurs: "Does this suffice to reach a dynamo effect?" or respectively "How to find reasonable parameters, where this system will tend to generate fields?" The freedom of the flow reduces the predictability of possible effects and magnetic field generation in contrast to experiments as in Riga and Karlsruhe with their reduced focus. This opens the possibilities for advanced numerical simulations accompanying the experimental setup to examine the complex possible flows and magnetohydrodynamic effects that may be important. There are numerical simulations trying to assess the possibility of dynamo action in precessing spheres [Tilgner, 2005] and cylinders [Nore et al., 2011, Cappanera et al., 2016], suggesting the magnetic Reynolds number of DRESDyn's precessing experiment is sufficient. The Ekman number of these simulations is about four magnitudes above the number of the experiment only offering extrapolations of the actual simulations down to the experimental parameter space. Additionally, for numerical simulations with $\mathrm{Ek} \geq 10^{-4}$ the magnetic Prandtl number $\operatorname{Pm}=\frac{\nu}{\lambda}$ with magnetic diffusivity $\lambda$, where dynamo action starts, is usually above one in simulations and therefore more receptive for viscous dissipation than ohmic. However, the prediction for lower Ekman numbers is a magnetic Prandtl number well below one and new effects are possible. Hence, new ways to push for lower Ekman numbers in numerical simulations are necessary. In this work, the adaption of classic numerical computation techniques for use with General-purpose computing on graphics processing units (GPGPU) is examined to reach for low Ekman numbers while obtaining results in a large parameter space. This is done by using a cuboid container instead of the usually used axisymmetric ones. It guarantees an easy environment for calculations on GPGPUs, while reducing the possibility of viscous boundary effects since this geometry allows for free-slip boundary conditions without loosing the drive for motion inside the fluid. This enables to sweep a broad range of parameters and to obtain insights into precessional instabilities and dynamics inducing magnetic fields into the flow. The main objective is the possible generation of magnetic field, hence the Lorentz force is eliminated. It would feed dynamics of magnetic fields strong enough back into the flow and is important for established magnetic fields. However, the magnetic fields are weak in the initial system and the Lorentz force can be neglected, ending up in what is called a kinematic dynamo.

Chapter 2 displays the methods used to simulate the magnetohydrodynamic system. To this belong the basic equations governing the flow and the magnetic field and the 
computational methods detailing the DNS and the setup of working free-slip boundary conditions. In Chapter 3 the results of purely hydrodynamic simulations in a cubic system ranging from $\mathrm{Ek}=1.0 \cdot 10^{-3}$ to $\mathrm{Ek}=1.0 \cdot 10^{-5}$ are shown and different occurring instabilities (triad resonances, axisymmetric modes and the single vortex state for $\mathrm{Ek} \leq 1.0 \cdot 10^{-4}$ ) are identified and examined. Chapter 4 expands the findings of chapter 3 into magnetohydrodynamic regime by adding a magnetic field influenced by the flow. Since the feedback of the magnetic field due to the Lorentz force is disabled in the simulation, the results here describe only the initial generation of a field but no long-term dynamics. The resulting critical threshold values are defined by different aspects of the physical reality of experiments and theory of precessing fluids and analyzed for their effectiveness as dynamos. The shape of the container is varied slightly in Chapter 5 by using cuboids instead of a cube with edges of equal length as before. The precise ratio of edge lengths is carefully chosen to maximise instabilities. The simulations consider purely hydrodynamic and magnetohydrodynamic cases. The underlying numerical simulations is adapted in Chapter 6 to reproduce the setup of the Herzenberg dynamo[Herzenberg, 1958] within cuboid containers. Chapter 7 discusses the findings of all results gained in previous chapters and analyzes them in the backdrop of precession experiments. 


\section{Methods}

\subsection{Mathematical model}

From the point of view of numerical simulations, the study of precession driven dynamos is not as advanced as other systems with differing driving mechanisms as it is the case with convection based dynamos. The cause of this lies within the special predicaments such a mechanical driven system finds: Since the movement of the walls is fundamental to a preceding system, the container geometry and boundary conditions must be chosen wisely. A sphere seems a logical geometry, but obviously can only be driven by walls satisfying no-slip boundary conditions. These conditions subsequently generate a boundary layer known as Ekman layer [Greenspan, 1969, Ekman, 1905], which thickness of $\mathcal{O}\left(\mathrm{Ek}^{\frac{1}{2}}\right)$ has to be resolved by any numerical method and therefor impedes the examination of low Ekman numbers. Additionally, for an ideal fluid a simple solidbody rotation is the trivial solution for spheres and because of its stability will not produce any fluid motions eligible for dynamo action except for viscous effects from the boundaries [Lorenzani and Tilgner, 2001]. The first obvious deviation from a perfect sphere is an ellipsoid. Here, an ideal fluid will maintain a flow which is known as Poincaré solution [Poincaré, 1910] and introduces a strain into the flow. This causes the flow to become inertially unstable [Kerswell, 1993]. This is of course of interest, since astronomical objects tend to take ellipsoidal shapes and is one possible mechanism in this field. But this means numerically, that the problematic no-slip boundary condition can be exchanged with less demanding conditions like simulated transients [Lorenzani and Tilgner, 2003] or special boundary conditions [Wu and Roberts, 2009]. After all, these studies always relied on using containers symmetric to the axis of rotation, where fluid motion in a state of equilibrium can be viewed as stationary state with a non-trivial motion [Lorenzani and Tilgner, 2003]. Hence, the choice for a nonaxisymmetric container may be a good choice to develop a system, which neglects the viscous coupling with the walls, but can drive a flow by exerting pressure forces via 
the moving walls. Here, a cube or cuboid structure was chosen, since the walls align perfectly with cartesian coordinates, and discretized on a simple grid, appropiate for GPU-computing (see sec. 2.2). The corners of the rectangular base may help stirring the fluid and so support more efficiently the generation of dynamos while the boundaries only satisfy a stress-free flow reducing the effort needed there. The flow on the other hand will show common features of precssing systems, while the system maintains a spherical-like appearance.

\subsubsection{Hydrodynamic Equations of Motion}

Assume a cubic container like in fig. 2.1 with edge length $L$ filled with a fluid of density $\rho$ and viscosity $\mu$. This container rotates with angular frequency $\widetilde{\omega}_{D}$ around an axis normal to one of his sides whereas it precesses with angular frequency $\widetilde{\Omega}_{P}$ with angle $\alpha$ between rotation and precession axis. In this case, $L$ is the obvious choice as specific unit of length for nondimensionalization and

$$
\tau=\frac{1}{\widetilde{\omega}_{D}+\widetilde{\Omega}_{P} \cos \alpha}
$$

as specific unit of time, since it represents the summed rotation around the main rotation axis. Using this for angular velocities, the non-dimensional counterparts are

$$
\begin{aligned}
\omega_{D} & =\tau \widetilde{\omega}_{D}=\frac{\widetilde{\omega}_{D}}{\widetilde{\omega}_{D}+\widetilde{\Omega}_{P} \cos \alpha}, \\
\Omega_{P} & =\tau \widetilde{\Omega}_{P}=\frac{\widetilde{\Omega}_{P}}{\widetilde{\omega}_{D}+\widetilde{\Omega}_{P} \cos \alpha} .
\end{aligned}
$$

The Poincaré number is now

$$
\Omega=\frac{\widetilde{\Omega}_{P}}{\widetilde{\omega}_{D}}=\frac{\Omega_{P}}{\omega_{D}}
$$

and therefore the non-dimensional angular velocities can also be written as

$$
\begin{aligned}
& \omega_{D}=\frac{1}{1+\Omega \cos \alpha}, \\
& \Omega_{P}=\frac{\Omega}{1+\Omega \cos \alpha} .
\end{aligned}
$$

At this point, incompressibility is assumed in this system, since it is a good choice for most precessing systems like the DRESDYN experiment, but later we will adapt this to a more fitting approximation. The equation of motion for the (non-dimensional) velocity $\boldsymbol{u}(\boldsymbol{r}, t)$ is the well-known Navier-Stokes-equation

$$
\partial_{t} \boldsymbol{u}=\operatorname{Ek}^{2} \boldsymbol{u}-(\boldsymbol{u} \cdot \nabla) \boldsymbol{u}-\nabla p
$$


with pressure $p$ and Ekman number

$$
\mathrm{Ek}=\frac{\nu}{\left(\widetilde{\omega}_{D}+\widetilde{\Omega}_{P} \cos \alpha\right) L^{2}}
$$

with kinematic viscosity $\nu$. Also, because of the incompressibility of the fluid,

$$
\nabla \boldsymbol{u}=0
$$

But this applies to the system viewed from the inertial space, where the boundaries rotate, for calculations it is much more comfortable, to switch to a system, where the boundaries remain fixed. The best form for the coordinate system is a cartesian grid, whose axes are parallel to the different sides of the cube like in figure 2.1. In this system, the rotation vector can be defined as

$$
\omega_{D}=\omega_{D} \cdot \hat{z}
$$

and the precession vector as

$$
\boldsymbol{\Omega}_{P}=\Omega_{P} \cos \alpha \cdot \hat{\boldsymbol{z}}+\Omega_{P} \cdot \boldsymbol{p}(t)
$$

with

$$
\boldsymbol{p}(t)=\sin \alpha\left(\cos \left(\omega_{D} t\right) \hat{\boldsymbol{x}}+\sin \left(\omega_{D} t\right) \hat{\boldsymbol{y}}\right) .
$$

Also, the Navier-Stokes equation sees additional forces, the Coriolis force

$$
\boldsymbol{F}_{C}=-2\left(\boldsymbol{\omega}_{D}+\boldsymbol{\Omega}_{P}\right) \times \boldsymbol{u}=-2\left(\hat{\boldsymbol{z}}+\Omega_{P} \boldsymbol{p}(t)\right) \times \boldsymbol{u}
$$

originating from the rotation and the Poincaré force

$$
\boldsymbol{F}_{P}=\left(\boldsymbol{\omega}_{D} \times \boldsymbol{\Omega}_{P}\right) \times \boldsymbol{r}=\omega_{D} \Omega_{P}(\hat{\boldsymbol{z}} \times \boldsymbol{p}(t)) \times \boldsymbol{r},
$$

which comes into play from the motions of precession. Now, the equation is

$$
\partial_{t} \boldsymbol{u}=\operatorname{Ek} \nabla^{2} \boldsymbol{u}-(\boldsymbol{u} \cdot \nabla) \boldsymbol{u}-\nabla p-2\left(\boldsymbol{\omega}_{D}+\boldsymbol{\Omega}_{P}\right) \times \boldsymbol{u}+\left(\boldsymbol{\omega}_{D} \times \boldsymbol{\Omega}_{P}\right) \times \boldsymbol{r}
$$

Additionally, the Poisson equation needed to calculate pressure $p$ proves difficult to solve for computational solutions. Especially, the strong parallelization GPU-computing introduces to numerical simulations conflicts with the often global approach to solving Poisson equations. Therefore, the method of artificial compressibility [Chorin, 1967] is used and the fluid behaves approximately like a weak compressible one, for whose the maximal velocity $u_{\max }$ fulfills

$$
u_{\max }<<c
$$




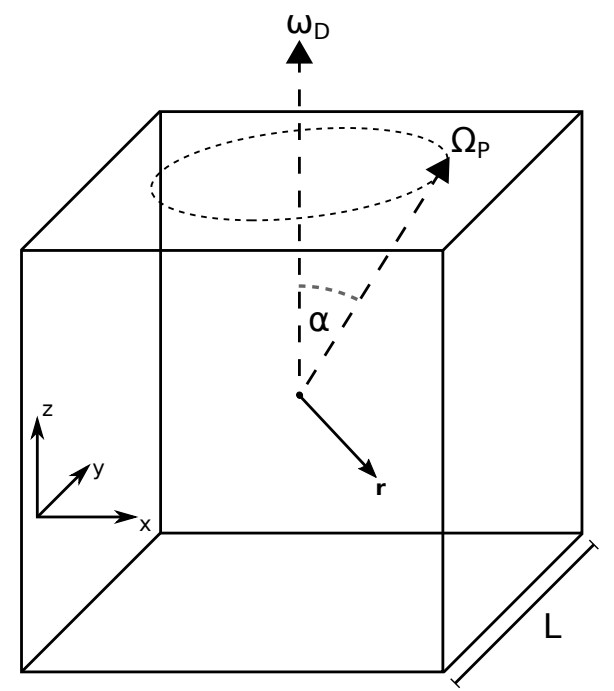

Figure 2.1: Basic geometry used in the simulation: A cube with side length $L$ and a coordinate system with axes parallel to the sides of the cube. The rotation axis $\boldsymbol{\omega}_{D}$ is aligned to the z-axis, while the precession axis $\boldsymbol{\Omega}_{P}$ rotates around it with a fixed angle $\alpha$ between them. The origin lies in the center of the cube, around which the rotation takes place.

with the speed of sound $c$. In the consequence, the equation of state

$$
\Delta p \approx c^{2} \Delta \rho
$$

is valid and the Navier-Stokes equation changes to

$$
\partial_{t} \boldsymbol{u}=\operatorname{Ek} \nabla^{2} \boldsymbol{u}-(\boldsymbol{u} \cdot \nabla) \boldsymbol{u}-c^{2} \nabla \rho-2\left(\hat{\boldsymbol{z}}+\Omega_{P} \boldsymbol{p}(t)\right) \times \boldsymbol{u}+\omega_{D} \Omega_{P}(\hat{\boldsymbol{z}} \times \boldsymbol{p}(t)) \times \boldsymbol{r}
$$

with a modified mass conservation

$$
\nabla \boldsymbol{u}+\partial_{t} \rho=0
$$

The equations in this form are used in the numeric simulation and were already proven useful in applications for rotating convection driven dynamos [Tilgner, 2012, Tilgner, 2014].

\subsubsection{Induction equation}

If magnetic fields are included into simulations, the magnetic field $\boldsymbol{B}$ will be governed by the induction equation

$$
\partial_{t} \boldsymbol{B}+\nabla \times(\boldsymbol{B} \times \boldsymbol{u})=\frac{\mathrm{Ek}}{\mathrm{Pm}} \nabla^{2} \boldsymbol{B} .
$$


Ek is defined as in equation 2.8, while the definition of the magnetic Prandtl number is $\mathrm{Pm}=\frac{\nu}{\lambda}$ with $\lambda$ as the magnetic diffusivity. Of course, $\boldsymbol{B}$ will be solenoidal, so

$$
\nabla \cdot \boldsymbol{B}=0
$$

Hypothetically, the Lorentz force-term as defined as

$$
\boldsymbol{F}_{B}=(\nabla \times \boldsymbol{B}) \times \boldsymbol{B},
$$

would be added to the Navier Stokes equation (see eq. 2.18) to assess the full magnetohydrodynamic problem. Instead this work investigates it as kinematic dynamo problem, which foremost evaluates whether a flow can support a magnetic field at all. This will be further explained in section 4 .

\subsection{Computational methods}

Since the used computation hardware is a GPU-computing system, the software code has to emphasize the special advantages of such a system. Hence, the code has to be highly parallelized, with a minimum on communication between the threads and usage of memory. The ideal choice for this is a finite differences method on a cartesian grid. The regularity of the grid implies a location-independent calculation, whereas an explicit third order Runge-Kutta time step needs minimal data for every single data point. For diffusion and induction terms of the magnetic, density and velocity field a fourth order central differences scheme was applied, and upwind biased third order finite differences for advection terms.

\subsubsection{Boundary conditions}

The boundaries correlate to the mantle and therefore the fluid is contained to a volume with $-\frac{L_{x}}{2}<\widetilde{x}<\frac{L_{x}}{2},-\frac{L_{y}}{2}<\widetilde{y}<\frac{L_{y}}{2},-\frac{L_{z}}{2}<\widetilde{x}<\frac{L_{z}}{2}$ with, in the case of a cube, $L=L_{x}=L_{y}=L_{y}$ and nondimensionalized $-\frac{1}{2}<x<\frac{1}{2},-\frac{1}{2}<y<\frac{1}{2},-\frac{1}{2}<x<\frac{1}{2}$. For the velocity field, this means naturally the existence of a no-flux condition at the boundaries, i.e. the normal component vanishes. Additionally, the velocity is subject to free-slip boundary condition, the normal derivatives of parallel components vanish at the boundaries. So, summarized

$$
\begin{aligned}
v_{\text {normal }}(\text { boundary }) & =0, \\
\partial_{\text {normal }} v_{\text {parallel }}(\text { boundary }) & =0 .
\end{aligned}
$$




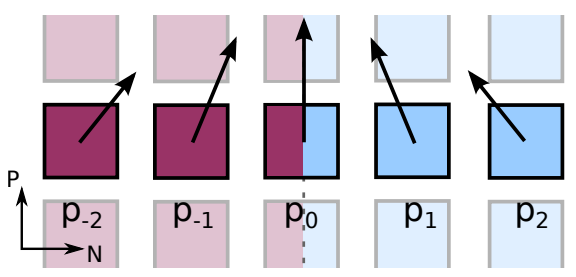

Figure 2.2: The graphical representation of free-slip boundary condition at $p_{0}$ with points in bright blue within the fluid volume and points in dark magenta outside of it. $p_{0}$ itself play a special role as the point on the border and $p_{-1}$ and $p_{-2}$ are symmetric halo points which imitate a stress-free flow parallel $(\mathrm{P})$ to the boundary and no flux normal $(\mathrm{N})$ to it.

The use of free-slip boundary conditions reduces the need of higher resolution at the borders to compute boundary layers, as it would be necessary with no-slip conditions. The magnetic field is also contained inside the volume and no field outside the boundaries will be calculated to save calculation time. Instead, a boundary condition is imposed, that approximates a vacuum outside the boundaries and is better known as pseudovacuum boundary condition. Here, the tangential component vanishes while the normal derivative of the normal component equals zero, so that the magnetic field is divergence free. To create this conditions, additional 'halo' points were added outside the grid calculating the fluid, so that two additional layers on every side exist. Grid points alongside a specific axis could be counted as $p_{0}, p_{1}, p_{2}, \ldots p_{N-3}, p_{N-2}, p_{N-1}$, where $N$ is the specific number of points in this direction and $p_{0}$ and $p_{N-1}$ lie on the boundary. Now, new points $p_{-2}, p_{-1}, p_{N}, p_{N+1}$ are added, whose field values will be defined specific to the type of boundary condition. To imitate a boundary condition where the value at point $p_{0} v\left(p_{0}\right)$ has to be zero, the values of the halo points have to be set to the negative value of their mirroring points on the other side of the boundary, $v\left(p_{-1}\right)=-v\left(p_{1}\right)$ and $v\left(p_{-2}\right)=-v\left(p_{2}\right)$ or in the same manner $v\left(p_{N+2}\right)=-v\left(p_{N-2}\right)$ and $v\left(p_{N+1}\right)=$ $-v\left(p_{N-1}\right)$ at $p_{N-1}$. A vanishing normal derative is easily introduced by setting the corresponding values on the halo points $v\left(p_{-1}\right)=v\left(p_{1}\right)$ and $v\left(p_{-2}\right)=v\left(p_{2}\right)$ (see fig. $2.2)$ or $v\left(p_{N+2}\right)=v\left(p_{N-2}\right)$ and $v\left(p_{N+1}\right)=v\left(p_{N-1}\right)$, respectively. For example, a freeslip condition for the velocity field on a plane normal to the $x$-axis sets halo points with velocities $\boldsymbol{v}\left(p_{-1}\right)=\left(-v_{x}\left(p_{1}\right), v_{y}\left(p_{1}\right), v_{z}\left(p_{1}\right)\right)$ and $\boldsymbol{v}\left(p_{-2}\right)=\left(-v_{x}\left(p_{2}\right), v_{y}\left(p_{2}\right), v_{z}\left(p_{2}\right)\right)$. This allows for a completely nonlocal computation of every grid point, greatly enhancing the GPU processing. 


\section{Hydrodynamics}

For a purely hydrodynamic flow (where no magnetic field or Lorentz force were calculated) the numeric methods allow for a huge range of values to be processed: At an angle $\alpha=60^{\circ}$, for Ekman numbers from $10^{-3}$ to $10^{-5}$ simulations were done with varying precession rates $\Omega_{P}$ from -0.02 to -0.3 . The simulation focused on purely retrograde precession since former studies showed, that they were more prone to instabilities [Lorenzani and Tilgner, 2001, Tilgner and Busse, 2001]. The starting velocity field consists of random vectors with components varying between $\left[-10^{-4}: 10^{-4}\right]$, while the density $\rho=1$ everywhere. The speed of sound $c$ for eq. 2.18 was chosen such that $c^{2}=500$ and $u<0.04 c$ is satisfied at every grid point inside the container for any run in the following chapter. Exemplary simulation runs with $c^{2}=1000$ and $c^{2}=5000$ did not achieve any notable change in significant statistic variables like $E_{k i n}$

or $E_{a}$ compared to runs with $c^{2}=500$, so the latter is the reasonable choice for further simulations. Some results discussed here and in chapter 4 are already referenced in [Goepfert and Tilgner, 2016] and [Goepfert and Tilgner, ewed].

\subsection{Structure}

The flow structure in axisymmetric containers like cylinders and spheroids are well documented and analytical solutions exist to describe the eigenvalues and -modes - known as inertial modes or Kelvin modes[Greenspan, 1969]. Unfortunately, no analytical solution is known for non-axisymmetric cuboid boundaries. But one can naively expect that the main flow will behave in a similar manner as it does in spheroids and cylindrical containers. Independently from the boundaries, seen from an inertial reference frame, the main response of ideal fluids to weak precession of the container, as it is to any other small disturbance of rotation, is to keep rotating around the former rotation axis. In the mantle reference system, which is used in the calculation of the equation of motion in chapter 2.1.1, this occurs as a fluid motion around an axis other than the rotation 


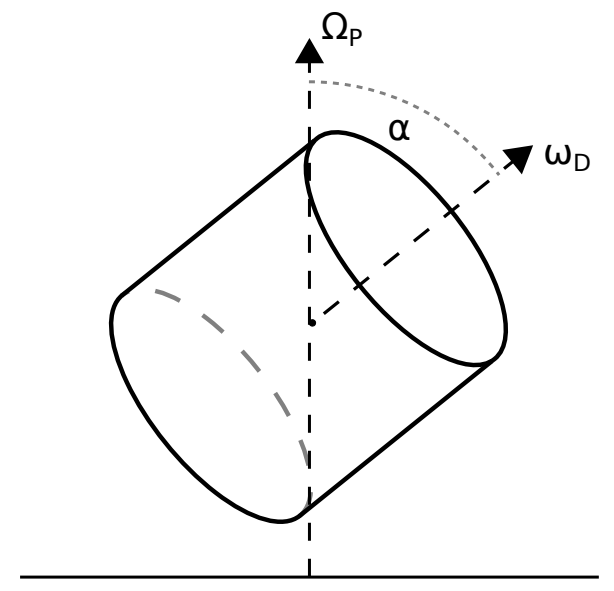

Figure 3.1: Sketch of a hypothetical experimental setup: The container is mounted on a table, so that it is tilted to $\alpha$. The table rotates with $\boldsymbol{\Omega}_{P}$, while the container itself rotates with $\omega_{D}$.

axis and is obviously proportional to $\left(\boldsymbol{\omega}_{D}+\boldsymbol{\Omega}_{P}\right) \times \boldsymbol{r}$ as defined in eq. 2.10 and 2.11. In the equation of motion (eq. 2.18) this contributes as the Poincare force $\boldsymbol{F}_{P}$. The time dependent part in $\boldsymbol{\Omega}_{P}, \boldsymbol{p}(t)$ (see eq. 2.12), agitates an inertial mode usually called the spin-over or tilt-over mode. Moving inside the boundaries with the same rate as $\boldsymbol{p}(t)$, $\omega_{D}$, would be time-independent for axisymmetric container in the precession reference frame. In this frame, the boundaries rotate with their fixed rotation, while the reference frame rotates with the motion of the precession. More illustrative in this context is how it is represented in experiments: The container rotates around its axis tilted at angle $\alpha$, while sitting on a table itself rotating with $\Omega_{P}$ to induce the precession as pictured in fig. 3.1. The precession reference system is fixed on the table, only experiencing the standard rotation of the container. The precession axis is fixed in this system and the spin-over mode is therefore time-independent. The latter is obviously only true for axisymmetric containers, since the boundaries do not change the position there. But while the moving boundaries in cuboids interfere with it, the spin-over mode does exist and behaves like described before. This can be seen in fig. 3.2. The velocity $u$ in the precession frame forms a distinct s-curve, which is known from experiments inside precessing water-filled cylinders [Mouhali et al., 2012, Herault et al., 2015]. In these cases, with cylindrical container, the inertial modes take the form $u_{m n k}(\boldsymbol{r}, t) \propto \mathrm{e}^{i\left(\omega_{m n k} t\right)+m \phi+k z}$ with $\omega_{m n k}$ describing the rotation rate of it, and $m, n, k$ as the azimuthal, radial and axial wave numbers. The spin-over mode in this system is identified as a mode with $m=1$ and $k=1$ and of course $\omega_{m n k}=\omega_{D}$. With no analytical solution, no such exact 

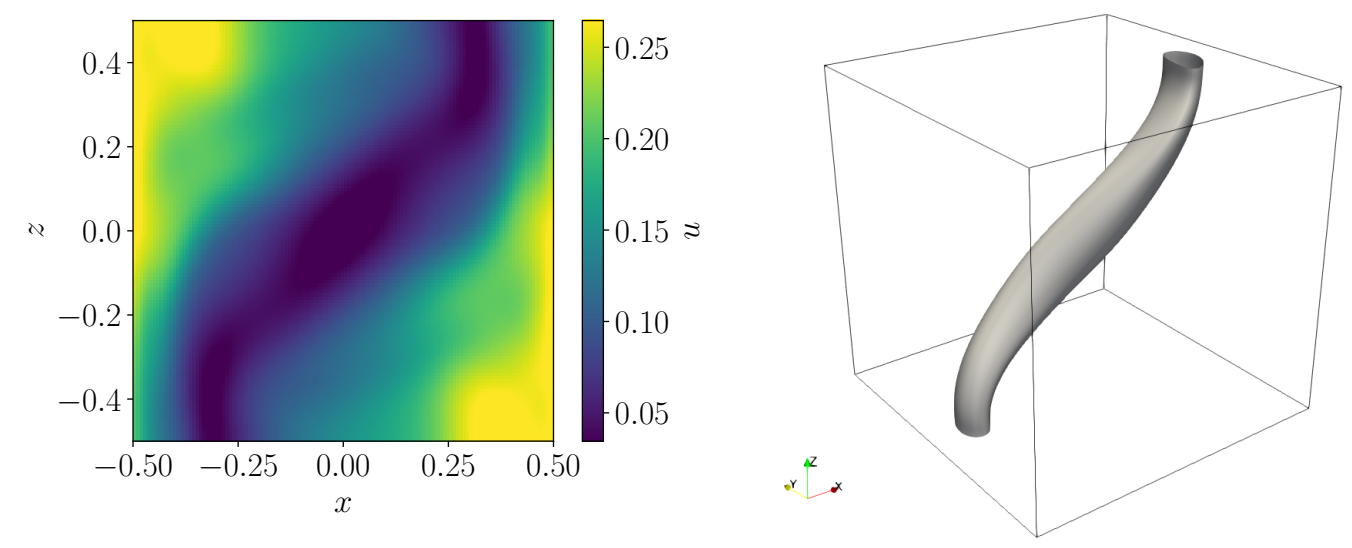

Figure 3.2: The velocity $u$ in the precession reference frame at $\mathrm{Ek}=2.5 \cdot 10^{-4}, \Omega_{P}=$ -0.04 in a plane spanned by $\boldsymbol{\omega}_{D}$ and $\boldsymbol{\Omega}_{P}$ (left) and as an isosurface at $u=0.05$ (right): In both figures the s-curve caused by the spin-over-mode is clearly visible.

separation into modal structures is possible. But the emerging modes can be intuitively guessed by visual cues and categorized by analogous wave numbers.

\subsubsection{Kinetic Energy}

The kinetic energy in the system can be calculated as $E_{k i n}=\frac{1}{V} \sum_{i} V_{i, \text { cell }} \tilde{u}_{i}^{2}$ with the volume $V_{i, c e l l}$ of grid point $i$ and $\tilde{u}_{i}$ as the respective velocity in the mantle reference frame. It will grow fast from the starting conditions by the means of the energy introduced by the Poincare term, but saturates roughly after a starting phase (see fig. 3.3). Since the energy introduced into the system is dependent on the Poincare term in eq. 2.14 , a higher precession rate $\left|\Omega_{P}\right|$ should generate a higher saturation energy in the system. In figure 3.4 the kinetic energy of fully developed flows for four different Ekman numbers and precession rates from -0.3 to 0.0 is shown, averaged over at least a time span of $\Delta t=2000$. For all values of Ek the kinetic energy grows with increasing $\left|\Omega_{P}\right|$ like expected, however the growth is not continuous: At Ek $=2.5 \cdot 10^{-4}$ and $1.0 \cdot 10^{-4}$ the energy slowly grows at low precession rates but at a critical precession rate a jump in $E_{k i n}$ occurs. This behavior is noted at different Ek and the curve of the associated critical precession rate is visualized in fig 3.5 Here we see, that this seems not to be the case for $\mathrm{Ek}=1.0 \cdot 10^{-5}$ anymore, where the energy grows smoothly. 


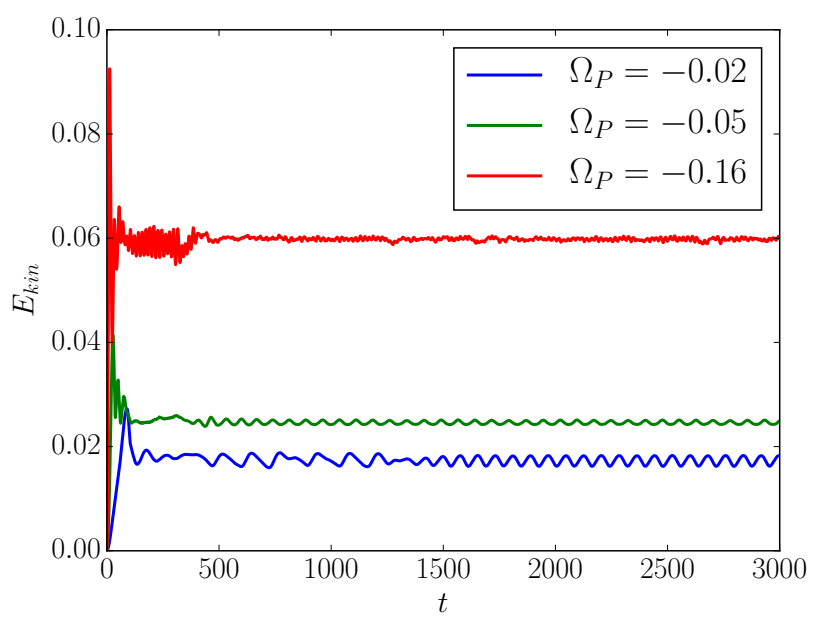

Figure 3.3: kinetic energy $E_{k i n}$ over time $t$ for exemplary precession rates $\Omega_{P}=$ $-0.02 ;-0.05 ;-0.16$ at $E k=2.5 \cdot 10^{-4}$ and $\alpha=60^{\circ}$ : The energy grows fast at the beginning, but saturates around a energy value specific for the precession rate.

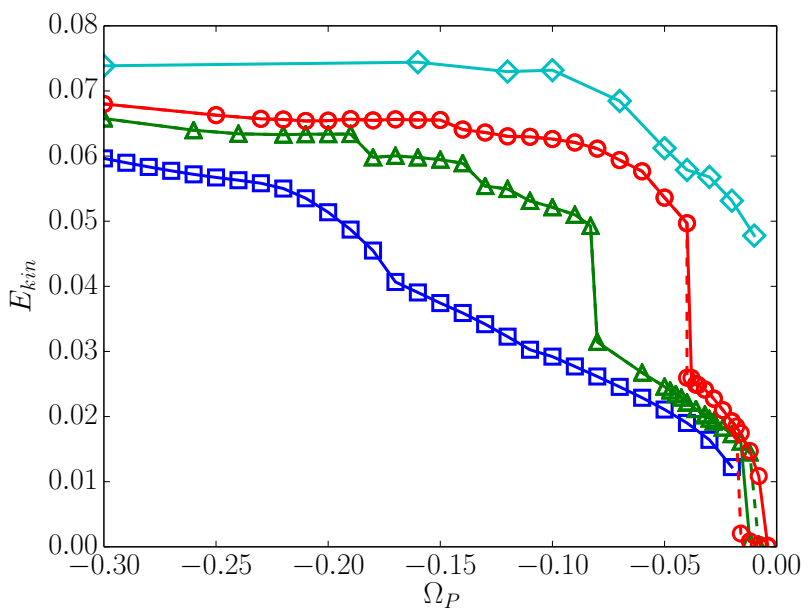

Figure 3.4: Kinetic energy $E_{k i n}$ for different precession rates $\Omega_{P}$ at Ekman numbers $\mathrm{Ek}=1.0 \cdot 10^{-3}$ (squares); $2.5 \cdot 10^{-4}$ (triangles); $1.0 \cdot 10^{-4}$ (circles); $1.0 \cdot 10^{-5}$ (diamonds): The energy increases with increasing absolute precessing rate smoothly. At Ek $=2.5 \cdot 10^{-4}$ and $1.0 \cdot 10^{-4}$ a strong jump in the energy is seen. A dashed line indicates hysteresis effects: It is obtained by using a starting field from a preceding simulation with a lower absolute value, while the continuous line marks the reverse direction. 


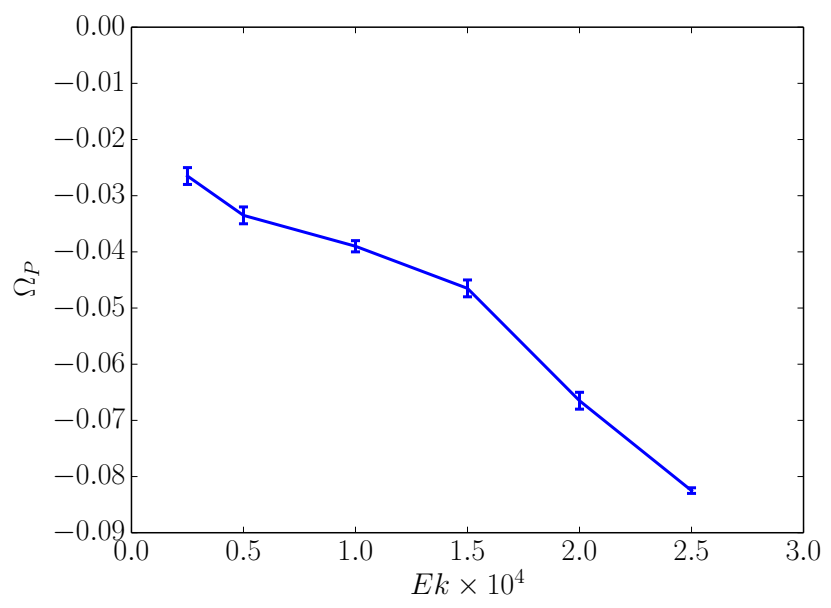

Figure 3.5: Jump in $E_{k i n}$ for a critical precession rate $\Omega_{P}$ : The transition occurs at the marked line

\subsection{Radial vorticity distribution}

To further understand the structure of the flow, a vorticity dependent on the radius $r$ is defined as

$$
\boldsymbol{\omega}(r)=\frac{1}{V_{r}}\left\langle\int_{V_{r}} \frac{1}{2} \nabla \times \boldsymbol{u} \mathrm{d} V\right\rangle+\omega_{D} \cdot \hat{\boldsymbol{z}}
$$

with $V_{r}$ as the intersection of the cube and a shell of radius $r$ and thickness $\Delta r=$ $\min \left(N_{x}, N_{y}, N_{z}\right)^{-1}$ as shown in fig. 3.6. The definition of $\Delta r$ guarantees the maximal radial resolution and enough independent data points in every set $V_{r}$. The additional $\omega_{D} \cdot \hat{z}$ transforms the rotation from the mantle system to the precession reference system. Here the container rotates with $\omega_{D} \hat{\boldsymbol{z}}$, but the fluid rotation deviates more with increasing precession $\Omega_{D}$, producing a more distinguishable picture than it would do in the mantle frame with the additional rotational component introduced by the Coriolis force. Because of the moving boundaries in this system or the time dependent precession axis in the mantle reference system, the time average $\langle\ldots\rangle$ is necessary for every case, even the ones with a laminar flow, since the rotation axis of the fluid will be dependent on the position of the boundaries and the precession axis and will never be truly time independent. In figures $3.7 \mathrm{a}, 3.7 \mathrm{~b}$ and $3.7 \mathrm{c} \boldsymbol{\omega}(r)$ is split into the magnitude $\omega(r)$ and the angle $\theta(r)$ between it and the rotation axis $\omega_{D}$ using contour plots illustrating a phase space with parameters $r$ and $\Omega_{P}$ for $\mathrm{Ek}=1.0 \cdot 10^{-3}, \mathrm{Ek}=2.5 \cdot 10^{-4}$ and $\mathrm{Ek}=1.0 \cdot 10^{-4}$. Figs. $3.8 \mathrm{a}, 3.8 \mathrm{~b}$ and $3.8 \mathrm{c}$ represent the same but for selected values of $\Omega_{P}$ at $\mathrm{Ek}=2.5 \cdot 10^{-4}$, 

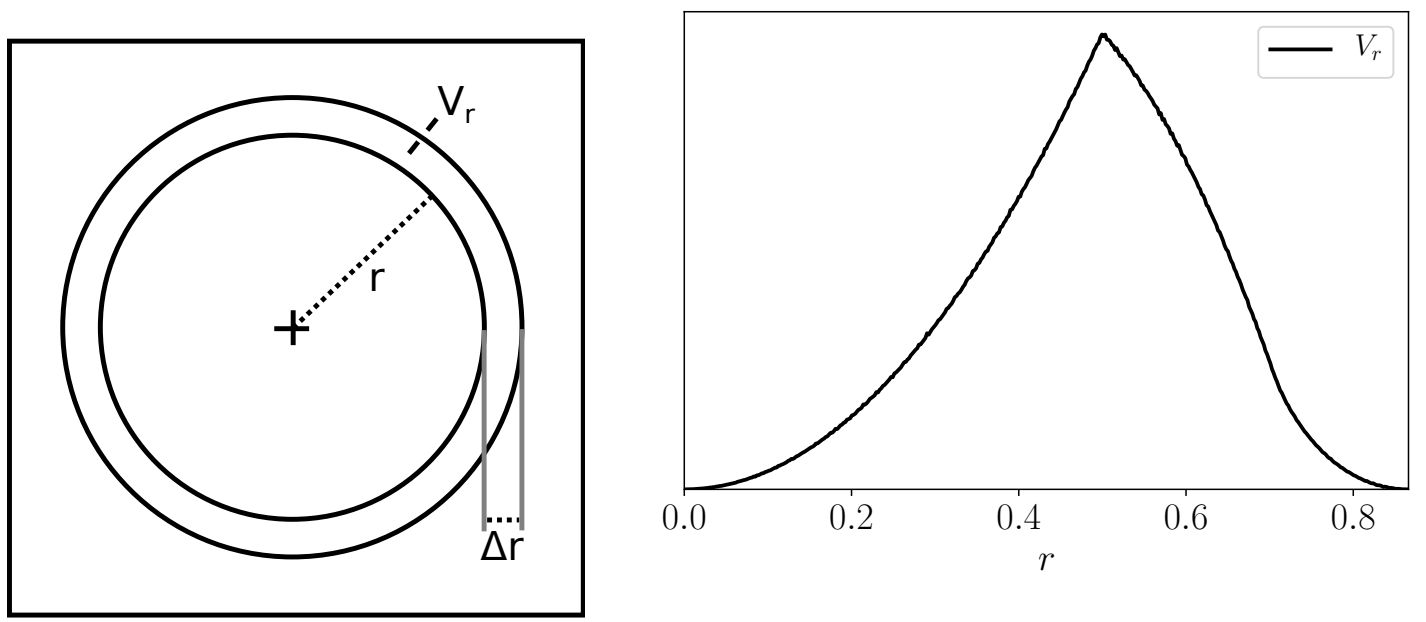

Figure 3.6: Definition of the radial distribution: On the left side, the composition of $V_{r}$ is pictured: a spherical shell of radius $r$ and thickness $\Delta r$ wrap around the specific volume for $\boldsymbol{\omega}(r)$. In numeric context all grid points within the shell will be summed up, weighted with the volume they associated with (see sec. 2.2). Of course, with growing $r, V_{r}$ will grow too, but when it starts to intersect with sides of the cube at $r \approx 0.5$, the volume will decrease for bigger $r$, until it does not intersect with the cube anymore at $r \approx 0.5 \sqrt{3} \approx 0.87$ as seen on the right side. This implies that in radial distributions values at $r \approx 0$ and $r>0.6$ will not be as important in mean values over the whole cube volume as they appear in the distribution.

$\mathrm{Ek}=1.0 \cdot 10^{-4}$ and additionally $\mathrm{Ek}=1.0 \cdot 10^{-5}$. For the last Ek the range of simulated $\Omega_{P}$ was not broad enough to illustrate it in a contour plot. At this point one has to keep in mind that at $r=0.5$ the spherical nature of the radial distribution intersects with the sides of the cube and any analogy with a sphere can only go so far since interaction with the boundaries will dominate beyond this point. Values at $r>0.5$ only describe the behavior in the corners. Now looking at the relatively high $\mathrm{Ek}=10^{-3}$ in figure $3.7 \mathrm{a}$, it is apparent that the area with homogeneous $\omega(r)$ and $\theta(r)$ is confined to $r \approx 0.3-0.4$. $\theta(r)$ starts to decline until its zero at the outer boundary, due to the boundary condition of the sides which prohibits any non-parallel flow. Overall, with increasing $\left|\Omega_{P}\right|$, the angle increases in the bulk of the fluid and the rotation axis deviates as explained earlier on. The magnitude $\omega(r)$ on the other hand reaches at the outer point the value of $\omega_{D}$, rotating with the boundary caused by the additional $\omega_{D} \hat{z}$-term. Around $r \approx 0.8$ it experiences a maximum, indicating additional shearing in the flow at the farthest 
(a) $\mathrm{Ek}=1.0 \cdot 10^{-3}$
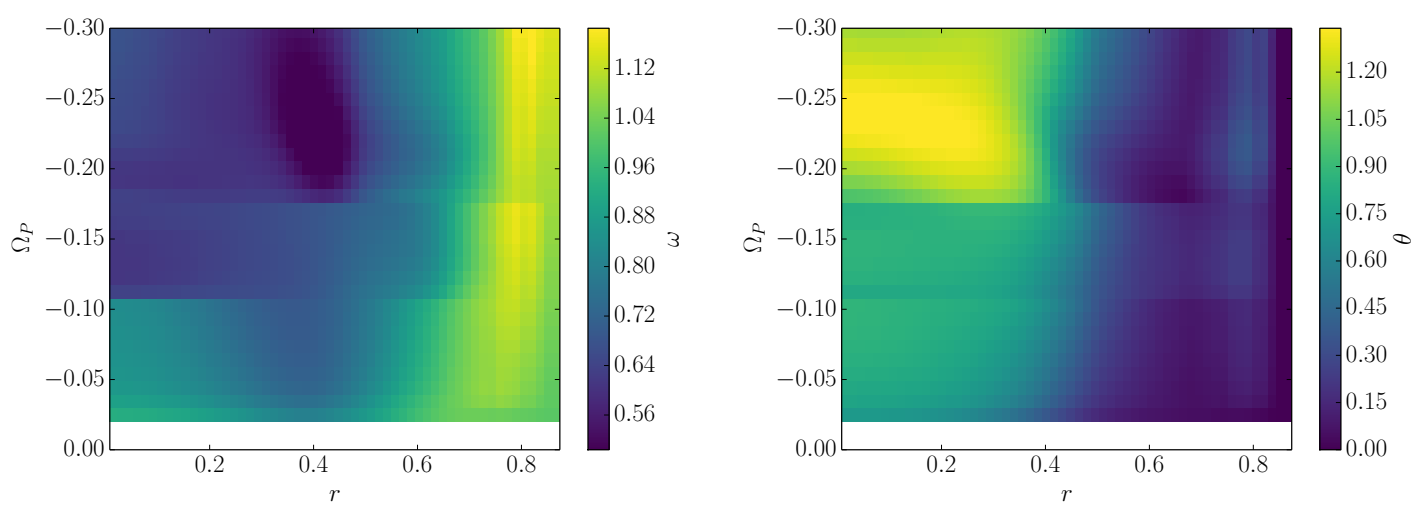

(b) $\mathrm{Ek}=2.5 \cdot 10^{-4}$
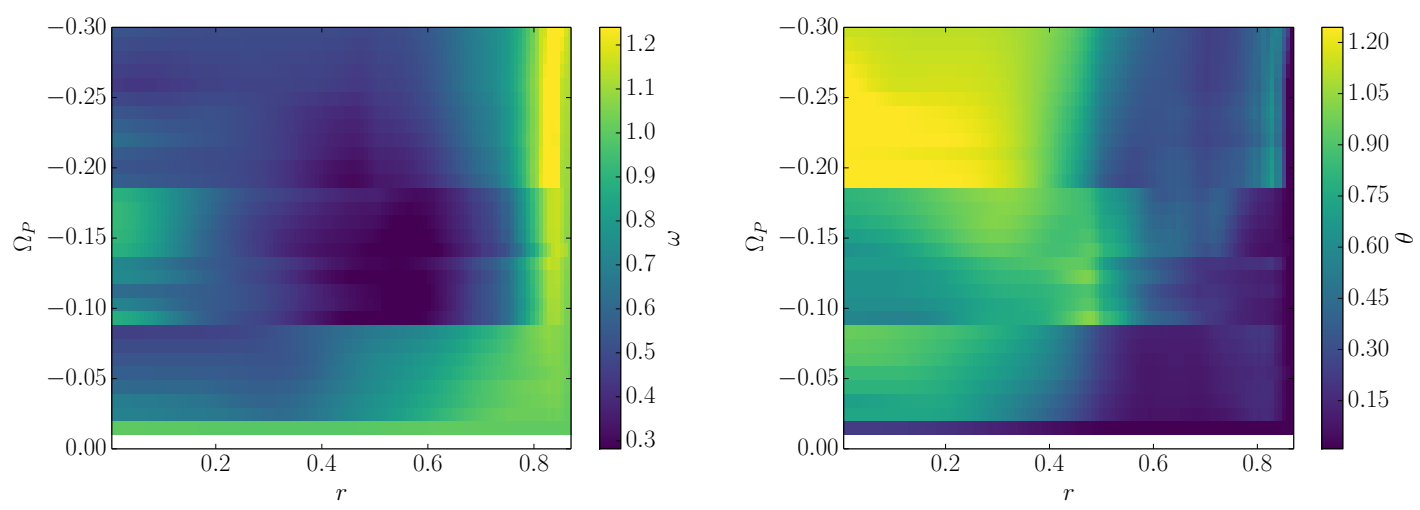

(c) $\mathrm{Ek}=1.0 \cdot 10^{-4}$
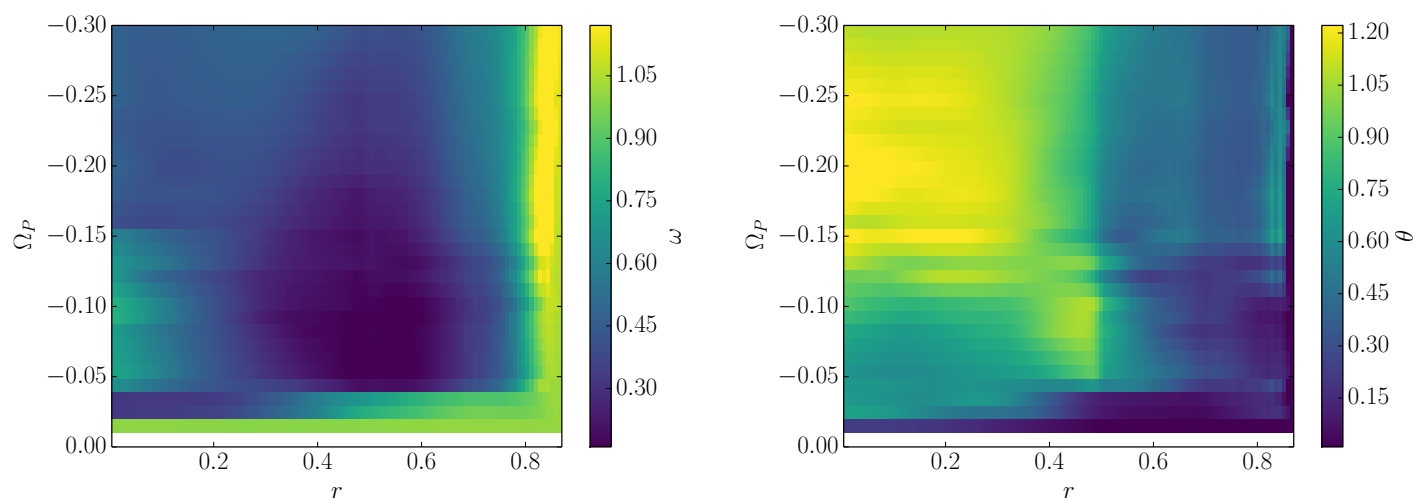

Figure 3.7: $\omega(r)$ (left side) and $\theta(r)$ (right side) for (a) $\mathrm{Ek}=1.0 \cdot 10^{-3}$, (b) $\mathrm{Ek}=2.5 \cdot 10^{-4}$ and (c) Ek $=1.0 \cdot 10^{-4}$ in the $\left(\Omega_{P}, r\right)$-plane. For points in the white area no data were collected. 
(a) $\mathrm{Ek}=2.5 \cdot 10^{-4}$
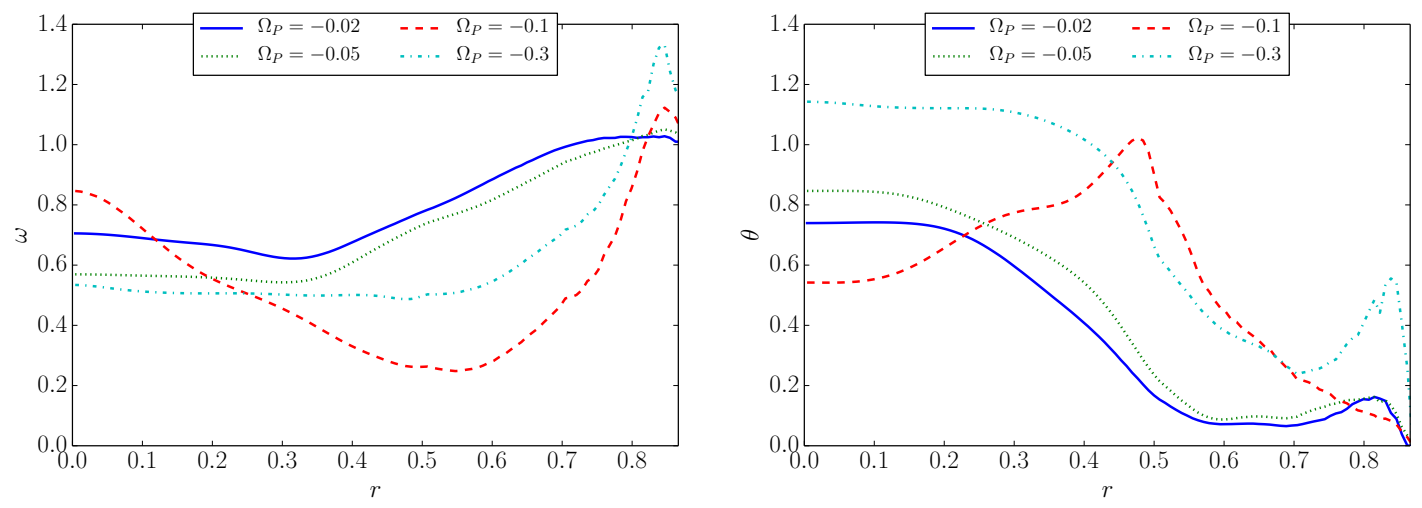

(b) $\mathrm{Ek}=1.0 \cdot 10^{-4}$
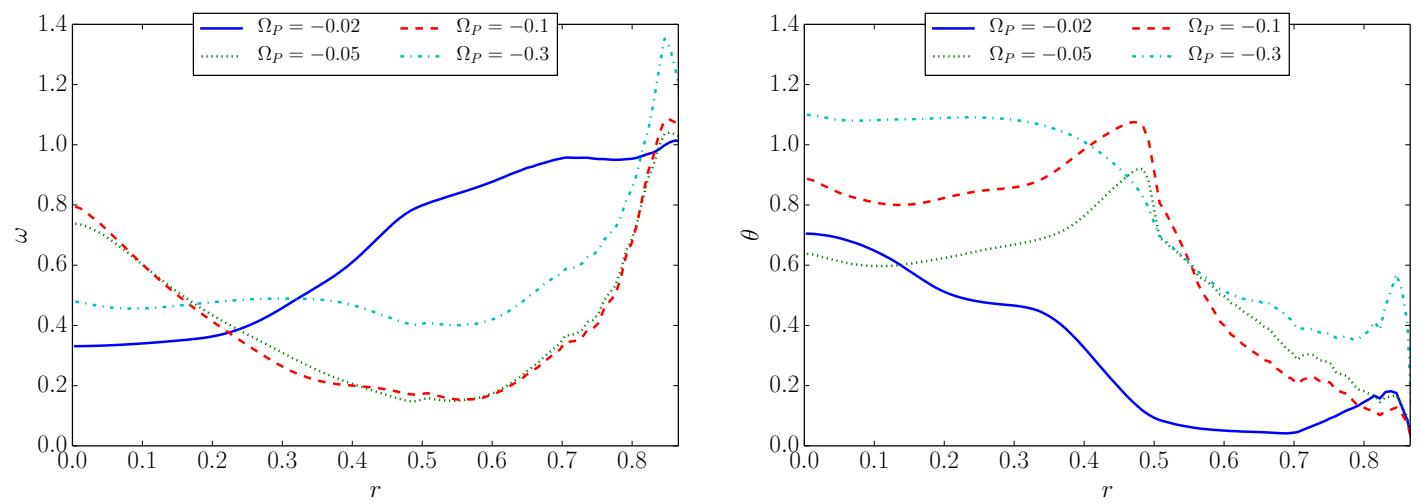

(c) $\mathrm{Ek}=1.0 \cdot 10^{-5}$
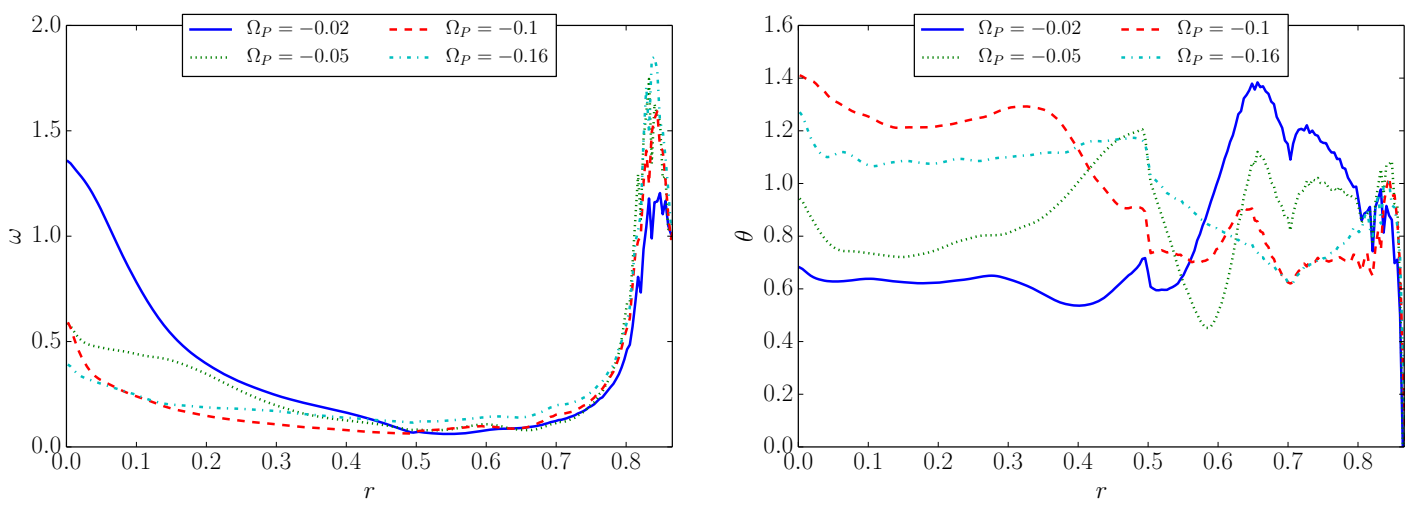

Figure 3.8: $\omega(r)$ (left side) and $\theta(r)$ (right side) for (a) $\mathrm{Ek}=2.5 \cdot 10^{-4}$, (b) $\mathrm{Ek}=1.0 \cdot 10^{-4}$ and (c) Ek $=1.0 \cdot 10^{-5}$ for chosen precession rates $\Omega_{P}$. 
points of the cube. Inwards, the magnitude has a minimum around $r \approx 0.5$, so that the homogeneous part is confined to $r<0.3$ and the effects of the boundaries on the flow are significant outwards. But the most distinctive feature seen here is the distinctive discontinuity separating the contour plot at $\Omega_{P}=-0.1$ and -0.18 into three regions. This indicates a transition in flow structure, which coincide with variation of precession. Further simulations with increasing and decreasing precession rates crossing the specific values of $\Omega_{P}$ show no adherence to any kind of hysteresis, binding the change in structure strictly to a change in geometrical parameters. Also, this behavior occurs at the much more interesting lower Ekman numbers in figures $3.7 \mathrm{~b}$ and $3.7 \mathrm{c}$, at a decreasing $\left|\Omega_{P}\right|$ for the first transition, while the second transition appears at higher $\left|\Omega_{P}\right|$, but vanishes at $\mathrm{Ek}=1.0 \cdot 10^{-4}$. Going further to even lower Ekman numbers, the higher necessary resolution of $N_{X}=N_{Y}=N_{Z}=256$ made it not feasible to simulate as many different precession rates as needed for a continuous $\boldsymbol{\omega}(r)$ contour plot like before, but fig. 3.8c shows a chosen set of parameters. Here we see a new behavior in $\omega(r)$ at $\Omega_{P}=-0.02$ as it starts to get a maximum in the center higher than the actual rotation. This kind of superrotation in the bulk has not been observed at higher Ekman numbers and deserves special recognition later on in section 3.3. $\theta(r)$ on the other hand has only few specific characteristics, it is mainly homogeneous up to $r \approx 0.4$ but strongly fluctuates beyond it. This may originate in chaotic behavior in the corners at this Ekman number. To simplify the overall picture, one can try to take an average of $\boldsymbol{\omega}(r)$ up to a specific $r$ since it may not be constant, this new measurement will show discontinuous transitions more clearly. For this,

$$
\boldsymbol{\omega}_{F}=\frac{3}{4 \pi r_{\text {end }}^{3}} \int_{0}^{r_{e n d}} \boldsymbol{\omega}(r) 4 \pi r^{2} \mathrm{~d} r
$$

is defined with $r_{\text {end }}$ set to 0.1 . The resulting plots for the analogous to $\omega(r)$ and $\theta(r)$ calculated $\omega_{F}$ and $\theta_{F}$ can be seen in figure 3.9. In $\omega_{F}$ the transitions for $\mathrm{Ek}=1.0 \cdot 10^{-4}$ and $\mathrm{Ek}=2.5 \cdot 10^{-5}$ are clearly visible, since the small chosen $r_{\text {end }}$ highlights the high values in $\omega(r)$ inside the middle region. Choosing a larger $r_{\text {end }}$ subdues this difference a little as seen in fig. 3.10 with $r_{\text {end }}=0.3$. A closer look at the mean flow like in fig. 3.11 helps to explain the transitions: Since the flow is never time independent, the mean flow is calculated by averaging over points in time with the same specific geometrical position of the rotation axes inside the mantle frame, respectively when the precession axis $\Omega_{P}$ finishes a full rotation in the mantle reference system. Therefor the average was about states at $t=\frac{2 \pi}{\omega_{D}} n$ with $n \in \mathbb{N}$. Now, in this figure the velocity is projected on the shown plane, displaying a simple rotation inside the Cartesian system around 

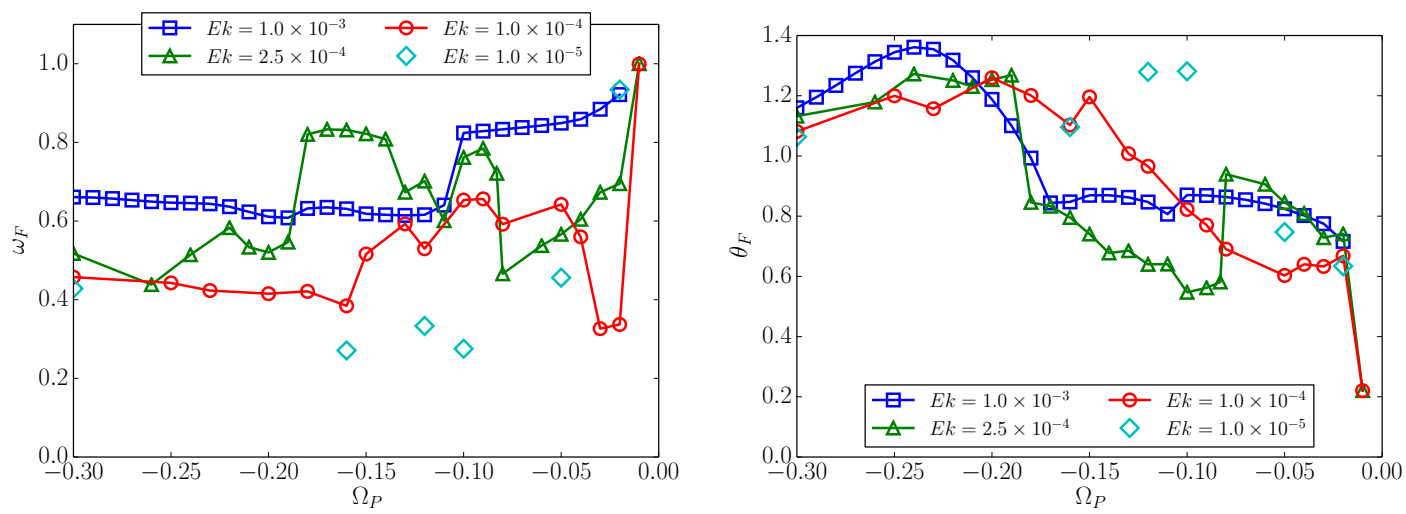

Figure 3.9: $\omega_{F}\left(\Omega_{P}\right)$ (left) and $\theta_{F}\left(\Omega_{P}\right)$ (right) with $r_{\text {end }}=0.1$ for different Ekman numbers: The transitions at $\mathrm{Ek}=2.5 \cdot 10^{-4}$ and $\mathrm{Ek}=1.0 \cdot 10^{-4}$ can be clearly seen at $\omega_{F}$ while for $\mathrm{Ek}=1.0 \cdot 10^{-3}$ only the first one appears and $\mathrm{Ek}=1.0 \cdot 10^{-5}$ shows none. On $\theta_{F}$ on the other hand, the transitions in $\mathrm{Ek}=2.5 \cdot 10^{-4}$ are notable too but not so for $\mathrm{Ek}=1.0 \cdot 10^{-4}$. At $\Omega_{P}=-0.3 \theta_{F} \approx 1.1$ for all Ekman numbers, a little above $\alpha$, so the rotation axis and the fluid axis are roughly aligned.

the center. In the equatorial plane normal to $z$ the movement of the borders in the precession system can explicitly be seen, since the fluid in the edges move with them. In the other pictured planes, this part of the motion is normal to the plane and therefor here unseen. Fig. 3.12 (left side) shows the variation of this kind of flow in the plane normal to $y$ at precession rates in the different regions characterized above. In the low region the flow keeps a single, simple rotation filling the whole space. However, in the middle region the flow separates in the bottom left corner from the wall, developing a separation vortex inside the corner. The main rotation dislocates subsequently and shifts away from the centrosymmetry it has in the low region. In the high region the flow gets more complicated, in opposite corners vortices are present, but the separation vortex separated itself from the corner, while a strong vortex rotating in the same direction as the main rotation dominates the corner. Watching the vorticity in the same plane in fig. 3.12 (right side), at $\Omega_{P}=-0.05$ it keeps a constant core rotation but adheres to the mantle geometry at the rim, being normal to the $x y$-planes and having approximately the value of mantle rotation $\omega_{D}$ at the others. At $\Omega_{P}=-0.12$ the picture still holds, but the fluid rotation aligns more to the edges until the angle to the $z$-axis grows even larger at precession rates like $\Omega_{P}=-0.21$ and the core fluid rotation is disjoint from the boundaries. The occurrence of the first separation vortex aligns with the transition 

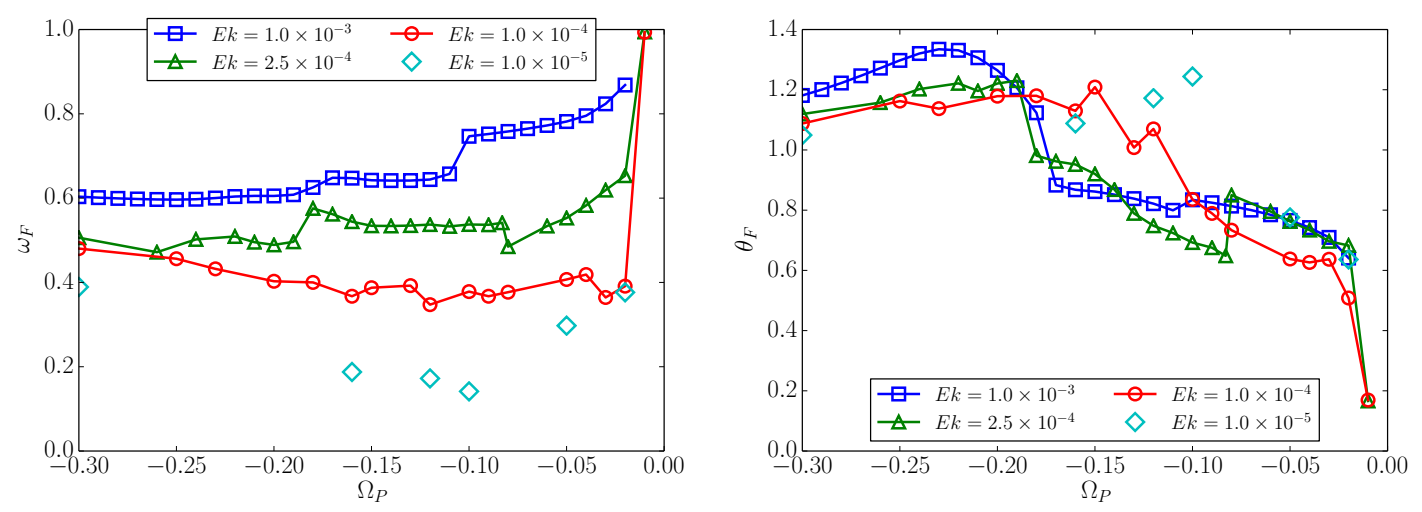

Figure 3.10: $\omega_{F}\left(\Omega_{P}\right)$ (left) and $\theta_{F}\left(\Omega_{P}\right)$ (right) with $r_{\text {end }}=0.3$ for different Ekman numbers: the same behavior is shown as in fig. 3.9, but is much more subdued.

between low and middle region in $\omega_{F}\left(\Omega_{P}\right)$ in fig. 3.12 (left side) showing a link between this and strength of fluid rotation. The cyclic rotation of a second vortex and fluid rotation detachment on the other hand can be seen in the strong growth in $\theta_{F}$ after $\Omega_{P}=-0.19$. In a three dimensional view of the vorticity like in fig. 3.13 this becomes more obvious as in the low region at $\Omega_{P}=-0.05$. We see again the adherence of the top and bottom boundaries, while the middle region shows a strong interference with a corner, generating the vortex of 3.12 (left side). At last, in the high region it develops a higher complexity with the cyclonic and anti-cyclonic vortices seen before. For lower Ekman numbers like $\mathrm{Ek}=2.5 \cdot 10^{-4}$ the systematic behaviour remains similar (s. fig. 3.14, left side): After the first simple low region up to $\Omega_{P}=-0.09$, the first separation vortices arise. However, in this case there are two vortices in opposite corners, apparently caused by the lower viscosity. The high region seems also to be more pronounced with the anticyclonic separation vortex being larger than at higher Ekman numbers. The structure of the vorticity in fig. 3.14 (right side) shows the same behavior for the high and low region, the middle region has a fluid rotation, which aligns much more with the rotation axis than at $\mathrm{Ek}=1.0 \cdot 10^{-3}$. This leads to the higher $\omega(r)$ in the center seen in fig. 3.7b and prominently differentiate the middle region from the others in fig. 3.9. For $\mathrm{Ek}=1.0 \cdot 10^{-4}$ in $\omega_{F}$ the regions are similar to that of $\mathrm{Ek}=2.5 \cdot 10^{-4}$ as they are in $\omega(r)$ and $\theta(r)$ in fig. 3.7c and of course in the streamline presentation of fig. 3.15. Neither $\omega_{F}$ nor $\theta_{F}$ show signs in fig. 3.9 for equivalent changes in flow structure at $\mathrm{Ek}=1.0 \cdot 10^{-5}$. In the streamline representation in fig. 3.16 (left side) for the precession rates $\Omega_{P}=-0.02$ and -0.05 disturbances occur, but keep confined to the corners and small. The asymmetric flow indicates a relatively turbulent field, so that the average 

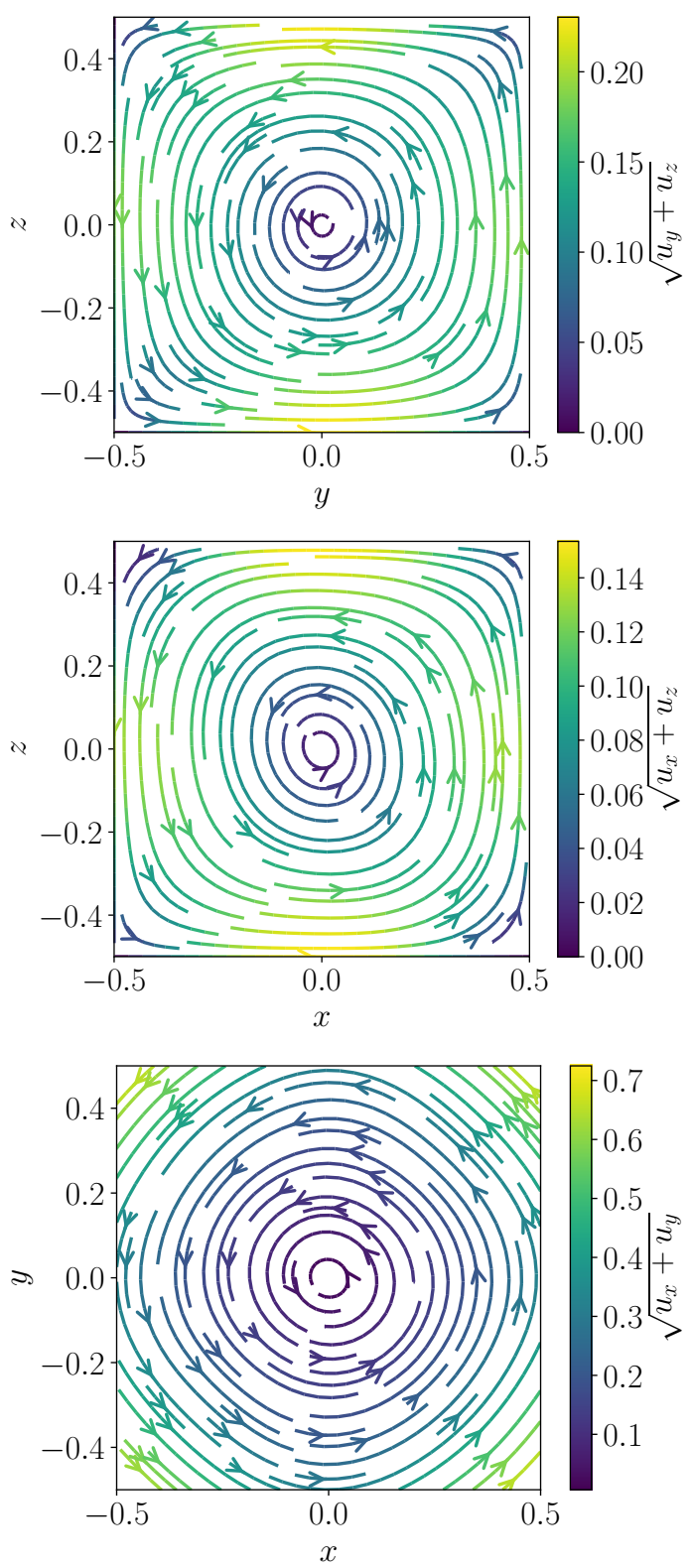

Figure 3.11: Flow in the planes with $x=0$ (top), $y=0$ (middle) and $z=0$ (bottom) for $\mathrm{Ek}=1.0 \cdot 10^{-3}$ and $\Omega_{P}=-0.05$ : The streamlines represent the velocity projected onto the respective plane while the color scale shows the actual velocity. 

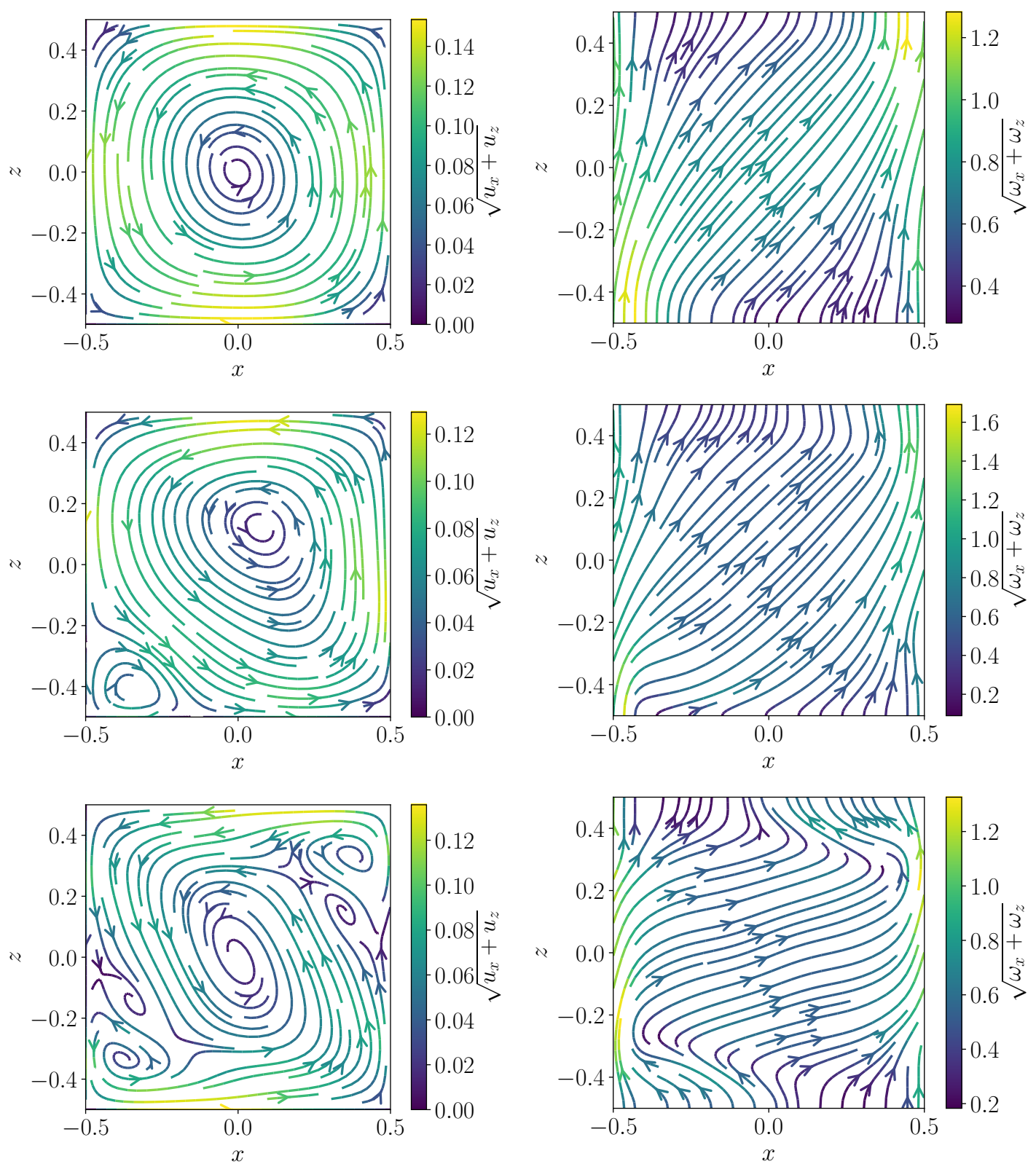

Figure 3.12: Flow (left side) and vorticity (right side) in the planes where $y=0$ for three different precession rates (from top to bottom) $\Omega_{P}=-0.05,-0.12$ and -0.21 at $\mathrm{Ek}=1.0 \cdot 10^{-3}$ corresponding to low, middle and high region. 

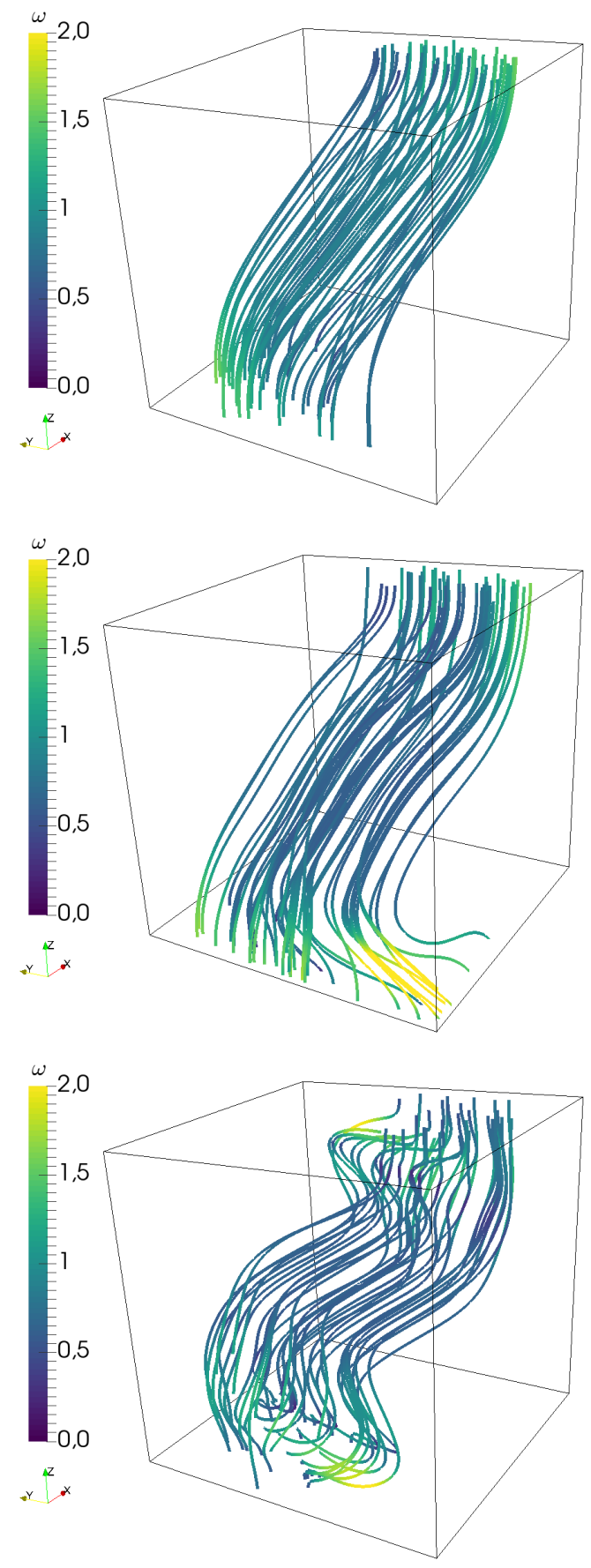

Figure 3.13: Streamlines alongside the vorticity $\omega$ at (from top to bottom) $\Omega_{P}=-0.05$, $\Omega_{P}=-0.12$ and $\Omega_{P}=-0.21$ and $\mathrm{Ek}=1.0 \cdot 10^{-3}$ show the growing complexity of the rotation at edges. 

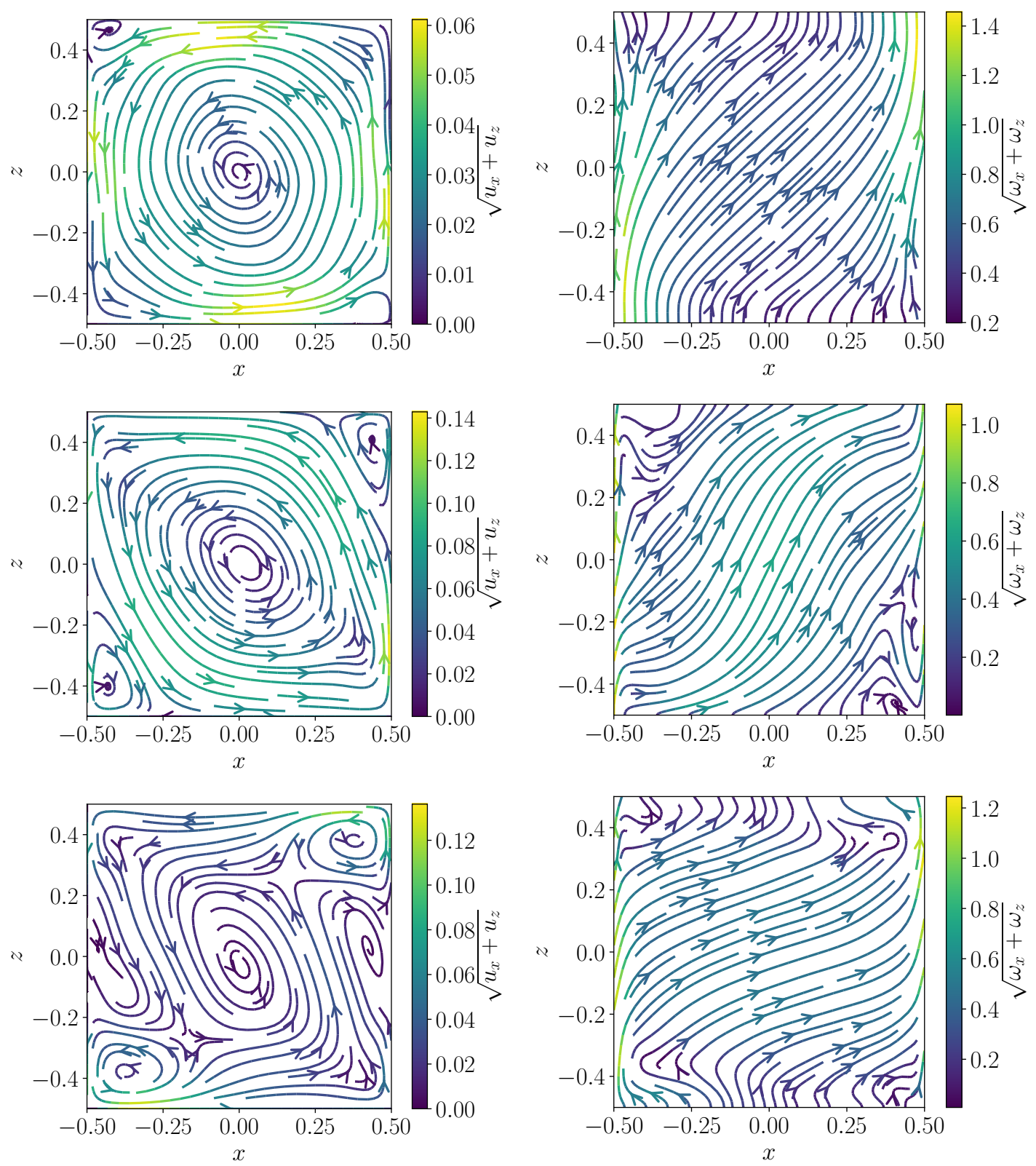

Figure 3.14: Flow (left side) and vorticity (right side) in the plane where $y=0$ for three different precession rates (from top to bottom) $\Omega_{P}=-0.05,-0.1$ and -0.2 at $\mathrm{Ek}=2.5 \cdot 10^{-4}$ corresponding to low, middle and high region. 

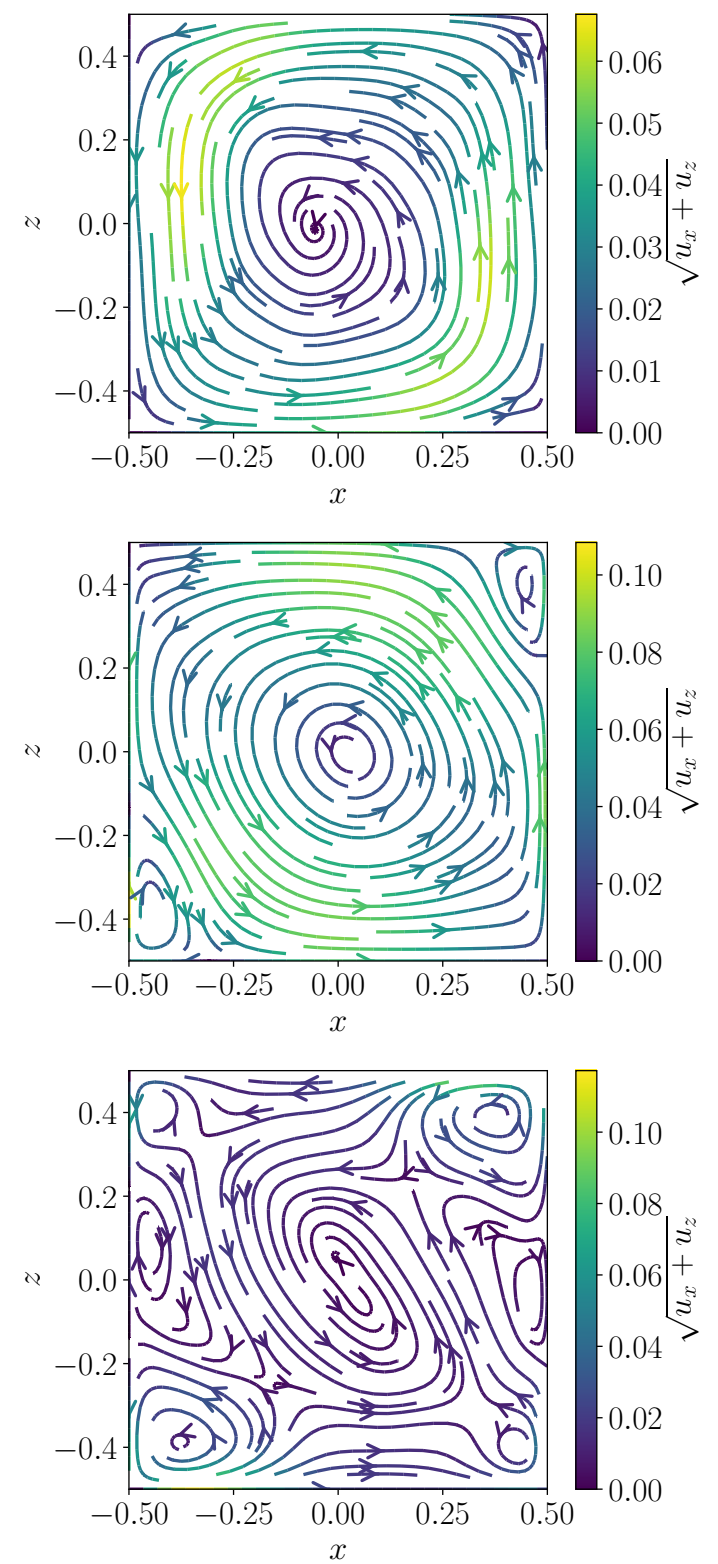

Figure 3.15: Flow in the plane where $y=0$ for three different precession rates $\Omega_{P}=$ $-0.02,-0.05$ and -0.19 at $\mathrm{Ek}=1.0 \cdot 10^{-4}$ corresponding to low, middle and high region. 
for the mean field is not sufficient to compensate for it. The vorticity in fig. 3.16 (right side) tilts as at higher Ekman numbers, but is disjointed from the boundaries.
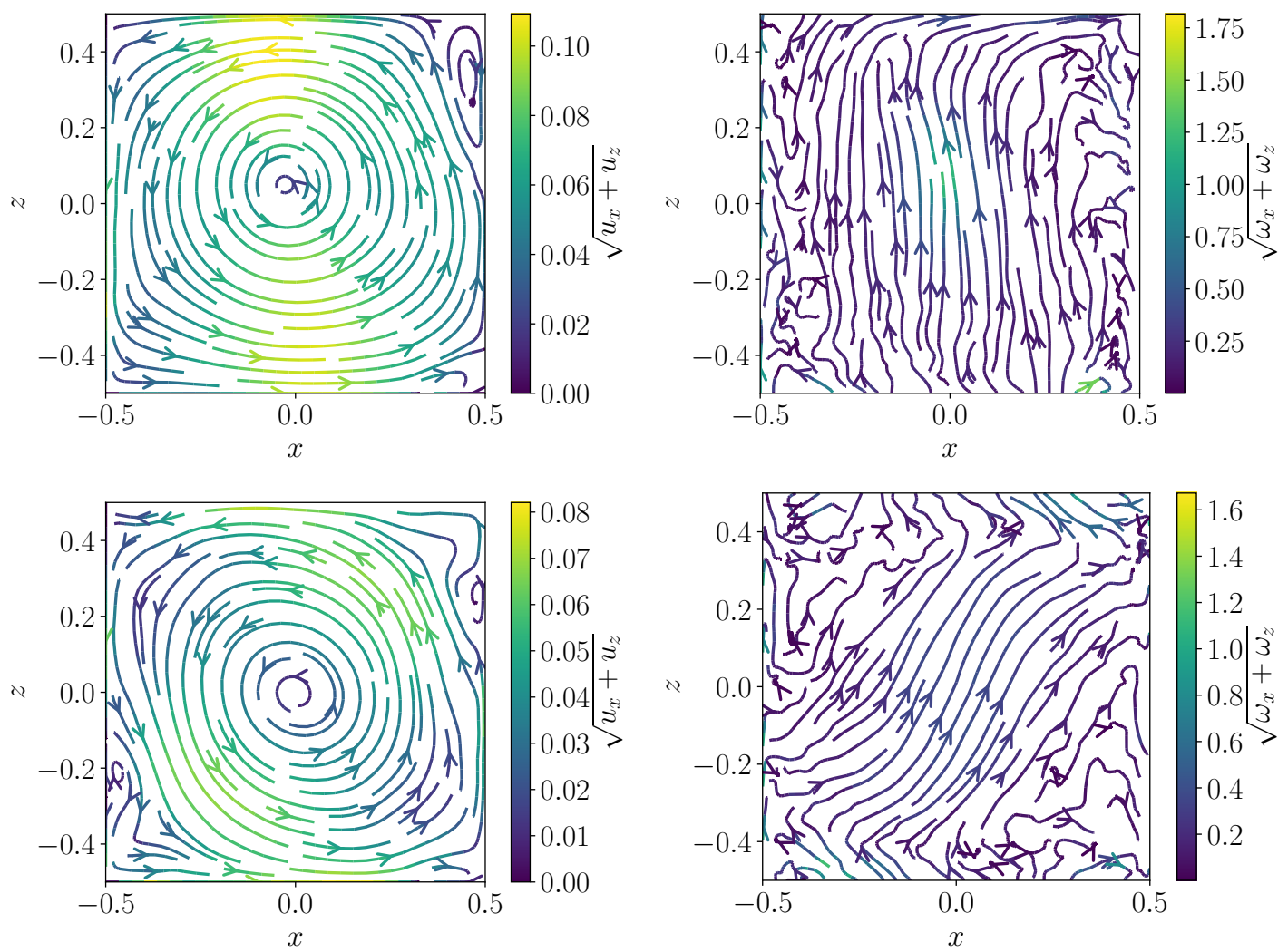

Figure 3.16: Flow (left side) and vorticity (right side) in the plane where $y=0$ for three different precession rates (from top to bottom) $\Omega_{P}=-0.02$ and -0.05 at $\mathrm{Ek}=1.0 \cdot 10^{-5}$.

\subsection{Single Vortex State}

The high peak of radial vorticity $\omega(r)$ at $r=0$ around $\mathrm{Ek}=1.0 \cdot 10^{-5}$ and $\Omega_{P}=-0.02$ in fig. $3.8 \mathrm{c}$ as well as $\omega_{F}$ in 3.9 can be attributed to a single cyclonic vortex, which emerges inside the bulk at this point. To visualize this flow it is favorable to change the reference system to the frame of reference of the fluid: The tripod $\left(x^{\prime}, y^{\prime}, z^{\prime}\right)$ will be defined, so that the $z^{\prime}$-axis aligns with the fluid axis $\omega_{F}$ as it is set in eq. 3.2. The plane perpendicular to $\boldsymbol{z}^{\prime}$ going through the origin is now the plane, in which the fluid rotates and the component $\omega_{z}^{\prime}$ shows the part of the flow which belongs to the two-dimensional rotation defined by $\boldsymbol{\omega}_{F}$. This is sketched in fig. 3.17 . 


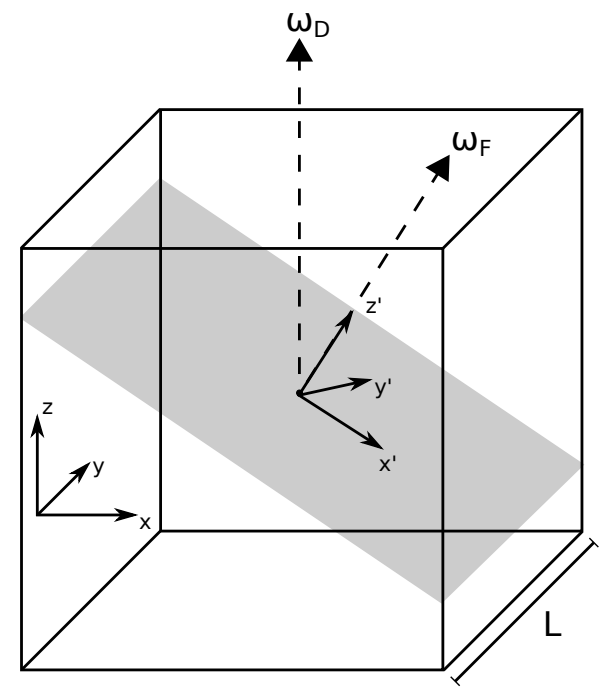

Figure 3.17: The reference system for the mantle and for the fluid are sketched here: The mantle reference system $(x, y, z)$ is fixed in respect to the boundaries with the $z$-axis alongside the rotation axis, the fluid reference system $\left(x^{\prime}, y^{\prime}, z^{\prime}\right)$ moves inside the boundaries, so that $z^{\prime}$ points alongside $\boldsymbol{\omega}_{F}$

With this done, figure 3.18 shows $\omega_{z^{\prime}}$ in the plane normal and alongside $\boldsymbol{\omega}_{F}$ : The vortex stands clearly out against the chaotic vorticity at the boundaries and spreads through the whole container with small variation in strength. At other precession rates like $\Omega_{P}=-0.04$ in fig. 3.19 this behavior vanishes and instead small scale turbulence is dominant. At higher Ek similar behavior is apparent like in fig. 3.20 for $\mathrm{Ek}=1.0 \cdot 10^{-4}$ and $\Omega_{P}=-0.04$. The vortex is not so pronounced as in fig. 3.18 and does not traverse the whole container in this distinctive tube form. As it stands, there is an evolution of vortex generation from high to low Ekman numbers.

If the vortex occurs, it is characterized by vorticity alongside the fluid axis greater than one. A more simple condition related to the rotation axis but of similar relevance is:

$$
\omega_{\text {mantle }, z}>0
$$

indicating a cyclonic rotation. To evaluate this, flow simulations are examined for a duration $T$ of 100 rotations (corresponding roughly $T \approx 600$ ) for $\mathrm{Ek} \geq 10^{-4}$ and 50 rotations for $\mathrm{Ek}<10^{-4}$ due to the higher computational effort by the necessary higher resolution at lower Ekman numbers. From this simulations the local vorticity $\omega_{\text {mantle }, z}$ is used to identify clusters of directly adjacent points fulfilling eq. 3.3. For this, all adjacent grid points of each clusters are uniquely labeled by a connected-component labeling 

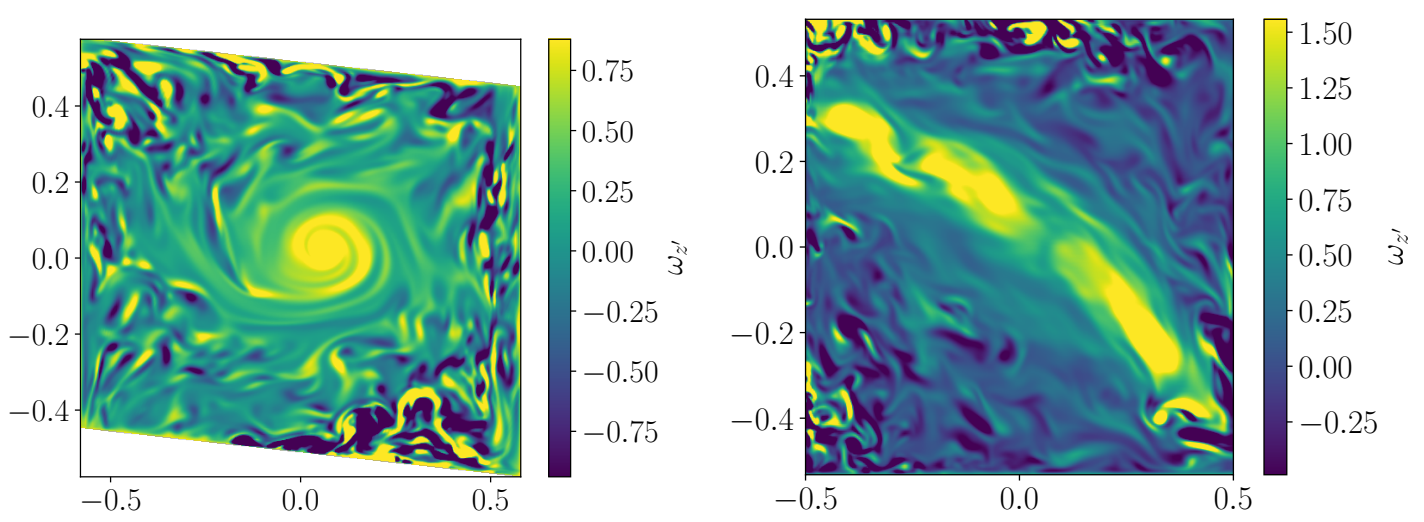

Figure 3.18: $\omega_{z^{\prime}}$ for $\mathrm{Ek}=1.0 \cdot 10^{-5}$ and $\Omega_{P}=-0.02$ in the plane normal (left) and parallel (right) to $\boldsymbol{z}^{\prime}$ through the origin.
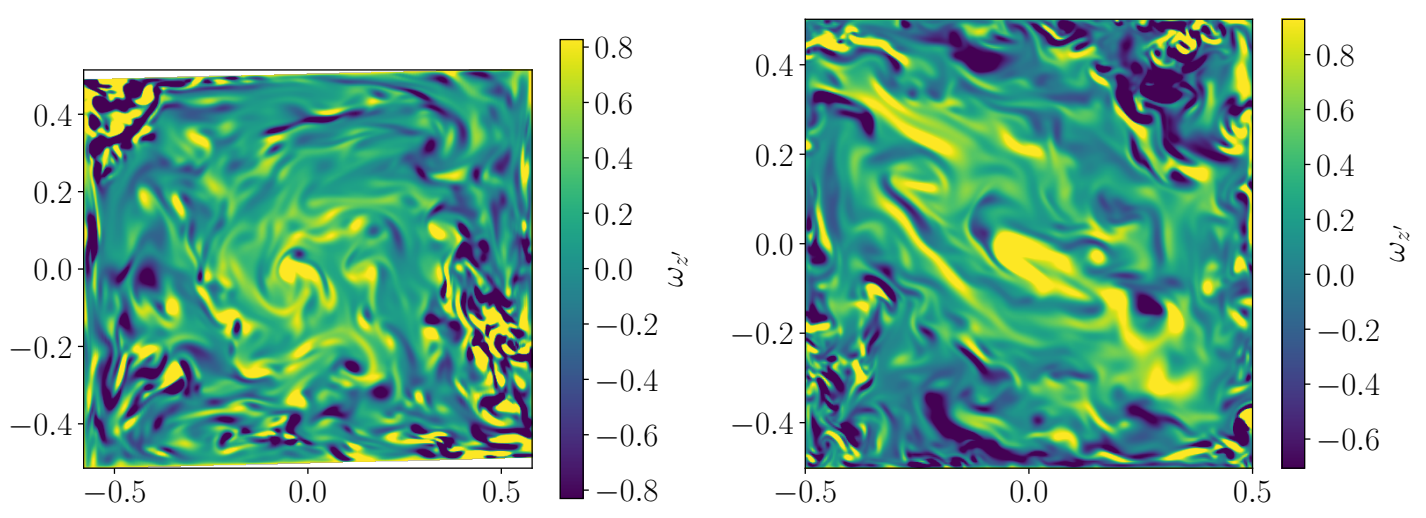

Figure 3.19: $\omega_{z^{\prime}}$ for $\mathrm{Ek}=1.0 \cdot 10^{-5}$ and $\Omega_{P}=-0.04$ in the plane normal (left) and parallel (right) to $\boldsymbol{z}^{\prime}$ through the origin.
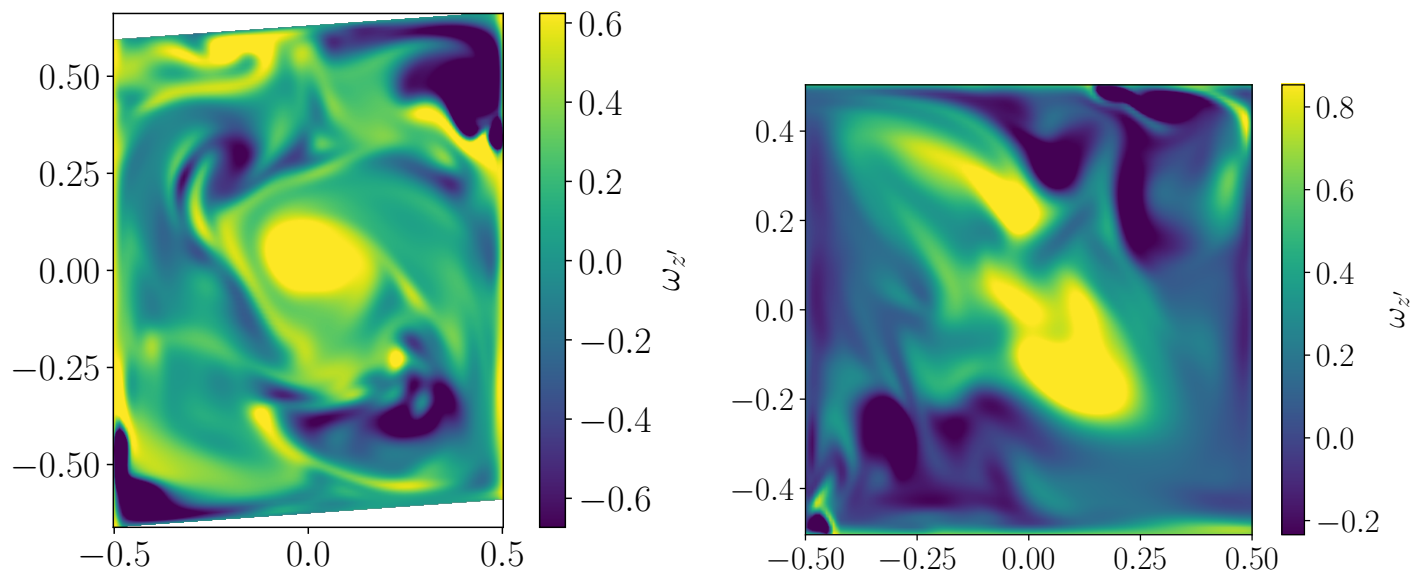

Figure 3.20: $\omega_{z^{\prime}}$ (right) for $\mathrm{Ek}=1.0 \cdot 10^{-4}$ and $\Omega_{P}=-0.1$ in the plane normal (left) and parallel (right) to $\boldsymbol{z}^{\prime}$ through the origin. 
algorithm. For a point at position $\left(x_{i}, y_{j}\right)$ the points $\left(x_{i+1}, y_{j}\right),\left(x_{i}, y_{j+1}\right),\left(x_{i-1}, y_{j}\right)$, $\left(x_{i}, y_{j-1}\right)$ are defined as adjacent and all their adjacent points, as long as they fulfill eq. 3.3. These clusters can have two different sources: the vortex on the one hand, turbulence on the other hand. Turbulence tend to be small scale and the central vortex dominates the bulk of the flow in fig 3.18 , it is usually the cluster with the largest size in the bulk.

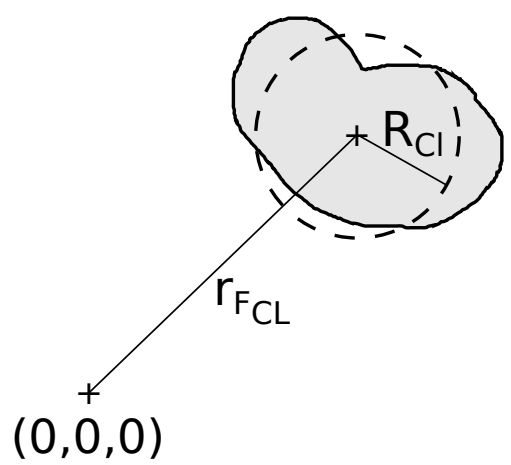

Figure 3.21: The parameters $R_{C l}$ and $r_{F_{C l}}$ illustrated: If a cluster (grey area) is reformed to a perfect circle (dashed line), $R_{C l}$ defines the radius of this circle, while $r_{F_{C l}}$ is the distance between its center and the origin.

The use of just a plane to conduct this investigation makes the size of cluster $C l$ an area of adjacent grid points with

$$
F_{C l}=\sum_{q \in C l} \Delta x_{i^{q}} \Delta y_{j^{q}}
$$

with $q \in C l$ if $\left(x_{i}^{q}, y_{j}^{q}\right)$ satisfies eq. 3.3 and is adjacent to other points in $C l . F_{C l}$ is now a closed surface of arbitrary shape on the plane, but to get a more descriptive picture, $F_{C}$ is reduced to a hypothetical radius $R_{C l}$, which would be accurate, if the area formed a perfect circle, so

$$
R_{C l}=\sqrt{\frac{F_{C l}}{\pi}} .
$$

Actually, this relation may often be true approximately for the sought-for central vortex, but is primary thought for a better understanding of the size of it. The barycenter of the surface of cluster $C l$ is defined by

$$
\boldsymbol{r}_{F_{C l}}=\frac{1}{F_{C l}} \sum_{j \in C l} \boldsymbol{r}_{j} \Delta x_{j} \Delta y_{j}
$$



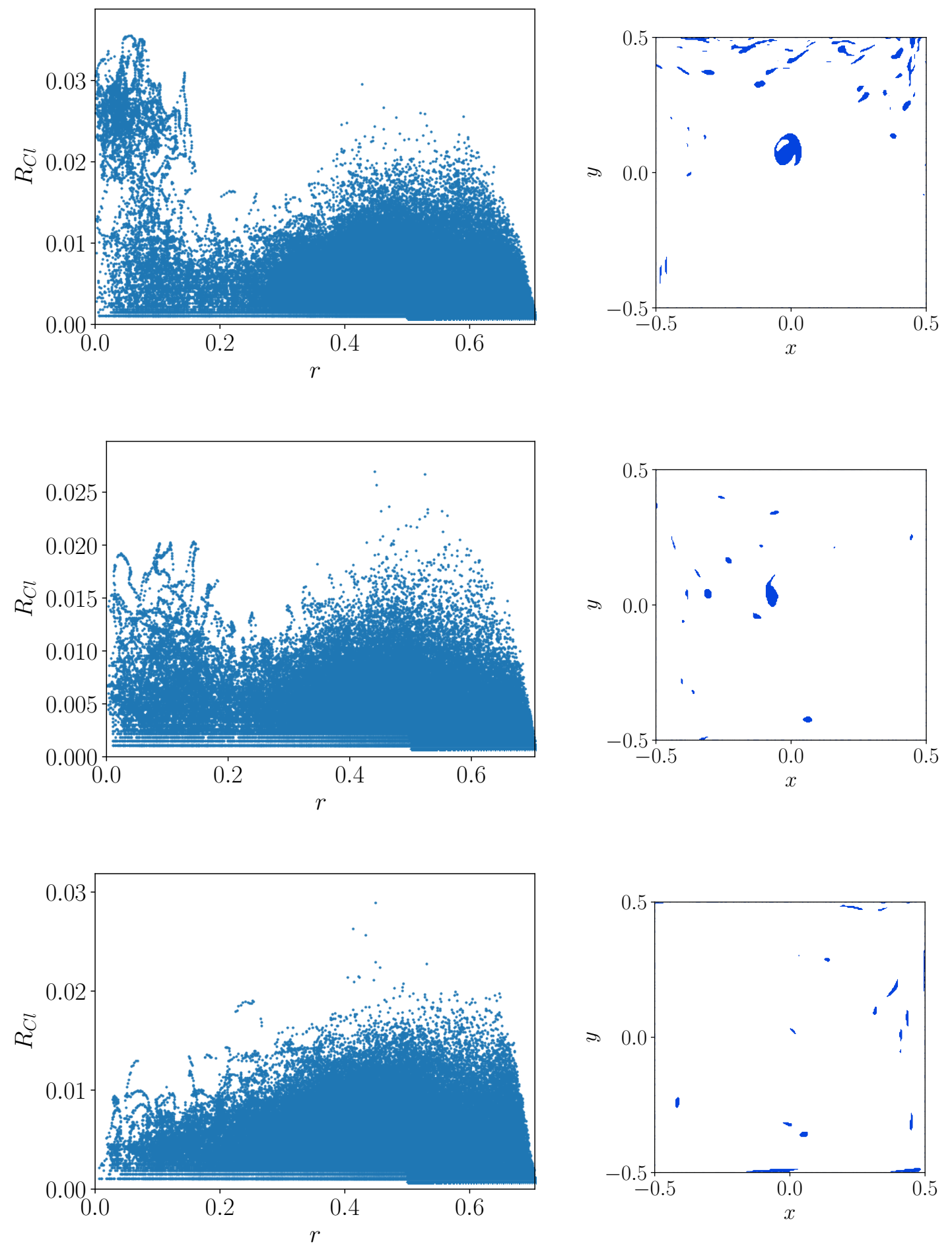

Figure 3.22: $R_{C l}\left(r_{F_{C l}}\right)$ (left side) and clusters on $z=0$-plane through the origin at a random point of time (right side) for (from top to bottom) $\mathrm{Ek}=1.0 \cdot 10^{-5}$ and $\Omega_{P}=-0.02$, Ek $=1.0 \cdot 10^{-5}$ and $\Omega_{P}=-0.03$ and $\mathrm{Ek}=1.0 \cdot 10^{-5}$ and $\Omega_{P}=-0.1$. 
The geometric relation between these parameters is sketched in fig. 3.21. The corresponding pairs $\left(R_{C l}, r_{F_{C l}}\right)$ for every cluster identified in the plane during 50 rotations are plotted in fig. 3.22 for $\mathrm{Ek}=1.0 \cdot 10^{-5}$ and $\Omega_{P}=-0.02,-0.03$ and -0.1 . For $\Omega_{P}=-0.02$ a point cloud at $R_{C l} \approx 0.025$ and $r_{F_{C l}} \approx 0.1$ marks the dominating points, which form the center of the vortex, while at $r_{F_{C l}}>0.2$ a lot of small cluster appear, very likely originating from turbulence generating effects from the boundaries reaching up to the center of the container. At $\Omega_{P}=-0.1$ there is no dominating central vortex, only the boundary turbulence remains. This becomes clearer by looking at higher Ekman numbers like in fig. 3.23: At $\mathrm{Ek}=2.5 \cdot 10^{-4}$ and $\Omega_{P}=-0.1$ the central vortex is smaller than at $\mathrm{Ek}=1.0 \cdot 10^{-5}$ but still contained to $r_{F_{C l}}<0.2$, while the boundary turbulence never reaches beyond $r_{F_{C l}}=0.3$. At $\mathrm{Ek}=3.0 \cdot 10^{-4}$ and $\Omega_{P}=-0.13$ the structure is less chaotic, the central vortex stays at $r_{F_{C l}} \approx 0.0$ and the clusters at the boundary are mostly at $r_{F_{C l}} \geq 0.5$. Since $r=0.5$ is the shortest distance of the container boundaries to the center, the boundary clusters originate from the corners of the container. As seen at the behavior at different precession rates in fig. 3.22, their occurrence does not depend on the existence of the central vortex, but is in fact part of the overall flow structure.

For low precession rates, a boundary layer with $\omega_{\text {mantle, } z}>0$ can exist, generating clusters with high $R_{C l}$ but low $r_{F_{C l}}$ as in fig. 3.24, although the cluster only exists at the boundary. When looking at the mean distance of cluster surface defined as

$$
\tilde{r}_{F_{C l}}=\frac{1}{F_{C l}} \sum_{j \in C l}\left|\boldsymbol{r}_{j}\right| \Delta x_{j} \Delta y_{j}
$$

the cluster at small $r_{F_{C l}}$ reorganizes at $\tilde{r}_{F_{C l}} \approx 0.5$. With knowledge of the structure in figs. 3.22 and $3.23,3.24$, one can identify the central vortex by taking the largest cluster with $\tilde{r}_{F_{C l}}<0.2$. The mean value of the size $R_{C l}$ of the vortex, $R_{\text {vortex }}$, is plotted in fig. 3.25 for different values of $\mathrm{Ek}$ and $\Omega_{P}$. For $\mathrm{Ek}=2.5 \cdot 10^{-4}, R_{\text {vortex }}$ has considerable amounts only in the region $\Omega_{P}=-0.13$ to -0.09 . At $\mathrm{Ek}=1.0 \cdot 10^{-4}$ the lower limit of this behavior is at $\Omega_{P}=-0.04$, while for $\Omega_{P}<-0.13$ a small region around $R_{\text {vortex }} \approx 0.004$ exists, produced by the boundary turbulence in the absence of any dominating central vortex like at $\mathrm{Ek}=1.0 \cdot 10^{-5}, \Omega_{P}=-0.1$ in fig. 3.22. This is again visible for $\mathrm{Ek}=5.0 \cdot 10^{-5}$ for all $\Omega_{P}<-0.07$ and for $\mathrm{Ek}=1.0 \cdot 10^{-5}$ for all $\Omega_{P}<-0.04$ at the parameters simulated.

The central vortex region migrates to smaller precession rates for decreasing Ek and simultaneously the maximum of $R_{\text {vortex }}$ increases to 0.025 at the lowest simulated Ek, $1.0 \cdot 10^{-5}$. The motion of this cluster's barycenter $\boldsymbol{r}_{F_{C l}}$ is visualized in fig. 3.26 for 

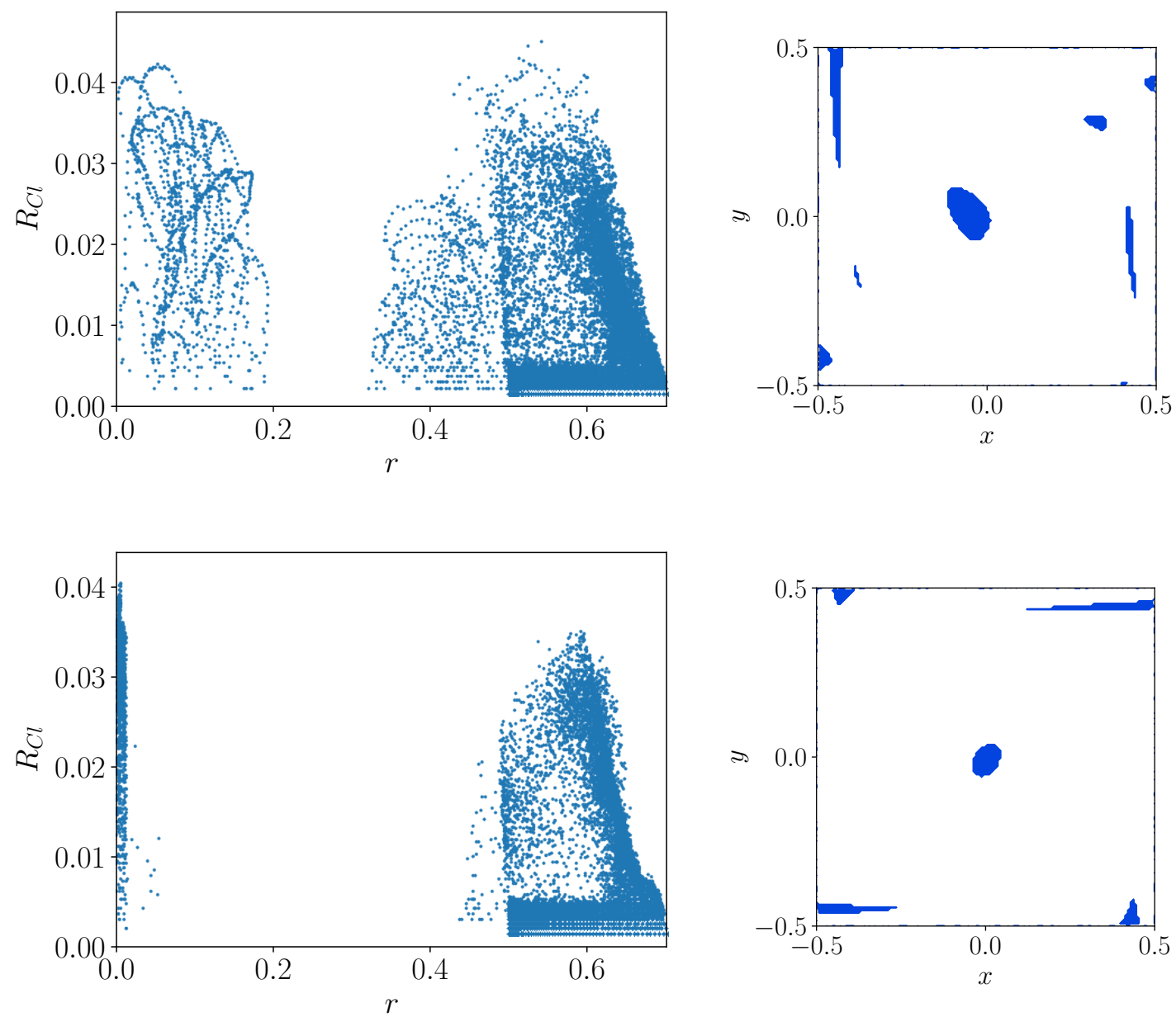

Figure 3.23: $R_{C l}\left(r_{F_{C l}}\right)$ (left side) and clusters on $z=0$-plane through the origin at a random point of time (right side) for $\mathrm{Ek}=2.5 \cdot 10^{-4}$ and $\Omega_{P}=-0.1$ (top), $\mathrm{Ek}=3.0 \cdot 10^{-4}$ and $\Omega_{P}=-0.13$ (bottom). 

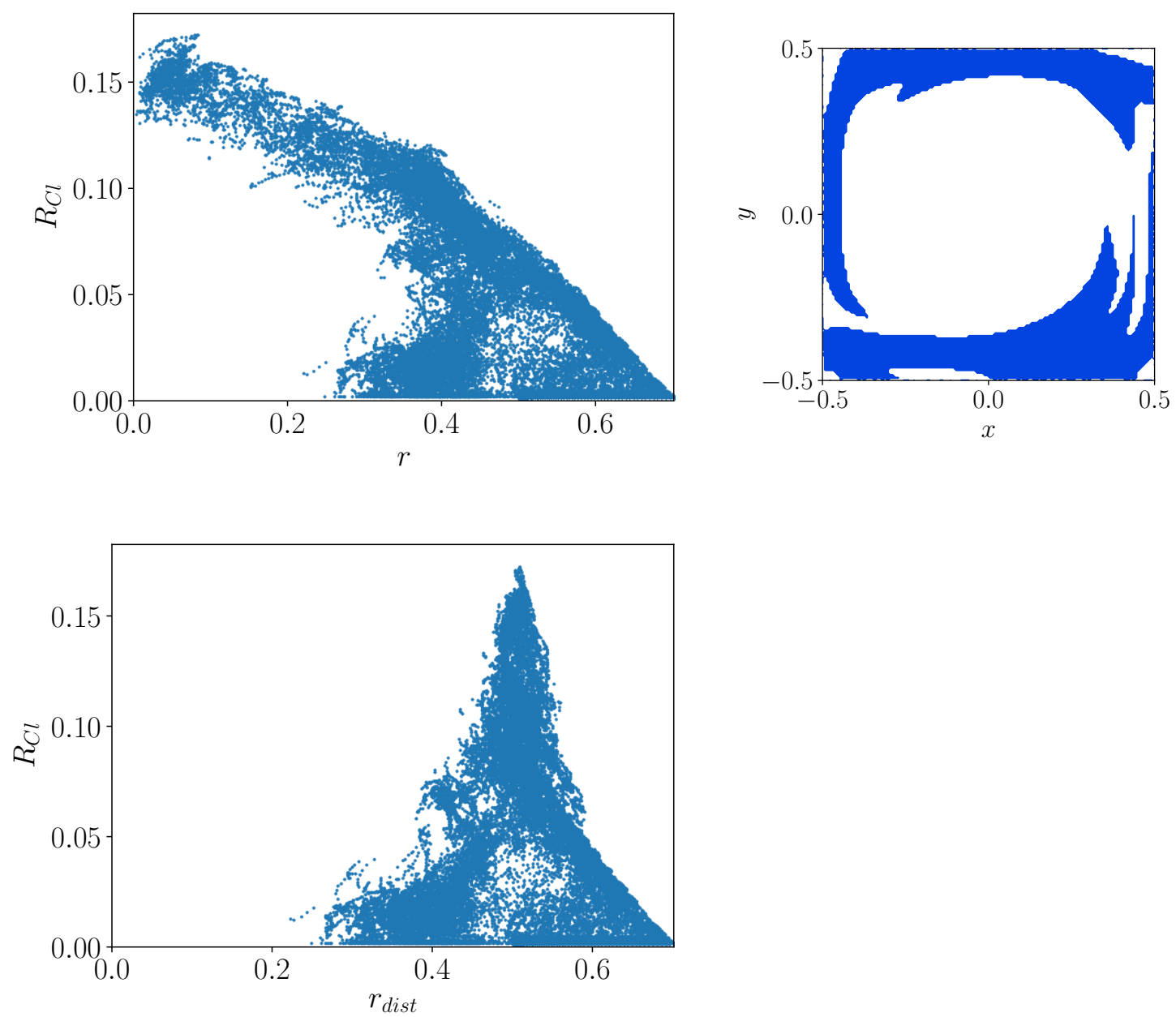

Figure 3.24: $R_{C l}\left(r_{F_{C l}}\right)$ (top, left side) and clusters on $z=0$-plane through the origin at a random point of time (top, right side) for $\mathrm{Ek}=1.0 \cdot 10^{-4}$ and $\Omega_{P}=-0.03$ $R_{C l}\left(\tilde{r}_{F_{C l}}\right)$ (bottom) for the same parameters. 
16 rotations in the precession reference system. For the whole duration, the vortex existed always and with relatively steady size. But depending on the Ekman number, the variation in size over time can be much larger. In fig. 3.27 the size $R_{C l}$ are plotted for three different $\mathrm{Ek}$ and $\Omega_{P}: \mathrm{Ek}=3.0 \cdot 10^{-4}$ and $\Omega_{P}=-0.13, \mathrm{Ek}=5.0 \cdot 10^{-5}$ and $\Omega_{P}=-0.04$ and $\mathrm{Ek}=1.0 \cdot 10^{-5}$ and $\Omega_{P}=-0.02$. For all cases, the maxima of $R_{\text {vortex }}$ reach values roughly around 0.025 , for $\mathrm{Ek}=3.0 \cdot 10^{-4}$, however, the vortex only appears periodically for a small duration. At $\mathrm{Ek}=5.0 \cdot 10^{-5}$ it exists for longer time spans, but its variation in size is more chaotically. At $\mathrm{Ek}=1.0 \cdot 10^{-5}$ finally, it is preserved every time. The ratio of time with existing central vortex $t_{\text {vortex }}$ against the whole duration $T$ is presented in 3.28. For $t_{\text {vortex }}$ the time was taken, when $R_{\text {vortex }}(t)>0.01$. This limit was chosen to exclude the impact of turbulence at $r<0.2$ as described before. Again, we see a similar behavior as in fig. 3.25 with increasing $\frac{t_{\text {vortex }}}{T}$ for decreasing Ek. Not above $\mathrm{Ek}=1.0 \cdot 10^{-5}$ a central vortex becomes permanent. This is mainly caused by a growing lifespan: Lasting only for a few rotations for high Ek (see fig. 3.29), the vortex lifespan $\tau_{\text {vortex }}$ rises above 30 rotations at $\mathrm{Ek}=5.0 \cdot 10^{-5}$ and is of the charts for $\mathrm{Ek}=1.0 \cdot 10^{-5}$ and $\Omega_{P}=-0.02$ because of the permanent occurrence. But even at this point, the vortex shows some degradation into vortices of smaller scales. Over the observed time the single vortex splits into separate smaller vortices as in 3.30 without $R_{\text {vortex }}$ falling below 0.01 for the biggest one. This is apparent when observing the number of clusters identified within $r<0.2$ in fig. 3.31. The number grows at two occasions in the observed time span at $t \approx 60$ and 200 with roughly 22 rotations between them.

In fig. 3.32, all measured parameter values of Ek and $\Omega_{P}$ are mapped. The dots define runs with $R_{\text {vortex }}>0.01$ after a simulated duration of $T$, while crosses mark the opposite case. As one can see, the vortex state starts to broaden over the possible precession rates from $\mathrm{Ek}=3.0 \cdot 10^{-4}$ with only one run at $\Omega_{P}=-0.13$ fulfilling $R_{\text {vortex }}>0.01$ up to $\mathrm{Ek}=1.0 \cdot 10^{-4}$ with the vortex state ranging from $\Omega_{P}=-0.13$ to -0.04 as already noticed in fig. 3.25. Lowering the Ekman number impedes the formation of the vortex again, as the range of the vortex state decreases again.

\subsection{Axisymmetric modes}

Findings in a precessing experiment in a cylindrical containment and subsequent numeric simulations [Giesecke et al., 2018] emphasize the possible use of axisymmetric modes for dynamo action. Unfortunately, the understanding of inertial modes in Cartesian 


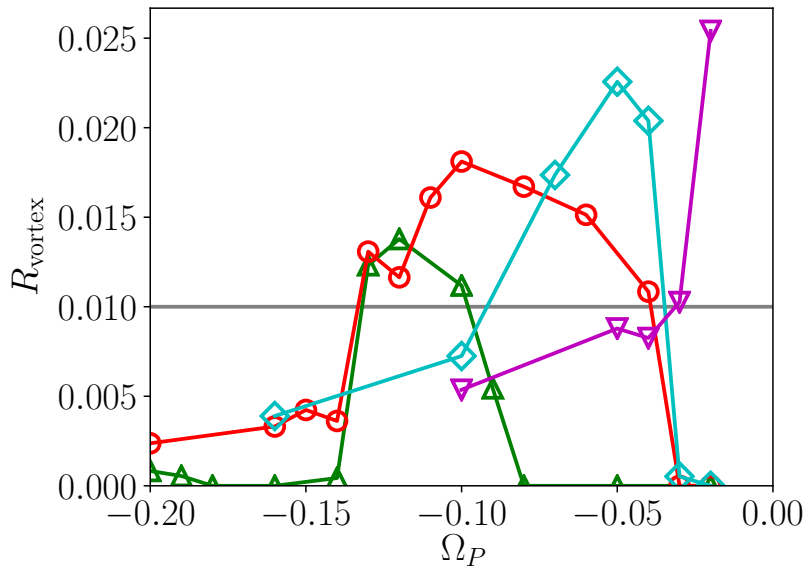

Figure 3.25: $R_{\text {vortex }}$ for $\mathrm{Ek}=2.5 \cdot 10^{-4}$ (upward triangles), $\mathrm{Ek}=1.0 \cdot 10^{-4}$ (circles), $\mathrm{Ek}=5.0 \cdot 10^{-5}$ (diamonds) and $\mathrm{Ek}=1.0 \cdot 10^{-5}$ (downward triangles).

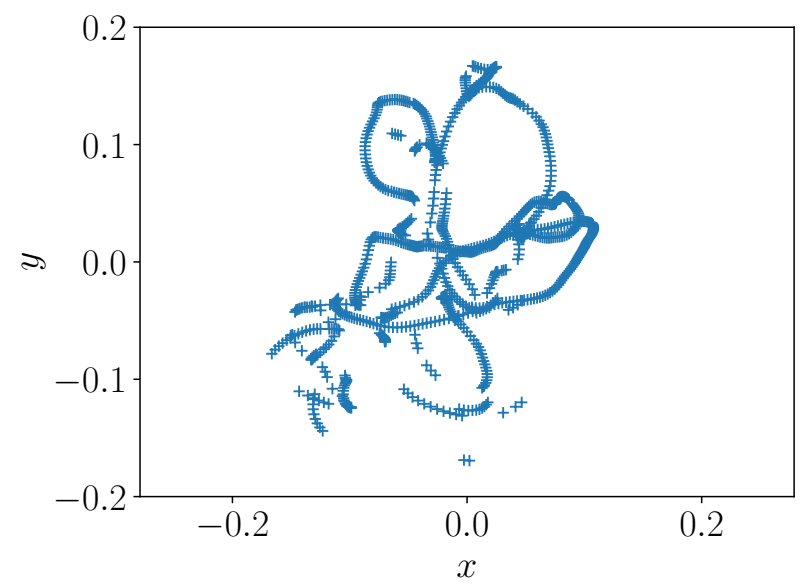

Figure 3.26: Position of the center of the central vortex at $\mathrm{Ek}=1.0 \cdot 10^{-5}$ and $\Omega_{P}=$ -0.02 for 16 rotations. 


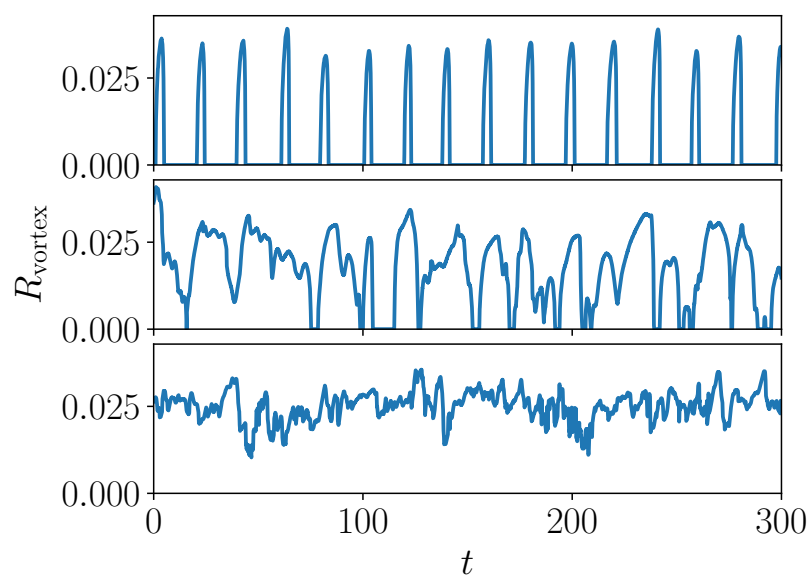

Figure 3.27: Time dependent variation of $R_{\text {vortex }}(t)$ for $\mathrm{Ek}=3.0 \cdot 10^{-4} \Omega_{P}=-0.13$ (top), $\mathrm{Ek}=5.0 \cdot 10^{-5} \Omega_{P}=-0.04$ (middle) and $\mathrm{Ek}=1.0 \cdot 10^{-5} \Omega_{P}=-0.02$ (bottom): The regular appearance of $\omega>0$ vortices at $\mathrm{Ek}=3.0 \cdot 10^{-4}$ becomes chaotic at lower Ekman numbers but are maintained for a longer time until at $\mathrm{Ek}=1.0 \cdot 10^{-5}$ they stay permanent at specific precession rates as $\Omega_{P}=-0.02$.

geometries is low with no existing analytical solution [Maas, 2003]. But in analogy to axisymmetric boundaries one can assume the number of simple dominant modes if present. This especially applies to axisymmetric modes, which will be determined as modes with azimuthal wave number $m=0$. For this cylindrical coordinates $(r, \phi, z)$ can give a better understanding of these properties, even with the restriction by the Cartesian boundaries. In this system, the boundaries enforce an azimuthal mode with wave number $m=4$ on the flow in $u_{r}$ and $u_{\phi}$, as seen in fig. 3.33. On the other hand, $u_{z}$ shows no sign of a similar influence outside the trivial confinement. Nevertheless, these coordinates open the possibility of different methods for analyzing the data.

Filtering the trivial azimuthal mode $m=0$ is an easy task even without knowledge of the exact nature of modes in the flow. Similar to the calculation of the radial vorticity distribution in eq. 3.1 the average of velocity is taken by integrating over a cylindrical shells $V^{\prime}(r, z)$ with thickness $\Delta r=\frac{1}{\min \left(N_{X}, N_{Y}\right)}$ and $\Delta z=\frac{1}{N_{Z}}$ leading to

$$
\boldsymbol{u}_{m=0}=\langle\boldsymbol{u}\rangle_{\phi}(t, r, z)=\frac{1}{V^{\prime}(r, z)} \int_{V^{\prime}(r, z)} \boldsymbol{u}(t, r, \phi, z) \mathrm{d} V .
$$

$\boldsymbol{u}_{0}$ only shows the radial mean of the velocity, hence other modes like the enforced $m=4$ 


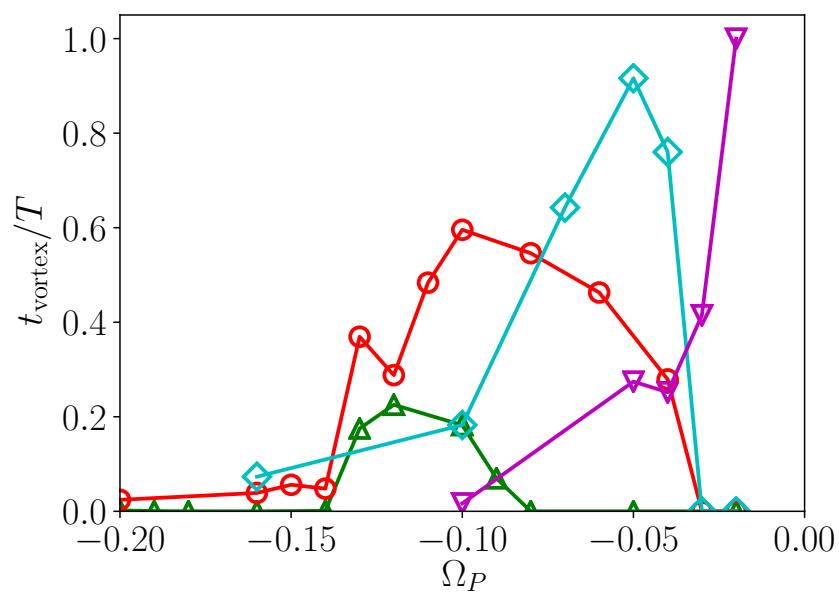

Figure 3.28: Relative time $\frac{t_{\text {vortex }}}{T}$ the vortex fulfills $R_{\text {vortex }}>0.01$ for Ek $=2.5 \cdot 10^{-4}$ (upward triangles), $\mathrm{Ek}=1.0 \cdot 10^{-4}$ (circles), $\mathrm{Ek}=5.0 \cdot 10^{-5}$ (diamonds) and $\mathrm{Ek}=1.0 \cdot 10^{-5}$ (downward triangles)

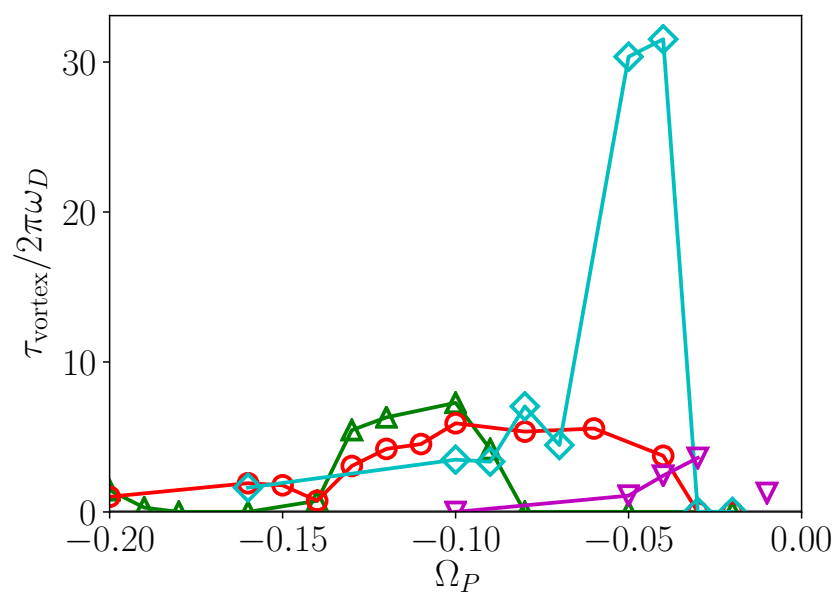

Figure 3.29: Median of vortex lifespan $\tau_{\text {vortex }}$ under condition $R_{\text {vortex }}(t)>0.01$ for Ek $=$ $2.5 \cdot 10^{-4}$ (upward triangles), $\mathrm{Ek}=1.0 \cdot 10^{-4}$ (circles), $\mathrm{Ek}=5.0 \cdot 10^{-5}$ (diamonds) and $\mathrm{Ek}=1.0 \cdot 10^{-5}$ (downward triangles) does extend radically for $\mathrm{Ek}<10^{-4}$. At $\Omega_{P}=-0.02$ and $\mathrm{Ek}=1.0 \cdot 10^{-5}$ the condition is always satisfied, so $\tau_{\text {vortex }} \rightarrow \infty$. 

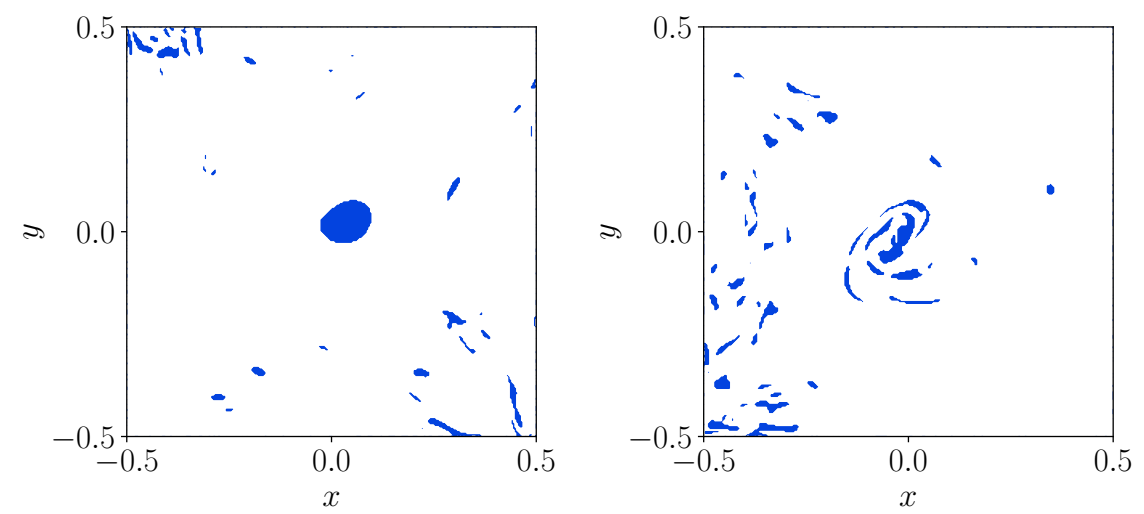

Figure 3.30: Identified clusters for the whole equatorial plane for $\mathrm{Ek}=1.0 \cdot 10^{-5}$ and $\Omega_{P}=-0.02$ at $t=100.0$ (left) and $t=203.6$ (right) show the degradation of the single vortex into smaller clusters.

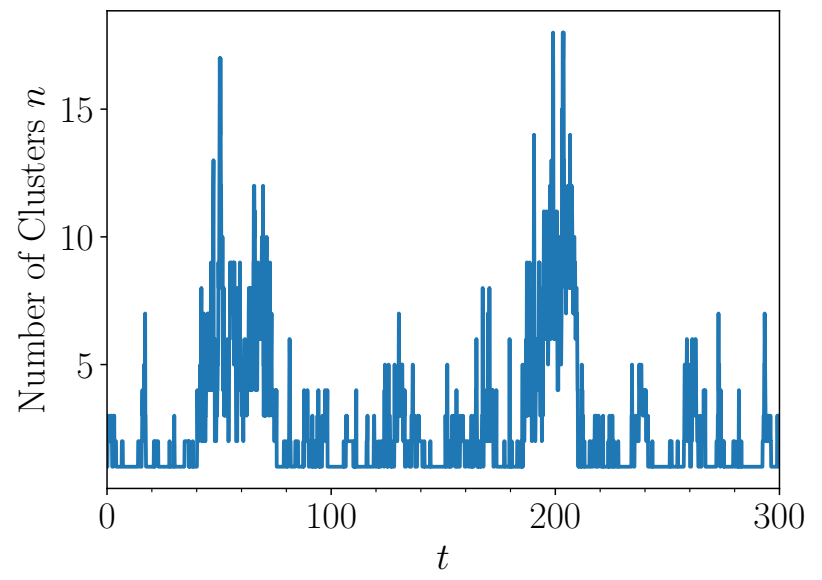

Figure 3.31: Number of clusters at one time step identified with $\omega_{z}(r<0.2)>0$ for $\mathrm{Ek}=1.0 \cdot 10^{-5}$ and $\Omega_{P}=-0.02$ often is equal or less than three, only to exceed 10 clusters at one point of time at $t \approx 60$ and 200 . 


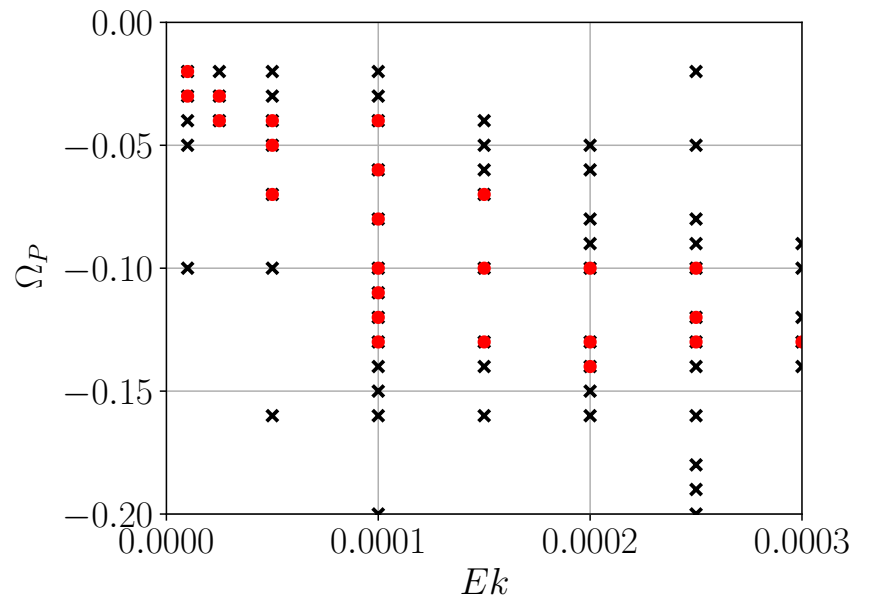

Figure 3.32: Map of all runs made for investigating vortex state: dots mark runs with $R_{\text {vortex }}>0.01$, crosses the remaining runs.

or the spin-over-mode $m=1$ are not be seen in this visualization. As an example the different components $u_{0, z}, u_{0, r}$ and $u_{0, \phi}$ for $\mathrm{Ek}=2.5 \cdot 10^{-4}, \Omega_{P}=-0.16$ are shown in 3.34. Here $u_{0, \phi}$ is dominated by a flow in positive direction. This one is constant in $z$-direction and constitutes an azimuthal circulation resembling a geostrophic mode and - since the velocity is taken in the mantle reference frame - slowing down the solid body rotation. In $u_{0, z}$ one notices the symmetries inside this flow: In $r$ the outer part flows in the opposite direction like in the inner part, apparently compensating the mass difference. Of course, this is not bound to the simple two-parted flow, but it is very likely preferred to more complicated ones. Fittingly, $u_{0, r}$ add to this picture, providing the horizontal movement compensating for the vertical $u_{0, z}$ flow. If put together in a streamline visualization only recognizing these two components, they form two counterrotating rolls like depicted in 3.35 , symmetric to the equatorial plane. This double roll features a strong inward flow at the equatorial plane and an outward flow at top and bottom. Similar to the properties of eq. 3.1 , at $r=0.5$ the cylindrical coordinates clip with the boundaries so that $\boldsymbol{u}_{0}(r>0.5)$ does not present a whole cylindrical shell anymore but a partition of the edges of the cube. This can be visualized by only examining $u_{z}(z)+u_{z}(-z)$. Here, the forced spinover-mode is removed by symmetry arguments discussed further on, but the symmetric double roll is maintained: In fig. 3.36 planes at $z=0.3$ and $y=0.0$ were drawn at $\mathrm{Ek}=2.5 \cdot 10^{-4}$. The flow orientates along the borders and shows additionally higher order structures like a visible $m=4$. To quantify the motion of velocity components for 

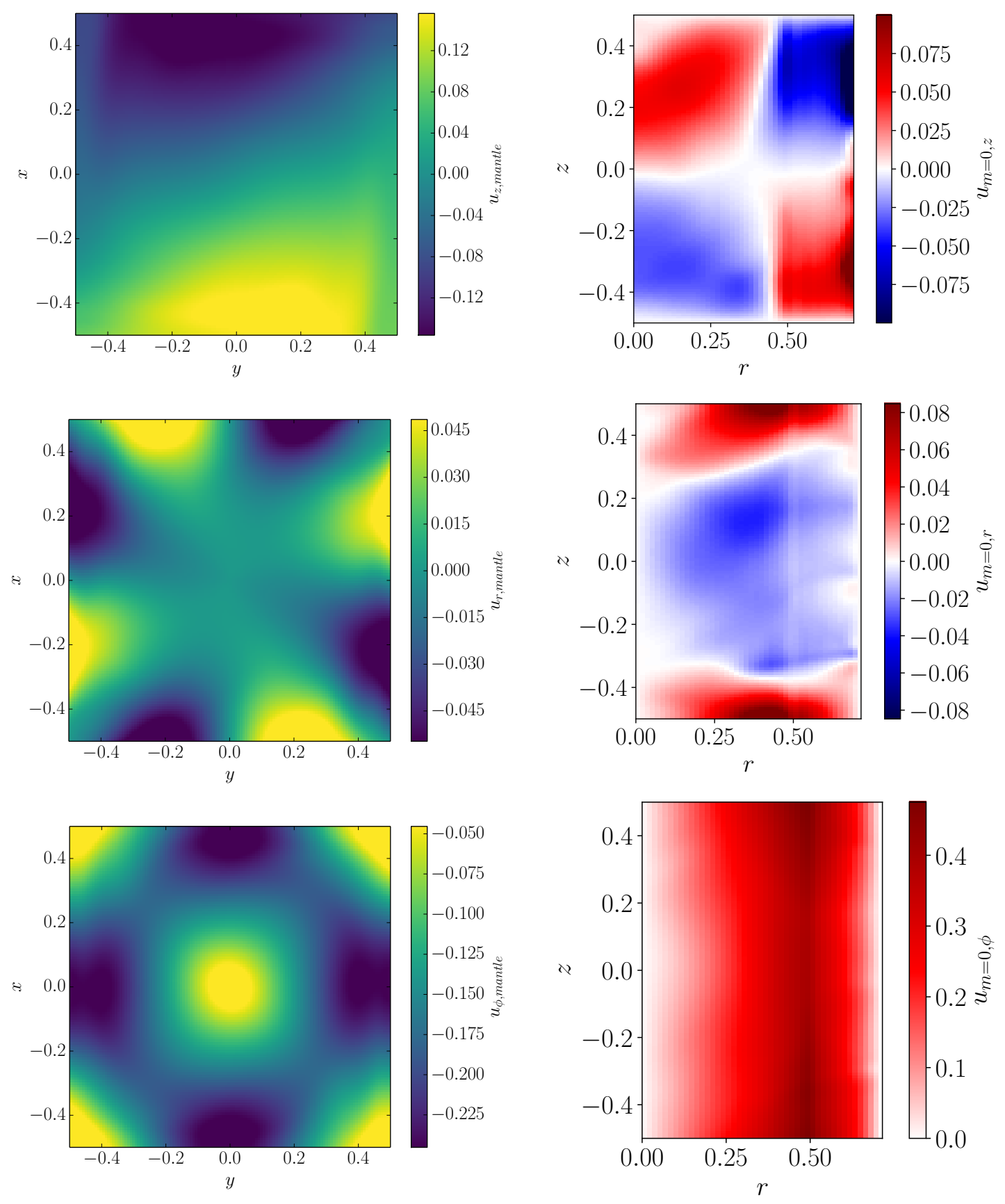

Figure 3.33: The cubic boundaries enforce a Figure 3.34: For Ek $=2.5 \cdot 10^{-4}, \Omega_{P}=$ mode on the flow in the components $u_{r}(z=0)$ and $u_{\phi}(z=0)$ $-0.16 u_{z}(r, z)$ (top) and $u_{r}(r, z)$ (middle) exhibits a (here in the mean field at $\mathrm{Ek}=$ $\left.2.5 \cdot 10^{-4} ; \Omega_{P}=-0.16\right)$, while clear visible mode in $z$ and $r$ symmetric to the plane the $u_{z}(z=0)$-component is dominated by the $m=1$-spin-overmode enforced by the precession. $z=0$, while $u_{\phi}(r, z)$ is dominated by a negative motion homogeneous in $z$-direction. 


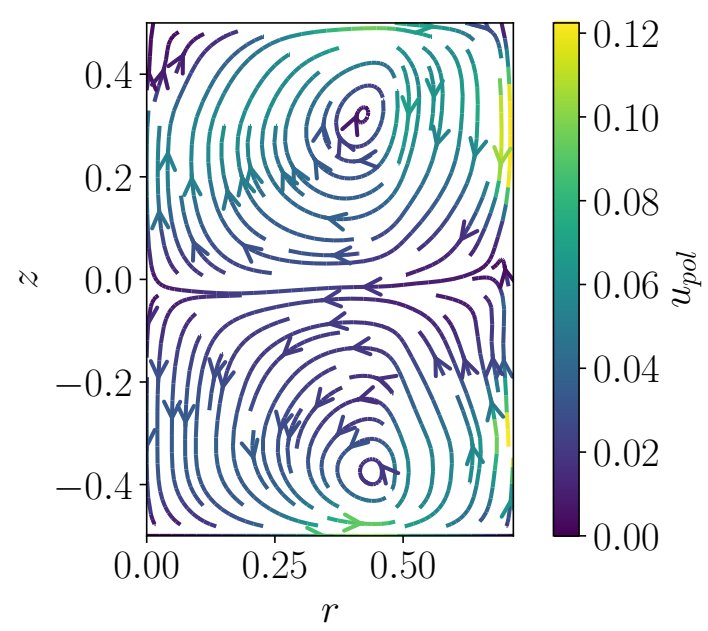

Figure 3.35: The flow in the $r$ - $z$-plane at $\mathrm{Ek}=2.5 \cdot 10^{-4}, \Omega_{P}=-0.16$ forms to a double roll with counterrotating rolls.

varying parameters the total energy of this mode was calculated by

$$
E_{m=0, i}=\frac{\iint 0.5 u_{0, i}^{2} V^{\prime}(r, z) \mathrm{drd} z}{\iint V^{\prime}(r, z) \mathrm{drdz}}
$$

with $i \in\{r, z, \phi\}$. To assess this in relation to the spin-over mode, the energy bound inside all other modes is calculated analogously to eq. 3.8 by

$$
E_{m \neq 0, i}=\frac{\iint 0.5\left\langle\left(u_{i}-u_{0, i}\right)^{2}\right\rangle_{\phi}(r, z)^{2} V^{\prime}(r, z) \mathrm{drdz}}{\iint V^{\prime}(r, z) \mathrm{drdz}} .
$$

Since it is to assume that the spin-over mode $m=1$ is the dominant one, $E_{m \neq 0, i}$ should be an approximate measure for it, especially for $E_{m \neq 0, z}$, where no $m=4$ is forced. The ratio of mean value of these two parameters, $E_{m=0, z} / E_{m \neq 0, z}$, gives fig. 3.37 (continuous lines) depicting it for different Ekman numbers and varying $\Omega_{P}$. At first one notices that not for all $\Omega_{P}$ there is a non-zero part of $E_{m=0, z} / E_{m \neq 0, z}$, and for all Ekman numbers other than $\mathrm{Ek}=1.0 \cdot 10^{-5}$ they coincide with the structural changes described in section 3.1. For $\mathrm{Ek}=2.5 \cdot 10^{-4} E_{m=0, z} / E_{m \neq 0, z}$ becomes non-zero between $\Omega_{P}=-0.17$ and -0.09 , where fig. 3.34 was taken, with a discrete rise in value and a maximum at $\Omega_{P}=-0.14$. Acknowledging the symmetry of the double roll to the $x$-y-plane (and in the same vein the antisymmetry) is helpful: The double roll is invariant under the symmetry operation

$$
(r, z) \rightarrow(r,-z)
$$

To describe axisymmetric flows forming the double roll $\boldsymbol{u}_{m=0, s}=\left(u_{m=0, z, s}, u_{m=0, r, s}\right)$ is 

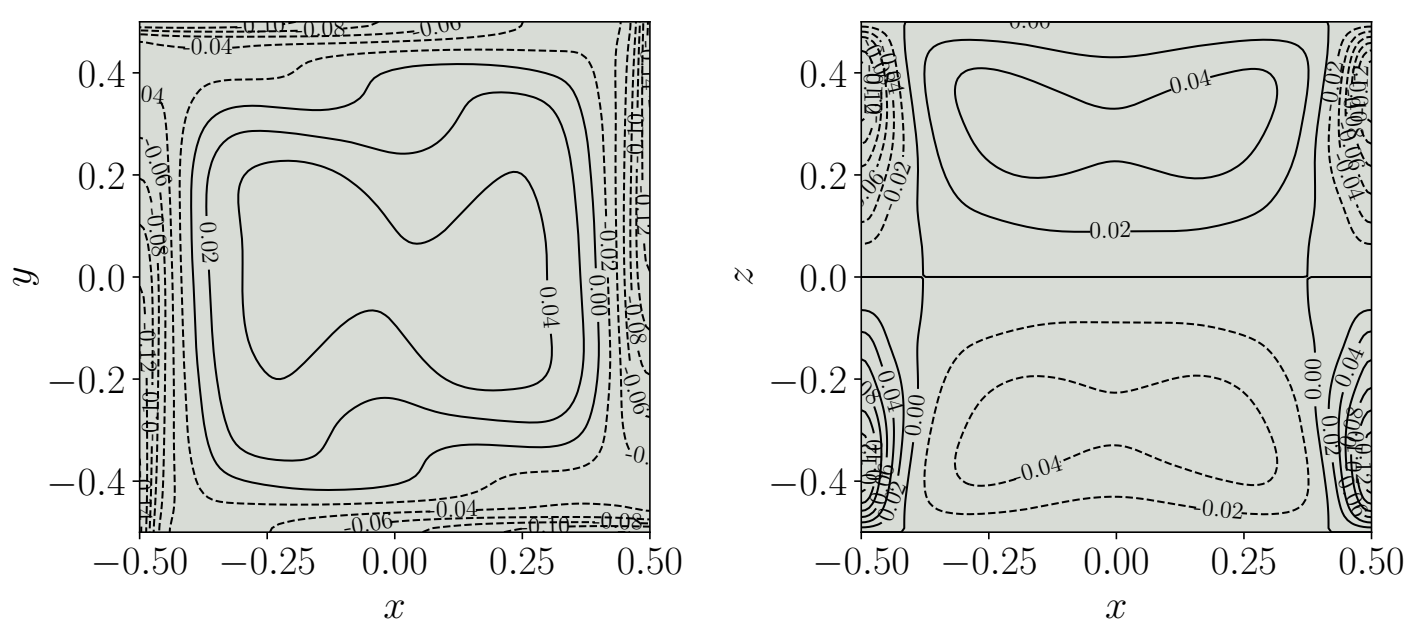

Figure 3.36: Contour plots for $u_{z}(z)+u_{z}(-z)$ in planes with $z=0.3$ and $y=0.0$ at $\mathrm{Ek}=2.5 \cdot 10^{-4}$ and $\Omega_{P}=-0.16$ show the actual composition which lead to the form of the $m=0$ if taken the radial mean value.

defined by

$$
\begin{aligned}
& u_{m=0, z, s}=0.5 \cdot\left(u_{m=0, z}(z, r)-u_{m=0, z}(-z, r)\right) \\
& u_{m=0, r, s}=0.5 \cdot\left(u_{m=0, r}(z, r)+u_{m=0, r}(-z, r)\right) .
\end{aligned}
$$

Of course, equivalent is the antisymmetric part defined by

$$
\begin{aligned}
& u_{m=0, z, a}=0.5 \cdot\left(u_{m=0, z}(z, r)+u_{m=0, z}(-z, r)\right) \\
& u_{m=0, r, a}=0.5 \cdot\left(u_{m=0, r}(z, r)-u_{m=0, r}(-z, r)\right) .
\end{aligned}
$$

Equivalent to $E_{m=0, i} \boldsymbol{u}_{0, s}$ can be used to cover only the symmetric energy in $m=$ 0, $E_{m=0, s, i}$, which is also done in fig. 3.37 (dashed line) for $E_{m=0, s, z} / E_{m \neq 0, z}$. For precession rates from $\Omega_{P}=-0.17$ to $-0.14 E_{m=0, z} / E_{m \neq 0, z}$ and the symmetric part $E_{m=0, s, z} / E_{m \neq 0, z}$ are nearly identical. The energy is essentially bound in the double roll. From $\Omega_{P}=-0.13$ to -0.09 the proportional amount of energy in symmetric components varies, with a minimum around $\Omega_{P}=-0.11$. The remaining energy must be bound in antisymmetric components. This is due to a breakdown of the $m=0$-modes as it can be seen in fig. 3.38: The variation of the energy of the meridional components $E_{m=0, z}+$ $E_{m=0, r}$ and the azimuthal component $E_{m=0, \phi}$ are shown over time. At $\Omega_{P}=-0.16$ (fig. 3.38a) the values keep relatively stable with small variations, at $\Omega_{P}=-0.11$ (fig. 3.38b) both parameters, $E_{m=0, z}+E_{m=0, r}$ and $E_{m=0, \phi}$, fluctuate chaotically over time. Here, time spans exist with the total energy in the meridional components differing strongly 


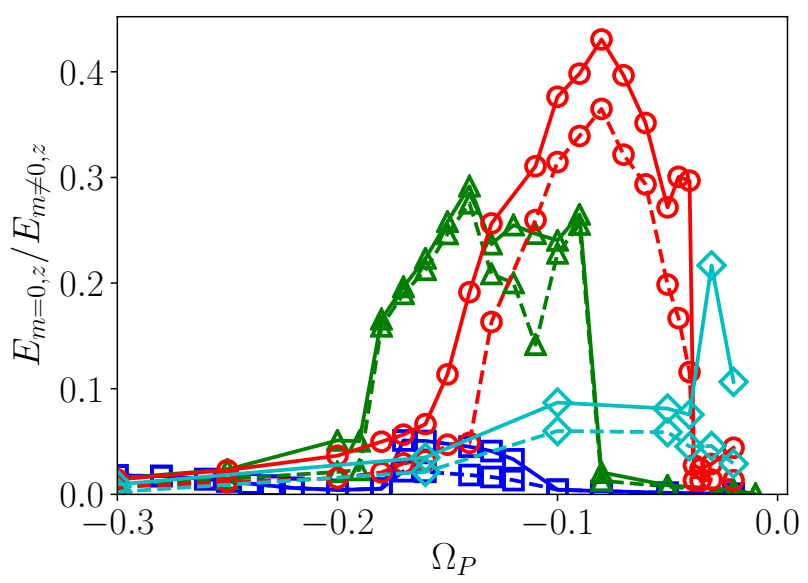

Figure 3.37: Non-zero amounts of $E_{m=0, z} / E_{m \neq 0, z}$ (continuous line) exist for $\mathrm{Ek}=2.5$. $10^{-4}, \mathrm{Ek}=1.0 \cdot 10^{-4}$ only in the same region of $\Omega_{P}$ like the middle region presented in sec. 3.1. For $\mathrm{Ek}=1.0 \cdot 10^{-5}$ the amount is much smaller than by higher Ekman numbers, except for the peak by $\Omega_{P}=-0.03$, which also occurs at $\mathrm{Ek}=1.0 \cdot 10^{-4}, \Omega_{P}=-0.04$. Considering only the $\mathrm{x}-\mathrm{y}-$ plane-symmetric energy $E_{m=0, z, s} / E_{m \neq 0, z}$ (dashed line) which leads to the double roll described in 3.35 , the peak is not seen. For $\mathrm{Ek}=1.0 \cdot 10^{-3}$ $E_{m=0, z} / E_{m \neq 0, z}$ seems to correlate with the middle region, too, but shows other behavior in antisymmetric amounts and also in non-zero amounts below the lower limit.

from the symmetric share. In fig. 3.39 streamline representations of the meridional velocity

$$
\boldsymbol{u}_{p o l}=u_{m=0, z} \hat{z}+u_{m=0, r} \hat{\boldsymbol{r}}
$$

and the symmetric and antisymmetric amounts $\boldsymbol{u}_{p o l, s}=u_{m=0, z, s} \hat{\boldsymbol{z}}+u_{m=0, r, s} \hat{\boldsymbol{r}}$ and $\boldsymbol{u}_{p o l, a}=u_{m=0, z, a} \hat{\boldsymbol{z}}+u_{m=0, r, a} \hat{\boldsymbol{r}}$ for two different points in time $t=89.9$ (left side) and $t=250.1$ (right side) regarding the time scale in fig. $3.38 \mathrm{~b}$ are shown. The symmetric components consist in both cases of the double roll as it was already seen in fig. 3.35, however, additionally a strong roll extending over the whole z-axis emerges in the antisymmetric components, counteracting part of the double roll in the total components. While the direction of flow of the double roll is the same over the whole time - an inwards flow at the equatorial plane and an outwards flow at the top and bottom the antisymmetric flow changes direction at the chosen points of time. Over a long time 
(a) $\mathrm{Ek}=2.5 \cdot 10^{-4}, \Omega_{P}=-0.16$

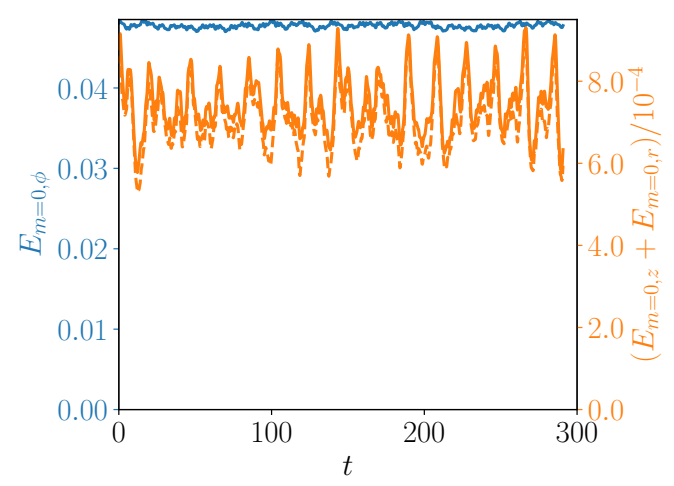

(c) $\mathrm{Ek}=2.5 \cdot 10^{-4}, \Omega_{P}=-0.09$

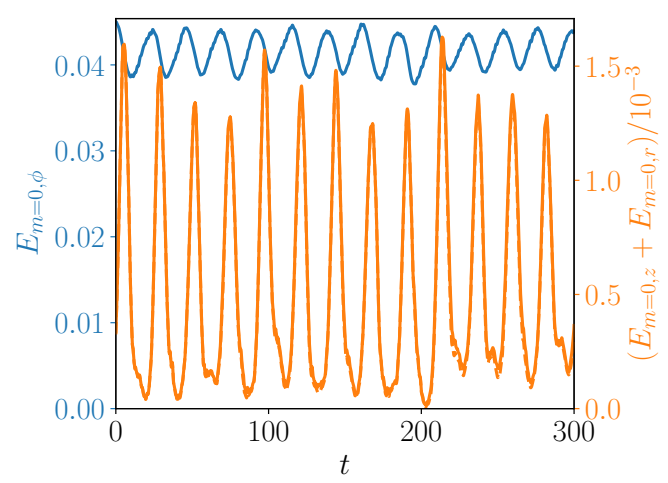

(b) $\mathrm{Ek}=2.5 \cdot 10^{-4}, \Omega_{P}=-0.11$

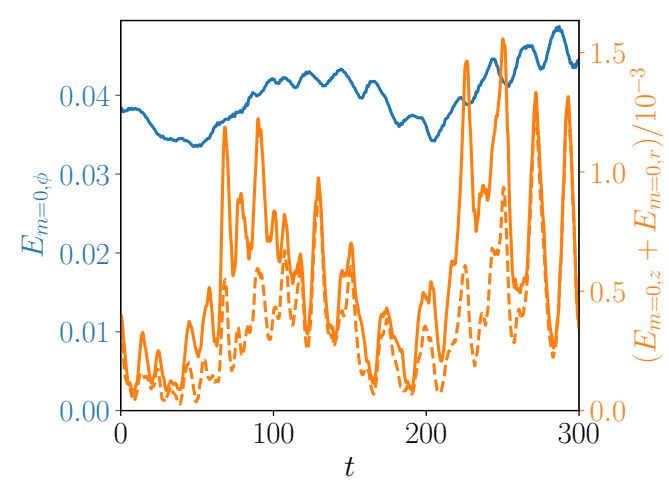

Figure 3.38: Time evolution of $E_{m=0, \phi}$ and $\left(E_{m=0, z}+E_{m=0, r}\right)$ at $\mathrm{Ek}=2.5 \cdot 10^{-4}$ for $\Omega_{P}=-0.16,-0.11,-0.09$ with a dashed line indicating the symmetric parts of $\left(E_{m=0, z}+E_{m=0, r}\right)$ : every precession rate shows a different behavior in energy of axisymmetric modes, stable, a breakdown to chaotic behavior with recognizable antisymmetric amount and oscillating. 

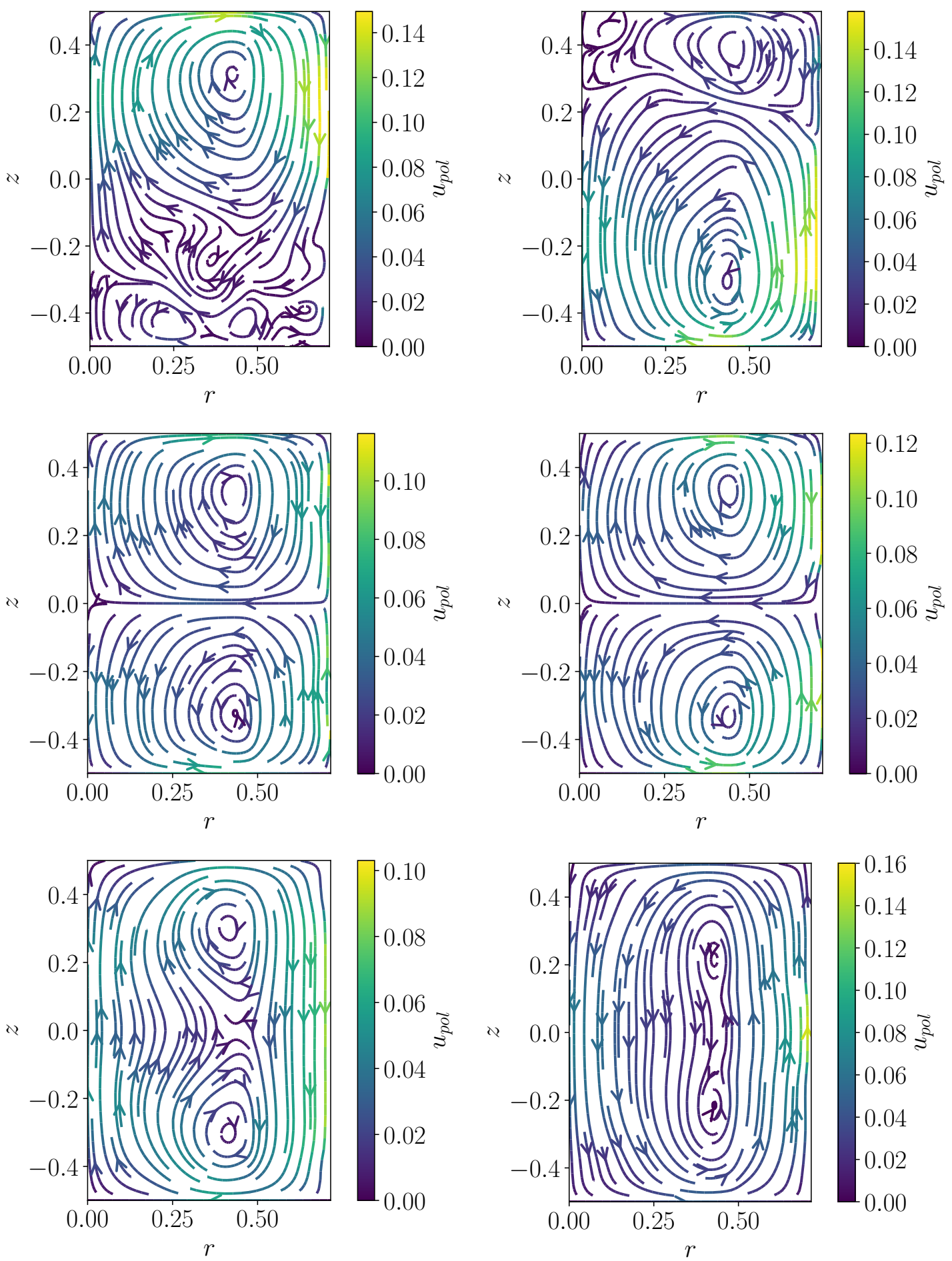

Figure 3.39: $\boldsymbol{u}_{p o l}, \boldsymbol{u}_{p o l, s}$ and $\boldsymbol{u}_{p o l, a}$ (from top to bottom) at Ek $=2.5 \cdot 10^{-4}, \Omega_{P}=-0.11$ at time points $t=89.9$ (left side) and $t=250.1$ (right side). 
of observation, both directions of flow surface for equal amount and would cancel each other out in a time-mean flow. The breakdown, which leads to the chaotic structure in fig. $3.38 \mathrm{~b}$ for $\mathrm{Ek}=2.5 \cdot 10^{-4}$ and $\Omega_{P}=-0.11$, is also characterized by a strong oscillating state of energy as it can be seen here for $t>200$. Looking at $\Omega_{P}=-0.09$ in fig. $3.38 \mathrm{c}$, this state is observable without any breakdown occurring. Furthermore, no antisymmetric energy emerge, making this only a feature of the breakdown. This strong oscillatory behavior can be observed for a wide array of parameters, at Ek $=2.5 \cdot 10^{-4}$ for $\Omega_{P}=-0.09,-0.1,-0.12,-0.13$. The angular frequency of this oscillation, $\omega_{m=0}$, increases when the precession ratio becomes stronger and is shown for some parameter values in fig. 3.40. Independent of the Ekman number, $\omega_{m=0}$ increases with increasing

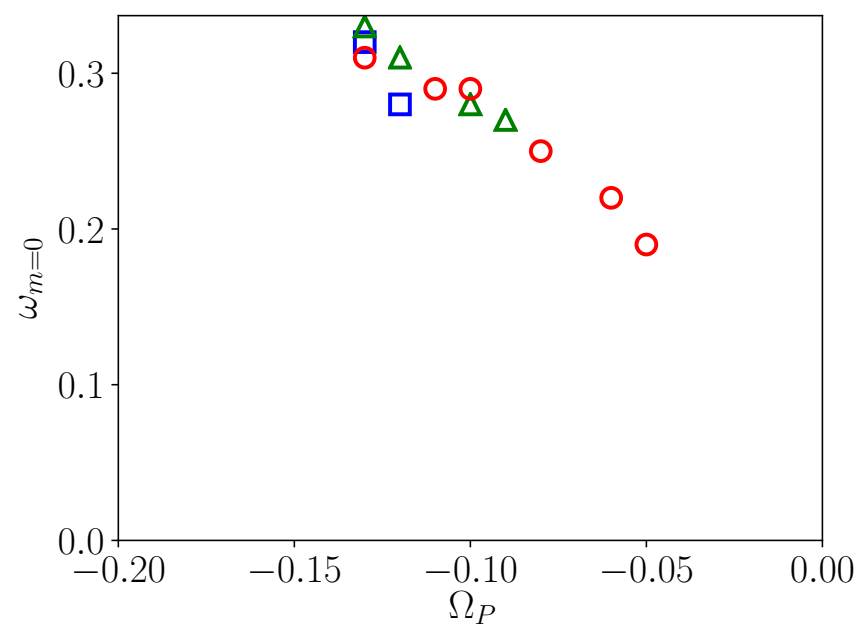

Figure 3.40: Angular frequency of axisymmetric oscillations $\omega_{m=0}$ at $\mathrm{Ek}=3.0 \cdot 10^{-4}$ (squares), $\mathrm{Ek}=2.5 \cdot 10^{-4}$ (triangles) and $\mathrm{Ek}=1.0 \cdot 10^{-4}$ (circles) for different $\Omega_{P}$ is constant for variation of Ekman number but increases with increasing precession rate.

precession rate, but getting more chaotic with decreasing Ekman number.

At $\mathrm{Ek}=1.0 \cdot 10^{-4}$ and $\Omega_{P}=-0.08$ in fig. 3.41a the oscillating behavior is recognizable and provides the highest ratio of $E_{m=0, z} / E_{m \neq 0, z}$ in fig. 3.37, while at $\Omega_{P}=-0.04$ the amount of antisymmetric energy is relatively high compared to that of symmetric energy and peaks locally for $E_{m=0, z} / E_{m \neq 0, z}$. In fig. $3.41 \mathrm{~b}$ the breakdown is more extensive and for even lower Ekman number like in fig. 3.41d - for $\mathrm{Ek}=1.0 \cdot 10^{-5}$ and $\Omega_{P}=-0.03-$ it is predominant. Consequently the antisymmetric components in $E_{m=0, z} / E_{m \neq 0, z}$ are higher, too. Nevertheless it is furthermore the same flow structure than at Ek $=2.5 \cdot 10^{-4}$ and $\Omega_{P}=-0.11$ as can be seen in in fig. 3.42a. For $E_{m=0, z} / E_{m \neq 0, z}$ at this Ekman 
(a) $\mathrm{Ek}=1.0 \cdot 10^{-4}, \Omega_{P}=-0.08$

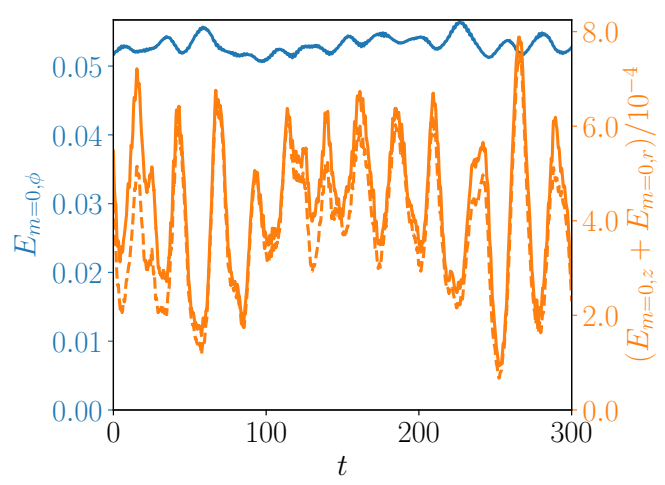

(c) $\mathrm{Ek}=1.0 \cdot 10^{-5}, \Omega_{P}=-0.05$

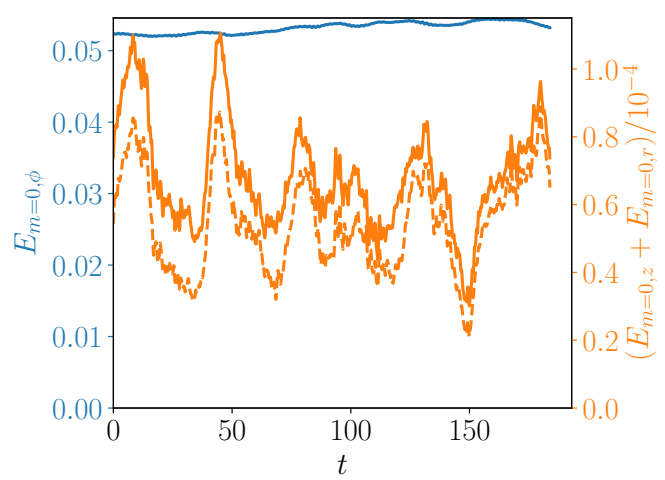

(b) $\mathrm{Ek}=1.0 \cdot 10^{-4}, \Omega_{P}=-0.04$

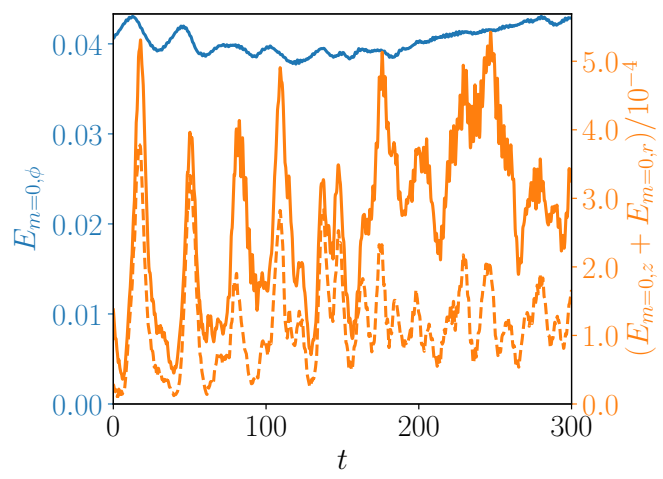

(d) $\mathrm{Ek}=1.0 \cdot 10^{-5}, \Omega_{P}=-0.03$

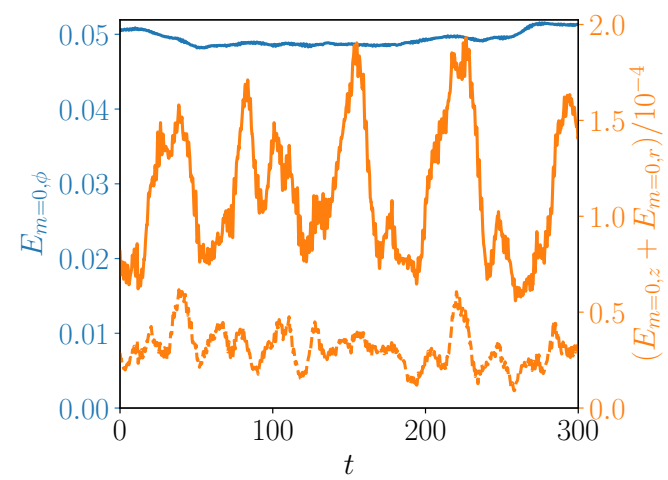

Figure 3.41: Time evolution of $E_{m=0, \phi}$ and $\left(E_{m=0, z}+E_{m=0, r}\right)$ at $\mathrm{Ek}=1.0 \cdot 10^{-4}$ (top) $\Omega_{P}=-0.08$ (left side) and $\Omega_{P}=-0.04$ (right side) and $\mathrm{Ek}=1.0 \cdot 10^{-4}$ (bottom) $\Omega_{P}=-0.05$ (left side) and $\Omega_{P}=-0.03$ (right side) with a dashed line indicating the symmetric parts of $\left(E_{m=0, z}+E_{m=0, r}\right)$ : The behavior of axisymmetric energy becomes more chaotic at lower Ekman numbers and breakdowns appear more often. 
(a) $\mathrm{Ek}=1.0 \cdot 10^{-5}, \Omega_{P}=-0.05$

(b) $\mathrm{Ek}=1.0 \cdot 10^{-5}, \Omega_{P}=-0.03$
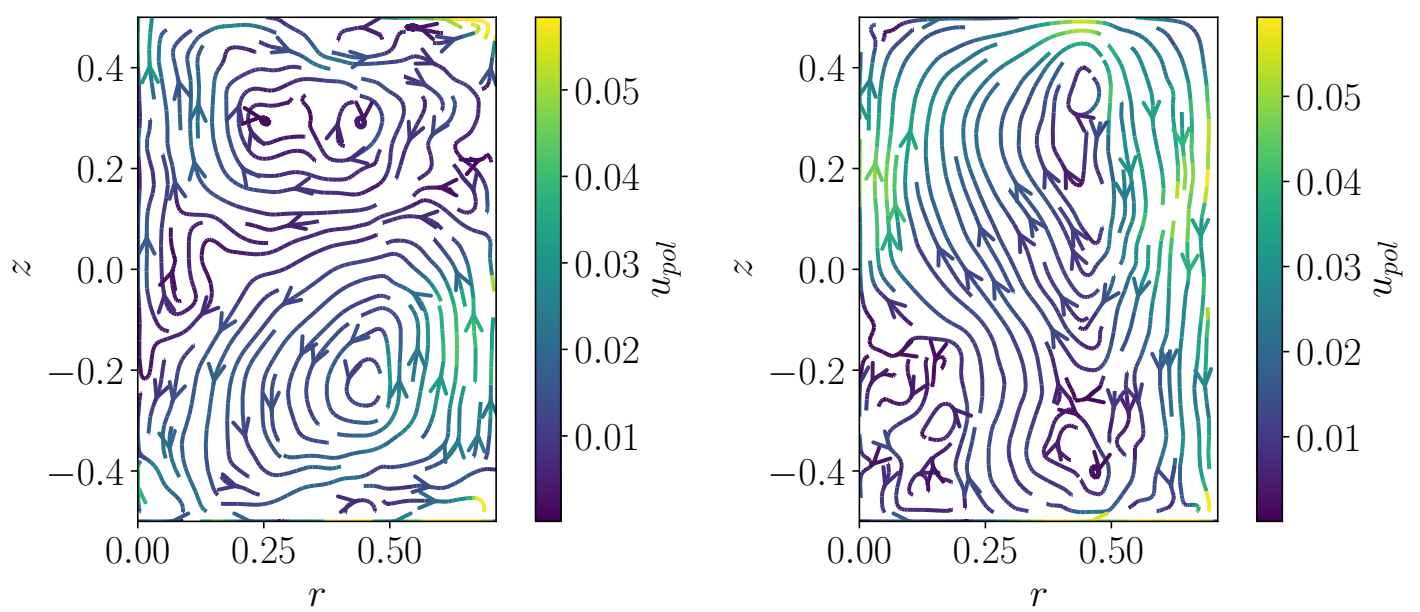

Figure 3.42: $\boldsymbol{u}_{p o l}$ at $\mathrm{Ek}=1.0 \cdot 10^{-5}$ for $\Omega_{P}=-0.05$ (left side) and $\Omega_{P}=-0.03$ (right side): At $\Omega_{P}=-0.05$ the axisymmetric flow mainly consists of the double roll, while at $\Omega_{P}=-0.3$ the antisymmetric single roll predominant is in the flow.

number this constitutes the highest reachable value, while the predominantly symmetric modes at $\Omega_{P} \leq-0.04$ (see fig. $3.42 \mathrm{~b}$ for $\Omega_{P}=-0.05$ ) are small compared to the values achieved for $\mathrm{Ek} \geq 1.0 \cdot 10^{-4}$ and mostly chaotic in the course over time in 3.41c. The $E_{m=0, z} / E_{m \neq 0, z}$ at $\mathrm{Ek}=1.0 \cdot 10^{-3}$ shows a very different behavior to that of lower Ekman numbers: After a well defined area between $\Omega_{P}=-0.1$ and -0.17 , with mixed symmetric and antisymmetric $m=0$ energy ratio, the total ratio drops significantly and only a symmetric share persists (see fig. 3.43).

Comparing the regions of the single vortex state of sec. 3.3, especially in fig. 3.32 with the ones of axisymmetric modes, one notices the overlapping range between both. This is demonstrated in fig. 3.44 with marked areas in orange for regions with $R_{\text {vortex }}>0.01$ and blue areas for regions with $E_{m=0, z} / E_{m \neq 0, z}>0.1$. While at high Ekman numbers the amount of axisymmetric energy is high for a broad range of precession rates, it is almost congruent for $E k \leq 1.0 \cdot 10^{-4}$. There is no parameter combination for the whole examined range, where a vortex as defined in sec. 3.3 exists, but no axisymmetric energy below a certain threshold. Further, in fig. 3.45 the axisymmetric meridional energy $E_{m=0, r}+E_{m=0, z}$ is presented for a chosen set of parameters. At $\mathrm{Ek}=3.0 \cdot 10^{-4}$ and $\Omega_{P}=-0.13$ (see fig. 3.45a) one can see that the frequency of the occurrence of the vortex is the same as the oscillation of the axisymmetric energy. For slightly lower 


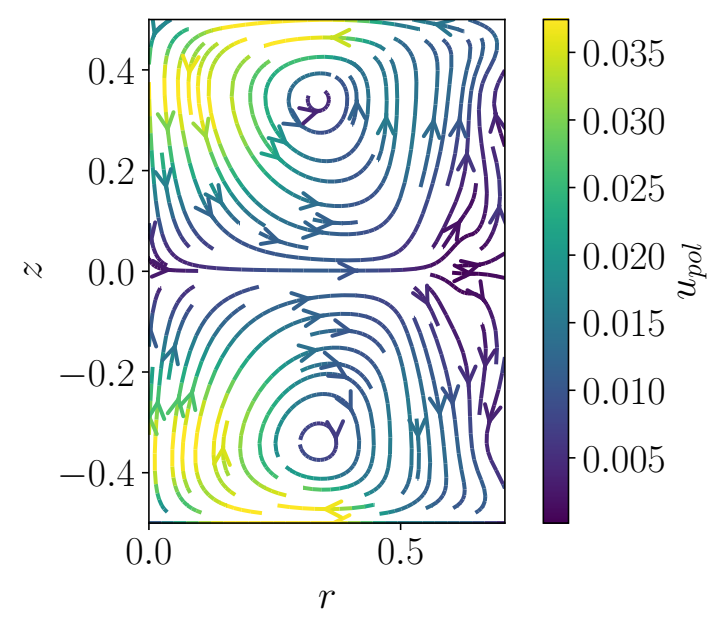

Figure 3.43: At lower precession rates $\Omega_{P} \leq-0.18$ the antisymmetric component vanishes for $\mathrm{Ek}=1.0 \cdot 10^{-3}$ and only the double roll as seen here for $\Omega_{P}=-0.3$ persist.

Ekman numbers as at $\mathrm{Ek}=2.5 \cdot 10^{-4}$ and $\Omega_{P}=-0.13$ this holds true (see fig. $3.45 \mathrm{~b}$ ), and when $R_{\text {vortex }}$ is greater than zero, it possesses the same wavelength than $E_{m=0, r}+E_{m=0, z}$ for the same parameters. In the range $\Omega_{P}=-0.18$ to -0.14 at $\mathrm{Ek}=2.5 \cdot 10^{-4}$ with only constant $E_{m=0, r}+E_{m=0, z}$, no occurrence of the single vortex state is noted. For lower Ekman numbers both quantities become chaotic over time as already discussed here and in sec. 3.3 and no such periodic behavior can be observed anymore or any other relation between those two (see fig. $3.45 \mathrm{c}$ for $\mathrm{Ek}=1.0 \cdot 10^{-4}$ and $\Omega_{P}=-0.13$ and fig. $3.45 \mathrm{~d}$ for $\mathrm{Ek}=1.0 \cdot 10^{-5}$ and $\left.\Omega_{P}=-0.02\right)$. This breakdown regime occurs for lowering Ekman number but also for increasing precession rates, marking a transition into chaotic turbulence, as can be seen in fig. 3.46. For Ek $\leq 2.0 \cdot 10^{-4}$ no stable modes occur anymore, instead the oscillating phase and the breakdown broadens over the range of precession rates by decreasing Ek. For Ek $\leq 5.0 \cdot 10^{-5}$ axisymmetric modes only appear in the chaotic breakdown phase. For $\mathrm{Ek} \geq 1.0 \cdot 10^{-4}$ the single vortex state as defined in sec. 3.3 only exists simultaneously to the oscillating phase, for $\mathrm{Ek} \leq 5.0 \cdot 10^{-5}$ only to the breakdown regime.

After all, the dominant axisymmetric structures can be described by the two structures $s_{1} t_{1}$ and $s_{2} t_{1}$ sketched in fig. 3.47 , which separates roughly into a azimuthal $\left(u_{0, \phi}\right)$ and meridional $\left(u_{0, r}+u_{0, z}\right)$ motion. The azimuthal motion is essentially the geostrophic circulation as seen in fig. 3.34 and does increase with decreasing Ek or increasing precession rate (fig. 3.48). This corresponds with the kinetic energy $E_{k i n}$ in fig. 3.4 and 


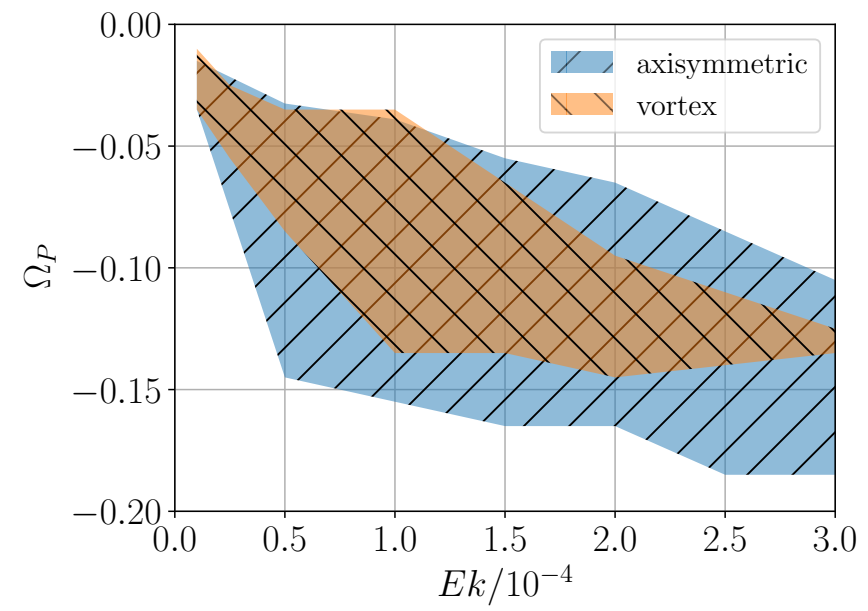

Figure 3.44: Map of regions with axisymmetric modes and single vortex state: Blue area defines occurrence of axisymmetric modes with $E_{m=0, z} / E_{m \neq 0, z}>0.1$ and orange areas show the single vortex state with $R_{\text {vortex }}>0.01$.

at low Ek the geostrophic motion makes up to 0.8 and more of the total kinetic energy, showing the sudden increase in kinetic energy at the critical $\Omega_{P}$ in fig. 3.5 is mostly due to this circulation.

But the relation between this and the meridional components is another measure of interest, since it is known from other results [Dudley, 1989, Ravelet et al., 2005], that a good ratio of poloidal to toroidal motion - which roughly translate to the ratio of meridional to azimuthal motion in the structures described here - can have a great influence on dynamo action and the one used here is a good equivalent to such a ratio. To implement this, the energy of the different components, $E_{m=0, i}$, defined in eq. 3.9 , is accordingly set in a meridional-to-azimuthal ratio as

$$
\Gamma_{0}=\left(E_{m=0, r}+E_{m=0, z}\right) / E_{m=0, \phi}
$$

For the different Ekman numbers examined, $\Gamma_{0}$ is maximal at Ekman $=2.5 \cdot 10^{-4}$ with $\Gamma_{0} \approx 0.015$ in the region of $\Omega_{P}=-0.18$ to -0.14 , while for lower Ekman numbers its value decreases overall until at $\mathrm{Ek}=1.0 \cdot 10^{-5}$ it is lower than 0.002 for all $\Omega_{P}$ as it can be seen in figure 3.49. If $\Gamma_{0}$ is of any importance to the dynamo effect, its effect becomes diminished to lower Ekman numbers.

For the purpose of comparison with the experiments of [Giesecke et al., 2018] mentioned at the beginning of this section, some simulation runs were done with no-slip boundary 
(a) $\mathrm{Ek}=3.0 \cdot 10^{-4}, \Omega_{P}=-0.13$

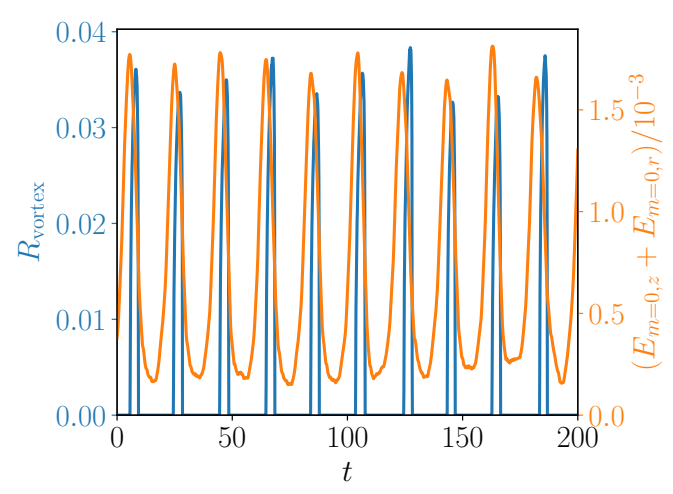

(c) $\mathrm{Ek}=1.0 \cdot 10^{-4}, \Omega_{P}=-0.13$

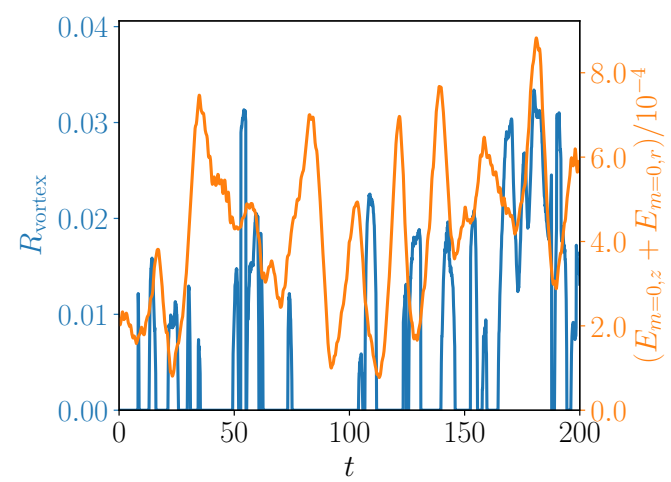

(b) $\mathrm{Ek}=2.5 \cdot 10^{-4}, \Omega_{P}=-0.13$

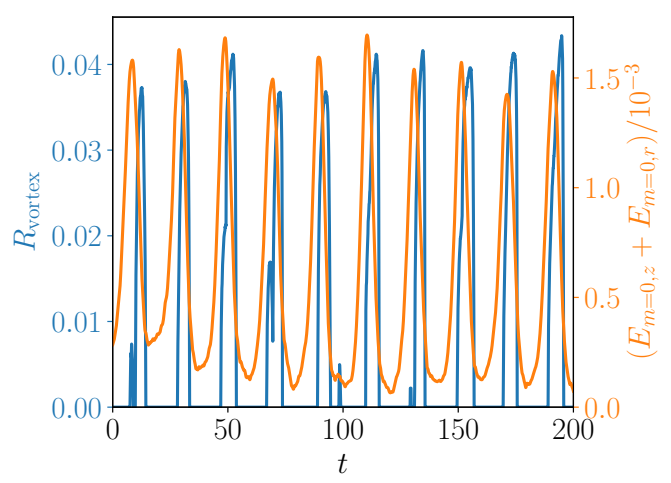

(d) $\mathrm{Ek}=1.0 \cdot 10^{-5}, \Omega_{P}=-0.02$

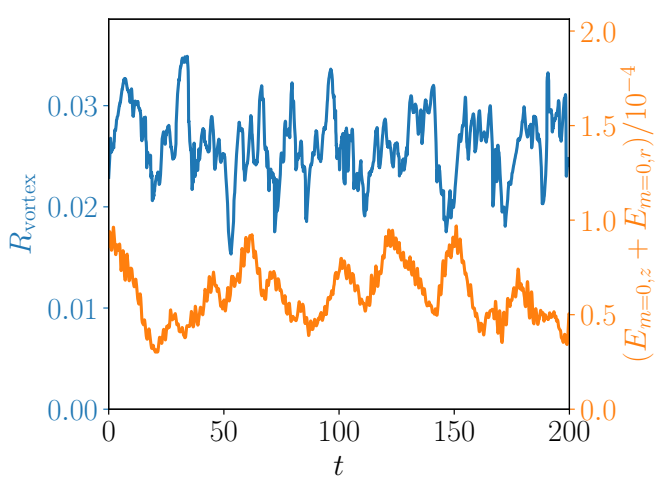

Figure 3.45: Comparison of time evolution of $E_{m=0, r}+E_{m=0, z}$ and $R_{\mathrm{vortex}}$ for different parameters: Oscillations in $E_{m=0, r}+E_{m=0, z}$ occur parallel to oscillations in $R_{\text {vortex }}$ with the same frequency, at breakdowns, both become chaotic, but with no notable correlation. 


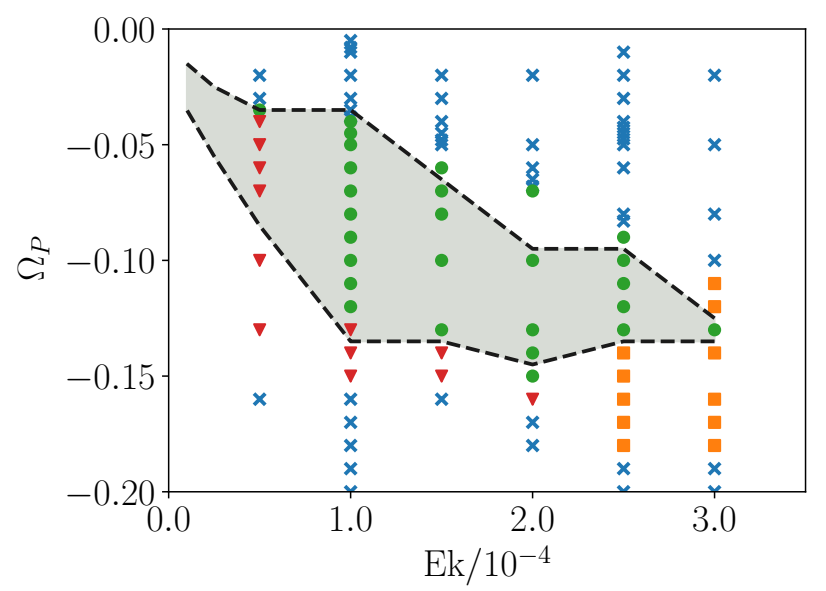

Figure 3.46: The different phases of axisymmetric modes for different $\Omega_{P}$ from -0.2 to -0.01 and $\mathrm{Ek}$ from $5.0 \cdot 10^{-5}$ to $3.0 \cdot 10^{-4}$ : Time independent stable (squares), oscillating (circles), chaotic breakdown of (triangles) and nonexistent modes (crosses). The single vortex regime of sec. 3.3 is highlighted in grey.

conditions. The geometry of the boundaries, however, is kept as a cube. The boundary condition was modified to set all velocity components boundary points to zero as described in sec. 2.2.1. The chosen parameters are $\mathrm{Ek}=2.5 \cdot 10^{-4}, \Omega_{P}=-0.16$ and $\Omega_{P}=-0.12$ as well as $\mathrm{Ek}=1.0 \cdot 10^{-4}, \Omega_{P}=-0.1$, instances of stable, oscillating and breakdown behavior under free-slip boundary conditions. For all three no-slip cases, $\Gamma_{0}$ is above the value it holds for the free-slip cases. This is mainly due to the azimuthal motion being counteracted by the strict boundaries and thus reducing $E_{m=0, \phi} . E_{m=0, z}$ shows much less variation as can be seen in tab. 3.1. Looking at the evolution over time, the axisymmetric motion is more stable in the case of no-slip boundary conditions: $u_{m=0, z}$ does not vary as much as in the case of free-slip boundary conditions. In fig. 3.50 the actual curves of $u_{m=0, z}$ for points in time over 50 rotations at $r=0.45$ is displayed. The curves for $\mathrm{Ek}=2.5 \cdot 10^{-4}, \Omega_{P}=-0.12$ spread more around the mean value for the free-slip case (fig. 3.50a) than the no-slip case (fig. 3.50b), which shows very little variation at all, being stable instead of oscillating. At lower Ek the variation increases for the no-slip case, too, as can be seen in fig. 3.50d for no-slip boundaries at $\mathrm{Ek}=1.0 \cdot 10^{-4}, \Omega_{P}=-0.1$. However, it is still stable, while with free-slip boundaries the breakdown generate much more variation. Simulations run for lower Ekman numbers are too expensive at calculation time to further examine this change of behavior with 

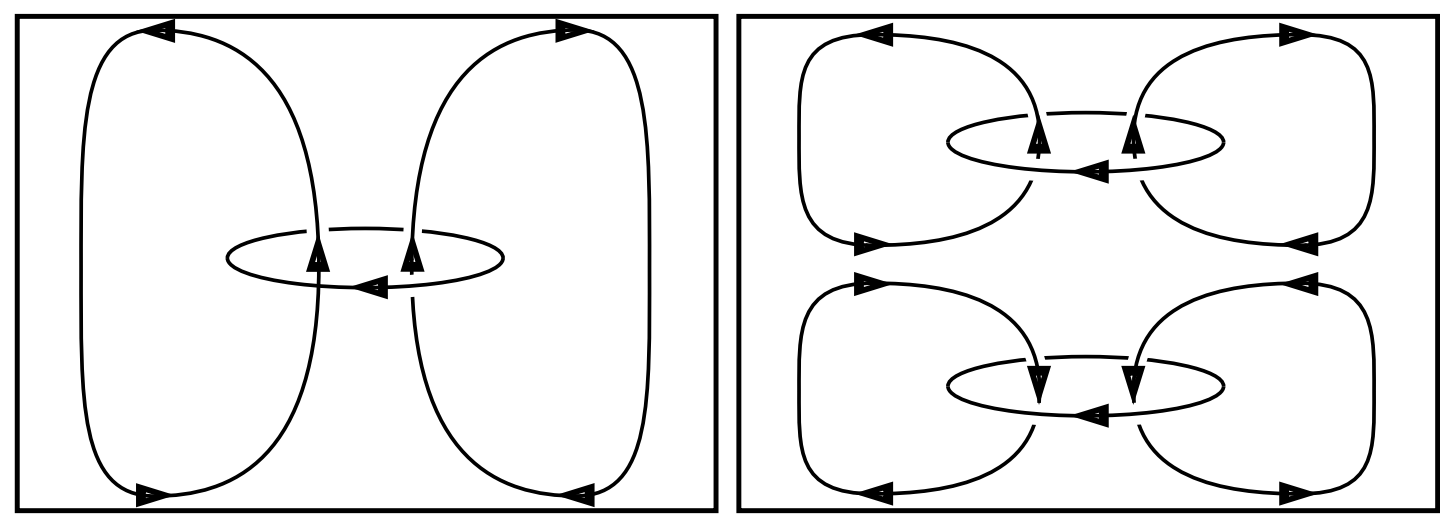

Figure 3.47: The main two modes in $m=0$ occurring are sketched here: $m=0 k=1$ (here known as $\left.s_{1} t_{1}\right)$ on the right side, $m=0 k=2\left(s_{2} t_{1}\right)$ on the left side.

\begin{tabular}{cc|cc|cc|cc} 
& & \multicolumn{2}{|c|}{$E_{m=0, z}$} & \multicolumn{2}{|c|}{$\Gamma_{0}$} & \multicolumn{2}{c}{$E_{m=0, z} / E_{m \neq 0, z}$} \\
\hline Ek & $\Omega_{P}$ & free-slip & no-slip & free-slip & no-slip & free-slip & no-slip \\
$2.5 \cdot 10^{-4}$ & -0.12 & $3.83 \cdot 10^{-4}$ & $2.65 \cdot 10^{-4}$ & 0.015 & 0.049 & 0.28 & 0.05 \\
$2.5 \cdot 10^{-4}$ & -0.16 & $3.88 \cdot 10^{-4}$ & $5.00 \cdot 10^{-4}$ & 0.016 & 0.070 & 0.23 & 0.08 \\
$1.0 \cdot 10^{-4}$ & -0.10 & $2.70 \cdot 10^{-4}$ & $2.57 \cdot 10^{-4}$ & 0.009 & 0.046 & 0.37 & 0.04
\end{tabular}

Table 3.1: Mean values of $E_{m=0, z}, \Gamma_{0}$ and $E_{m=0, z} / E_{m \neq 0, z}$ over 50 rotations for three different parameter combinations with no-slip boundary conditions and the equivalent data with free-slip boundary conditions.

no-slip boundaries.

\subsection{Triad resonance}

At this point, little is understood about the form of laminar flow inside the cube, but the equation of motion given by eq. 2.18 is mostly homogeneous, except the inhomogeneous Poincaré force $F_{P}$ (see eq. 2.14), which is centrosymmetric to the origin. Additionally, the boundaries of the cube - and the boundary conditions - are invariant under reflections to the origin. Hence, any stable laminar flow inside the cube should follow the symmetry condition

$$
\boldsymbol{u}(\boldsymbol{r})=-\boldsymbol{u}(-\boldsymbol{r}) .
$$




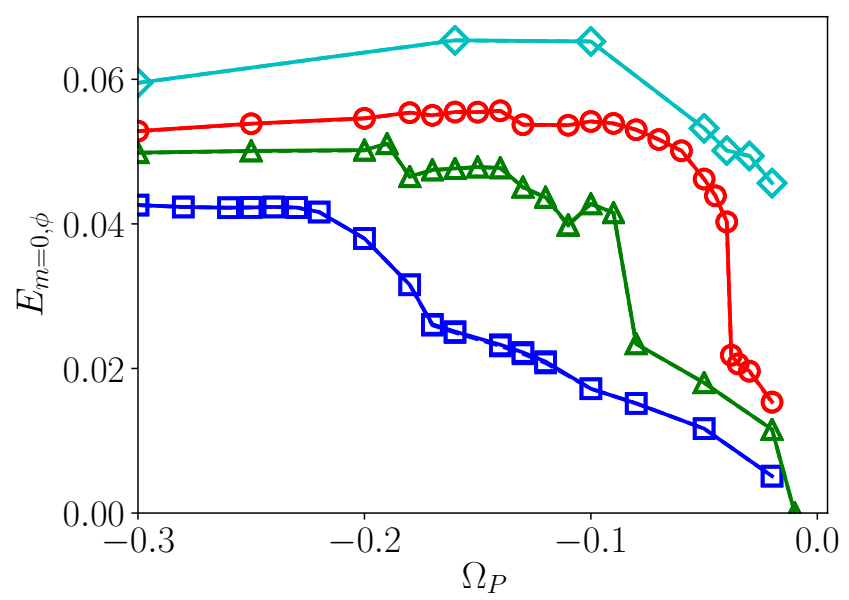

Figure 3.48: $E_{m=0, \phi} \quad$ for $\quad \mathrm{Ek}=1.0 \cdot 10^{-3} \quad$ (squares), $\quad \mathrm{Ek}=2.5 \cdot 10^{-4} \quad$ (triangles), $\mathrm{Ek}=1.0 \cdot 10^{-4}$ (circles), $\mathrm{Ek}=1.0 \cdot 10^{-5}$ (diamonds) is the main contribution to total kinetic energy $E_{k i n}$ (see fig. 3.4).

Any kind of discrepancy from this symmetry is a clear indicator for an instability in the flow. Therefore it is favorable to split any observed flow into symmetric and antisymmetric parts similar as it was done by Lorenzani et al. [Lorenzani and Tilgner, 2001, Lorenzani and Tilgner, 2003] in spheroidal cavities, so that

$$
\boldsymbol{u}(\boldsymbol{r})=\boldsymbol{u}_{s}(\boldsymbol{r})+\boldsymbol{u}_{a}(\boldsymbol{r})
$$

with $\boldsymbol{u}_{s}(\boldsymbol{r})=\frac{\boldsymbol{u}(\boldsymbol{r})-\boldsymbol{u}(-\boldsymbol{r})}{2}$ and $\boldsymbol{u}_{a}(\boldsymbol{r})=\frac{\boldsymbol{u}(\boldsymbol{r})+\boldsymbol{u}(-\boldsymbol{r})}{2}$. Of course, not every instability has to generate an antisymmetric component $\boldsymbol{u}_{a}(\boldsymbol{r})$ different from zero as it can be observed by the double roll in sec. 3.4. To simplify this to one parameter an energy based on the antisymmetric components can be calculated:

$$
E_{a}=\frac{1}{V}\left\langle\int_{V} \frac{1}{2} u_{a}^{2} \mathrm{~d} V^{\prime}\right\rangle_{t} .
$$

Fully symmetric instabilities are possible, but at least any non-trivial $E_{a}$ must be due to an instability.

The actual values for simulations can be seen in fig. 3.51, where $E_{a}$ for different Ekman numbers ranging from $10^{-3}$ to $10^{-5}$ are plotted against the precession rates $\Omega_{P}$. Here, all Ekman numbers below $10^{-3}$ are unstable for great $\left|\Omega_{P}\right|$, while at an Ekman number of $10^{-3}$ only a small, sharply defined section exists, where the flow becomes unstable by the means of antisymmetric components. This section coincides with the resurgence 


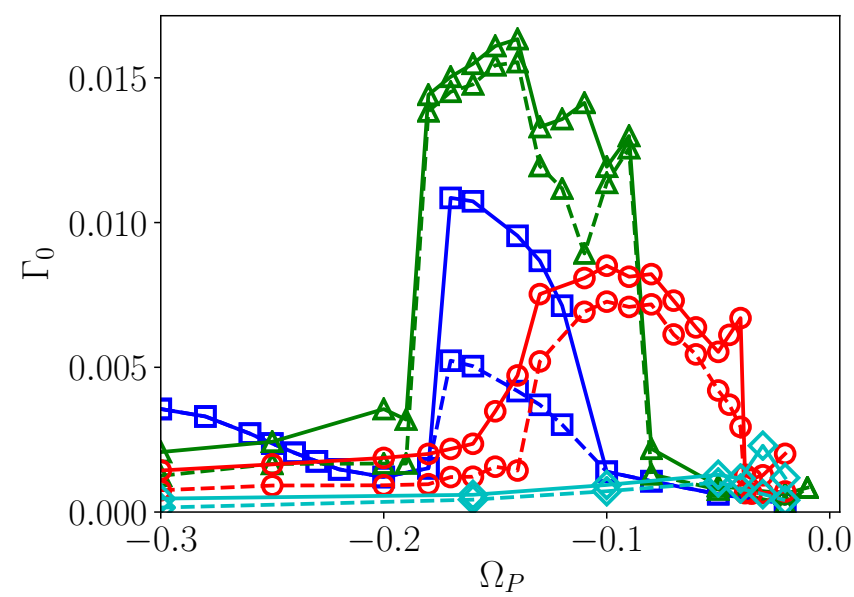

Figure 3.49: The relation $\Gamma_{0}=\left(E_{m=0, r}+E_{m=0, z}\right) / E_{m=0, \phi}$ is qualitatively similar to fig. 3.37, but decreases overall with decreasing Ekman number after achieving its highest value at $\mathrm{Ek}=2.5 \cdot 10-4$. The dashed line shows only the symmetric part $E_{m=0, s, r}+E_{m=0, s, z} / E_{m=0, \phi}$, while the continuous line shows the total value of $E_{m=0, r}+E_{m=0, z} / E_{m=0, \phi}$. Differences between the two indicate existence of a $s_{1} t_{1}$-mode.

of the $s_{1} t_{1}$-flow at Ek $=10^{-3}$, which is also antisymmetric with respect to symmetry through the origin and is seemingly connected to this effect. At lower Ekman numbers other effects arise and at $\mathrm{Ek}=2.5 \cdot 10^{-4}$ the antisymmetric energy at different precession rates parts into two different regimes: $\Omega_{P} \leq-0.045$ and $-0.01>\Omega_{P}>-0.03$.

Again, a good reference frame here is the fluid reference system in 3.17. The antisymmetric velocity $\boldsymbol{u}_{a}(\boldsymbol{r})$ can be treated in this reference frame to inspect the components alongside $\boldsymbol{z}^{\prime}$ and $\boldsymbol{r}^{\prime}$, adjusting the system to cylindrical coordinates $\left(r^{\prime}, \varphi^{\prime}, z^{\prime}\right)$. Looking at planes perpendicular to $\omega_{F}$ in the regime around $\Omega_{P}=-0.02$ like in fig. 3.53 , one notices the symmetry around the center of four at $u_{a, z^{\prime}}$ and the symmetry of five at $u_{a, r^{\prime}}$. The symmetry in $u_{a, r^{\prime}}$ can be extended to the antisymmetric vorticity defined as

$$
\boldsymbol{\omega}_{a}(\boldsymbol{r})=\frac{1}{2}((\nabla \times \boldsymbol{u}(\boldsymbol{r}))+(\nabla \times \boldsymbol{u}(-\boldsymbol{r})))=\frac{1}{2}\left(\nabla \times \boldsymbol{u}_{a}(\boldsymbol{r})\right)
$$

as seen in 3.54, which holds the same symmetry. This symmetry can very likely be compared to the mode structure sketched in 3.52.

This bears similarity to instabilities driven by triad resonance, where two free inertial waves are driven by the spin-over mode forced by the precession. This was discussed by 
(a) $\mathrm{Ek}=2.5 \cdot 10^{-4}, \Omega_{P}=-0.12$, free-slip

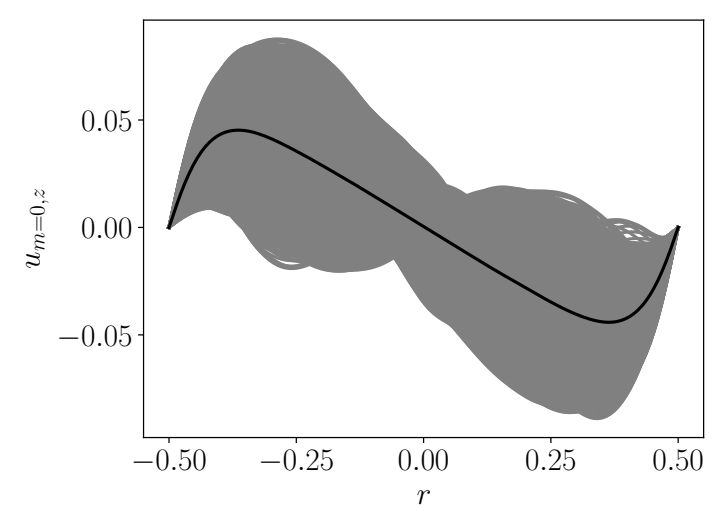

(c) $\mathrm{Ek}=1.0 \cdot 10^{-4}, \Omega_{P}=-0.1$, free-slip

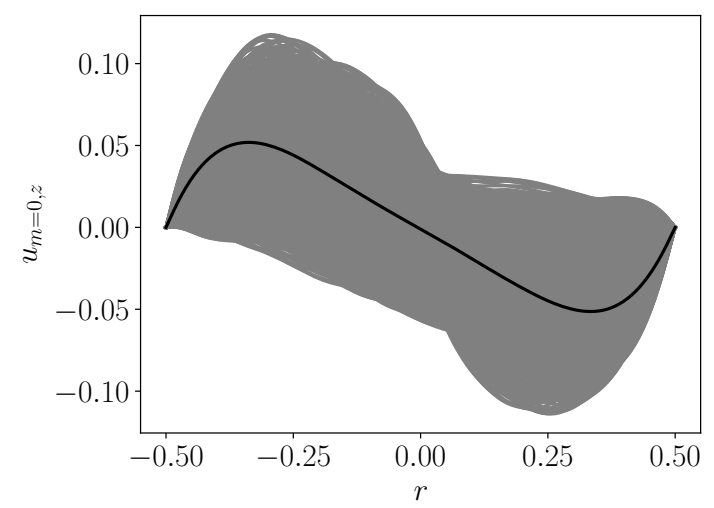

(b) $\mathrm{Ek}=2.5 \cdot 10^{-4}, \Omega_{P}=-0.12$, no-slip

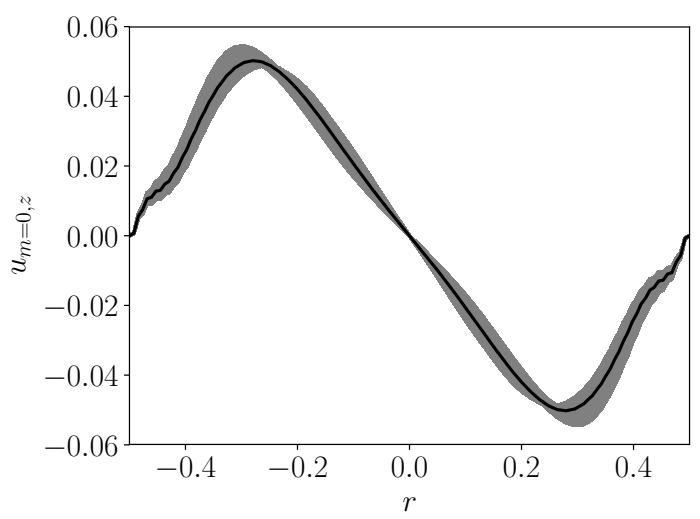

(d) $\mathrm{Ek}=1.0 \cdot 10^{-4}, \Omega_{P}=-0.1$, no-slip

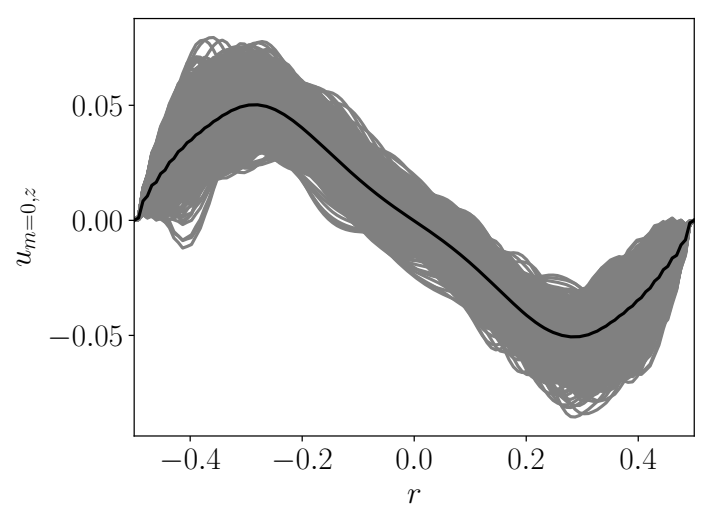

Figure 3.50: Curves of $u_{m=0, z}(r)$ for roughly 3000 points in time over 50 rotations (grey lines) and their mean value (black line) for free-slip (left side) and no-slip (right side) boundary conditions at $\mathrm{r}=0.45$ : For both shown parameter combinations, $\mathrm{Ek}=2.5 \cdot 10^{-4}, \Omega_{P}=-0.12($ top $)$ and $\mathrm{Ek}=1.0 \cdot 10^{-4}, \Omega_{P}=$ -0.1 (bottom), the no-slip cases show less variation from the mean value, which shows the form matching the double roll. 


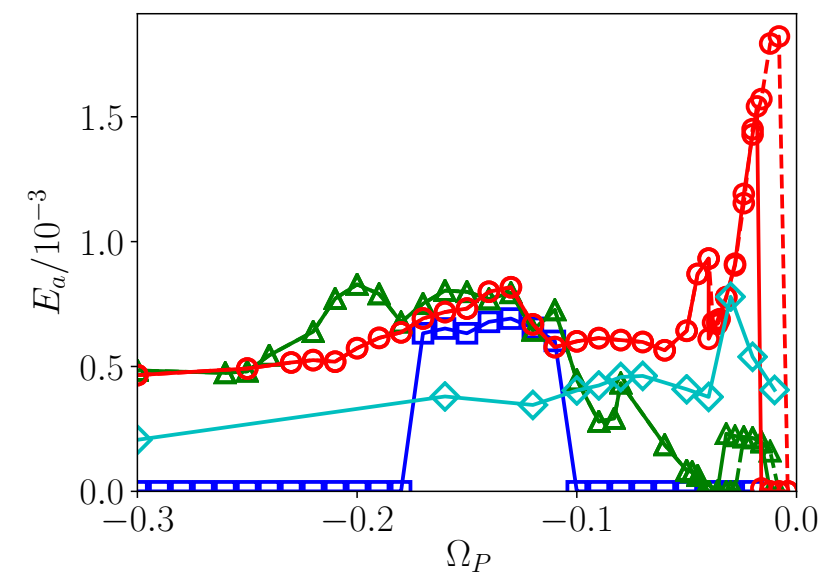

Figure 3.51: $E_{a}\left(\Omega_{P}\right)$ behaves different for the varying Ekman numbers: Ek $=1.0 \cdot 10^{-3}$ (squares) only has a sharp defined area with $E_{a} \neq 0$, while at $\mathrm{Ek}=2.5 \cdot 10^{-4}$ (triangles) two different regions can be identified. $\mathrm{Ek}=1.0 \cdot 10^{-4}$ (circles) and $\mathrm{Ek}=1.0 \cdot 10^{-5}$ (diamonds) have antisymmetric components over the whole examined region except at very small precession rates. Also they peak each for $\left|\Omega_{P}\right| \leq 0.03$. The different line styles indicates hysteresis effects: The dashed lines originate from changing $\Omega_{P}$ to zero from left to right in the figure while continuous line is obtained from the opposite direction.

Kerswell [Kerswell, 1999] and seen in experiments [Lagrange et al., 2008, Lagrange, Romain et al., 2009]. For this to happen, the free modes must adhere to some specific conditions: The difference of their wave numbers and eigenfrequencies must yield the wave numbers and frequencies of the third mode, so in the case of the spin-over-mode with axial and azimuthal wave number equal to one

$$
\begin{aligned}
m_{1}-m_{2} & =1, \\
\omega_{1}-\omega_{2} & =\omega_{D}
\end{aligned}
$$

with $m_{1}, m_{2}$ the azimuthal wave numbers of the free modes and $\omega_{1}, \omega_{2}$ for the frequencies. The difference of one between the modes observed is a strong indication for a triad resonance. Looking at a cylindrical surface around $\hat{\boldsymbol{z}}^{\prime}$ at a radius $R=0.3$ in fig. 3.55, $u_{a, z^{\prime}}$ and $u_{a, r^{\prime}}$ do have the same azimuthal wave numbers, the axial structure is much more complex due to the constraints by the boundaries.

The second regime at $\Omega_{P} \leq-0.045$ shows the azimuthal wave numbers 2 for $u_{a, z^{\prime}}$, while $\omega_{a, z^{\prime}}$ shows a wave number of 1 (fig. 3.56), the simplest mode possible for a triad 


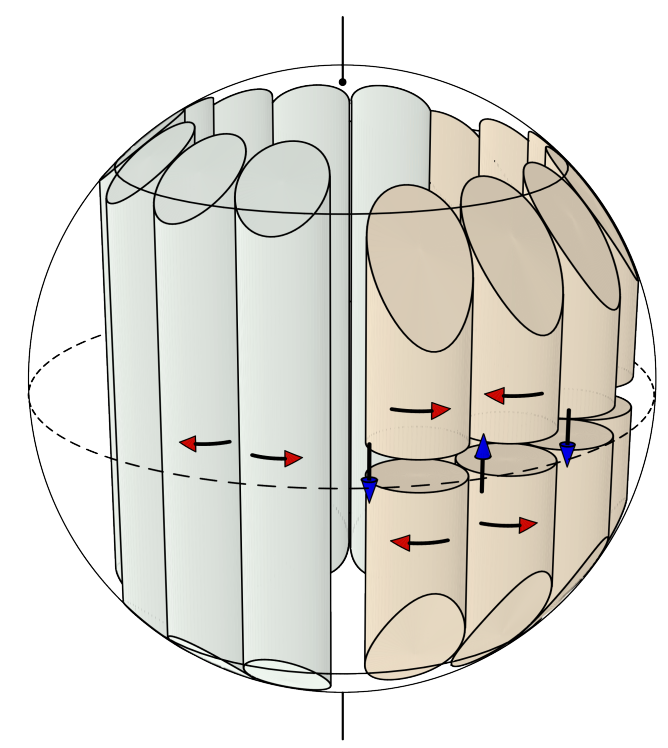

Figure 3.52: sketch of unstable modes coupled in a triad resonance by viscous instabilities from [Lorenzani, 2002].

resonance. This instability continues to exist even after $\Omega_{P} \leq-0.09$, when the double roll appears as seen in fig. 3.57. For $\Omega_{P} \leq-0.19$ the fluid devolves completely into a turbulent state, where no azimuthal modes are apparent anymore.

The antisymmetric energy $E_{a}$ for $(4,5)$-mode, once saturated, oscillates (see fig 3.58) with four times the precession frequency, which shows a adherence to the fourfold symmetry of the boundaries. For the $(1,2)$-mode at $\Omega_{P} \leq-0.045 E_{a}$ stays small at the onset, but quickly gains a chaotic time dependence at lower $\Omega_{P}$. Low enough in fig. 3.59, the variance of the energy increases more, but at $\Omega_{P} \in[-0.18,-0.14]$, it decreases without lowering the mean antisymmetric energy (see fig. 3.51) and enabling the strong $m=0$-mode visible in fig. 3.49 .

At $\mathrm{Ek}=1.0 \cdot 10^{-4}$ the antisymmetric energy does not separate into different regions, but is roughly on the same level for all precession rates with the exception of very low $\left|\Omega_{P}\right|$, where a strong peak appears. Interestingly, the maximum of the peak is dependent on a hysteresis the system shows with variation of $\Omega_{P}$ : For increasing $\left|\Omega_{P}\right|$ the instability starts first at $\Omega_{P}=-0.018$, while it does not end before $\Omega_{P}=-0.008$ when decreasing precession rates to zero. To generate this hysteresis loop in fig. 3.51, as starting point for the curve in positive direction $\left(\Omega_{P} \rightarrow 0\right) \Omega_{P}=-0.04$ was chosen, for negative direction $\left(\Omega_{P} \rightarrow \infty\right) \Omega_{P}=0$. The instability can be attributed to a triad resonance with a 

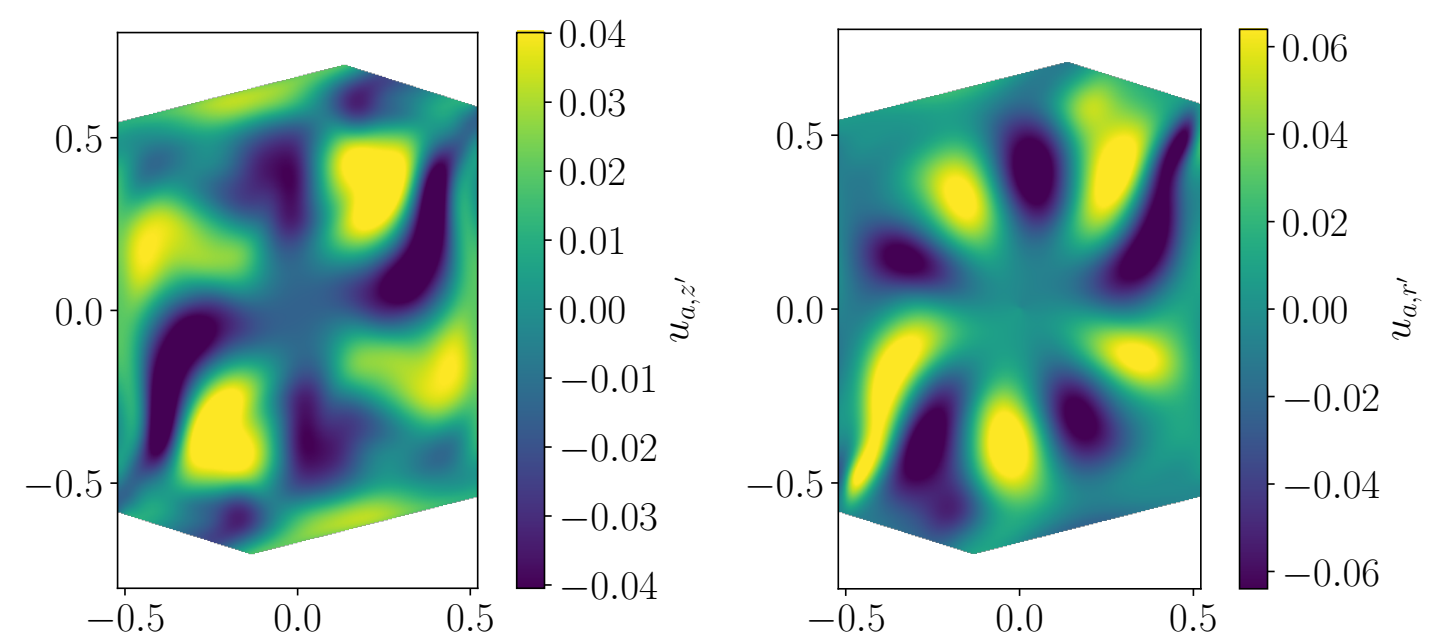

Figure 3.53: $u_{a, z^{\prime}}$ (left) and $u_{a, r^{\prime}}$ (right) for $\mathrm{Ek}=2.5 \cdot 10^{-4}$ and $\Omega_{P}=-0.02$ in the plane normal to $\boldsymbol{z}^{\prime}$ through the origin. A azimuthal 4-mode in $u_{a, z^{\prime}}$ and a 5 -mode in $u_{a, r^{\prime}}$ is visible.

pair of modes with $(1,2)$ in azimuthal direction as can be seen in 3.60 . In $\omega_{a, z^{\prime}}$ the mode in azimuthal direction is 1 and 2 for $u_{a, z^{\prime}}$. Both modes extend in axial direction through the whole container (see fig. 3.61), not showing any sign of a higher structure alongside this axis. At $\Omega_{P}=-0.008, E_{a}(t)$ is stable and oscillating over time, at $\Omega_{P}=-0.02$, however, it already gained a chaotic component (see fig. 3.62) like it happens at $\mathrm{Ek}=2.5 \cdot 10^{-4}$ for higher $\left|\Omega_{P}\right|$. Similarly, the modes in $u_{a, z^{\prime}}$ and $\omega_{a, z^{\prime}}$ become more chaotic fast in fig. 3.63 and 3.64 with the latter at $\Omega_{P}=-0.04$, when $s_{2} t_{1}$ is also present in the flow.

The second condition $\omega_{1}-\omega_{2}=\omega_{D}$ is examined by looking at the temporal change of the velocity $u_{\text {mantle }}$ at a fixed point inside the cube. The point $\boldsymbol{r}_{t}=(x, y, z)=(0.25,0.5,0.5)$ was chosen and the velocity was split into $\boldsymbol{u}_{a}(t)$ and $\boldsymbol{u}_{s}(t)$ as defined in 3.19. With data from roughly 1600 rotations, a power density spectrum was calculated based upon components along the $z$-axis and the radial component. The spectrum is based on the Welch method with eight overlapping periodograms and normalized to the peak of the spin-over mode which occurs in $u_{s}$ components.

The results for $\mathrm{Ek}=1.0 \cdot 10^{-4}, \Omega_{P}=-0.008$ are presented in figure 3.65 for both the stable branch in the hysteresis in fig. 3.51 with $\left|\Omega_{P}\right|$ increasing and the unstable branch with $\left|\Omega_{P}\right|$ decreasing. The stable branch shows only one peak around $\omega=1.004$, which is equivalent to the forced spin-over mode and rotates with $\omega_{D}$. No other notable features 


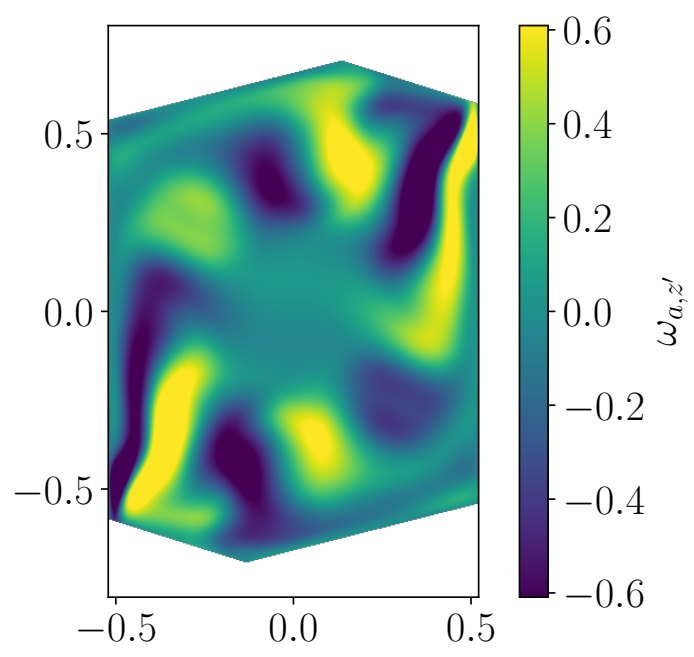

Figure 3.54: $\omega_{a, z^{\prime}}$ for $\mathrm{Ek}=2.5 \cdot 10^{-4}$ and $\Omega_{P}=-0.02$ in the plane normal to $\boldsymbol{z}^{\prime}$ through the origin.

can be seen, so other effects can be ruled out. On the unstable branch, on the other hand, more significant frequencies occur: At 0.180 for the spectra of $u_{a, r}$ and 1.183 for $u_{a, z}$ can be assigned to the azimuthal modes 1 and 2 respectively seen in figure 3.60. This is confirmed by observing the motion of the modes for 100 rotations in a plane $z=0$ through the origin. Also the difference between both give roughly $\omega_{D}$, the result of the second condition for triad resonance. Additionally two other pairs can be seen: At 0.45 in the spectrum of $u_{a, z}$ and 0.54 of $u_{a, r}$, while at 0.35 and 1.72 two frequencies in the symmetric components appear. Since these are not seen in the spectrum of the stable branch, it is very likely connected to the triad resonance. Interestingly, the two additional antisymmetric frequencies add up to the rotation rate, so it could be a second, weak triad, where one of the modes is actual retrograde, so that, as an example, $\omega_{1}-\omega_{2}=0.46-$ $(-0.54) \approx \omega_{D}$. But the small proportion in comparison to the remaining modes makes it impossible to distinguish it in pictures of $u_{a, z^{\prime}}$ and $\omega_{a, z^{\prime}}$ in figure 3.60 with the naked eye. The frequencies in the spectra of symmetric components $u_{s, z}$ with $\omega_{1}=1.71$ and $u_{s, r}$ with $\omega_{2}=0.36$ on the other hand would be a good candidate for a triad resonance originating from an elliptical instability. Here the difference between frequencies and modes has to be two[Kerswell, 1993, Lorenzani and Tilgner, 2003]. Possibly, these are the effect of more complex interaction of the instability with the boundaries. For higher precession rates like $\Omega_{P}=-0.035$ the power density spectrum is simplified, as only two different frequencies in the antisymmetric components remain (see figure 3.66 (left side)). Again, these can be attributed to the azimuthal modes 1 and 2 in $u_{a, z^{\prime}}$ and $\omega_{a, z^{\prime}}$, but 

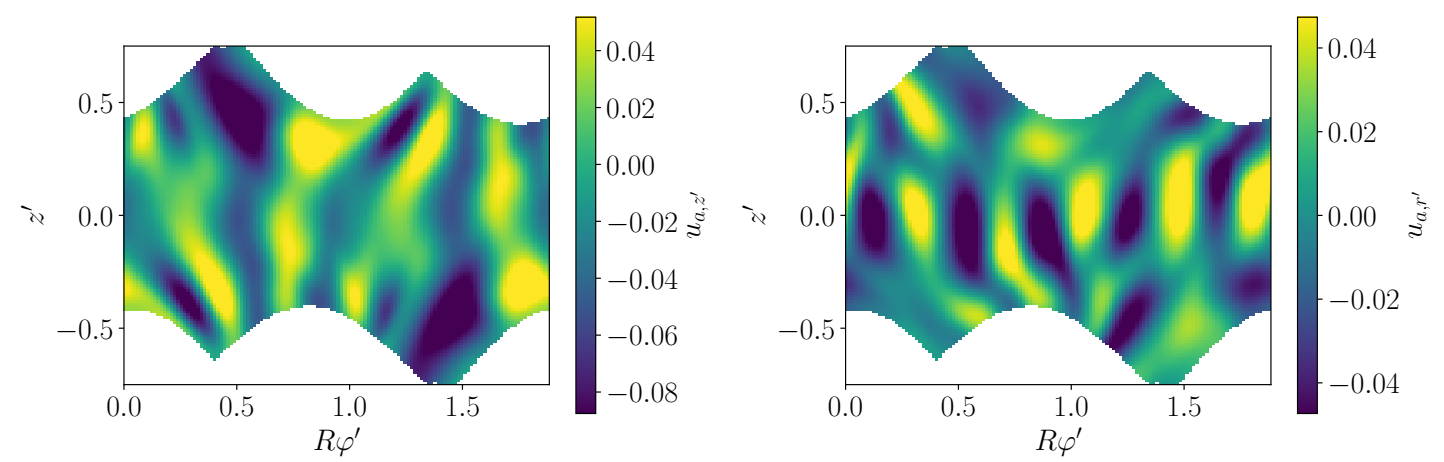

Figure 3.55: $u_{a, z^{\prime}}$ (left) and $u_{a, r^{\prime}}$ (right) for $\mathrm{Ek}=2.5 \cdot 10^{-4}$ and $\Omega_{P}=-0.02$ on a cylindrical surface along $\boldsymbol{z}^{\prime}$ and radius $R=0.3$. The same modes as in fig. 3.53 are visible in azimuthal direction, but in $z^{\prime}$-direction the antisymmetric flow grows much more complex because of the constraints by the boundary.

they increased both in comparison to $\Omega_{P}=-0.008$ for some $\Omega_{P}$. These values are noted in tab. 3.2 and still fulfill the condition in eq. 3.23. Naturally, the shift in frequencies is caused by the increasing perturbation the precession induces into the system.

\begin{tabular}{ccccc}
$\Omega_{P}$ & -0.008 & -0.02 & -0.03 & -0.035 \\
\hline$u_{a, r}$ & 0.180 & 0.278 & 0.348 & 0.374 \\
$u_{a, z}$ & 1.183 & 1.28 & 1.365 & 1.389
\end{tabular}

Table 3.2: frequencies $\omega$ for triads at $\mathrm{Ek}=1.0 \cdot 10^{-4}$

For $\mathrm{Ek}=2.5 \cdot 10^{-4}$ they show also signs of triad resonances in their spectra: In figure 3.66 (right side) for $\Omega_{P}=-0.02$ two frequencies at $\omega_{1}=2.19$ and $\omega_{2}=1.10$ in the spectra of $u_{a, z}$ and $u_{a, r}$ fit the second condition and are likely attributes of the azimuthal modes of 4 and 5 at figure 3.53. A third frequency at $\omega \approx 0.02$ may be a relic of the precession frequency. For $\Omega_{P}=-0.05$ with the azimuthal modes $k_{1}=1$ and $k_{2}=2$ like in 3.56 one can find quickly peaks in figure 3.67 similar to the ones at Ek $=1.0 \cdot 10^{-4}$ with $\omega_{1}=0.33$ and $\omega_{2}=1.39$ fulfilling the second condition. The additional peak at 1.8 implies additional effects in play, but while it is of similar magnitude as the other triad peaks, it can not be identified in the fluid properly. At higher precession rates the flow assumes more chaotic features as seen before and the power density spectrum looses evidence for one dominant triad, while further showing indications for triads in azimuthal modes as seen in figure 3.56. 

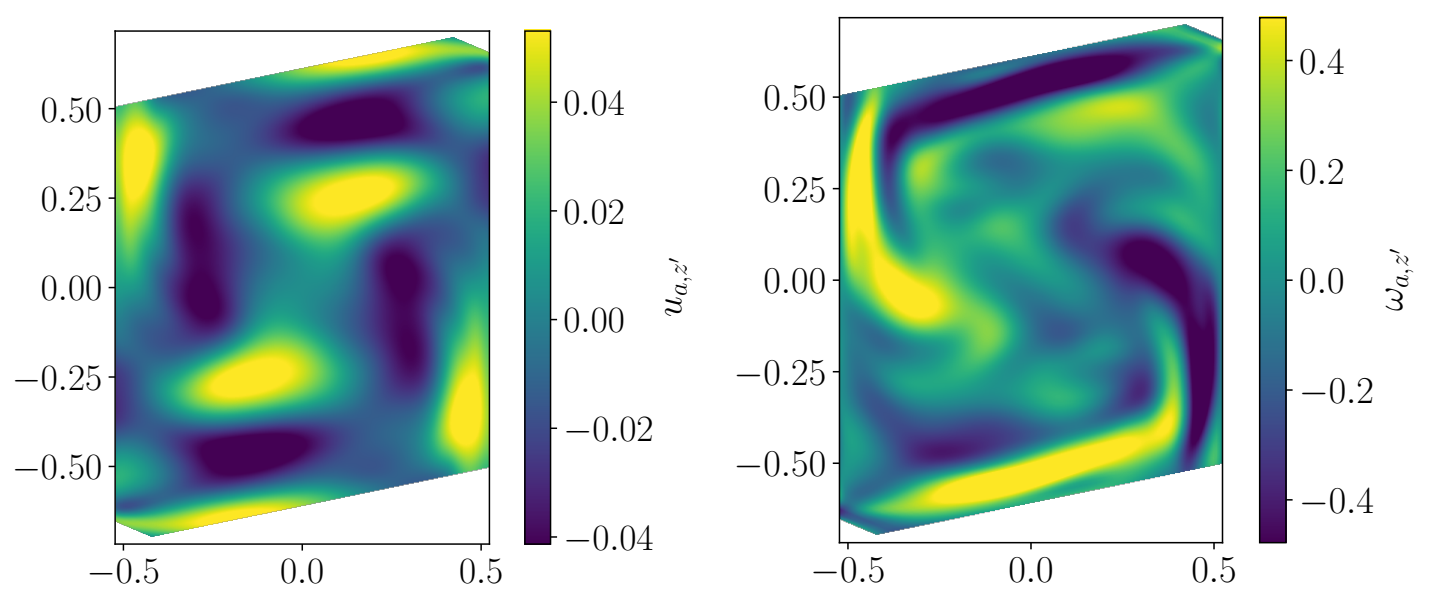

Figure 3.56: $u_{a, z^{\prime}}$ (left) and $\omega_{a, z^{\prime}}$ (right) for $\mathrm{Ek}=2.5 \cdot 10^{-4}$ and $\Omega_{P}=-0.08$ in the plane normal to $\boldsymbol{z}^{\prime}$ through the origin. A azimuthal 2-mode in $u_{a, z^{\prime}}$ and a 1-mode in $\omega_{a, z^{\prime}}$ is visible.

At $\mathrm{Ek}=1.0 \cdot 10^{-5}$ the antisymmetric energy $E_{a}$ is always non-zero for all simulated values of $\Omega_{P}$, hence, no stable flow is possible at this Ekman number. Of course, at small distance to $\Omega_{P}=0$ marginalized flows on top of the solid-body rotation are possible but of no interest and no simulations were done beyond $\Omega_{P}=-0.01$. In opposition to higher Ekman numbers, here $E_{a}$ is never bound to any triad resonances in the flow. The instabilities needed for it must originate from other mechanisms and tends to become turbulent very fast. The additional antisymmetric energy notable between $\Omega_{P}=-0.03$ and -0.01 stems from the not centrosymmetric motion of the central vortex at this parameters seen in fig. 3.26 and the $s_{1} t_{1}$-mode there. 

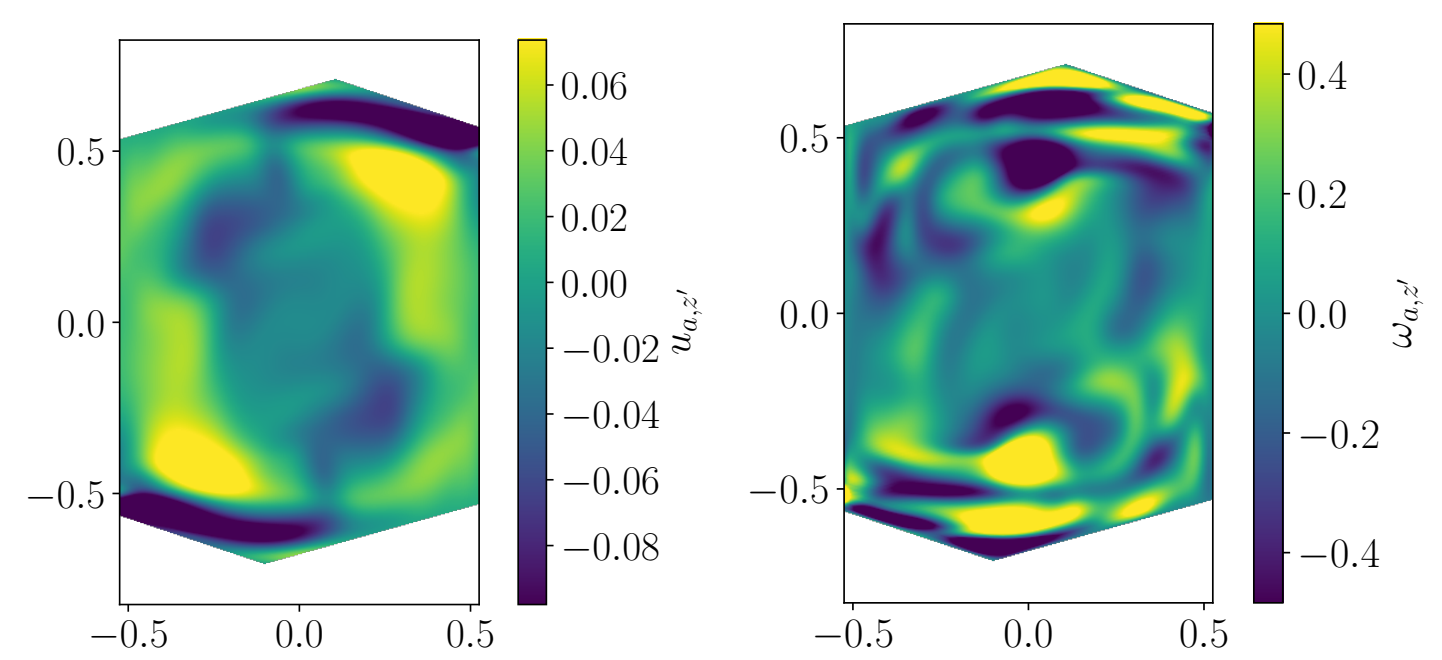

Figure 3.57: $u_{a, z^{\prime}}$ (left) and $\omega_{a, z^{\prime}}$ (right) for $\mathrm{Ek}=2.5 \cdot 10^{-4}$ and $\Omega_{P}=-0.16$ in the plane normal to $\boldsymbol{z}^{\prime}$ through the origin. A azimuthal 2-mode in $u_{a, z^{\prime}}$ and a 1-mode in $\omega_{a, z^{\prime}}$ is visible.

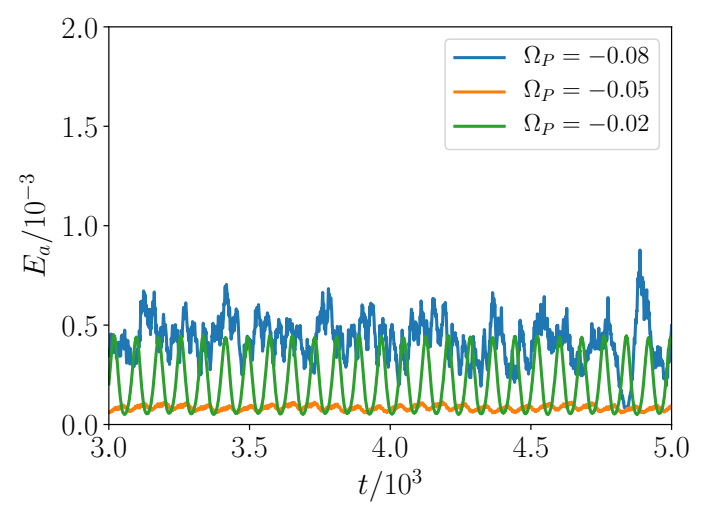

Figure 3.58: $E_{a}(t)$ at $\mathrm{Ek}=2.5 \cdot 10^{-4}$ for the first unstable flow around $\Omega_{P}=-0.02$ oscillates, while at the second occurrence of antisymmetric energy at lower precession rates $E_{a}(t)$ is small at the unset like at $\Omega_{P}=-0.05$ and quickly gains a chaotic time dependence when increasing $\left|\Omega_{P}\right|$. 


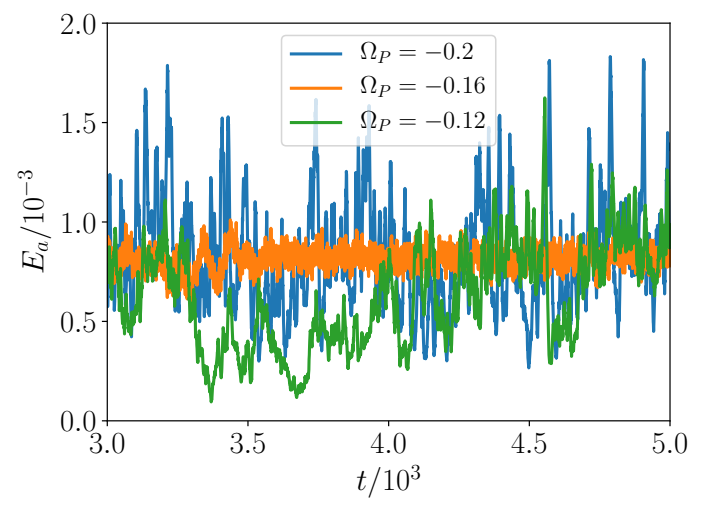

Figure 3.59: $E_{a}(t)$ at $\mathrm{Ek}=2.5 \cdot 10^{-4}$ at $\Omega_{P}<-0.08$ remains chaotic in nature, with the special case around $\Omega_{P} \approx-0.16$ which, while chaotic, shows much less variance.
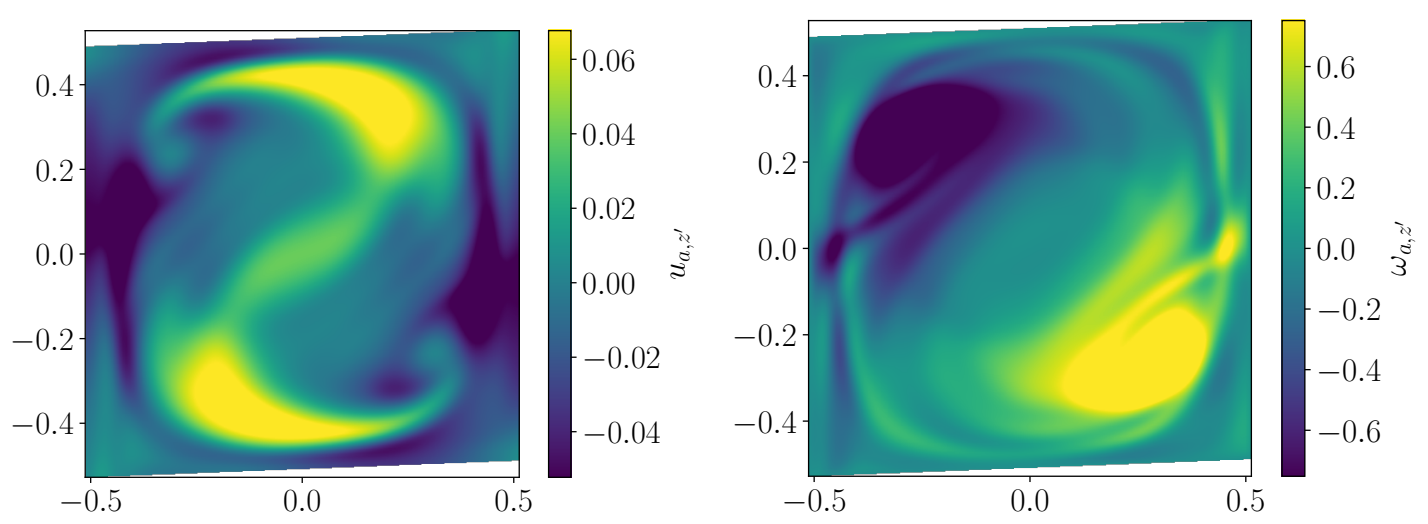

Figure 3.60: $u_{a, z^{\prime}}$ (left) and $\omega_{a, z^{\prime}}$ (right) for $\mathrm{Ek}=1.0 \cdot 10^{-4}$ and $\Omega_{P}=-0.008$ in the plane normal to $\boldsymbol{z}^{\prime}$ through the origin. A azimuthal 2-mode in $u_{a, z^{\prime}}$ and a 1 -mode in $\omega_{a, z^{\prime}}$ is visible. 


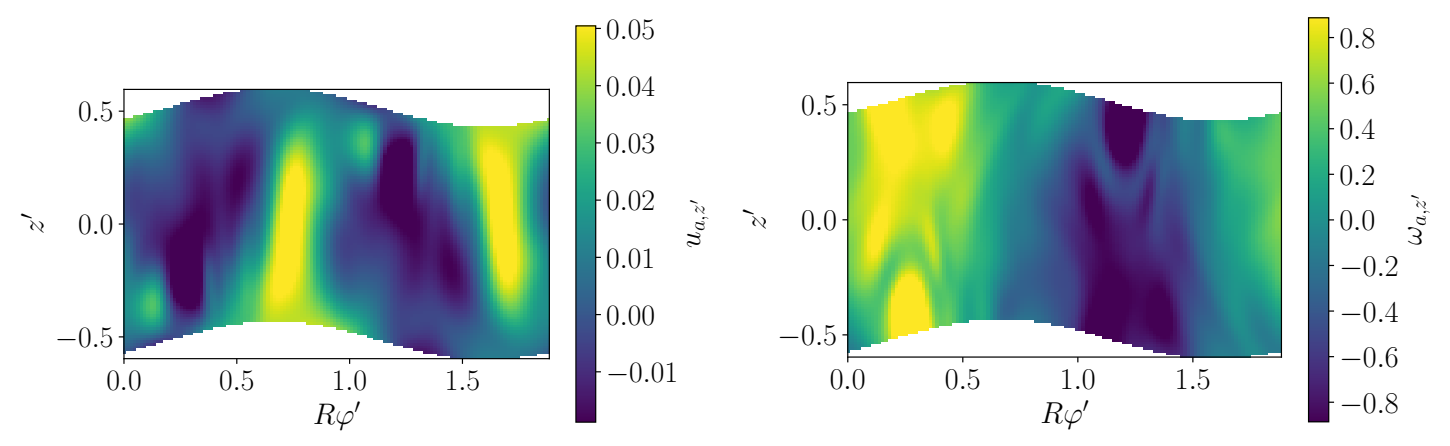

Figure 3.61: $u_{a, z^{\prime}}$ (left) and $\omega_{a, z^{\prime}}$ (right) for $\mathrm{Ek}=1.0 \cdot 10^{-4}$ and $\Omega_{P}=-0.008$ in the plane normal to $\boldsymbol{z}^{\prime}$ through the origin. A azimuthal 2-mode in $u_{a, z^{\prime}}$ and a 1 -mode in $\omega_{a, z^{\prime}}$ is visible.

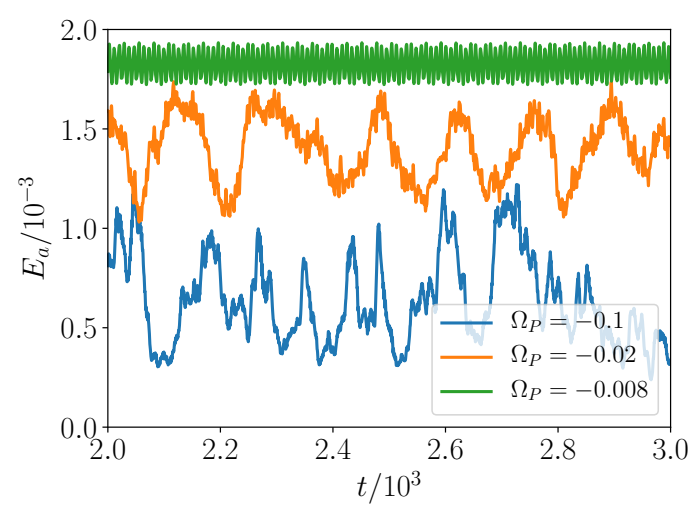

Figure 3.62: $E_{a}(t)$ at $\mathrm{Ek}=1.0 \cdot 10^{-4}$ devolves even faster into chaotic behavior than at $\mathrm{Ek}=2.5 \cdot 10^{-4}$ in fig. 3.58 and 3.59 .
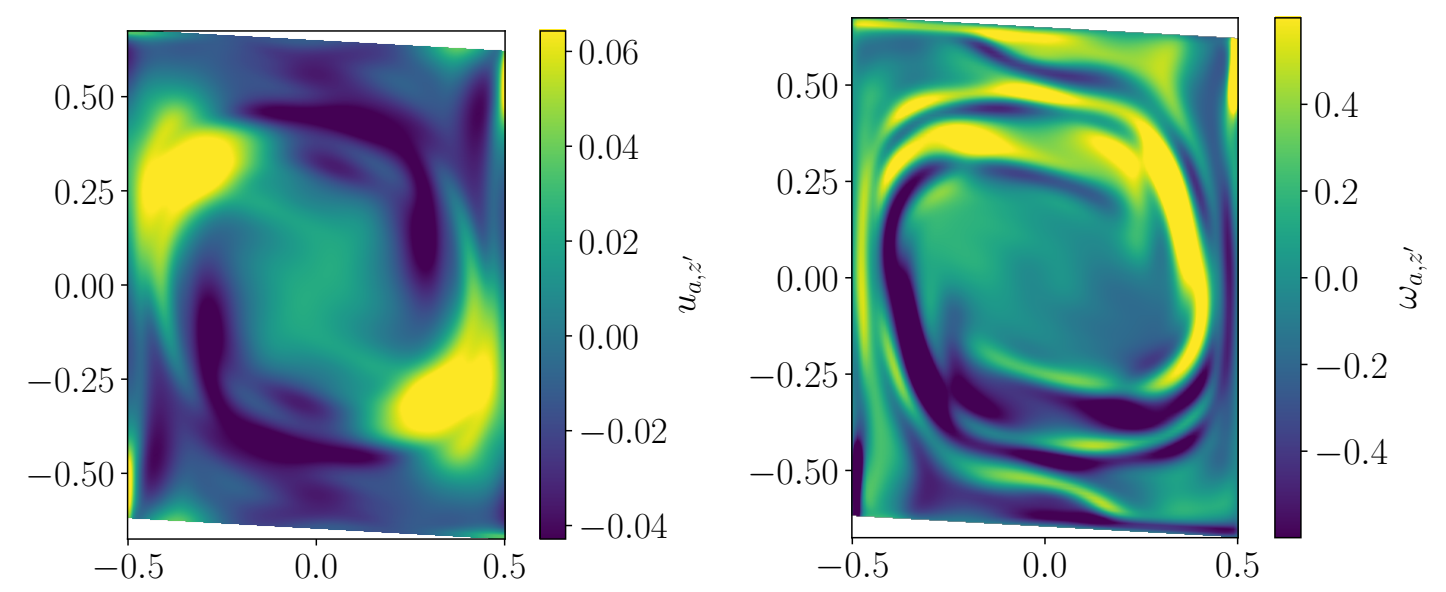

Figure 3.63: $u_{a, z^{\prime}}$ (left) and $\omega_{a, z^{\prime}}$ (right) for Ek $=1.0 \cdot 10^{-4}$ and $\Omega_{P}=-0.03$ in the plane normal to $\boldsymbol{z}^{\prime}$ through the origin. 

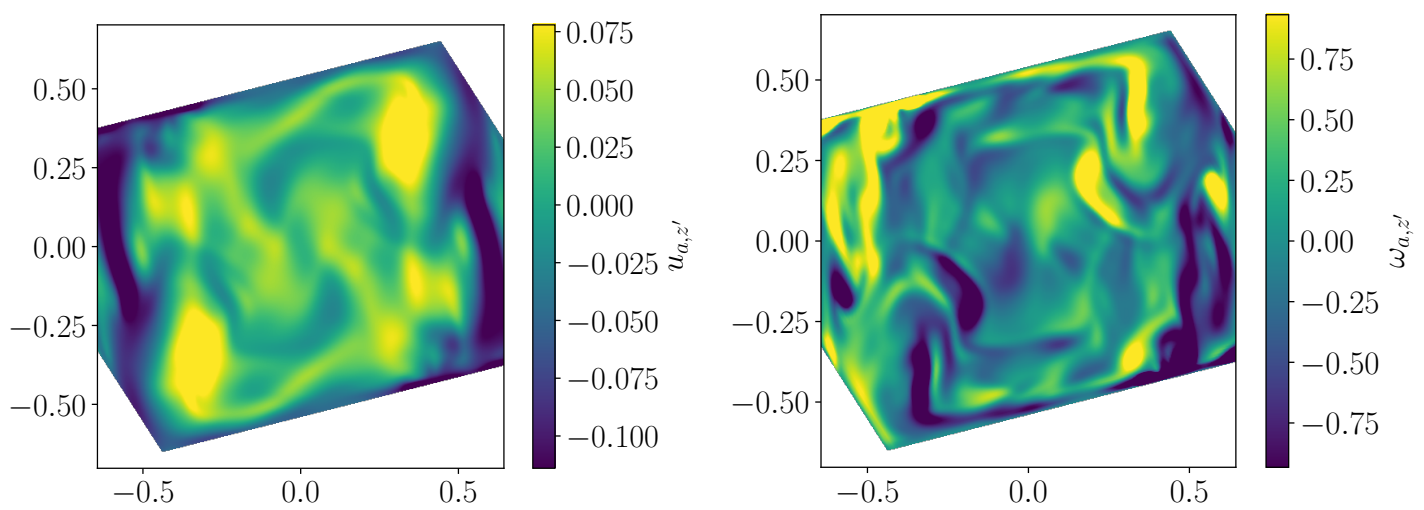

Figure 3.64: $u_{a, z^{\prime}}$ (left) and $\omega_{a, z^{\prime}}$ (right) for $\mathrm{Ek}=1.0 \cdot 10^{-4}$ and $\Omega_{P}=-0.04$ in the plane normal to $\boldsymbol{z}^{\prime}$ through the origin.
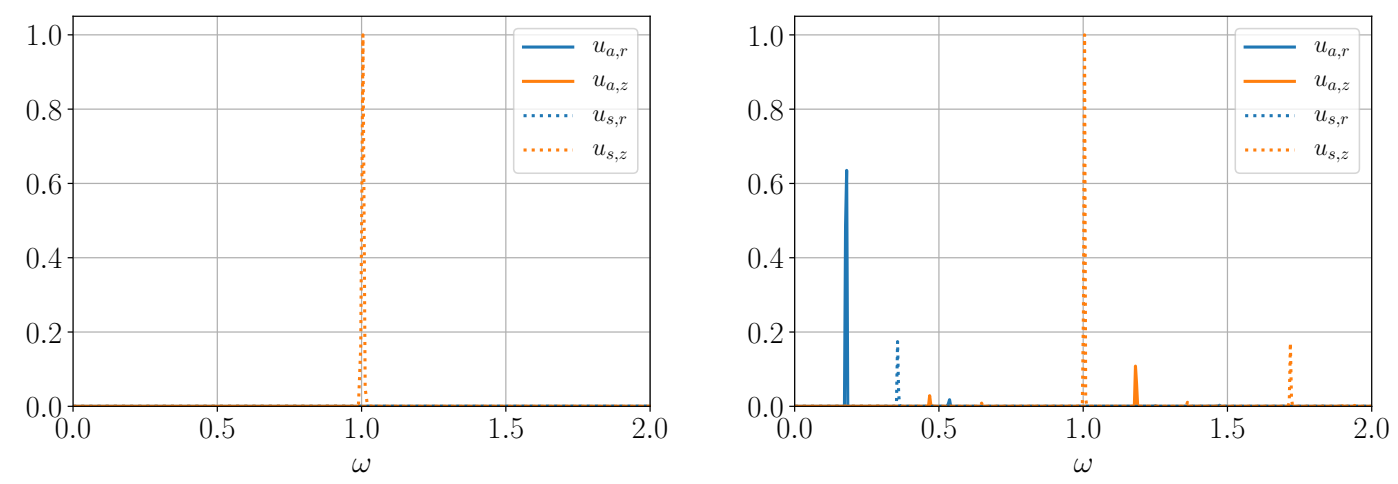

Figure 3.65: Power density spectrum of characteristic velocity components $u_{a, r}, u_{a, z}$, $u_{s, r}$ and $u_{s, z}$ at $\mathrm{Ek}=1.0 \cdot 10^{-4}$ and $\Omega_{P}=-0.008$ for the stable hysteresis branch with $E_{a}=0$ (left) and for the unstable branch with $E_{a} \neq 0$ (right), normalized to the peak of the spin-over mode. 

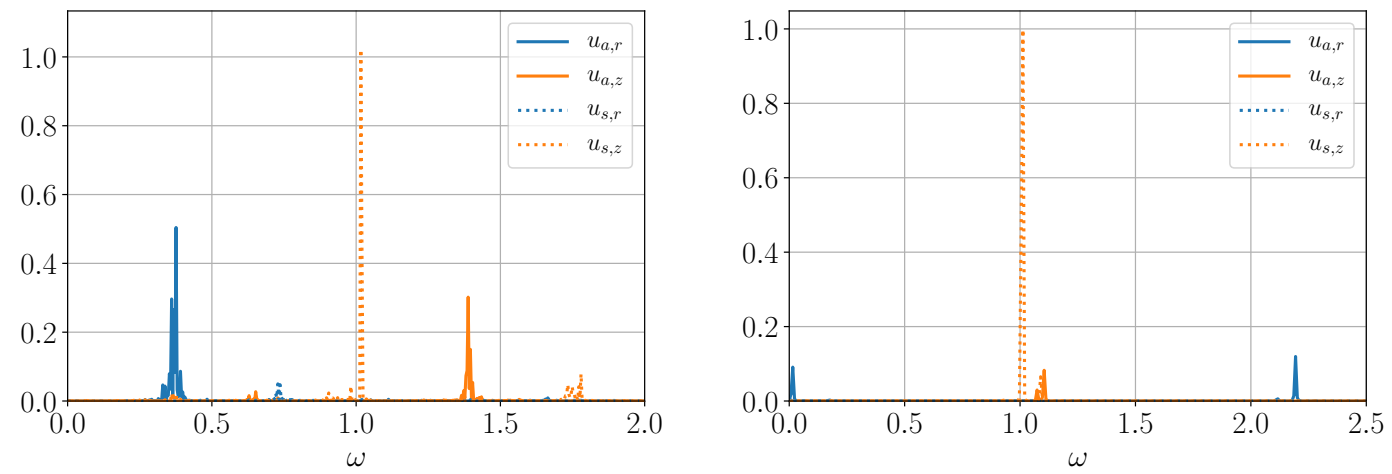

Figure 3.66: Power density spectrum of characteristic velocity components $u_{a, r}, u_{a, z}, u_{s, r}$ and $u_{s, z}$ at $\mathrm{Ek}=1.0 \cdot 10^{-4}$ and $\Omega_{P}=-0.035$ (left) and at $\mathrm{Ek}=2.5 \cdot 10^{-4}$ and $\Omega_{P}=-0.02$ (right), normalized to the peak of the spin-over mode.
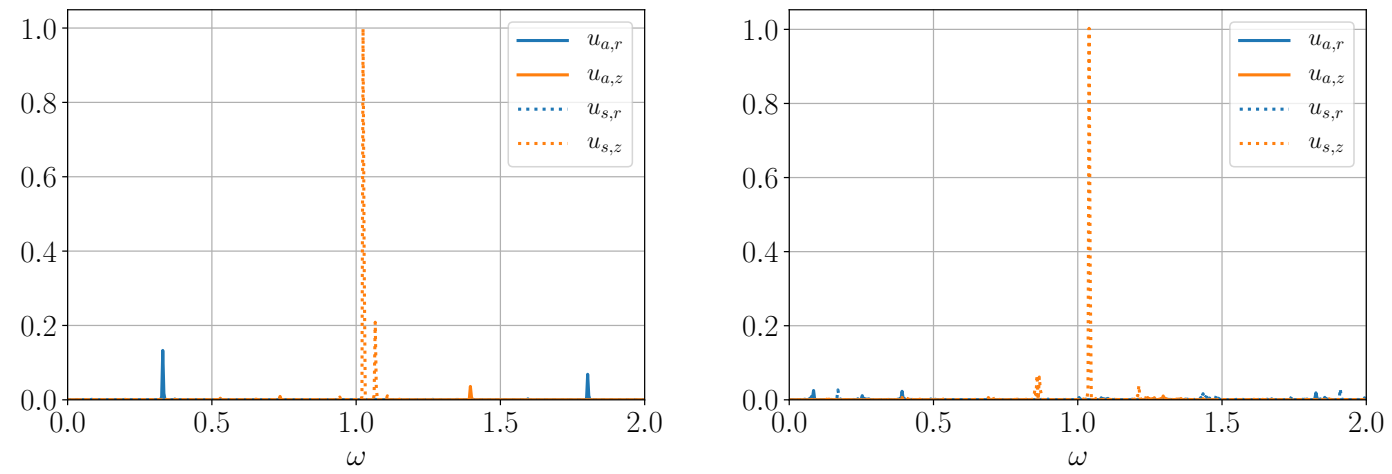

Figure 3.67: Power density spectrum of characteristic velocity components $u_{a, r}, u_{a, z}, u_{s, r}$ and $u_{s, z}$ at $\mathrm{Ek}=2.5 \cdot 10^{-5}$ and $\Omega_{P}=-0.05$ (left) and $\Omega_{P}=-0.08$ (right), normalized to the peak of the spin-over mode. 


\section{Kinematic Dynamo}

Up to this point of this chapter, all simulations were done for purely hydrodynamic cases, to examine the different flow structures and instabilities, which could possibly drive a dynamo effect inside the given geometry. This section will now address the suitability of the found flow patterns in section 3 for generating a magnetic field by using kinematic dynamos. To do this, the fully developed hydrodynamic simulations were taken and the magnetic field $\boldsymbol{B}$ added to the set of field parameters containing $\boldsymbol{u}$ and $\rho$. The change of the magnetic field over time is governed by the induction equation defined in 2.20. Additionally equations of motions described in section 2.1.1 and already used for the purely hydrodynamic flow will be solved simultaneously. For a full dynamo model, usually the Lorentz force $\boldsymbol{F}_{B}=(\boldsymbol{\nabla} \times \boldsymbol{B}) \times \boldsymbol{B}$ would be added to the Navier-Stokes equation of motion to incorporate the feedback of the magnetic field on the fluid, but this is not necessary to assess the possibility of generating a magnetic field. The starting point of magnetic field generation is of course a preexisting flow with a weak non-zero magnetic field for seeding. This field is set as $\boldsymbol{B}(t=0)=\left(B_{x}, B_{y}, B_{z}\right)=\left(10^{-8}, 10^{-8}, 10^{-8}\right)$. In the case that the flow is capable of sustaining the magnetic field and therefore dynamo action, the magnetic energy must not vanish, i.e.

$$
E_{B} \nrightarrow 0 \text { for } t \rightarrow \infty .
$$

The magnetic energy is defined as

$$
E_{B}=\frac{1}{2} \int \boldsymbol{B}^{2} \mathrm{~d} V
$$

In a simple understanding, this is achieved, if the magnetic field generated by the velocity field at least compensates for the ohmic dissipation. This process is dependent on the magnetic Prandtl number Pm in eq. 2.20, so that it is reasonable to look for a critical $\mathrm{Pm}_{c}$ of a known flow, for which the previous condition in eq. 4.1 is satisfied. The low values of the magnetic field at the start suggests, that the Lorentz force is negligible at this point and the growth will be independent of it. Since the Lorentz force works 
by definition as a force against its origin, neglecting it will remove this back reaction and the magnetic field will grow or decay further dependent on the suitability of the flow. While for a time independent flow the growth of $\boldsymbol{B}$ would be exponential, for time dependent flows this is not strictly the case. However, as long as the flow owns statistically time independent properties like in a balanced flow, an exponential growth can be used approximately, if the variation of the statistical properties of the flow over time is much smaller than the variation of the magnetic energy as can be seen in fig. 4.1. Here, an auxiliary fit function is defined:

$$
\hat{E}_{B}(t)=E_{B, 0} \mathrm{e}^{p t}
$$

The parameter $p$ characterizes the growth and $\mathrm{Pm}_{c}$ will be defined so that

$$
\operatorname{Pm}_{c}>\operatorname{Pm} \forall \operatorname{Pm} \text { with } p<0
$$

If $p>0$, i.e. the magnetic field grows, the full magnetohydrodynamic problem must be considered, as soon as the Lorentz force is not negligible anymore and information about the saturation of the field could be gained. However, the focus of this work is the initial problem of the possibility of field growth, so it is not investigated further. To asses $\mathrm{Pm}_{c}$ some simulations were ran with different Pm and eq. 4.3 fitted onto $E_{B}(t)$ and with help of the resulting growth rates $p(\mathrm{Pm}) p=0$ interpolated. This point marks $\mathrm{Pm}_{c}$. This was
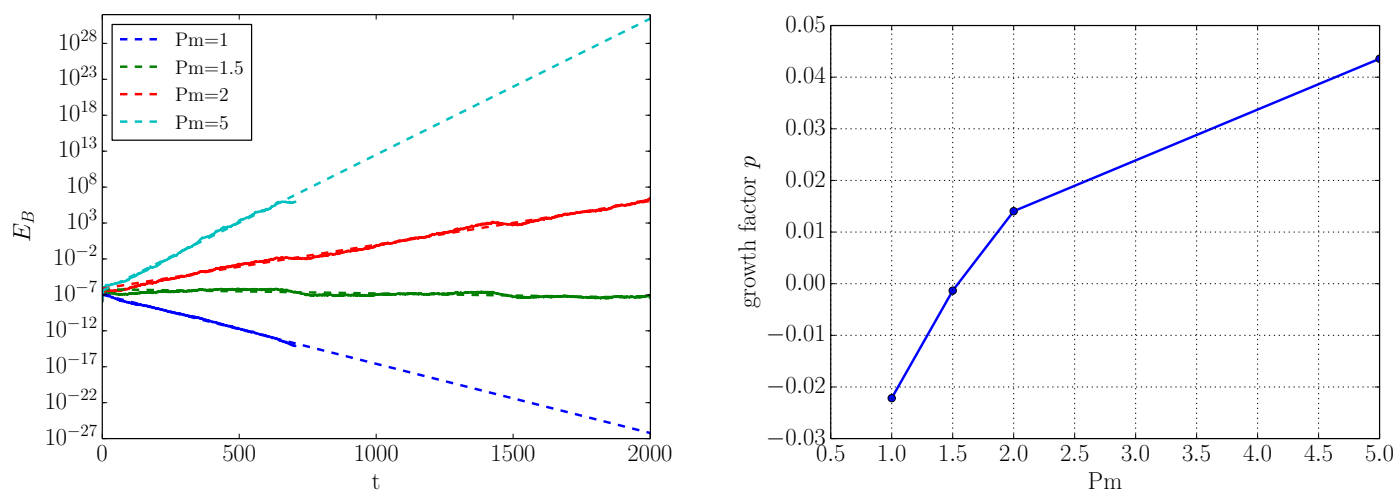

Figure 4.1: Time evolution of magnetic energy $E_{B}(t)$ (left) and growth factor $p$ (right) for different $\mathrm{Pm}$ at $\mathrm{Ek}=2.5 \cdot 10^{-4}$ and $\Omega_{P}=-0.16$

done for a broad spectrum of parameters, repurposing simulations done in 3, ranging from $\mathrm{Ek}=10^{-3}$ to $10^{-5}$ and from $\Omega_{P}=-0.3$ to $\Omega_{P}=-0.02$. Some results are pictured in fig. 4.2 for fixed $\Omega_{P}$. While one may notice some variations in the magnetic Prandtl number, it is clear, that, overall, it decreases with decreasing Ekman number. This may 
indicate a possible asymptotic behavior of the magnetic Reynolds number $\mathrm{Rm} \propto \frac{\mathrm{Pm}}{\mathrm{Ek}}$ towards a constant value, allowing to speculate about possible dynamos at even lower Ekman numbers.

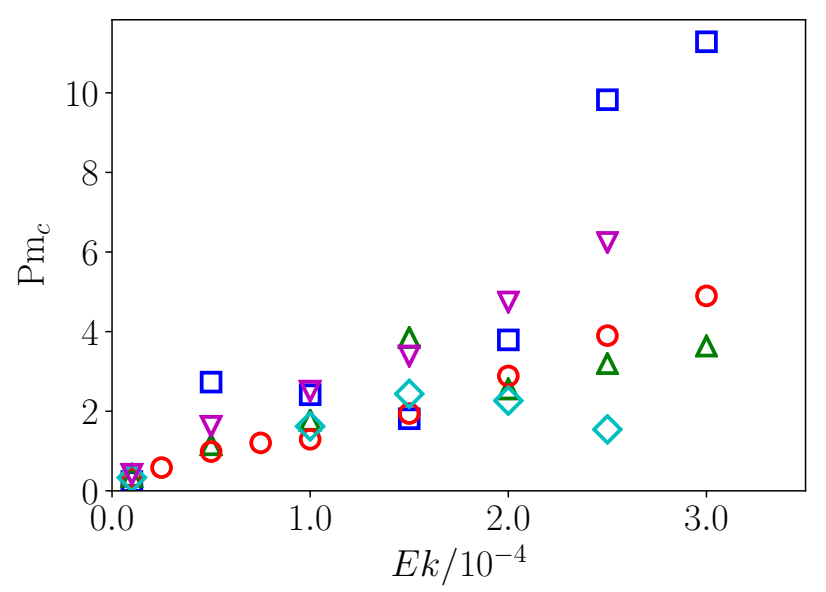

Figure 4.2: $\mathrm{Pm}_{c}(\mathrm{Ek})$ at $\Omega_{P}=-0.02$ (squares) $\Omega_{P}=-0.05$ (upward triangles), $\Omega_{P}=$ -0.1 (circles), $\Omega_{P}=-0.16$ (diamonds), $\Omega_{P}=-0.3$ (downward triangles) decreases with decreasing Ek.

\subsection{Antisymmetric Magnetic Reynolds Number}

First, an exact and useful definition of the magnetic Reynolds number is necessary. For the dynamos depending on the instability a characteristic number expressing the relevant motion is defined as

$$
\mathrm{Rm}_{c, a}=\sqrt{2 E_{a}} \frac{\mathrm{Pm}_{c}}{\mathrm{Ek}}
$$

Here, the typical velocity inside the general definition of $R m$, where $R m=\frac{u_{s} L_{s}}{\eta_{s}}$ is the average of the antisymmetric velocity, being the main contribution to triad resonances with $\bar{u}_{a}=\sqrt{2 E_{a}}$. The obvious advantages against total kinetic energy is the omission of the energy of the spin-over mode, which does not contribute to any dynamo as a simple two-dimensional solid-body rotation [Moffatt, 1978]. This is also valid under the boundary condition as it can be seen at $\mathrm{Ek}=2.5 \cdot 10^{-4}$ and $\Omega_{P}=-0.04$ with no identified instability. Here it was not possible to define a $\mathrm{Pm}_{c}$ since no $\mathrm{Pm}$ with positive $p$ was found for simulations run up to $\mathrm{Pm}=50$. So, by this definition, the lower $\mathrm{Rm}_{c, a}$ is, the better is the dynamo connected to the antisymmetric motion. Also, this 
definition is invariant to reference system transitions, it is unchanged in each mantle, precession or inertial frame. Since neither high $\left(\right.$ like $\left.\mathrm{Ek}=10^{-3}\right)$ nor low $\left(\mathrm{Ek}=10^{-5}\right)$ Ekman numbers showed any signs of the dynamo effect relying on a suitable instability, this measure works best for Ekman numbers like $\mathrm{Ek}=1.0 \cdot 10^{-4}$ and $\mathrm{Ek}=2.5 \cdot 10^{-4}$, where numerous simulations were done as pictured in fig 4.3 and prominent values for this and other magnetic Reynolds numbers are noted in tab. 4.1. The minimal critical number for $\mathrm{Ek}=1.0 \cdot 10^{-4}$ lies at $\Omega_{P}=-0.008$ with $\mathrm{Rm}_{\mathrm{c}, \mathrm{a}}=220$. The hydrodynamic flows this simulations were based upon were of course taken from the unstable branch in figure 3.51. The stable branch does not show any signs of notable dynamo effect, since for all simulated magnetic Prandtl numbers up to $\mathrm{Pm}=10$ the growth parameter $p$ is always smaller than zero. Here, the only identified mechanism possibly driving a dynamo is the $(1,2)$-triad resonance of section 3.5. The value worsens with increasing precession rate until $\mathrm{Rm}_{\mathrm{c}, \mathrm{a}}=1938$ at $\Omega_{P}=-0.03$, presumably connected to the decay of the triad resonance. For $\Omega_{P}<-0.03$ the antisymmetric magnetic Reynolds number is always lower than 650 , however, other effects like the $s_{2} t_{1}$ from section 3.4 become notable and do not rely on the antisymmetric motion, so $\mathrm{Rm}_{\mathrm{c}, \mathrm{a}}$ may not be conclusive anymore. Similar problems exist for $\mathrm{Ek}=2.5 \cdot 10^{-4}$ with non-antisymmetric effects at $\Omega_{P}<-0.08$. For lower precession rates, no other effect as triad resonances were identified, so here it may be a good measure further on. For the best $\mathrm{Rm}_{\mathrm{c}, \mathrm{a}}$ of 155 at $\Omega_{P}=-0.045$ again the main mechanism is the $(1,2)$-triad. It is even lower than the minimum at $\Omega_{P}>-0.08$, since the antisymmetric energy is much lower $\left(E_{a}(\mathrm{Ek}=\right.$ $\left.\left.2.5 \cdot 10^{-4}, \Omega_{P}=-0.02\right)=6.26 \cdot 10^{-4} E_{a}\left(\mathrm{Ek}=1.0 \cdot 10^{-4}, \Omega_{P}=-0.008\right)=1.82 \cdot 10^{-3}\right)$, having a large impact on the magnetic Reynolds number. At $\Omega_{P}=-0.02$, the main contribution to the hydrodynamic flow was the $(4,5)$-triad, which does not support the dynamo as efficiently as the other triad, with $\mathrm{Rm}_{\mathrm{c}, \mathrm{a}}=804$.

\subsection{Full-Flow Magnetic Reynolds Number}

A more practical approach is to base the magnetic Reynolds number on kinetic energy $E_{k i n}$. The full active flow is considered without neglecting effects with no antisymmetric part:

$$
\mathrm{Rm}_{c, k i n}=\sqrt{2 E_{k i n}} \frac{\mathrm{Pm}_{c}}{\mathrm{Ek}}=\sqrt{\frac{E_{k i n}}{E_{a}}} \mathrm{Rm}_{c, a} .
$$

Since $E_{k i n}$ is a direct measure for motion relative to the boundaries, the dissipation will increase proportional to $E_{k i n}$, although the exact functional dependence is unknown. 


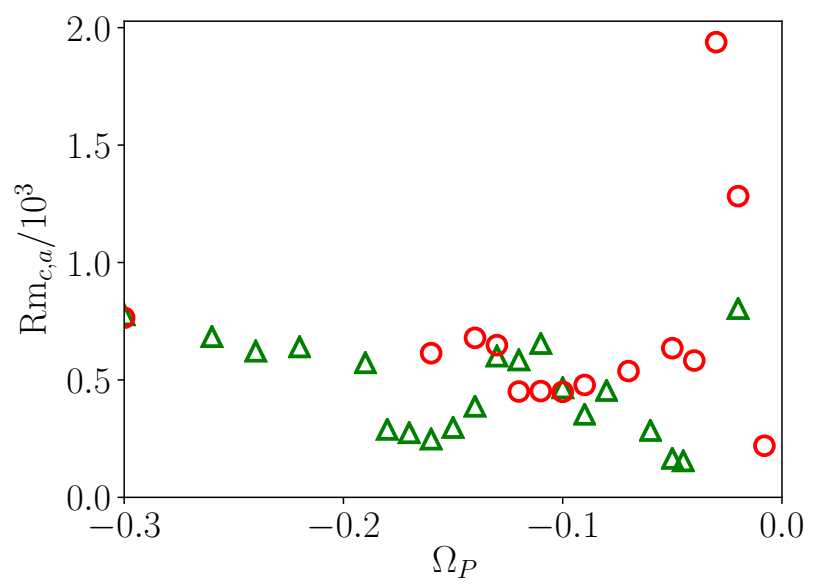

Figure 4.3: $\mathrm{Rm}_{\mathrm{c}, \mathrm{a}}$ for $\mathrm{Ek}=1.0 \cdot 10^{-4}$ (circles) and $\mathrm{Ek}=2.5 \cdot 10^{-4}$ (triangles): For $\mathrm{Ek}=$ $1.0 \cdot 10^{-4}$ and $\Omega_{P}>=-0.02$ hydrodynamic simulations of the unstable branch were taken.

This is an important notion for designing laboratory experiments: One of the main restrictions is the maximal power motors deliver to drive the experiment and therefore a low dissipation rate is preferable. With this in mind, minimizing $\mathrm{Rm}_{c, k i n}$ is a good starting point for practical approaches. The inclusion of all motion into the magnetic Reynolds number makes it more informative for effects that do not break the centrosymmetry and so it is reasonable to investigate dynamos at other Ekman number than while considering $\mathrm{Rm}_{\mathrm{c}, \mathrm{a}}$. The results are presented in fig. 4.4. On average $\mathrm{Rm}_{c, k i n}$ must be higher than $\mathrm{Rm}_{c, a}$, since $E_{a}$ is roughly two orders of magnitude lower than $E_{k i n}$ for most runs (compare fig. 3.51 and fig. 3.4). For $\mathrm{Ek}=2.5 \cdot 10^{-4}$ and $\mathrm{Ek}=1.0 \cdot 10^{-4}$ the resulting values of $\mathrm{Rm}_{c, k i n}$ are qualitatively similar to those of $\mathrm{Rm}_{c, a}$ and even here the minimum at $\Omega_{P}=-0.008$ and $\mathrm{Ek}=1.0 \cdot 10^{-4}$ is again the lowest possible value with $\mathrm{Rm}_{c, k i n}=537$. The bad ratio of $\frac{E_{a}}{E_{k i n}}$ at $\mathrm{Ek}=2.5 \cdot 10^{-4}$ and $\Omega_{P}=-0.045$ on the other hand neutralizes this minimum. With no instability at $\Omega_{P}=-0.04$, it was not possible to achieve any reasonable $\mathrm{Pm}_{c}$ below 50, indicating that the flow in this region is not able to support a dynamo without an instability. Since no other effect than the spin-over mode is at work at these parameters, this is the expected behaviour. $\Omega_{P}=-0.16$ or, respectively, the area from $\Omega_{P}=-0.18$ to -0.14 is minimal for $\mathrm{Rm}_{c, k i n}$ down to 2135 , giving significance to the strong and stable $s_{2} t_{1}$-mode described in section 3.4. The oscillation of these modes for $\Omega_{P}=-0.13$ to -0.09 suppress the dynamo effect and the critical values lie significantly above that with stable axisymmetric modes. Similar, around $\Omega_{P}=-0.12$ 


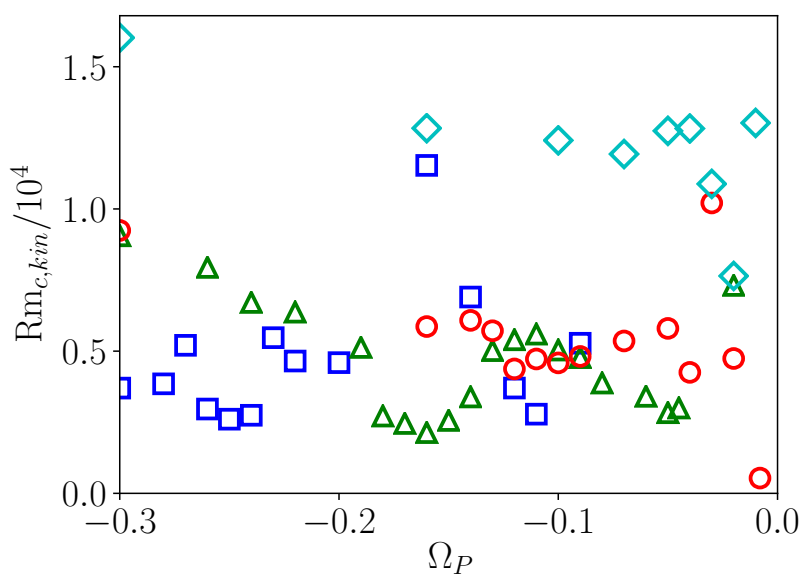

Figure 4.4: $\mathrm{Rm}_{\mathrm{c}, \mathrm{kin}}$ for $\mathrm{Ek}=1.0 \cdot 10^{-3}$ (squares), $\mathrm{Ek}=2.5 \cdot 10^{-4}$ (triangles), $\mathrm{Ek}=$ $1.0 \cdot 10^{-4}$ (circles) and $\mathrm{Ek}=1.0 \cdot 10^{-5}$ (diamonds).

to -0.05 at $\mathrm{Ek}=1.0 \cdot 10^{-4}$ the axisymmetric modes oscillate but $\mathrm{Rm}_{c, k i n}$ decreases weakly, while the mean energy value of the oscillating $s_{2} t_{1}$-mode gains notable size (see fig. 3.49 and fig. 3.37). The breakdown for $\Omega_{P} \leq-0.13$ show again an increase in the critical Full-Flow Magnetic Reynolds Number revealing a weak dynamo effect even for the oscillating mode in contrast to the more chaotic breakdown regime. At the relatively high $\mathrm{Ek}=1.0 \cdot 10^{-3}$ the flow shows no time dependent instability for any tested $\Omega_{P}$. Nevertheless it does support dynamo effects on a similar scale as at lower Ekman numbers, even at points like $\Omega_{P}=-0.2$ with only small contribution of $s_{1} t_{1}$-mode and no sign of a vortex. This may be the result of shearing effects at the boundaries. This is especially notable at $\Omega_{P}=-0.25$ with a minimum of $\mathrm{Rm}_{c, k i n}=2608$. Here, the strong tilt of the fluid axis in fig. 3.9 generates strong shearing in the corners, as can be seen in the visualization of vorticity in fig. 4.5 and the adherence of the growing magnetic field ( with $\mathrm{Pm}=10$ ) to it. The $s_{2} t_{1}$-mode in this region is too weak to affect any dynamo effect significantly (see fig. 3.37 and fig. 3.49). The region from $\Omega_{P}=-0.18$ to -0.11 is characterized by an antisymmetric (with regard to the centrosymmetry) displacement noted in section 3.2 and a combination of $s_{1} t_{1}$ - and $s_{2} t_{1}$-modes. The mechanisms behind this does not seem to help sustaining a magnetic field or even preventing it since $\mathrm{Rm}_{c, k i n}$ shows a sharp increase with increasing $\Gamma_{0}$, from $\mathrm{Rm}_{c, k i n}=2784$ at $\Omega_{P}=-0.11$ to 11531 at $\Omega_{P}=-0.16$ and no quantifiable critical number at $\Omega_{P}=-0.18$. At Ek $=10^{-5}$ on the other hand, no discernible triad resonances or $s_{1} t_{1}$ are left, the flow is mostly dominated by fine-scale turbulent behavior or the vortex region of section 3.3. The minimum 

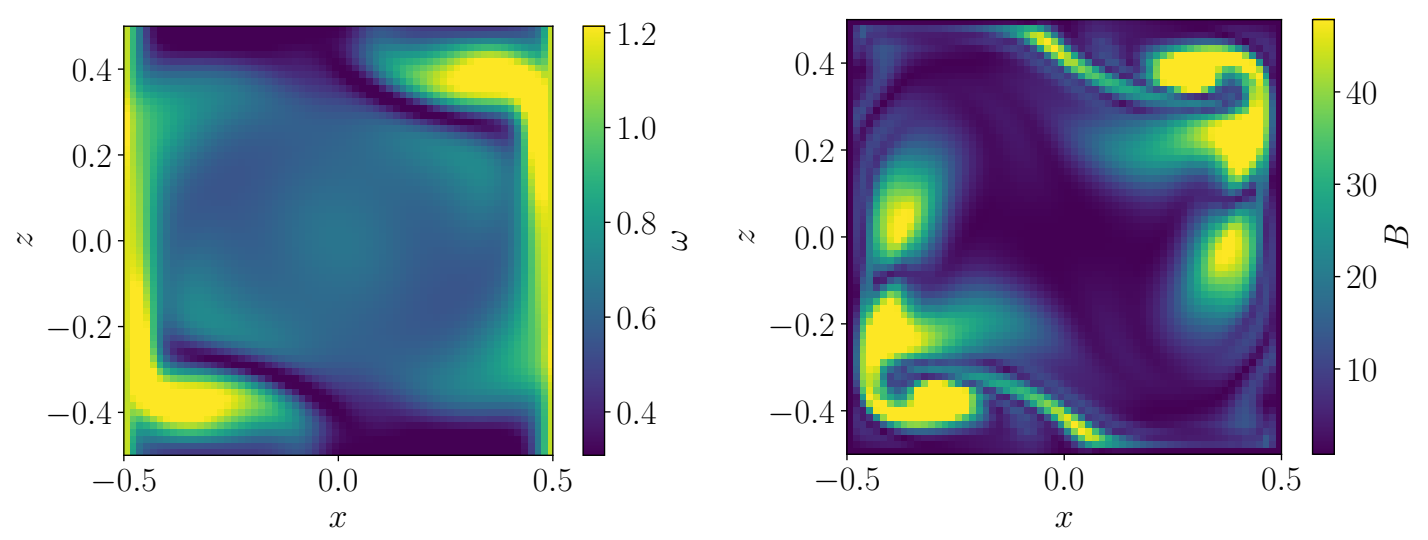

Figure 4.5: Vorticity $\omega$ (left) and magnetic field $B$ (right) at Ek $=1.0 \cdot 10^{-3}$ and $\Omega_{P}=$ -0.25 with $\mathrm{Pm}=10$.
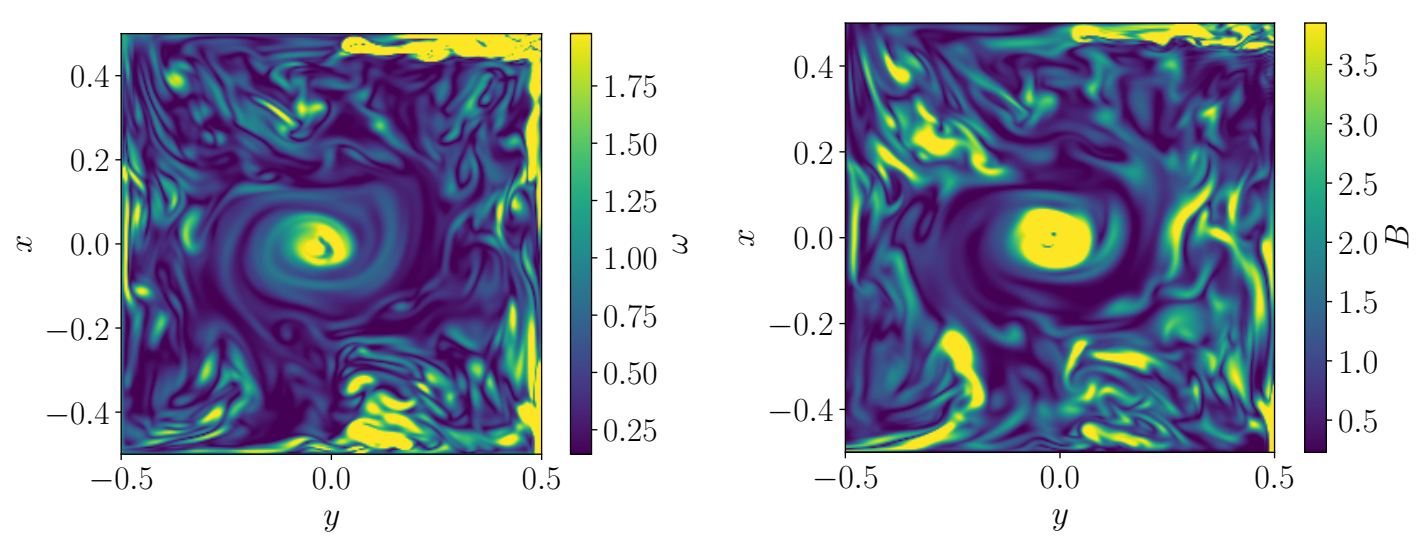

Figure 4.6: Vorticity $\omega$ (left) and magnetic field $B$ (right) at Ek $=1.0 \cdot 10^{-5}$ and $\Omega_{P}=$ -0.02 with $\mathrm{Pm}=0.3$.

$\mathrm{Rm}_{c, \text { kin }}=7642$ at $\Omega_{P}=-0.02$ lies within the region vortex and with no other significant process for supporting a dynamo, it can be assumed as the cause. Similar reasoning applies to $\Omega_{P}=-0.03$ with $\mathrm{Rm}_{c, k i n}=10880$, while $\mathrm{Rm}_{c, k i n} \approx 12500$ is usually seen at other values. The $\mathrm{Rm}_{c, k i n}$ highest precession rate at $\Omega_{P}=-0.3$ is even set at 16020 . See tab. 4.1 for a conclusion of all results.

\subsection{Rotational Magnetic Reynolds Number}

A third, more simple definition of a magnetic Reynolds number drops the use of direct fluid motion to evaluate the characteristic velocity and concentrates on the more easily 
controllable rotation speed of the container, instead: The velocity the mantle rotates with can be assumed as $u=\left(\widetilde{\omega}_{D}+\widetilde{\Omega}_{P} \cos \alpha\right) \frac{L}{2}$. The term $\left(\widetilde{\omega}_{D}+\widetilde{\Omega}_{P} \cos \alpha\right)$ acknowledges the overall rotation around the rotation axis, $\frac{L}{2}$ is the distance of the wall from it. If the general definition of magnetic Reynolds number is $\mathrm{Rm}=\frac{u_{s} L_{s}}{\eta_{s}}$, the rotational magnetic Reynolds number can be expressed by non-dimensionalized quantities as

$$
\operatorname{Rm}_{c, r o t}=\frac{\mathrm{Pm}_{c}}{2 \mathrm{Ek}}
$$

The results are presented in fig. 4.7 with the key results in tab. 4.1. Relative to

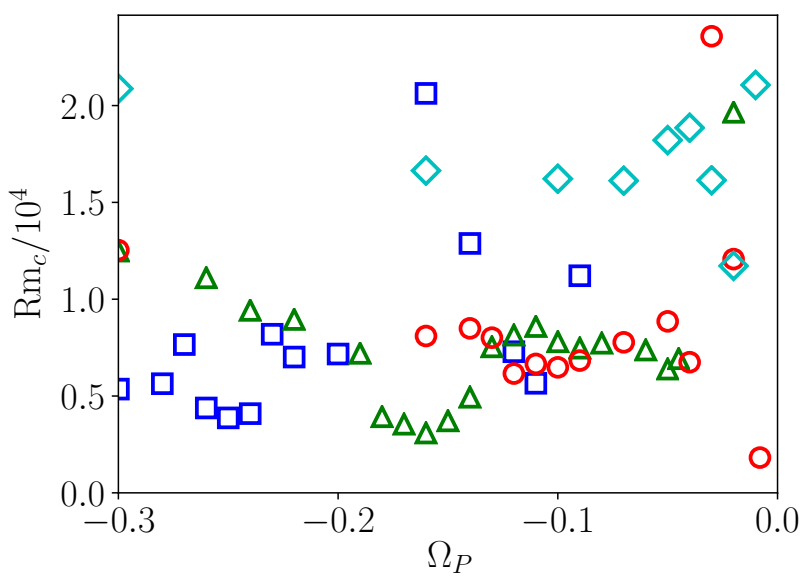

Figure 4.7: $\mathrm{Rm}_{\mathrm{c}, \text { rot }}$ for $\mathrm{Ek}=1.0 \cdot 10^{-3}$ (squares), $\mathrm{Ek}=2.5 \cdot 10^{-4}$ (triangles), $\mathrm{Ek}=$ $1.0 \cdot 10^{-4}$ (circles) and $\mathrm{Ek}=1.0 \cdot 10^{-5}$ (diamonds).

$\mathrm{Rm}_{c, k i n}$, the characteristic points are the same, with the respective minima $\mathrm{Rm}_{c, \text { rot }}=$ 3087 for $\Omega_{P}=-0.16$ and $\mathrm{Ek}=2.5 \cdot 10^{-4}, \mathrm{Rm}_{c, r o t}=1820$ for $\Omega_{P}=-0.008$ and $\mathrm{Ek}=1.0 \cdot 10^{-4}, \mathrm{Rm}_{c, \text { rot }}=3871$ for $\Omega_{P}=-0.25$ and $\mathrm{Ek}=1.0 \cdot 10^{-3}$ and $\mathrm{Rm}_{c, \text { rot }}=$ 11721 for $\Omega_{P}=-0.02$ and $\mathrm{Ek}=1.0 \cdot 10^{-5}$. If this is compared to the setup and parameters used in the experiment DresDYN, $\mathrm{Rm}_{\text {rot }}$ is limited to values below 1420 [Stefani et al., 2012, Stefani et al., 2015], being smaller than any of the values done in this work. However, the experiment will run at $\mathrm{Ek} \approx 10^{-8}$, three magnitudes below what could be simulated with sufficient resolution and in timely fashion. Therefore, extrapolations from known data to lower Ekman numbers is necessary at this point. Overall, $\mathrm{Rm}_{c, k i n}$ at $\mathrm{Ek}=10^{-5}$ is arguably high relative to higher Ekman numbers, contradicting the assumption earlier, that it may show asymptotic behavior towards low Ekman numbers. This becomes much clearer when examining $\mathrm{Rm}_{c, \text { rot }}$ at fixed $\Omega_{P}$ like in fig. 4.8: At first, the investigation of the variation of $\mathrm{Rm}_{c, \text { rot }}$ is complicated, since 
the flow can undergo different transitions between different states, as triad resonances, single vortex states and axisymmetric motions appear and vanish while lowering Ek. This is particular apparent at $\Omega_{P}=-0.02$ with a strong varying $\mathrm{Rm}_{c, \text { rot }}$ over all Ek. For higher precession rates like $\Omega_{P}=-0.1$ and $\Omega_{P}=-0.3$ this effect diminishes with the lower rate of transitions that occur. At $\Omega_{P}=-0.3$ the flow is always small-scale turbulent for the considered range from $\mathrm{Ek}=3.0 \cdot 10^{-4}$ to $\mathrm{Ek}=1.0 \cdot 10^{-5}$. The flows critical value decreases until $\mathrm{Ek} \approx 1.0 \cdot 10^{-4}$, after that, $\mathrm{Rm}_{c, \text { rot }}$ increases again linearly with $\Omega_{P}$.

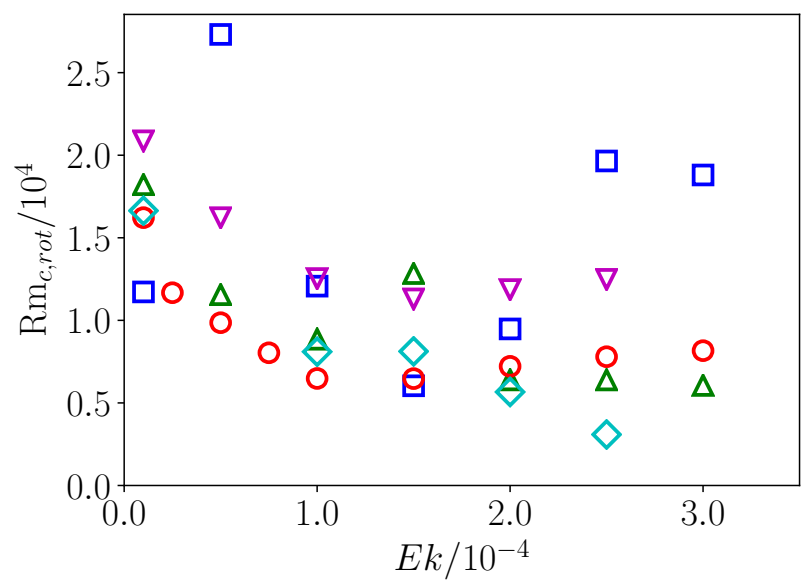

Figure 4.8: $\mathrm{Rm}_{\mathrm{c}, \text { rot }}$ for $\Omega_{P}=-0.02$ (squares), $\Omega_{P}=-0.05$ (upward triangles), $\Omega_{P}=$ -0.1 (circles), $\Omega_{P}=-0.16$ (diamonds) and $\Omega_{P}=-0.3$ (downward triangles)

Since no previously mentioned effect of flow structure correlates with this behavior, another explanation is more likely: the increasing turbulence and eddy diffusivity of low Ekman flows hamper the dynamo effect independently of the remaining flow structure. To examine this, a dissipation length scale is defined:

$$
L_{D}=\sqrt{\frac{E_{k i n}}{D}} .
$$

Here, $D$ is the dissipation that is given by the scalar product of 2.18 and $u$, resulting in:

$$
D=\frac{\mathrm{Ek}}{V}\left\langle\int \sum_{i j}\left(\delta_{i} u_{j}\right)^{2}\right\rangle=\frac{1}{V}\left\langle\boldsymbol{u} \cdot\left(\Omega_{P} \omega_{D}(\hat{\boldsymbol{z}} \times \boldsymbol{p}(t)) \times \boldsymbol{r}\right) \mathrm{d} V\right\rangle .
$$

Of course, both expressions can be used to calculate $u$, but the reliance of the former one on derivatives causes larger numerical errors when calculating $D$ from numerical 
results. Because of this, the latter expression was always used. Looking into fig. 4.9 displaying $L_{D}$ against Ek for fixed $\Omega_{P}$, one notices $L_{D}$ is relatively constant for high Ek and decreases sharply for low Ek. The influence on the magnetic Reynolds number is more significant, if it is plotted against $L_{D}$ as it is in fig. 4.10. The linear relation for fixed $\Omega_{P}$ is apparent, supporting the previously made assumption, that small scale motion in form of turbulence may increase the magnetic Reynolds number.

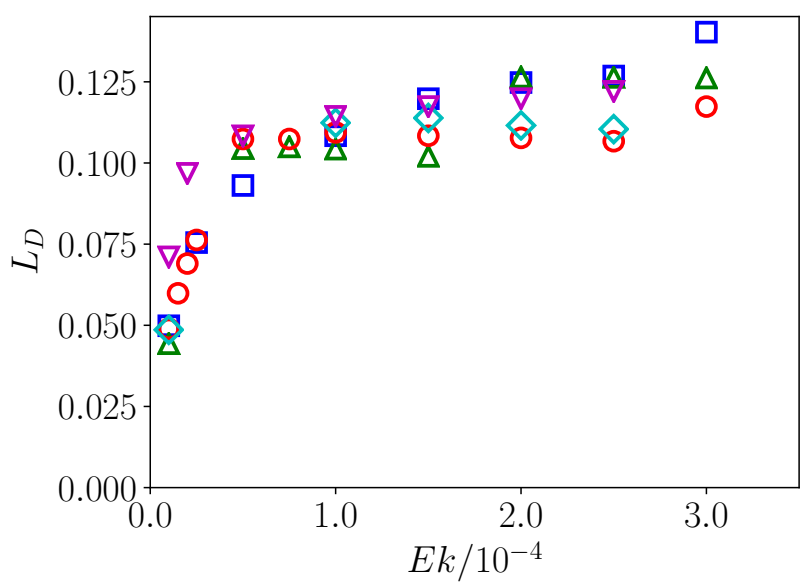

Figure 4.9: $L_{D}$ against $\mathrm{Ek}$ for $\Omega_{P}=-0.02$ (squares), $\Omega_{P}=-0.05$ (upward triangles), $\Omega_{P}=-0.1$ (circles), $\Omega_{P}=-0.16$ (diamonds) and $\Omega_{P}=-0.3$ (downward triangles). 


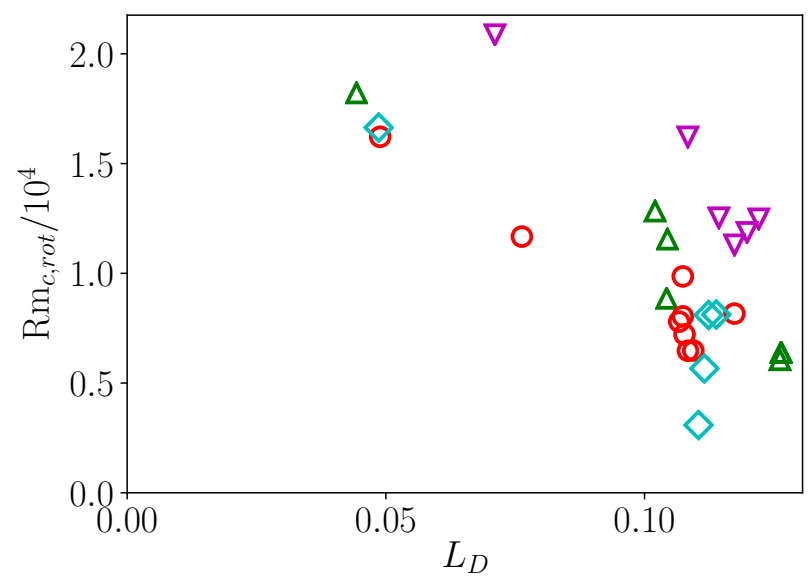

Figure 4.10: $\mathrm{Rm}_{\mathrm{c}, \text { rot }}$ against $L_{D}$ for $\Omega_{P}=-0.02$ (squares), $\Omega_{P}=-0.05$ (upward triangles), $\Omega_{P}=-0.1$ (circles), $\Omega_{P}=-0.16$ (diamonds) and $\Omega_{P}=-0.3$ (downward triangles).

\begin{tabular}{ll|cc|rrrr}
$\mathrm{Ek}$ & $\Omega_{P}$ & $E_{k i n}$ & $E_{a}$ & $\mathrm{Pm}_{c}$ & $\mathrm{Rm}_{c, a}$ & $\mathrm{Rm}_{c, k i n}$ & $\mathrm{Rm}_{c, r o t}$ \\
\hline $2.5 \cdot 10^{-4}$ & -0.02 & $1.73 \cdot 10^{-2}$ & $2.09 \cdot 10^{-4}$ & 9.83 & 804 & 7310 & 19652 \\
& -0.045 & $2.35 \cdot 10^{-2}$ & $6.26 \cdot 10^{-4}$ & 3.46 & 155 & 2999 & 6924 \\
& -0.16 & $5.98 \cdot 10^{-2}$ & $8.04 \cdot 10^{-2}$ & 1.54 & 248 & 2135 & 3087 \\
& -0.3 & $6.58 \cdot 10^{-2}$ & $4.84 \cdot 10^{-4}$ & 6.24 & 777 & 9055 & 12483 \\
\hline $1.0 \cdot 10^{-4}$ & -0.008 & $1.09 \cdot 10^{-2}$ & $1.82 \cdot 10^{-3}$ & 0.36 & 220 & 537 & 1820 \\
& -0.03 & $2.34 \cdot 10^{-2}$ & $8.45 \cdot 10^{-4}$ & 4.72 & 1938 & 10210 & 23578 \\
& -0.12 & $6.30 \cdot 10^{-2}$ & $6.68 \cdot 10^{-4}$ & 1.23 & 451 & 4375 & 6161 \\
& -0.3 & $6.80 \cdot 10^{-2}$ & $4.65 \cdot 10^{-4}$ & 2.51 & 765 & 9243 & 12532 \\
\hline $1.0 \cdot 10^{-5}$ & -0.02 & $5.31 \cdot 10^{-2}$ & $5.39 \cdot 10^{-4}$ & 0.23 & 769 & 7643 & 11721 \\
& -0.3 & $7.36 \cdot 10^{-2}$ & $2.06 \cdot 10^{-4}$ & 0.42 & 848 & 16020 & 20873
\end{tabular}

Table 4.1: Prominent sets of values of different definitions of magnetic Reynolds number. 


\section{Cuboids}

Up until now, this work only examined cubic systems in analogy to the default configuration of DRESDyn with a cylinder of same height as diameter. Expanding the focus to cuboids can be rewarding in hindsight of accessing already known instabilities but of other strength and serve as an analogy of already broadly examined[Wu and Roberts, 2011] ellipsoids. In the following section, two different cuboids were investigated to determine the effect of the instabilities in these based on their differing value of $E_{a} / E_{k i n}$.

\subsection{Hydrodynamics}

One form to describe an ellipsoid in standardized coordinates is

$$
x^{2}+y^{2}+(1+\eta) z^{2}=1
$$

with $\eta$ describing the oblateness of the object. Therefor, the axis length corresponding to $z$ is

$$
L_{Z}=(1-\eta)^{-0.5}
$$

while the other two axes stay $L_{X}=L_{Y}=1$. For the following used cuboids, these are used as edge lengths imitating the form of an ellipsoid like the cube did for a sphere. The

$$
\begin{array}{c|ccc}
\eta & 0.0 & -0.5 & -0.7 \\
\left(L_{X}, L_{Y}, L_{Z}\right) & (1,1,1) & (1,1,1.41) & (1,1,1.83)
\end{array}
$$

Table 5.1: The used values of $\eta$ and the translation into edge lengths of cuboids.

focus here is on three different values of $\eta, 0.0$ (as the cube for reference), -0.5 and -0.7 with the corresponding edge lengths in tab. 5.1. At a fixed value of $\mathrm{Ek}=2.5 \ldots 10^{-4}$, the variation of kinetic energy $E_{k i n}$ over $\Omega_{P}$ is quite similar to each other for $\eta=-0.7$ and $\eta=-0.5$ with the cube exceeding both at every precession rate (see fig. 5.1). Also, 


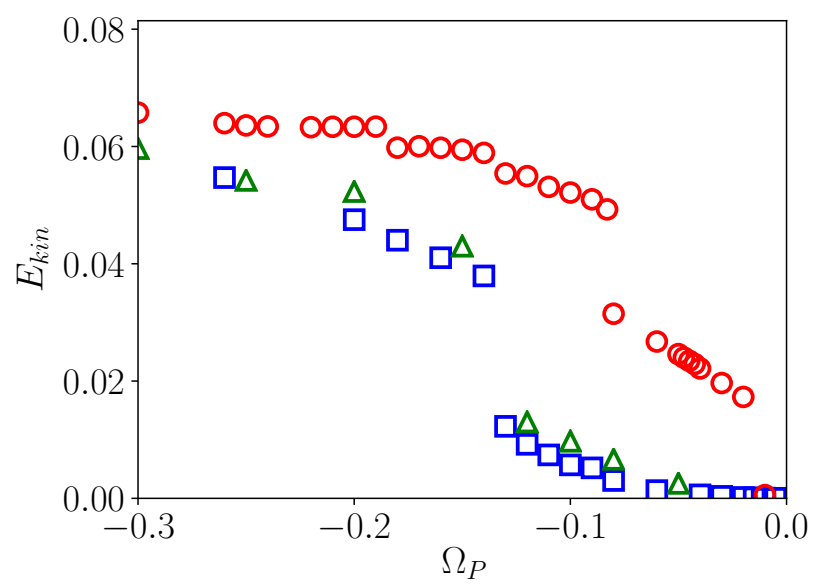

Figure 5.1: $E_{k i n}$ at $\mathrm{Ek}=2.5 \cdot 10^{-4}$ for $\eta=-0.7$ (squares), $\eta=-0.5$ (triangles), $\eta=$ 0.0 (circles)

while all of them experience the leap in kinetic energy as from sec. 3.1.1, the cube does it at a much smaller perturbation by precession. The antisymmetric energy $E_{a}$ as defined

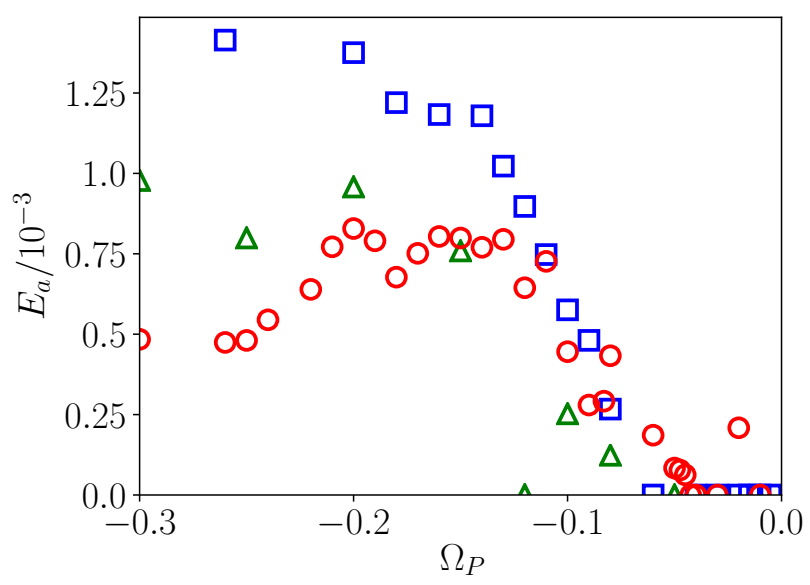

Figure 5.2: $E_{a}$ at $\mathrm{Ek}=2.5 \cdot 10^{-4}$ for $\eta=-0.7$ (squares), $\eta=-0.5$ (triangles), $\eta=$ 0.0 (circles)

in eq. 3.20 and pictured for $\mathrm{Ek}=2.5 \cdot 10^{-4}$ in fig. 5.2 gets the largest magnitude for $\eta=-0.7$. For $\eta=-0.5$ it is comparable to the cube $(\eta=0.0)$ in magnitude, but is split into two different regions at $\Omega_{P}=-0.12$. This indicates different causes for the instability for $\Omega_{P}>-0.12$ and for $\Omega_{P}<-0.12$. For $\eta=-0.7 E_{a}$ exceeds at higher precession rates in comparison to $\eta=0.0$ and $\eta=-0.5$ and in combination with the 
low value of $E_{k i n}$ in fig. 5.1, the ratio $\frac{E_{a}}{E_{k i n}}$ between them reaches 0.1 at $\Omega_{P}=-0.1$ as can be seen in 5.3, while it keeps below 0.04 for both of the other container geometries. The antisymmetric velocity at $\Omega_{P}=-0.1$ remains in form of a triad resonance (fig. 5.4)

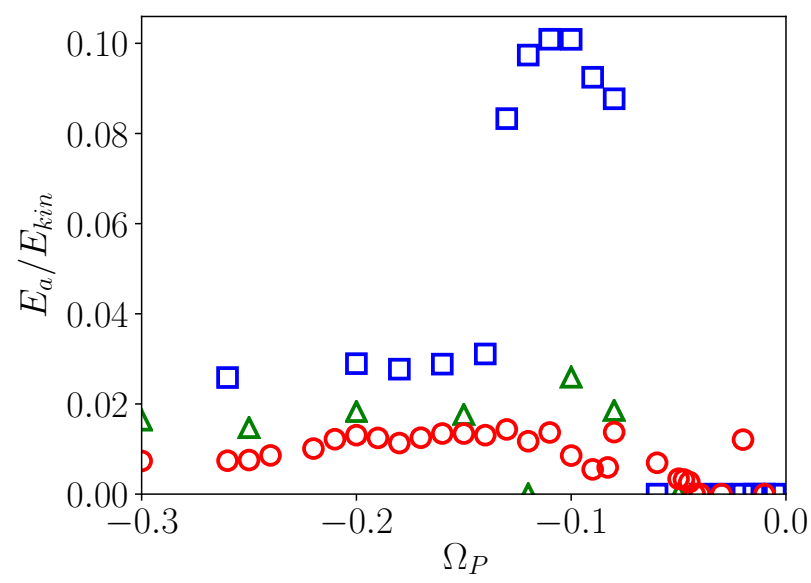

Figure 5.3: $\frac{E_{a}}{E_{k i n}}$ at $\mathrm{Ek}=2.5 \cdot 10^{-4}$ for $\eta=-0.7$ (squares), $\eta=-0.5$ (triangles), $\eta=$ 0.0 (circles)

with azimuthal wave numbers 2 and 1, similar to triad resonant modes in sec. 3.5. For $\Omega_{P} \leq-0.14 \frac{E_{a}}{E_{k i n}}$ stays roughly stable at 0.027 . The high maximum value of $\frac{E_{a}}{E_{k i n}}$ here is promising for dynamo action with reasonable amounts of energy put into the precessing fluid and therefore a possible, relatively low $\mathrm{Rm}_{\mathrm{c}, \mathrm{kin}}$ (sec. 4.2). This is of course relevant,
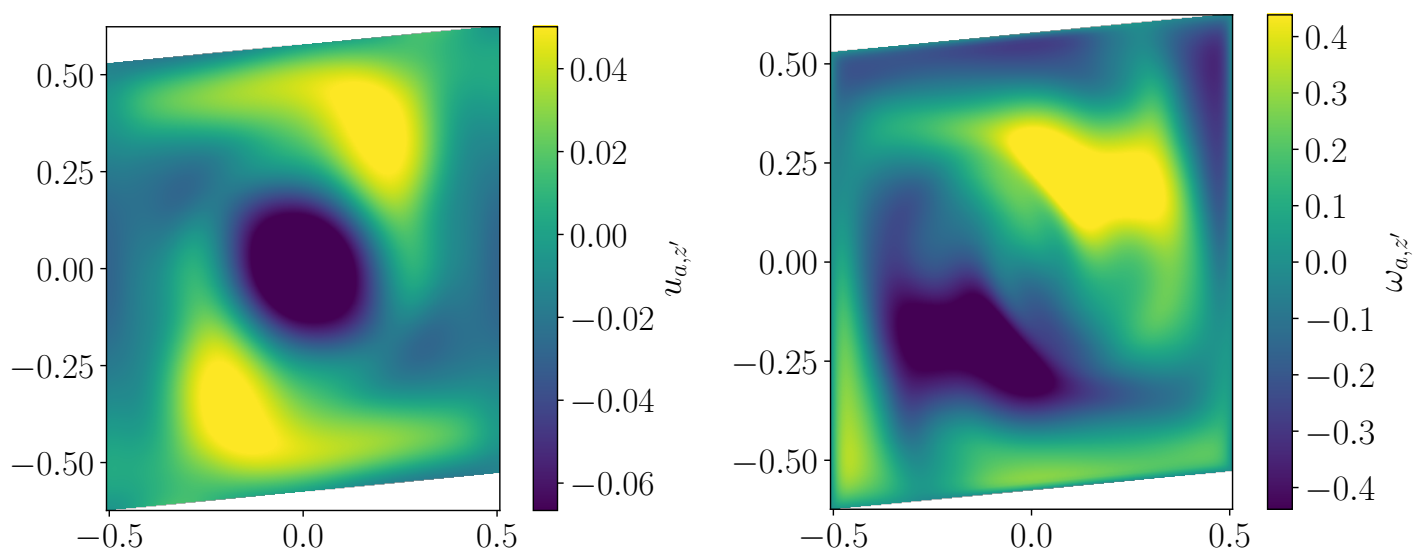

Figure 5.4: $u_{a, z^{\prime}}$ (left) and $\omega_{a, r^{\prime}}$ (right) for $\eta=-0.7$ at $\mathrm{Ek}=2.5 \cdot 10^{-4}$ and $\Omega_{P}=-0.1$ in the plane normal to $\boldsymbol{z}^{\prime}$ through the origin.

if $\frac{E_{a}}{E_{k i n}}$ is able to hold on this value even at low Ekman numbers. In fig. 5.5 this is done 


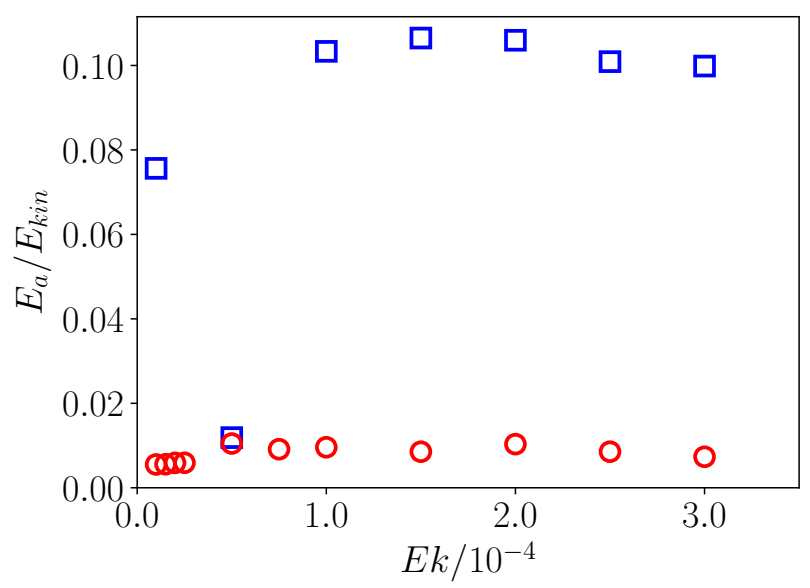

Figure 5.5: $\frac{E_{a}}{E_{k i n}}$ at $\Omega_{P}=-0.1$ for $\eta=-0.7$ (squares), $\eta=0.0$ (circles)

for $\Omega_{P}=-0.1$ down to $\mathrm{Ek}=1.0 \cdot 10^{-5}$. For $\mathrm{Ek} \geq 1.0 \cdot 10^{-4} \frac{E_{a}}{E_{k i n}}$ holds above 0.1, a multiple of the value for the cube. For $\mathrm{Ek}=5.0 \cdot 10^{-5}$ its value is comparable to the cube, caused by a strong increase in kinetic energy up to $E_{k i n}=0.054$ as in fig. 5.6 analogous to that seen in sec. 3.1.1. There, for high precession rates the flow gains a strong axisymmetric circulation (see fig. 3.48) and the threshold of the precession rate for this increase decreases with decreasing Ek. After passing this threshold, the fluid shows no sign of decreasing $E_{k i n}$ when further lowering Ek. This axisymmetric circulation breaks down at $\mathrm{Ek}=1.0 \cdot 10^{-5}$ by the cuboid with $\eta=-0.7$ in the contrary to the behavior by the cube, so that $E_{k i n}$ only amounts to 0.008 , and $\frac{E_{a}}{E_{k i n}}$ increases again although not to the value before.

\subsection{Kinematic Dynamo}

How far the extraordinary high amount of antisymmetric energy in relation to kinetic energy - and subsequently strong instabilities - affects the ability to drive a dynamo, can be examined by the same procedures established for the cube in sec. 4. Strictly speaking, a critical magnetic Prandtl number satisfying eq. 4.4 was sought by interpolating the growth factor $p$ between flows with differing Pm. This was used to calculate the critical full-flow magnetic Reynolds number $\mathrm{Rm}_{c, k i n}$ of eq. 4.6 and the rotational magnetic Reynolds number $\mathrm{Rm}_{c, \text { rot }}$ of eq. 4.7. Comparing $\mathrm{Rm}_{c, k i n}$ in cube and cuboid in fig. 5.7 for $\Omega_{P}=-0.1$, it is apparent, that the cuboid with $\eta=-0.7$ always undercuts 


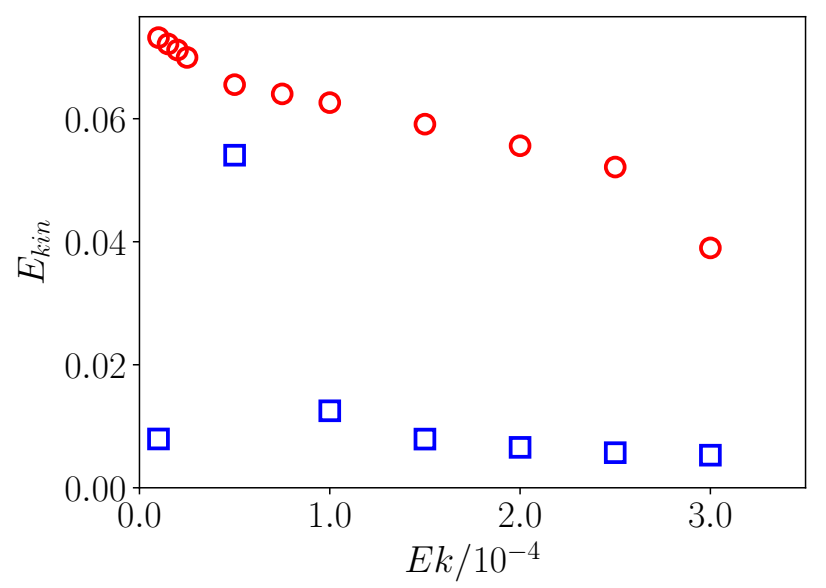

Figure 5.6: $E_{k i n}$ at $\Omega_{P}=-0.1$ for $\eta=-0.7$ (squares), $\eta=0.0$ (circles)

the performance of the cubic container at the same precession rate. While remaining on a level about $\mathrm{Rm}_{c, k i n} \approx 2000$ for all simulation runs with $\mathrm{Ek} \geq 1.0 \cdot 10^{-4}$, it varies more for any Ekman number below that. This of course is caused by the structural changes the flow experiences in this region as described in sec. 5.1. The discontinuation of the axisymmetric circulation at $\mathrm{Ek}=1.0 \cdot 10^{-5}$ does improve $\mathrm{Rm}_{c, k i n}$ again to 4284 , a third of what would be necessary for a cube. As explained in 4.2 this would also reduce the power hypothetical motors would need to achieve a functioning dynamo effect. Here, the difference of $\mathrm{Rm}_{c, k i n}$ between cube and chosen cuboid is strongly influenced by the value of $E_{k i n}$. To consider this without this influence, $\mathrm{Rm}_{c, r o t}$ can be used again. Since the chosen specific length scale is same for both compared containers $\left(L_{s}=1\right)$, this parameter directly correlates to the rotation velocity and henceforth the rotation frequency, a cuboid or cube needs to generate a magnetic field. Interestingly, fig. 5.8 presents $\mathrm{Rm}_{c, \text { rot }}$ for the cube and cuboid and shows little difference between the two. In contrast to $\mathrm{Rm}_{c, k i n}$ the critical value in the cuboid only varies by small amounts compared to the ones of the cube. This also means, that $\mathrm{Rm}_{c, \text { rot }}$ in the cuboid increases with decreasing Ek as it was already seen in fig. 4.8 for the cube and reduces the possibility to achieve a dynamo within the precession experiment of DresDYN (compare parameters given in sec. 4.3, tab. 4.1 and tab. 5.2). Of course, the cuboid also shows the same behavior in $L_{D}$ (defined in eq. 4.8) as the cube. So both geometries suffer from a strong increase in dissipation by decreasing Ek, hampering the ability to generate magnetic fields at low Ek. 


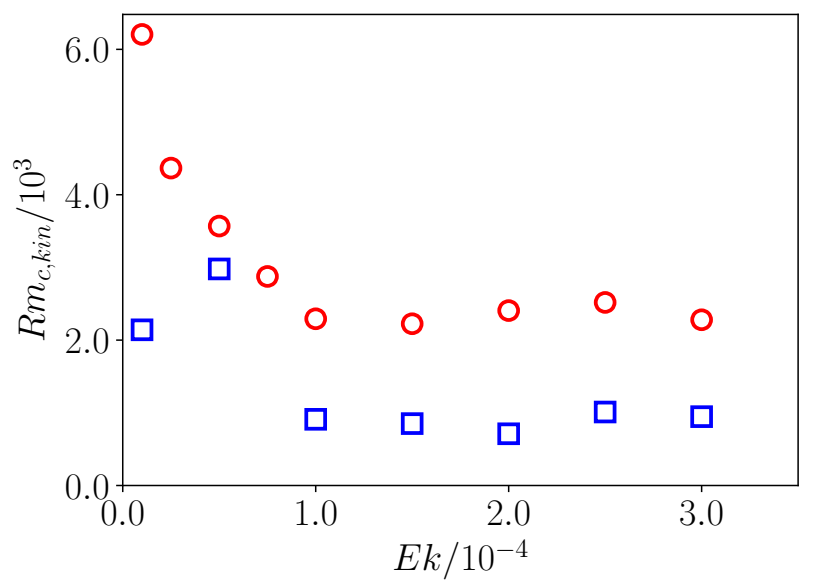

Figure 5.7: $\mathrm{Rm}_{c, k i n}$ at $\Omega_{P}=-0.1$ for $\eta=-0.7$ (squares), $\eta=0.0$ (circles)

\begin{tabular}{r|r|c|r|r|r|r|r|r}
\multirow{2}{*}{$\mathrm{Ek}$} & $E_{k i n}$ & $E_{a}$ & \multicolumn{2}{|c|}{$\mathrm{Pm}_{c}$} & \multicolumn{2}{|c|}{$\mathrm{Rm}_{c, k i n}$} & \multicolumn{2}{|c}{$\mathrm{Rm}_{c, r o t}$} \\
$\eta$ & -0.7 & -0.7 & -0.7 & 0.0 & -0.7 & 0.0 & -0.7 & 0.0 \\
\hline $2.5 \cdot 10^{-4}$ & $5.71 \cdot 10^{-3}$ & $5.76 \cdot 10^{-4}$ & 4.73 & 3.90 & 2022 & 5037 & 9464 & 7799 \\
$5.0 \cdot 10^{-5}$ & $5.40 \cdot 10^{-2}$ & $6.42 \cdot 10^{-4}$ & 0.91 & 0.99 & 5958 & 7138 & 9059 & 9858 \\
$1.0 \cdot 10^{-5}$ & $7.94 \cdot 10^{-3}$ & $6.00 \cdot 10^{-4}$ & 0.34 & 0.32 & 4284 & 12411 & 17001 & 16220
\end{tabular}

Table 5.2: Selected values of $\mathrm{Rm}_{c, k i n}$ and $\mathrm{Rm}_{c, \text { rot }}$ at $\Omega_{P}=-0.1$ for cuboid with $\eta=-0.7$ and the cube $(\eta=0.0)$ for comparison.

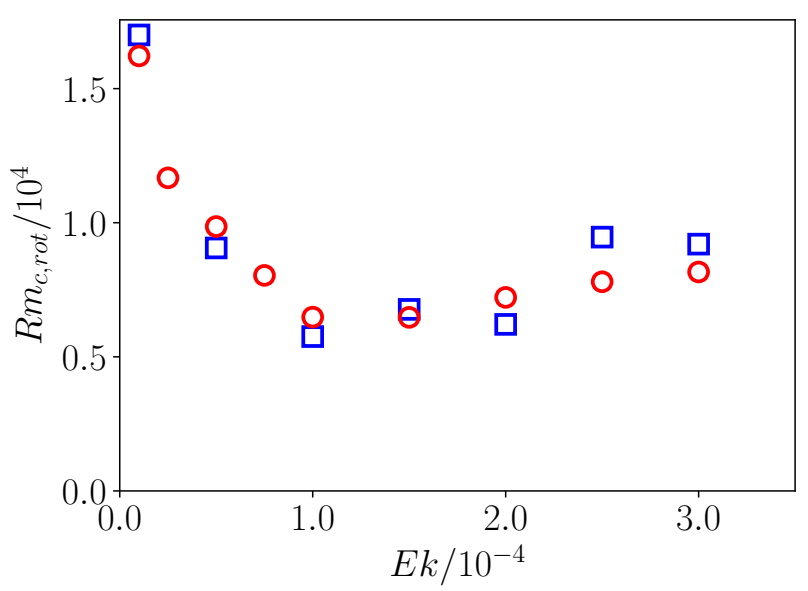

Figure 5.8: $\operatorname{Rm}_{c, \text { rot }}$ at $\Omega_{P}=-0.1$ for $\eta=-0.7$ (squares), $\eta=0.0$ (circles) 


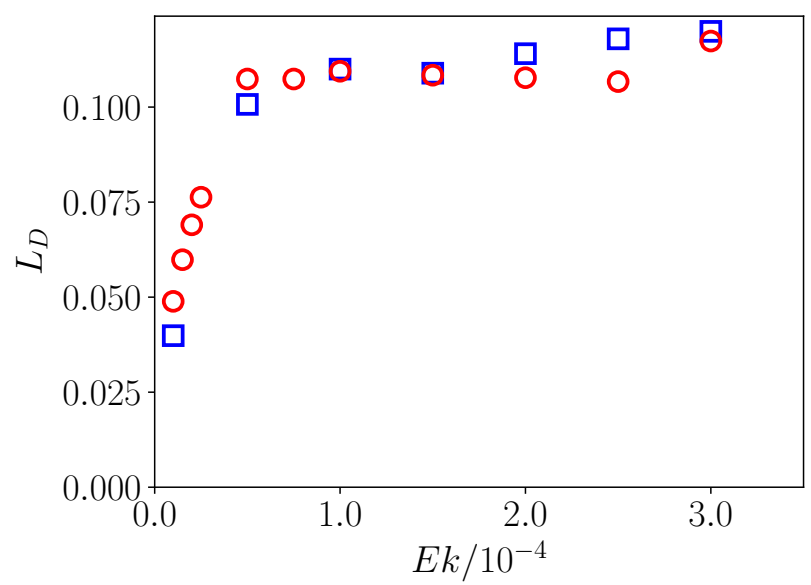

Figure 5.9: $L_{D}$ at $\Omega_{P}=-0.1$ for $\eta=-0.7$ (squares), $\eta=0.0$ (circles) 


\section{Herzenberg Dynamo}

With small modifications, a separating wall can be introduced into the simulation code, which divides the system into two fluid chambers. This may open the possibility for other mechanisms generating dynamos like the so-called Herzenberg dynamo[Herzenberg, 1958]. This concept will be further studied in the following section.

\subsection{Fundamentals}

The basic Herzenberg Dynamo as described in [Herzenberg, 1958] consists of two conducting spheres with radius $a$ and distance $2 d$ between them, rotating around their respective centres with rates $\boldsymbol{\omega}_{1}$ and $\boldsymbol{\omega}_{2}$ and axes not parallel to each other. They are immersed in a larger fluid filled sphere, with the same electromagnetic properties as the rotating spheres, itself surrounded by an insulator. With this setup, Herzenberg was able to predict possible dynamo action in this system, as long as $d \gg a$ and the condition of a finite radius of the larger sphere is fulfilled. Further work was done by others to examine the possibility of an infinite conducting sphere or multiple rotors[Gibson, 1968a, Gibson, 1968b] and inspired experiments by [Lowes and Wilkinson, 1963, Lowes and Wilkinson, 1968], who could see actual dynamo action, by tilting two cylinders each embedded in a block of the same material, a conducting iron alloy. Possible applications in astrophysical context were discussed by [Dolginov and Urpin, 1979], studying the dynamo as an effect in binary star systems. [Brandenburg et al., 1998] started numeric simulations and found not only strong dynamo action but also an area of oscillatory magnetic field in systems. For this, they modified the Herzenberg setup by placing two rotors into a periodical box with very large extent in respect to the distance of the rotors, again consisting completely of materials of the same conductivity. When the centers of the rotors with radius $a$ were at points $\boldsymbol{r}_{\mathbf{1}}=(0,0, d)$ and $\boldsymbol{r}_{\mathbf{2}}=(0,0,-d)$ and the rotation axes tilted by $\boldsymbol{\omega}_{1}=\left(-\frac{1}{2} \sin (\phi), \frac{1}{2} \cos (\phi), 0\right)$ and $\boldsymbol{\omega}_{2}=\left(\frac{1}{2} \sin (\phi), \frac{1}{2} \cos (\phi), 0\right)$ (like pictured in fig. 6.1), there was a definitive positive growth rate for $|\phi|>90^{\circ}$, while an oscillating solution 
was found for $0^{\circ}<|\phi|<90^{\circ}$, confirming a prediction by Moffatt[Moffatt, 1978] which foresaw that

$$
\mathrm{Rm}^{-2} \propto \sin ^{2} \phi \cos \phi .
$$

Also they were able to expand Herzenberg's model to systems, where $d \gg a$ was not necessary anymore.

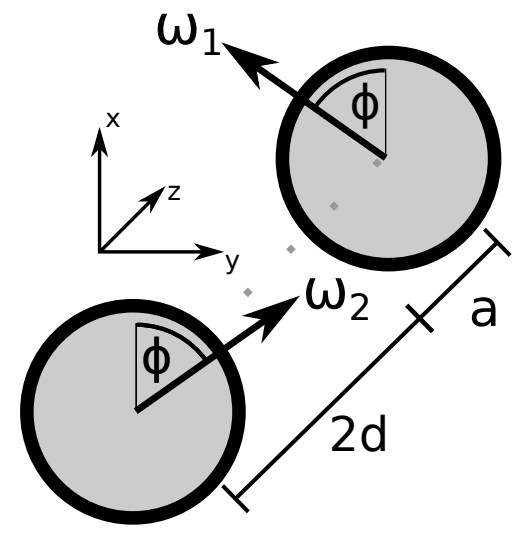

Figure 6.1: The model as it was used by [Brandenburg et al., 1998]: Two spheres of radius $a$ and at a distance $2 d$ of each other rotate around axes, which are tilted inside the $x$ - $y$-planes by an angle $2 \phi$ against each other. The electromagnetic properties of the spheres and their surrounding are the same. The confining bounding box necessary for a simulation is not visualized.

Now, using the simulation code proposed in section 2.2 , an analogous two-rotor system is built by introducing immersed boundaries into cuboids of length $L_{z}$ in $z$-direction. These inner boundaries create a solid barrier normal to the z-axis with a thickness $L_{s}$ as in figure. 6.2 and divide the cuboid into two smaller cuboids $C_{1}$ and $C_{2}$ of length $L_{1}$ and $L_{2}$ so that

$$
L_{z}=L_{1}+L_{s}+L_{2} .
$$

Since the boundary conditions at the outer boundaries in the simulations in this work are designed as free-slip (see 2.2.1), this is the choice for the inner boundaries facing towards the cuboids $C_{1}$ and $C_{2}$. The magnetic field on the other hand will be able to propagate through the barrier unhindered. The space between $C_{1}$ and $C_{2}$ will be seen as solid so that

$$
\boldsymbol{u}_{\text {mantle }}\left(L_{1}<z<L_{1}+L_{s}\right)=0 .
$$

From this point on, the setups at different $L_{1}, L_{S}, L_{2}$ will be explicitly identified by the form $\left(L_{1}, L_{S}, L_{2}\right)_{H}$. $L_{x}$ and $L_{y}$ is always set to 1.0. As before, the whole container 
rotates at fixed $\omega_{D}$, but the fluid inside $C_{1}$ and $C_{2}$ rotates around fluid axes specific two the containers' forms. This system is predestined to run as a kinematic dynamo as before. In consequence, the flows inside the different chambers will work as rotors undeterred by the influence of the magnetic field.

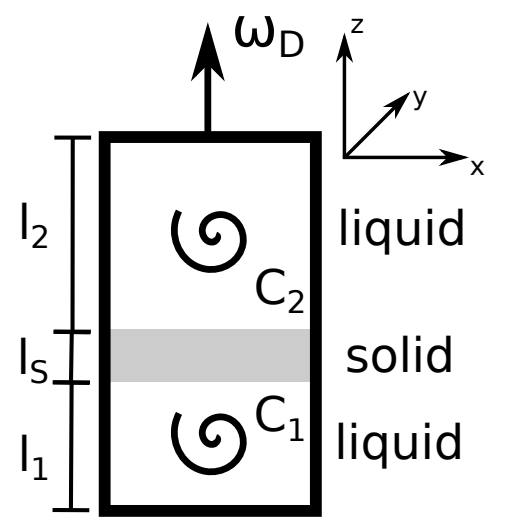

Figure 6.2: The original cuboid model is modified by implementing a solid phase which divides the inner space into two smaller cuboids. The solid phase shows the same electromagnetic properties like the remaining fluid phases, but got itself zero velocity in the mantle system. The boundaries facing the fluid phases use the same free-slip conditions like the outer boundaries.

\subsection{Results}

First runs with inner boundaries test the functionality of this newly introduced feature. $C_{1}$ takes the measures of a cube $\left(L_{x}, L_{y}, L_{1}\right)=(1.0,1.0,1.0)$, while the solid barrier thickness is $L_{s}=1.1$. For the tests, $L_{2}=0$, so that $C_{2}$ vanishes as depicted in fig. 6.3. Hence, the notation is $(1.0,1.1,0.0)_{H}$. Parameters are set to $\mathrm{Ek}=10^{-4}, \alpha=60^{\circ}$, $\Omega_{P}=-0.1$ and $\mathrm{Pm}=2$, but are only used for demonstration purposes. The velocity in this system equals, like designed, zero for the whole solid phase, an example is seen in fig. 6.4 along a line at $(x, y)=(-0.25,-0.25)$ : for $0 \leq z<1.1$ all velocity components are constant, but vary for $1.1 \leq z \leq 2.1$. Since the inner boundary is stress-free like the outer boundaries, the components $u_{x}$ and $u_{y}$ can take any value at $z=1.1$ and can be discontinuous here (and very likely are), while the $u_{z}$-component is bound to be zero caused by the no-flux condition (see sec. 2.2.1). Of course, the dynamo effect can only generate a magnetic field inside the fluid phase, and so it looks like pictured in fig. 6.5 at the same parameters as fig. 6.4. While the fluid phase does include non-trivial 


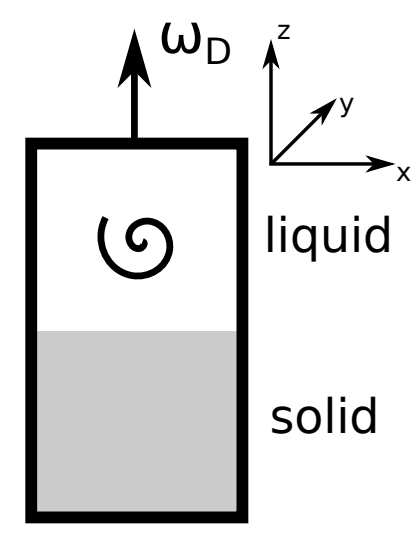

Figure 6.3: For first test runs the solid barrier is extended to the lower boundary of the whole container, so that cuboid $C_{2}$ vanishes and the field in $C_{2}$ only interacts with the solid phase.

values for all magnetic components, they vanish very fast into the solid phase. At the transition, on the other hand, a small peak exists, which is more detailed in 6.6: $B_{x}$ and $B_{y}$ both show peaks at the transition, while $B_{z}$ has no equivalent behavior, analogous to the discontinuous curves of $u_{x}$ and $u_{y}$. The magnetic energy $\hat{\epsilon}_{B}$ along the rotation axis defined as

$$
\hat{\epsilon}_{B}(z)=\frac{\iint \boldsymbol{B}(x, y, z)^{2} \mathrm{~d} x \mathrm{~d} y}{\iint \mathrm{d} x \mathrm{~d} y}
$$

and displayed in 6.7 shows exponential decay inside the solid phase, which ends in a weak mode, with a maximum several orders of magnitude below the energy inside the fluid phase. Already after a distance of $\Delta z=0.1$ after the transition, the energy dropped three orders of magnitude. Also present is the peak at the border, probably generated by the discontinuous transition. Now, allowing a second fluid phase again with $C_{2}$, at first, models with different sizes of $C_{1}$ and $C_{2}$ were done, but further on with $L_{S}=0.1$ and $L_{z}=2.1$ and $L_{x}=L_{y}=1 . \quad L_{S}$ is set to 0.1 to emulate only a small barrier between the two containers, which would be reasonable in a hypothetical experiment. For the first model, the length ratio of the phases was set to $(1,0.1,1)_{H}$ to create a simple system with two identical rotors with parallel rotation axes, as it can be seen on the left side of fig. 6.8 and 6.9. The precession rates $\Omega_{P}=-0.04$ and $\Omega_{P}=-0.35$ were chosen, because a system of a simple cube $\left(L_{z}=1\right)$ like in 3.1 was stable at both of these points and no instabilities should influence the flow. Nevertheless cubes at these points get a relatively low critical magnetic Prandtl number $\mathrm{Pm}_{c}<10$ as seen in the last column in tab. 6.1, reducing the capacity to see effects only introduced by the setup. Looking at the magnetic field at $\Omega_{P}=-0.04$ (fig. 6.8,right side) one can see the 


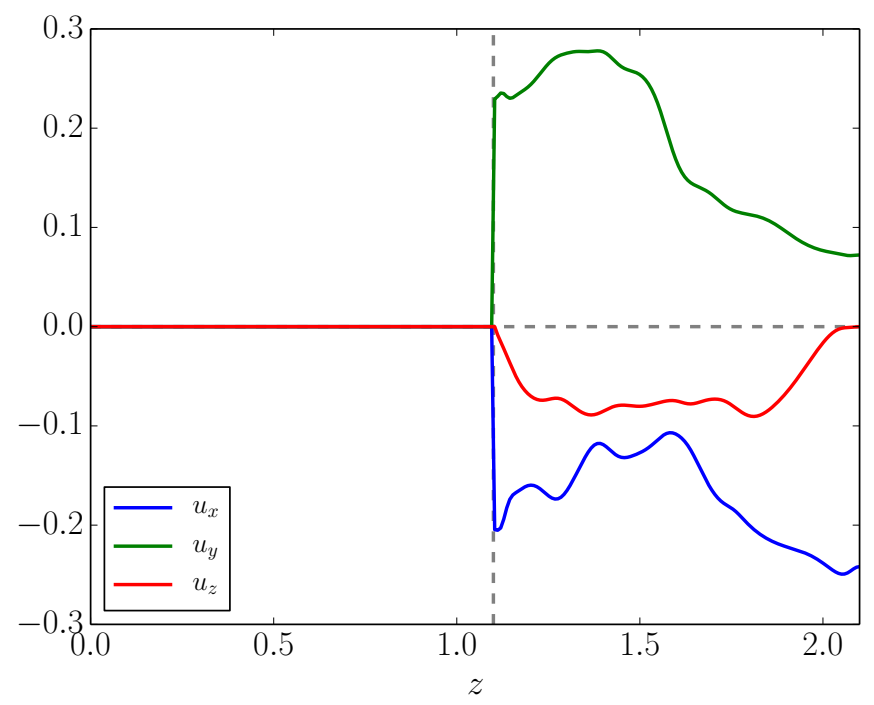

Figure 6.4: The velocity profile at $\mathrm{Ek}=10^{-4} ; \Omega_{P}=-0.1 ; \mathrm{Pm}=2$ in a container of form $\left(L_{x}, L_{y}, L_{z}\right)=(1,1,2.1)$ but with $L_{s}=1.1, L_{1}=1$ and $L_{2}=0$ shows the transition between solid and fluid phase at $(x, y)=(-0.25,-0.25)$. The no-flux boundary condition in $z$-direction provide for a continuous curve in $u_{z}$ at the transition $z=1.1$ (dashed vertical line), but $u_{x}$ and $u_{y}$ become discontinuous because of the stress-free condition.

propagation of it through the solid barrier, which decays fast, but also largely maintains the symmetry inside each container for the most part. The magnetic energy $\hat{\epsilon}_{B}(z)$ of eq. (6.4) inside this system (see fig. 6.10) also decays as in the fluid-solid-model in 6.3 but has a minimum in the middle of the solid phase, since the same magnetic field propagates from the second container from the other side. The same behavior shows $\hat{\epsilon}_{B}(z)$ for $\Omega_{P}=-0.35$ in fig. 6.11 , although magnetic energy is much more intense at the outer borders with pseudo vacuum boundary conditions while the permeability of the inner boundaries allows for the magnetic energy to disperse into the solid boundary. The resulting critical magnetic Prandtl number $\mathrm{Pm}_{c}$ in tab. 6.1 (first column) show for $\Omega_{P}=-0.35$ no significant difference to the one obtained from a cube, for $\Omega_{P}=-0.04$ $\mathrm{Pm}_{c}$ was reduced by roughly a third from $\mathrm{Pm}_{c}=9.07$ in a simple cube to $\mathrm{Pm}_{c}=5.24$ in the Herzenberg setup. Since the rotation axes in the symmetrical setup should be identical, the Herzenberg Dynamo is not in effect as long as the axes form an angle of $\phi=0$. The same setup at different parameters $\mathrm{Ek}=1.0 \cdot 10^{-4} ; \Omega_{P}=-0.1$ does not show any similar effect, but got $\mathrm{Pm}_{c}=1.5$, which is not as good as the critical Prandtl number in the respective simple cube, $\mathrm{Pm}_{c}=1.3 . \quad$ A better way to generate a Herzenberg 


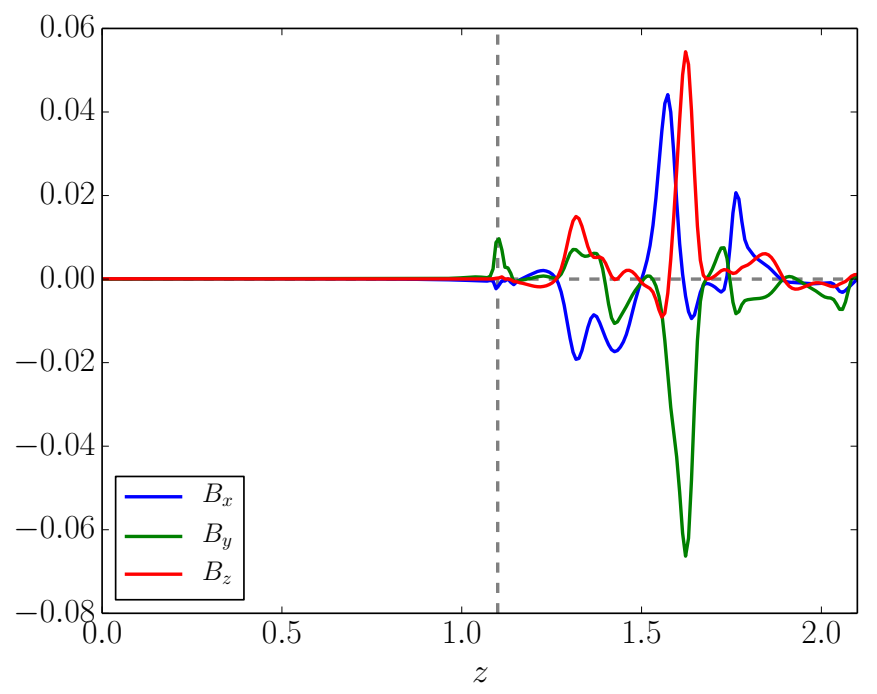

Figure 6.5: The magnetic components for the same parameters as in fig. 6.4 vanishes in the solid phase except for a small peak at $z=1.1$.

\begin{tabular}{l|c|c|c|c|c|c}
$\Omega_{P}$ & $(1,0.1,1)_{H}$ & $(0.66,0.1,1.34)_{H}$ & $(0.5,0.1,1.5)_{H}$ & $L_{z}=0.5$ & $L_{z}=1.5$ & $L_{z}=1.0$ \\
\hline-0.35 & $6.25 \pm 0.25$ & $9.69 \pm 0.31$ & $3.87 \pm 0.13$ & $3.54 \pm 0.46$ & $4.81 \pm 0.19$ & $6.55 \pm 0.55$ \\
-0.04 & $5.24 \pm 0.24$ & $>30$ & $>30$ & - & - & $9.07 \pm 0.07$
\end{tabular}

Table 6.1: Resulting critical magnetic Prandtl numbers $\mathrm{Pm}_{c}$ for a range of different containers and two different precession rates $\Omega_{P}=-0.04$ and $\Omega_{P}=-0.35$, but all at $\mathrm{Ek}=5 \cdot 10^{-4}$. The first three container used a solid barrier to simulate Herzenberg dynamos while the last three were simple cuboids with accordingly chosen $L_{z}$ for comparison purpose.

Dynamo in this setup should be a system of different formed rotors. Therefore the same extents of the total container were chosen, $\left(L_{x}, L_{y}, L_{z}\right)=(1,1,2.1)$, but the solid barrier was located at other $z$-coordinates, so that $\left(L_{1}, L_{s}, L_{2}\right)_{H}=(0.66,0.1,1.34)_{H}$ and $\left(L_{1}, L_{s}, L_{2}\right)_{H}=(0.5,0.1,1.5)_{H}$. The different form of the containers also implies different flows inside, which on itself could carry a dynamo. As seen in tab 6.1 even simple cuboid container of $L_{z}=0.5$ and $L_{z}=1.5$ get critical magnetic Prandtl numbers around $\mathrm{Pm}_{c}=3.5$ and $\mathrm{Pm}_{c}=4.8$ at $\Omega_{P}=-0.35$. The equivalent Herzenberg setup $(0.5,0.1,1.5)_{H}$ does not deviate from this results with a critical number $\mathrm{Pm}_{c}=3.9$ and it seems to be more caused by the dynamo effects solely generated in the container $C_{2}$ with $L_{2}=1.5$. Looking into the normalized magnetic energy of this system in fig. 6.12, 


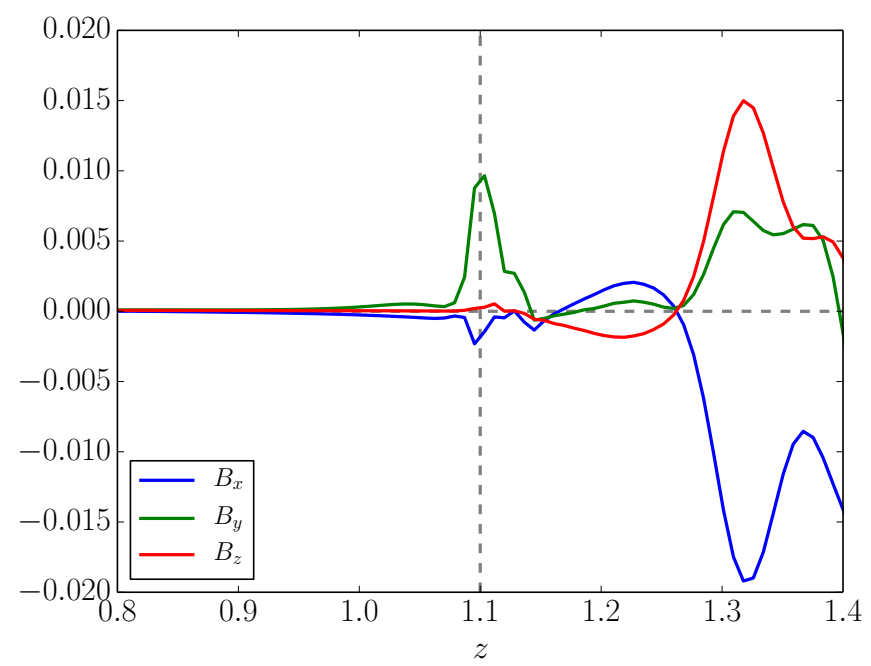

Figure 6.6: The peak in magnetic components at $z=1.1$ shown in a smaller cut-out of fig. 6.5 is more specifically in components $B_{x}$ and $B_{y}$ while in $B_{z}$ no peak is seen.

it is apparent, that it is actually concentrated in the container $C_{2}$ while it decays in the solid phase and only a residual remains in $C_{1}$. For the $(0.66,0.1,1.34)_{H^{-}}$-setup a similar behavior can be seen, although here the container $C_{1}$ builds up the main share in $\hat{\epsilon}_{B}(z)$. For $\Omega_{P}=-0.04$ the critical magnetic Prandtl number for the last two cases even must be over 30, which is a strong indicator, that no dynamo action takes place, not even oscillatory behavior as it could be assumed for small angle between rotation axes by analysis[Brandenburg et al., 1998].

Until now, all simulation cases were done with easily chosen setups, but since the original Herzenberg setup demands large angles between rotation axes, one can try and use known and understood flows to start with systems, which are known to deviate strongly in their position of fluid axis. Two well studied systems in this work are the cube in chapter 3 and a cuboid with ellipticity $\eta=-0.7$ (amounting to $L_{z}=1.8257$ ) in chapter 5 . The two defining angles $\theta_{F}$ and $\varphi_{F}$, respectively the axial and azimuthal angle of the fluid axis $\boldsymbol{\omega}_{\boldsymbol{F}}$ as defined in equation 3.2, were used to identify such systems of interest by studying these values at $\mathrm{Ek}=2.5 \cdot 10^{-4}, \alpha=60^{\circ}$ and different $\Omega_{P}$. For the axial angle $\theta_{F}$, fig. 6.13 shows the difference in an area with $\Omega_{P}<-0.08$, while for $\Omega_{P}>-0.08$ the values are roughly the same. For the azimuthal angle $\varphi_{F}$ the differences for $\Omega_{P}>-0.08$ diverge more and constitute to values like $\Delta \varphi_{F}=35^{\circ}$ at $\Omega_{P}=-0.1$ and even $\Delta \varphi_{F}=99^{\circ}$ at $\Omega_{P}=-0.18$. As described in 4 the critical magnetic Prandtl number for a cube at 


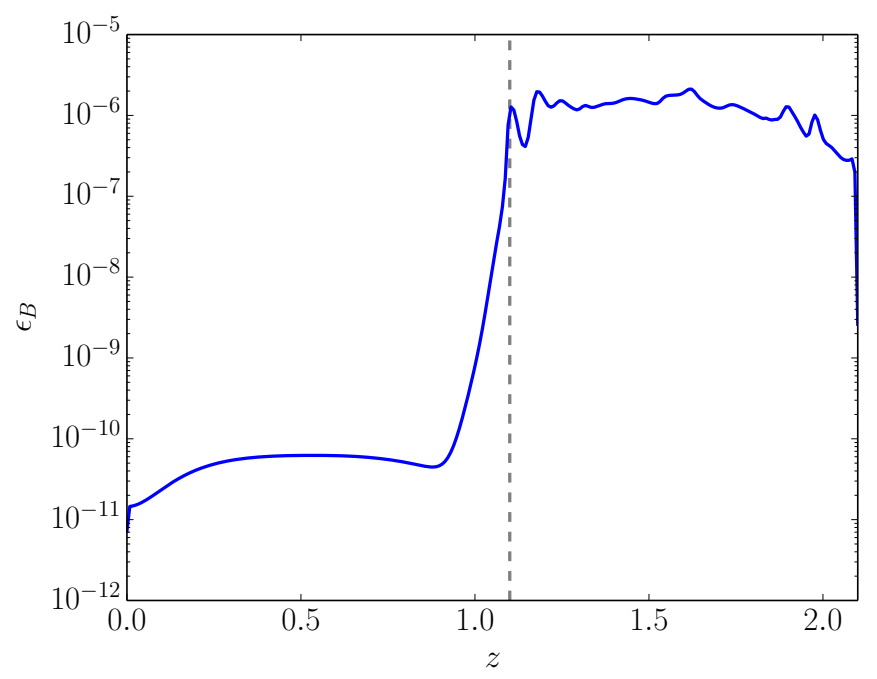

Figure 6.7: The normalized magnetic energy decays exponentially in the solid phase and develops a very weak mode. At the $z=1$.1-border a small peak exists, likely generated by the discrete transition between the two states.

$\Omega_{P}=-0.18$ is relatively low $\left(\mathrm{Pm}_{c}=1.96\right)$, while the cuboid with $L_{z}=1.8257$ has one of double that value at $\mathrm{Pm}_{c}=3.9$. The adequate Herzenberg setup consisting of those two containers does not manage to decrease below these values neither in the $\Omega_{P}=-0.18$ case nor at $\Omega_{P}=-0.1$ as can be seen in tab. 6.2.

\begin{tabular}{l|c|c|c}
$\Omega_{P}$ & $L_{z}=1$ & $L_{z}=1.8357$ & $(1,0.1,1.8257)_{H}$ \\
\hline-0.1 & 3.9 & 4.7 & 4.4 \\
-0.18 & 1.96 & 3.6 & 3.9
\end{tabular}

Table 6.2: The critical magnetic Prandtl numbers $\mathrm{Pm}_{c}$ for the $(1,0.1,1.8257)_{H^{-}}$-setup and the adequate stand-alone containers are all at the same level between 3.9 and 5 with the exception of the very low $\mathrm{Pm}_{c}=1.96$ for a cube at $\Omega_{P}=-0.18$. The Herzenberg setup never manages to undercut the critical numbers of the sole containers. 

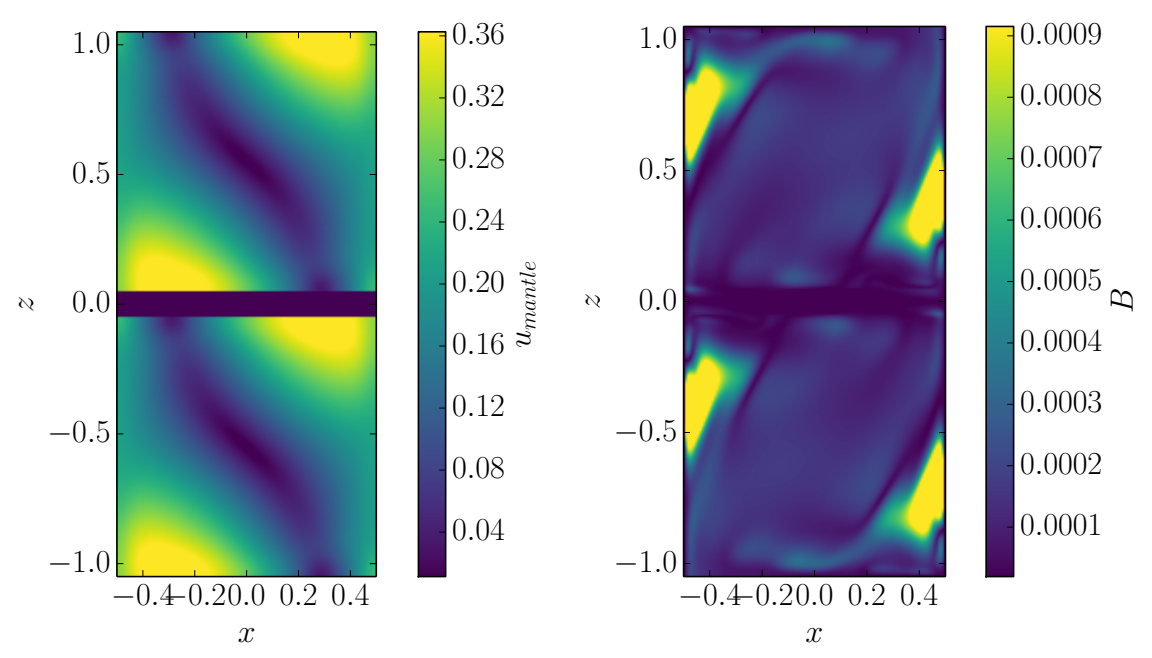

Figure 6.8: The Herzenberg setup at $\mathrm{Ek}=5 \cdot 10^{-4}, \Omega_{P}=-0.04$ generates two identical flows in the mantle velocity $\boldsymbol{u}_{\text {mantle }}$ (left side) separated by the notable solid barrier between them as seen here in a cut through the center of the total container alongside the $z$ - $x$-plane. The magnetic field $\boldsymbol{B}$ (right side) at $\mathrm{Pm}=$ 6 , likely produced by shearing effects on the sides, can be seen propagating into the solid phase from both sides.
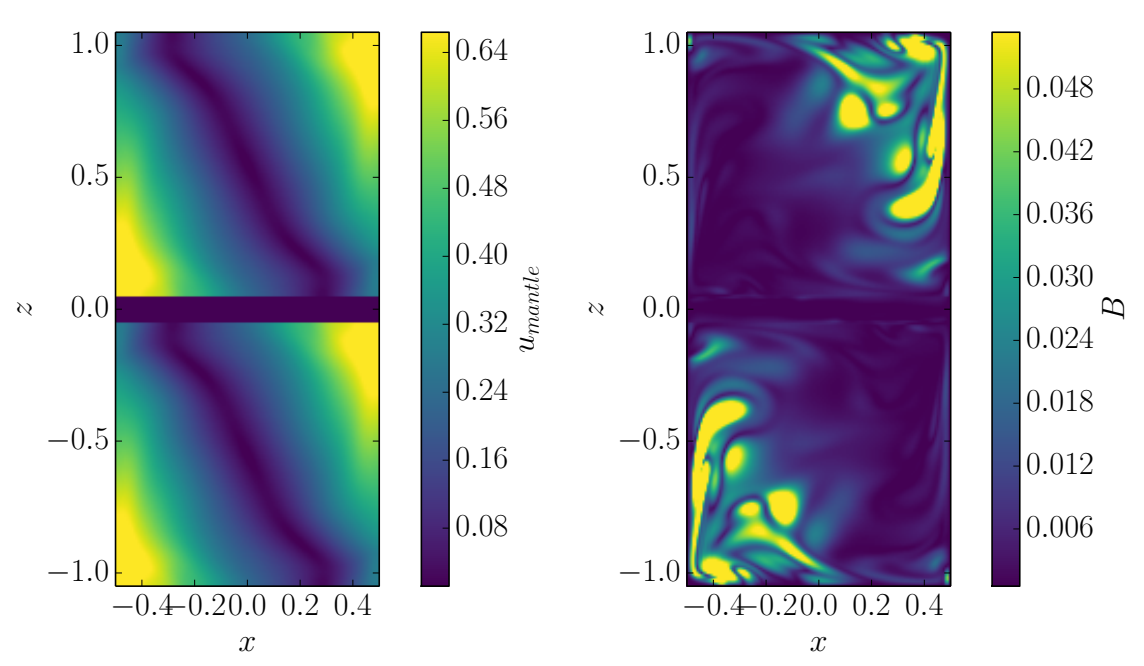

Figure 6.9: Similar to fig. 6.8 the mantle velocity $\boldsymbol{u}_{\text {mantle }}$ (left side) at $\mathrm{Ek}=5 \cdot 10^{-4}$, $\Omega_{P}=-0.35$ is identical in both containers, as is the magnetic field $\boldsymbol{B}$ (right side), here at $\mathrm{Pm}=7$, even though it seems to be more complicated. 


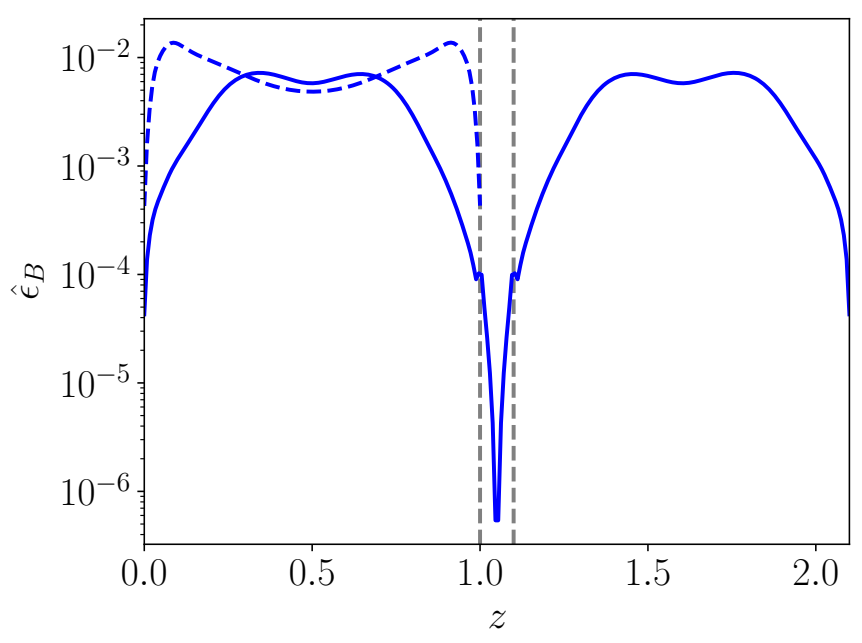

Figure 6.10: The normalized magnetic energy $\hat{\epsilon}_{B}(z)$ for the $(1,0.1,1)$-setup (continuous line) at $\Omega_{P}=-0.04, \mathrm{Ek}=5 \cdot 10^{-4}$ is at maximum amidst the containers while it distributes more homogeneous along the $z$-axis in a cube (dashed line) at same parameters. At the solid barrier, the Herzenberg setup shows the peak in the energy typical for the boundary transition.

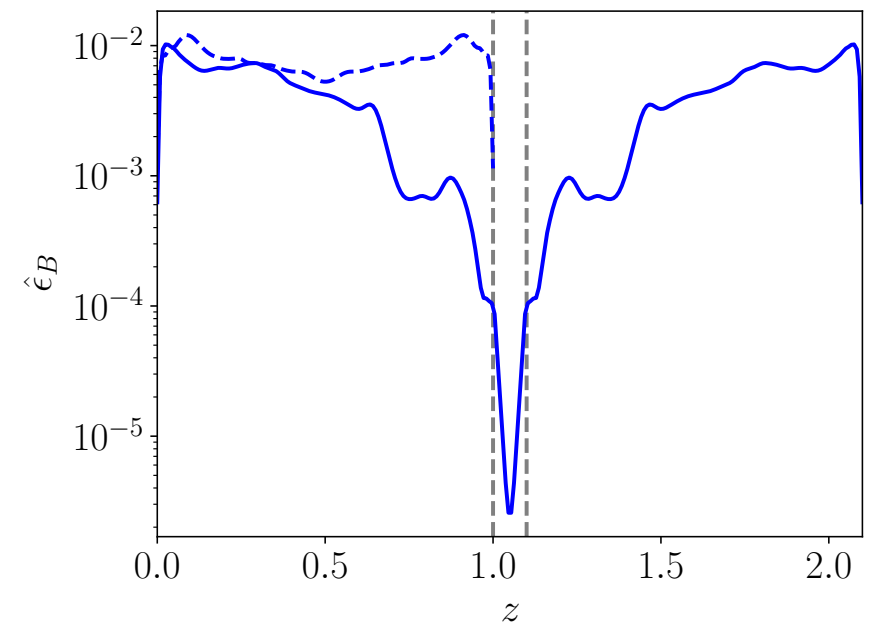

Figure 6.11: The normalized magnetic energy $\hat{\epsilon}_{B}(z)$ for the $(1,0.1,1)_{H}$-setup (continuous line) at $\Omega_{P}=-0.35, \mathrm{Ek}=5 \cdot 10^{-4}$ decreases from the outer borders of $z$ to the middle of the whole container. The matching cube (dashed line) has a much more even distribution. 


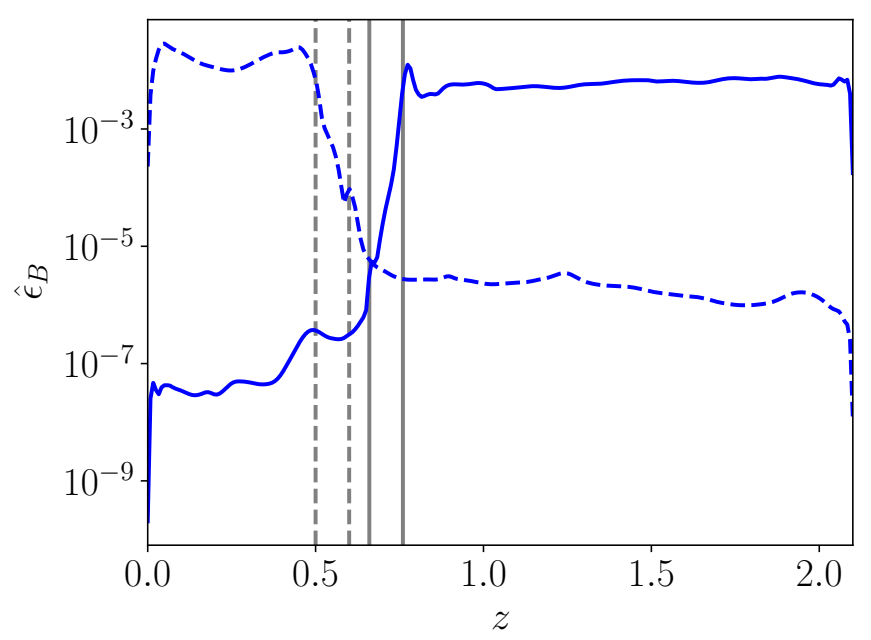

Figure 6.12: The normalized magnetic energy $\hat{\epsilon}_{B}(z)$ for the $(0.5,0.1,1.5)_{H}$-setup (continuous line) and the $(0.66,0.1,1.34)_{H}$-setup (dashed line) shows the concentration of the magnetic energy inside one of the containers which is most likely produced by the flow inside the corresponding container alone, while in the opposite container only a small remaining magnetic field exists.

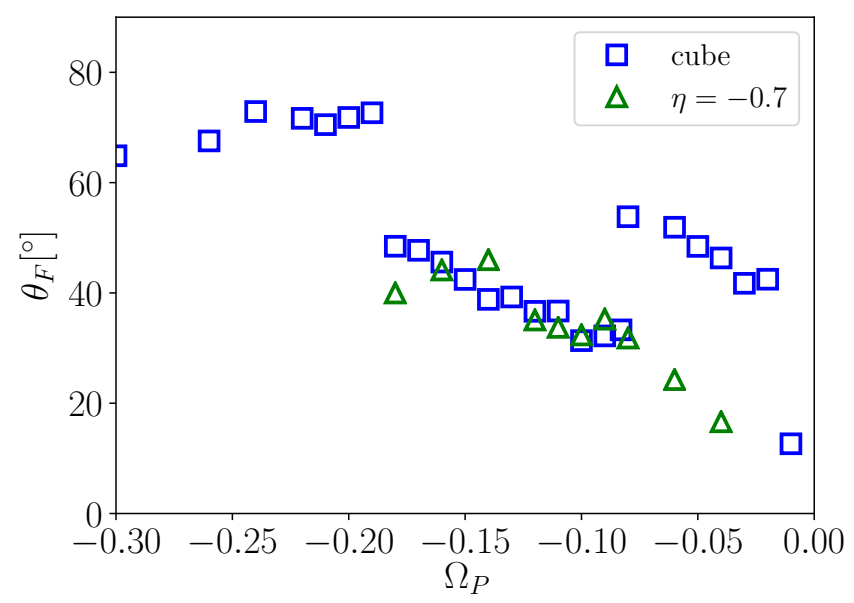

Figure 6.13: The axial angle $\theta_{F}$ of the fluid axis does not differ greatly between cube and cuboid with $\eta=-0.7$ for $\Omega_{P}<-0.08$. 


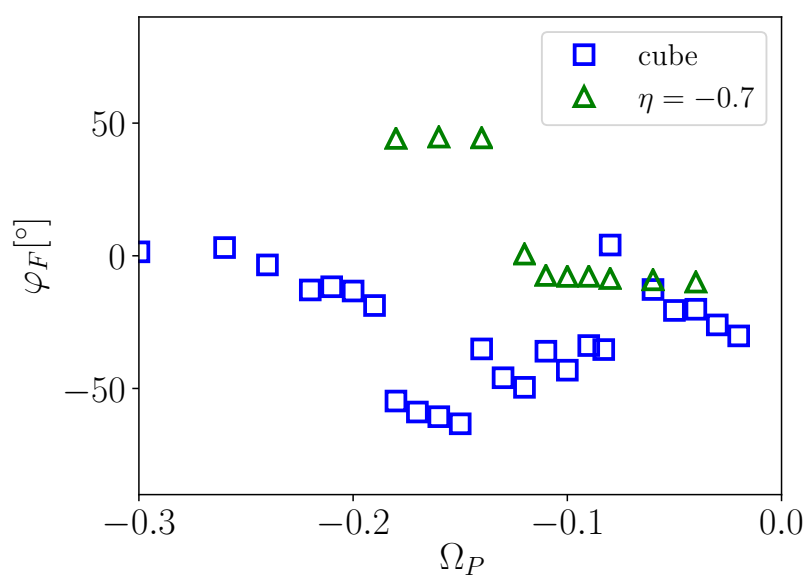

Figure 6.14: The azimuthal angle $\varphi_{F}$ for cube and cuboid with $\eta=-0.7$ get two distinct nontrivial values, in the regions $\Omega_{P} \in[-0.18:-0.14]$ and $\Omega_{P} \in$ $[-0.13,-0.09]$. 


\section{Conclusion}

\subsection{Hydrodynamics}

The examination of the flow in a cubic container revealed many different hydrodynamic effects. The correct interpretation of these effects in relation to other known geometries is of importance and will be discussed in this section.

\subsubsection{Single Vortex State}

One of the most notable features of the flow was the single vortex state at low Ekman numbers described in sec 3.3. It was shown, that a cyclonic vortex forms in the center of the container, rotating faster than the container itself. With the radius of this superrotational vortex $R_{\text {vortex }}$ as unit of measurement this work was able to identify a phase before the transition to turbulence for a broad range of Ekman numbers down to $\mathrm{Ek}=1.0 \cdot 10^{-5}$. But this kind of state of behavior is not unheard of in precessing systems: [Mouhali et al., 2012] observed in a cylindrical container at $\mathrm{Ek} \approx 1.8 \cdot 10^{-6} \mathrm{a}$ phase rich with cyclonic vortices which in some cases unified to a central single vortex but did not further classify this specific effect. The description of a number of cyclones collapsing into one mirrors effects later seen by [Lin et al., 2015]: Here, in a precessing sphere at $\mathrm{Ek} \approx 7.5 \cdot 10^{-6}$, small scale cyclones were found to merge into large vortices. However, instead of one central vortex, few vortices rotating around the center axis are maintained and, similar to the vortex in this work, disappear and reemerge for some time. This is very likely the effect of an inverse cascade, transporting energy from small scale structures to large scales. For this work such a connection was not as conclusive, since no distinctive phase with small scale cyclones was observed which could form into the single central vortex. Partly, this may be caused by the chosen unit of measurement of $R_{\text {vortex }}$, which exclude any vortices that do not fulfill the condition of positive vorticity and is restricted to the two-dimensional meridional plan, while possible effects may be 
bound to the edges of the container, requiring a three-dimensional procedure to investigate. One of the main restrictions on examining vorticity is the inability to distinguish whether it is caused by shearing or by an actual cyclonic vortex. This can be mitigated by the classification used by [Chong et al., 1990], defining vortices by the imaginary part of eigenvalues of the rate-of-strain tensor, and applied by [Vorobieff and Ecke, 1998], [Vorobieff and Ecke, 2002]. The investigation of this particular unit of measurement will further shed light on the complexion of the single vortex phenomenon. In comparison to the other studied hydrodynamics effects on the flow, the single state vortex is the most promising one, since its strength does increase for lower Ekman number over the whole investigated range. The axisymmetric modes as well as the triadic resonance interactions vanish or at least decrease for low Ekman numbers hinting at negligible influence for flows at $\mathrm{Ek}<1.0 \cdot 10^{-5}$. The single vortex state becomes excited at smaller precession rate for smaller Ekman numbers and is therefore more important for systems with weak precession.

\subsubsection{Axisymmetric modes}

The importance of axisymmetric modes for dynamo action is at least known since [Dudley, 1989] proved different axisymmetric rolls in a spherical container as formidable dynamos. With the work of [Giesecke et al., 2018] for DRESDyn, it became clear, that these form of flows is also relevant for precessing systems, as modes in shape of axisymmetric double roles become noticeable in experiments with cylinders. They occur for a small range of precession rates and, interestingly, by increasing the Reynolds number - which is anti-proportional to the Ekman number - the range becomes smaller and the modes stronger respective to the spin-over mode. The exact cause for their excitation is not yet known, but interaction between other inertial waves is possible [Meunier et al., 2008]. In the water experiment no dominant time-dependent behavior was noticed at any Ekman number and the flow with axisymmetric modes was laminar, even at Ek $<1.0 \cdot 10^{-5}$. In the cube, however, lowering the Ekman number causes the axisymmetric modes to first oscillate, starting at $\mathrm{Ek}=3.0 \cdot 10^{-4}$ and finally breakdown into chaotic regimes as described in section 3.4 decreasing the mean value of $E_{m=0, z} / E_{m \neq 0, z}$. This behavior seems unique and the precise relations between it and the use of a cube as rotating container are not clear, but simulation runs with no-slip boundaries show no oscillating behavior or tendencies to breakdown. This may be induced by the different level of axisymmetric azimuthal motion: The free-slip solutions feature a strong 
geostrophic mode simultaneously to the axisymmetric meridional motion, while the noslip boundary conditions obviously impede it by reducing the possible velocity alongside the boundaries. This strong azimuthal motion can generate instabilities by i.e. violating the Rayleigh stability criterion or possibly generating shear effects in the corners. This is very likely not the explanation here, since azimuthal motion between stable and breakdown cases for simulations with free-slip boundary condition show no correlation and are in fact relatively similar in magnitude. The oscillations of $E_{m=0, z} / E_{m \neq 0, z}$ occur simultaneously to $R_{\text {vortex }}$ of single vortex state also oscillating with the same frequency as seen in fig. 3.45. However, the phase difference - the maxima of $R_{\text {vortex }}$ arise when $E_{m=0, z} / E_{m \neq 0, z}$ decreases - and the disappearance of axisymmetric modes at regions with strong single vortex state and a low Ekman number implies that the single vortex state impedes axisymmetric modes in a yet unknown way. With new approaches for insight into the modal structure in cubic geometries the exact form of energy transfer into axisymmetric modes could be easily investigated and therefore a better understanding be reached in which way the energy transfers into axisymmetric modes and how the vortex state could hinder this. However, even the existence of this form of axisymmetric form gives away interesting information on the role of axisymmetric modes in a precessing system. [Giesecke et al., 2018] argues, that the main causes may be interactions with the no-slip conditions or the formation of internal shear layer. The former possibility is unlikely in the light of the existence of these modes even with the free-slip boundaries and makes other explanations more likely. The option to differentiate between effects caused by boundary interactions and ones caused by internal flow effects is one of the possible application for system with wholly different boundaries and understanding of it can be widened in further studies.

\subsubsection{Triad resonances}

The possibility of a triad resonance is a long discussed detail for axisymmetric containers: After [Malkus, 1968] had observed a wavelike instability inside a precessing spheroidal cavity, the exact cause for this was unknown to that time. A much more smooth flow was expected as formulated by [Stewartson and Roberts, 1963], more alike to the Poincaré flow [Poincaré, 1910]. At first, [Busse, 1968] deduced, that a differential rotation, induced by the boundary condition onto a Poincaré-flow, was causing the instability, until [Kerswell, 1993] formulated an alternative explanation: The spin-over mode is strained and couples with two inertial modes in a resonant effect, which subsequently tend to col- 
lapse into more disordered states. Something similar was predicted by [Mahalov, 1993] for a precessing, cylindrical annulus. One important feature of this form of triadic resonance is the difference of the azimuthal wave numbers of one between the both inertial waves, caused by the coupling with the spin-over mode with the same wave number $m=1$. The frequencies of the modes have to show equivalently a difference corresponding to the frequency of the spin-over mode, $\omega_{D}$. In sec. 3.5 it is shown, that in the cube modes with exactly these features exist. For $\mathrm{Ek}=1.0 \cdot 10^{-4}$ and $2.5 \cdot 10^{-4}$ triad resonances with azimuthal wave number pairs of $(1,2)$ and $(4,5)$ were found, with matching frequencies. Interestingly, in axisymmetric geometries, a dependence of the axial wave numbers to the spin-over mode is also necessary, but to this point could not be directly seen in the cube. This may be depending on a more complex structure alongside the axial direction, which at least show similar columnar shape in this direction as in other geometries [Lorenzani and Tilgner, 2003]. While analytical modal decomposition was not possible due to the unknown structure in cuboids geometries, the visual cues were unambiguous. In combination with the spectral analysis of the frequencies of the modes, this work confirms the existence of triad resonance due to precessing forces, comparable to triad resonances in other geometries like cylindrical annuli [Lin et al., 2014], full cylinders [Lagrange, Romain et al., 2009, Lagrange et al., 2011, Giesecke et al., 2015], and spheroidal shells [Lorenzani and Tilgner, 2001, Lorenzani and Tilgner, 2003]. The latter never was able to achieve a ratio of antisymmetric energy to kinetic energy $\frac{E_{a}}{E_{k i n}}$ above $10^{-2}$, while in the cube $\frac{E_{a}}{E_{k i n}}=0.17$ at $\Omega_{P}=-0.008$ is possible. This shows the ability of the cube to develop stronger instabilities in opposition to axisymmetric container which are more aligned to the effects of rotation. The capacity to use these instabilities to generate stronger dynamos is discussed in sec. 7.2.

\subsection{Kinematic Dynamo}

Many kinematic dynamos with acceptable critical numbers are found in chapter 4 for a wide range of different values of Ek and $\Omega_{P}$. The different classes of hydrodynamic effects categorized in chapter 3 played a huge role here. The triad resonance of sec. 3.5 present a mechanism to produce dynamo action at an acceptable magnetic Prandtl number. This is shown with the comparably low magnetic Reynolds numbers for the (1,2)-triad at $\mathrm{Ek}=1.0 \cdot 10^{-4}$ and $\Omega_{P}=-0.008$ with $\mathrm{Rm}_{\mathrm{c}, \mathrm{a}}=220$ and at $\mathrm{Ek}=2.5 \cdot 10^{-4}$ and $\Omega_{P}=-0.045$ with $\mathrm{Rm}_{\mathrm{c}, \mathrm{a}}=155$. The antisymmetric magnetic Reynolds number couples directly the generation of the magnetic field with the motion of the modes of triadic 
resonance and gives a clear picture of its potency but the core issue here is the feasibility to induce this motion. The transferred amount of energy from the underlying mode of the triad resonance to the antisymmetric modes has to be maximized for experimental setups to be effective. As discussed in 7.1.3 the ratio between total kinetic energy and antisymmetric energy $\frac{E_{a}}{E_{k i n}}$ never exceeds 0.2 , most cases even are below 0.1 . One most promising possibility is to find reasonable modifications of the container able to enhance the instability. In this work this was exemplary done by using cuboids as containers. These were selected for the especially strong tendency to instability of their ellipsoid counterparts and therefor may induce a stronger antisymmetric motion supporting the dynamo effect. Interestingly, this is the case with the critical full-flow magnetic Reynolds number $\mathrm{Rm}_{\mathrm{c}, \mathrm{kin}}$, so the dynamo effect improves against it in the cube, minimizing the necessary flow to induce the instability and subsequently reduce the power necessary to keep the container rotating in a hypothetical experimental setup. However, the effect on this critical number only reduces it to a third and with $\mathrm{Rm}_{\mathrm{c}, \mathrm{kin}}=2022$ at $\Omega_{P}=$ -0.1 and $\mathrm{Ek}=2.5 \cdot 10^{-4}$ it is still ten times above the best values of $\mathrm{Rm}_{\mathrm{c}, \mathrm{a}}$. For the rotational magnetic Reynolds number $\mathrm{Rm}_{\mathrm{c}, \text { rot }}$ depending on the rotation speed of the container $u=\left(\widetilde{\omega}_{D}+\widetilde{\Omega}_{P} \cos \alpha\right) \frac{L}{2}$ there is no difference between cube and cuboid containers for a fixed Ek, so for both cases the container has to rotate with the same speed to induce a dynamo. Most limiting for triad resonances as possible cause of dynamo effects is the limitation to relatively high Ekman numbers: For Ek $<1.0$. $10^{-4}$ no triads were observed in the system, thus restricting the capability to predict the efficiency of the triad resonance to generate magnetic fields for even lower Ekman numbers. Nevertheless [Giesecke et al., 2015] and [Lin et al., 2015] showed the existence of triads at lower Ekman numbers for cylinders and spheres respectively, so this effect may be relevant further on.

The influence of axisymmetric modes on the generation of magnetic fields is apparent in this work. As long as the axisymmetric modes are time independent and strong, the critical Reynolds number $\mathrm{Rm}_{\mathrm{c} \text {,rot }}$ is small in comparison to the other noted phases or when no axisymmetric modes exist as can be seen in fig. 7.1 for Ekman numbers between $5.0 \cdot 10^{-5}$ and $3.0 \cdot 10^{-4}$. Any instability of the axisymmetric modes inhibits the capacity to generate magnetic fields as for the unstable cases $\mathrm{Rm}_{\mathrm{c}, \text { rot }}$ varies only on a small range for differing $\Gamma_{0}$ and with no direct dependency. The influence and origin of these dynamics are not yet completely discovered and may be the focus of further investigations. As explained in sec. 7.1.2, the no-slip computer simulations, that were additionally done for comparison purposes, show stable modes at an Ekman number, where free-slip already 


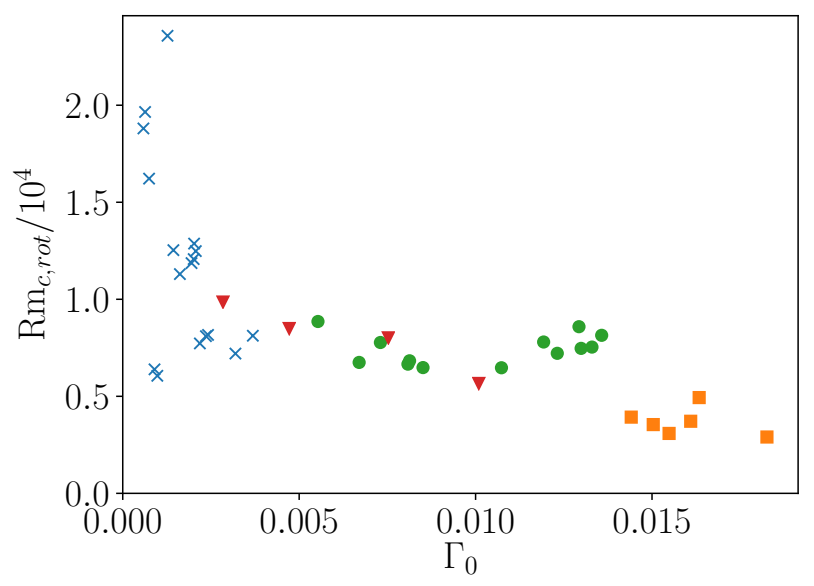

Figure 7.1: $\operatorname{Rm}_{\mathrm{c} \text {,rot }}\left(\Gamma_{0}\right)$ for the different phases of axisymmetric modes taken from parameter pairs of $\Omega_{P}$ from -0.2 up to -0.01 and Ek from $5.0 \cdot 10^{-5}$ to $3.0 \cdot 10^{-4}$ : time independent stable (squares), oscillating (circles), chaotic breakdown of (triangles) and non-existent modes (crosses).

induced oscillating modes, so the dynamo effect by axisymmetric modes possibly extend to lower values of Ek. Since further no-slip simulations are numerically unfeasible, no kinematic dynamo simulations were done to examine this aspect. However, the existence of stable axisymmetric modes with free-slip boundary condition and their effectiveness in dynamo action rules out viscous coupling with the boundary to be the particular effect in conjunction to the work of [Giesecke et al., 2018] which see an enhancement of the dynamo effect by axisymmetric modes with no-slip boundary condition. In contrast to the triadic resonance and the single vortex state, the axisymmetric modes are mainly connected to a laminar flow and the oscillations found in this work diminish the dynamo effect.

Neither triadic resonance nor axisymmetric modes show any promise at low Ekman numbers to be able to induce the dynamo effect in a fluid. Both effects vanish in the cubic confinement for Ek $<1.0 \cdot 10^{-4}$, so no reliable prediction of it for even lower Ekman numbers than $1.0 \cdot 10^{-5}$ can be done although they are effective for higher Ekman numbers. The single vortex state, however, does get stronger at decreasing Ek especially below $1.0 \cdot 10^{-4}$. This work did achieve to establish a connection between dynamo effect and this state for $\mathrm{Ek}=1.0 \cdot 10^{-5}$. The vortex decreases the critical magnetic Reynolds number in comparison to flows without it at the same Ekman number. Similar effects were found in numerical simulations for few parameter combinations in Ref. [Lin et al., 2016] 
for a spherical container: Three large vortex structures establish there and influence the magnetic field in a similar way to the single vortex state in this work. This reinforces the importance of possible vortex structures beyond cuboid geometries for containers. However, the broad range of values found by this simulation groundwork gives rise to more opportunities to examine the link between them. The actual quantitative relation between the vortex and the lowering of the critical magnetic Reynolds number can not be determined yet, since the measurement by $R_{\text {vortex }}$ is only able to qualitatively identify the single vortex state. For a more quantitative approach the notion of energy bound inside the vortex must be further developed. The best $\mathrm{Rm}_{\mathrm{c}}$ achieved at $\mathrm{Ek}=1.0 \cdot 10^{-5}$ and $\Omega_{P}=-0.02$ is high as opposed to the minimal values for $\mathrm{Ek} \geq 1.0 \cdot 10^{-4}$. The single vortex state may nevertheless improve these values for even lower Ekman numbers and be of interest in further examination of the flow.

The overall increase in the critical magnetic Reynolds number by decreasing Ekman number is accompanied by a decrease of the dissipation length $L_{D}$. Here the turbulent fluctuations broaden their spatial spectrum and induce more small scale movements into the flow. The increase of $\mathrm{Rm}_{\mathrm{c}}$ may be caused by a subsequent strong increase of magnetic eddy diffusivity while the magnetic field generation is not developing in the same strength. This is a restriction any experimental setup has to overcome. In the characteristic units used here the precessional dynamo setup built at the DRESDyn facility [Stefani et al., 2012, Stefani et al., 2015] will only produce $\mathrm{Rm}_{\text {rot }}$ below 1420 . Only the very low critical Reynolds number caused by the triadic resonance at $\mathrm{Ek}=$ $1.0 \cdot 10^{-4}$ and $\Omega_{P}=-0.008$ does come near this threshold. However, with $\mathrm{Ek} \approx 10^{-8}$ the DRESDyn setup will be four magnitudes below the Ekman number of this instability and no prediction for lower numbers with triadic resonance exist. The single vortex state is at this point the most promising flow to support the dynamo effect but further investigations into its structure and behavior at decreasing Ek is necessary. It is to conclude that the increase in eddy diffusivity is the main hindrance for very low Ek and in future the challenge is to find flow structure and container shapes, that will be able to counteract it to generate realistic dynamos.

\subsection{Herzenberg Dynamo}

As noticed in chapter 6, the used Herzenberg-like setup does not result in critical magnetic Prandtl numbers beneath those calculated by simple containers of similar extent. 
The most successful result was achieved by the $(1,0.1,1)_{H^{-}}$-setup at Ek $=5.0 \cdot 10^{-4}$; $\Omega_{P}=-0.35$, but there was no sign, that the rotor configuration was critical for the effect, since the second run with $\mathrm{Ek}=1.0 \cdot 10^{-4} ; \Omega_{P}=-0.1$ did not repeat the effect. Also, this setup was never expected in respect to Herzenberg's theory [Herzenberg, 1958]. The effect on other setups with differing containers and differing rotation axis was limited from start, as the containers as stand-alone cuboids had fitting critical magnetic Prandtl numbers on itself. Any possible effect of the Herzenberg-like setup had to improve significantly beyond these values. At none of the chosen parameters such an effect was noticed. This may have various reasons, one is, that this setup never followed exactly the setup presented by [Herzenberg, 1958] or [Brandenburg et al., 1998]. While Brandenburg showed, that the ratio of radius to distance $\frac{a}{d}$ does not have to be small like formulated by Herzenberg, they used a large periodic box to approximate the unbounded domain of Herzenberg's asymptotic theory. Here, on the other hand, this was not possible by means of the simulation code, which does not allow for a domain outside the fluid and limits the magnetic field with the pseudo-vacuum described in section 2.2.1, so that the fluid representing the rotor directly ends at the boundaries of the system. Possible modifications here would be implementing of more immersed boundaries on all sides of the container to simulate a larger resting domain around the rotor, but diminishing the efficiency of the computer calculations by adding additionally points to be simulated. Boundary conditions also limit the accuracy at another point: The velocity field at the inner boundary changes abruptly because of the stress-free flow implemented there. While this poses no problem at the outer boundaries, the ongoing magnetic field at the inner ones must be calculated by the discrete change in velocity. Here, the fourth order differences scheme is inappropriate to access results of a needed accuracy. Other boundary conditions like no-slip conditions would improve on that although they will reduce again the efficiency of the simulations like explained in section 2.2. Another way would be to arrange a similar 'soft' transition like Brandenburg used between the rigid spheres and the resting domain to avoid discrete transitions. Especially as replacement of the no-less 'unphysical' stress-free conditions could this be a good choice. The main restriction remaining is of course the selection of angles between the rotation axes. In contrast to the preceding setups with fixed rotation axes like the experiment by [Lowes and Wilkinson, 1963, Lowes and Wilkinson, 1968], here it was bound to the rotation axes given by flows at fixed precession. Only good predictions of the behavior of the flow inside each container can lead to different angles, like it was done by combining results of chapters 3 and 5 . But even here, it is limited to the maximum difference of the 
fluid axis, that two flows would reach under influence of the same precession. Of course, in this simulation, it would be possible to change the rotation axis $\boldsymbol{\omega}_{D}$ of the whole container only inside $C_{1}$ and $C_{2}$ so that it would seem that both containers rotate around different angles, changing the flow to include a greater range of possible angles between rotors. But this would be only possible because of the mantle system as reference system and would not be possible to be transferred into physical experiments. A lot of possible optimizations and improvements seem possible to adapt this simulation code to conduct simulations in Herzenberg-like setups, but this first examination does not predict any easy configurations, that would immediately improve a realistic experimental setup to produce reasonable dynamos. 


\section{Appendix}

\section{List of Symbols}

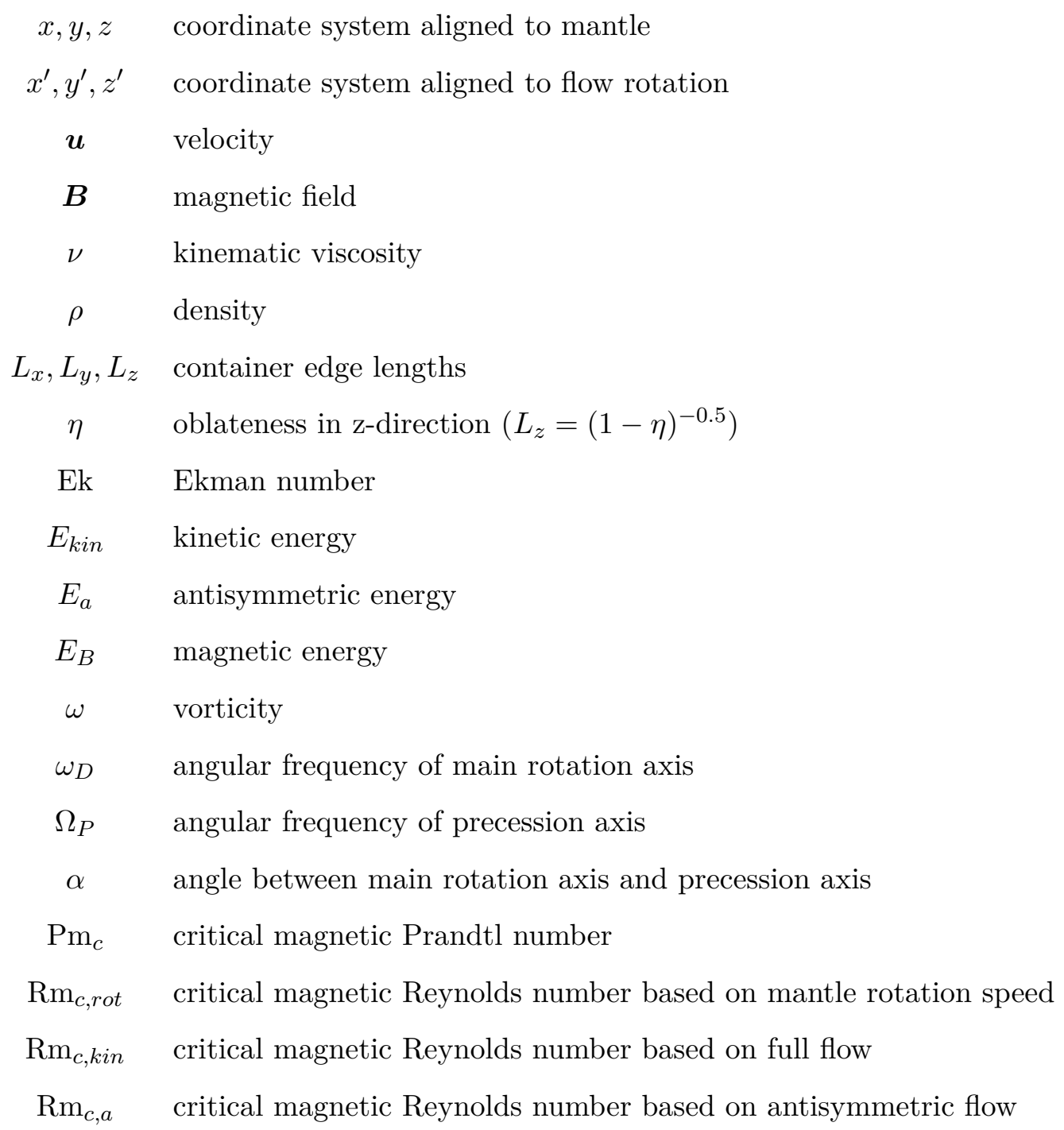




\section{Quantities}

Quantities of a cube with $L=1$ at $\mathrm{Ek}=1.0 \cdot 10^{-3}, \alpha=60^{\circ}$ averaged over time:

$\begin{array}{ccccc}\Omega_{P} & E_{k i n} & E_{a} & D & \mathrm{Pm}_{c} \\ -0.30 & 5.96 \mathrm{E}-02 & 2.13 \mathrm{E}-15 & 3.20 \mathrm{E}+00 & 1.07 \mathrm{E}+01 \\ -0.29 & 5.90 \mathrm{E}-02 & 3.04 \mathrm{E}-13 & 3.16 \mathrm{E}+00 & - \\ -0.28 & 5.83 \mathrm{E}-02 & 7.47 \mathrm{E}-14 & 3.13 \mathrm{E}+00 & 1.13 \mathrm{E}+01 \\ -0.27 & 5.77 \mathrm{E}-02 & 4.23 \mathrm{E}-14 & 3.10 \mathrm{E}+00 & 1.53 \mathrm{E}+01 \\ -0.26 & 5.72 \mathrm{E}-02 & 1.26 \mathrm{E}-13 & 3.09 \mathrm{E}+00 & 8.77 \mathrm{E}+00 \\ -0.25 & 5.67 \mathrm{E}-02 & 1.63 \mathrm{E}-14 & 3.08 \mathrm{E}+00 & 7.74 \mathrm{E}+00 \\ -0.24 & 5.63 \mathrm{E}-02 & 2.41 \mathrm{E}-13 & 3.09 \mathrm{E}+00 & 8.18 \mathrm{E}+00 \\ -0.23 & 5.58 \mathrm{E}-02 & 3.11 \mathrm{E}-14 & 3.12 \mathrm{E}+00 & 1.64 \mathrm{E}+01 \\ -0.22 & 5.50 \mathrm{E}-02 & 2.31 \mathrm{E}-14 & 3.13 \mathrm{E}+00 & 1.40 \mathrm{E}+01 \\ -0.21 & 5.35 \mathrm{E}-02 & 1.04 \mathrm{E}-14 & 3.09 \mathrm{E}+00 & - \\ -0.20 & 5.14 \mathrm{E}-02 & 9.75 \mathrm{E}-15 & 2.96 \mathrm{E}+00 & 1.43 \mathrm{E}+01 \\ -0.19 & 4.87 \mathrm{E}-02 & 1.01 \mathrm{E}-14 & 2.76 \mathrm{E}+00 & - \\ -0.18 & 4.55 \mathrm{E}-02 & 1.00 \mathrm{E}-14 & 2.51 \mathrm{E}+00 & - \\ -0.17 & 4.07 \mathrm{E}-02 & 6.33 \mathrm{E}-04 & 2.32 \mathrm{E}+00 & - \\ -0.16 & 3.91 \mathrm{E}-02 & 6.52 \mathrm{E}-04 & 2.25 \mathrm{E}+00 & 4.13 \mathrm{E}+01 \\ -0.15 & 3.74 \mathrm{E}-02 & 6.33 \mathrm{E}-04 & 2.17 \mathrm{E}+00 & - \\ -0.14 & 3.59 \mathrm{E}-02 & 6.79 \mathrm{E}-04 & 2.11 \mathrm{E}+00 & 2.58 \mathrm{E}+01 \\ -0.13 & 3.42 \mathrm{E}-02 & 6.92 \mathrm{E}-04 & 2.02 \mathrm{E}+00 & - \\ -0.12 & 3.23 \mathrm{E}-02 & 6.54 \mathrm{E}-04 & 1.90 \mathrm{E}+00 & 1.46 \mathrm{E}+01 \\ -0.11 & 3.03 \mathrm{E}-02 & 6.02 \mathrm{E}-04 & 1.76 \mathrm{E}+00 & 1.13 \mathrm{E}+01 \\ -0.10 & 2.92 \mathrm{E}-02 & 7.68 \mathrm{E}-15 & 1.48 \mathrm{E}+00 & - \\ -0.09 & 2.77 \mathrm{E}-02 & 5.30 \mathrm{E}-15 & 1.41 \mathrm{E}+00 & 2.24 \mathrm{E}+01 \\ -0.08 & 2.62 \mathrm{E}-02 & 5.88 \mathrm{E}-14 & 1.33 \mathrm{E}+00 & - \\ -0.07 & 2.46 \mathrm{E}-02 & 6.08 \mathrm{E}-14 & 1.25 \mathrm{E}+00 & - \\ -0.06 & 2.29 \mathrm{E}-02 & 7.93 \mathrm{E}-12 & 1.17 \mathrm{E}+00 & - \\ -0.05 & 2.11 \mathrm{E}-02 & 1.87 \mathrm{E}-15 & 1.09 \mathrm{E}+00 & -\end{array}$




$\begin{array}{ccccc}-0.04 & 1.90 \mathrm{E}-02 & 1.32 \mathrm{E}-11 & 9.99 \mathrm{E}-01 & - \\ -0.03 & 1.64 \mathrm{E}-02 & 1.16 \mathrm{E}-15 & 8.88 \mathrm{E}-01 & - \\ -0.02 & 1.23 \mathrm{E}-02 & 3.49 \mathrm{E}-12 & 6.83 \mathrm{E}-01 & -\end{array}$

Quantities of a cube with $L=1$ at $\mathrm{Ek}=2.5 \cdot 10^{-4}, \alpha=60^{\circ}$ averaged over time:

$\begin{array}{ccccc}\Omega_{P} & E_{k i n} & E_{a} & D & \mathrm{Pm}_{c} \\ -0.30 & 6.58 \mathrm{E}-02 & 4.84 \mathrm{E}-04 & 4.41 \mathrm{E}+00 & 6.24 \mathrm{E}+00 \\ -0.26 & 6.40 \mathrm{E}-02 & 4.74 \mathrm{E}-04 & 4.34 \mathrm{E}+00 & 5.55 \mathrm{E}+00 \\ -0.25 & 6.36 \mathrm{E}-02 & 4.81 \mathrm{E}-04 & 4.34 \mathrm{E}+00 & - \\ -0.24 & 6.34 \mathrm{E}-02 & 5.45 \mathrm{E}-04 & 4.36 \mathrm{E}+00 & 4.71 \mathrm{E}+00 \\ -0.22 & 6.33 \mathrm{E}-02 & 6.39 \mathrm{E}-04 & 4.46 \mathrm{E}+00 & 4.48 \mathrm{E}+00 \\ -0.21 & 6.34 \mathrm{E}-02 & 7.72 \mathrm{E}-04 & 4.55 \mathrm{E}+00 & - \\ -0.20 & 6.34 \mathrm{E}-02 & 8.29 \mathrm{E}-04 & 4.60 \mathrm{E}+00 & - \\ -0.19 & 6.34 \mathrm{E}-02 & 7.90 \mathrm{E}-04 & 4.62 \mathrm{E}+00 & 3.60 \mathrm{E}+00 \\ -0.18 & 5.98 \mathrm{E}-02 & 6.77 \mathrm{E}-04 & 4.82 \mathrm{E}+00 & 1.96 \mathrm{E}+00 \\ -0.17 & 6.00 \mathrm{E}-02 & 7.51 \mathrm{E}-04 & 4.90 \mathrm{E}+00 & 1.77 \mathrm{E}+00 \\ -0.16 & 5.98 \mathrm{E}-02 & 8.04 \mathrm{E}-04 & 4.90 \mathrm{E}+00 & 1.54 \mathrm{E}+00 \\ -0.15 & 5.94 \mathrm{E}-02 & 7.99 \mathrm{E}-04 & 4.93 \mathrm{E}+00 & 1.86 \mathrm{E}+00 \\ -0.14 & 5.89 \mathrm{E}-02 & 7.70 \mathrm{E}-04 & 4.94 \mathrm{E}+00 & 2.47 \mathrm{E}+00 \\ -0.13 & 5.54 \mathrm{E}-02 & 7.95 \mathrm{E}-04 & 4.63 \mathrm{E}+00 & 3.77 \mathrm{E}+00 \\ -0.12 & 5.49 \mathrm{E}-02 & 6.44 \mathrm{E}-04 & 4.68 \mathrm{E}+00 & 4.07 \mathrm{E}+00 \\ -0.11 & 5.31 \mathrm{E}-02 & 7.26 \mathrm{E}-04 & 4.58 \mathrm{E}+00 & 4.29 \mathrm{E}+00 \\ -0.10 & 5.21 \mathrm{E}-02 & 4.46 \mathrm{E}-04 & 4.58 \mathrm{E}+00 & 3.90 \mathrm{E}+00 \\ -0.09 & 5.10 \mathrm{E}-02 & 2.80 \mathrm{E}-04 & 4.51 \mathrm{E}+00 & 3.74 \mathrm{E}+00 \\ -0.083 & 4.93 \mathrm{E}-02 & 2.92 \mathrm{E}-04 & 4.49 \mathrm{E}+00 & - \\ -0.08 & 3.15 \mathrm{E}-02 & 4.33 \mathrm{E}-04 & 2.14 \mathrm{E}+00 & 3.86 \mathrm{E}+00 \\ -0.06 & 2.67 \mathrm{E}-02 & 1.86 \mathrm{E}-04 & 1.69 \mathrm{E}+00 & 3.69 \mathrm{E}+00 \\ -0.05 & 2.46 \mathrm{E}-02 & 8.38 \mathrm{E}-05 & 1.54 \mathrm{E}+00 & 3.19 \mathrm{E}+00\end{array}$




$$
\begin{array}{ccccc}
-0.0475 & 2.40 \mathrm{E}-02 & 7.76 \mathrm{E}-05 & 1.48 \mathrm{E}+00 & - \\
-0.045 & 2.35 \mathrm{E}-02 & 6.26 \mathrm{E}-05 & 1.49 \mathrm{E}+00 & 3.46 \mathrm{E}+00 \\
-0.0425 & 2.29 \mathrm{E}-02 & 1.84 \mathrm{E}-09 & 1.48 \mathrm{E}+00 & - \\
-0.02 & 1.73 \mathrm{E}-02 & 2.09 \mathrm{E}-04 & 1.08 \mathrm{E}+00 & 9.83 \mathrm{E}+00
\end{array}
$$

Quantities of a cube with $L=1$ at $\mathrm{Ek}=2.5 \cdot 10^{-4}, \alpha=60^{\circ}$ averaged over time while decreasing $\Omega_{P}$ from -0.008 to -0.04 :

$\begin{array}{cccc}\Omega_{P} & E_{k i n} & E_{a} & D \\ -0.040 & 2.21 \mathrm{E}-02 & 8.93 \mathrm{E}-08 & 1.36 \mathrm{E}+00 \\ -0.036 & 2.12 \mathrm{E}-02 & 1.09 \mathrm{E}-06 & 1.30 \mathrm{E}+00 \\ -0.032 & 2.04 \mathrm{E}-02 & 2.31 \mathrm{E}-04 & 1.26 \mathrm{E}+00 \\ -0.028 & 1.93 \mathrm{E}-02 & 2.22 \mathrm{E}-04 & 1.20 \mathrm{E}+00 \\ -0.024 & 1.83 \mathrm{E}-02 & 2.18 \mathrm{E}-04 & 1.13 \mathrm{E}+00 \\ -0.020 & 1.73 \mathrm{E}-02 & 2.14 \mathrm{E}-04 & 1.07 \mathrm{E}+00 \\ -0.016 & 1.62 \mathrm{E}-02 & 1.97 \mathrm{E}-04 & 1.01 \mathrm{E}+00 \\ -0.012 & 9.03 \mathrm{E}-04 & 6.37 \mathrm{E}-17 & 6.28 \mathrm{E}-02\end{array}$

Quantities of a cube with $L=1$ at $\mathrm{Ek}=2.5 \cdot 10^{-4}, \alpha=60^{\circ}$ averaged over time while increasing $\Omega_{P}$ from -0.04 to -0.008 :

$\begin{array}{cccc}\Omega_{P} & E_{k i n} & E_{a} & D \\ -0.036 & 2.11 \mathrm{E}-02 & 2.05 \mathrm{E}-15 & 1.30 \mathrm{E}+00 \\ -0.032 & 2.01 \mathrm{E}-02 & 3.15 \mathrm{E}-15 & 1.26 \mathrm{E}+00 \\ -0.028 & 1.92 \mathrm{E}-02 & 5.76 \mathrm{E}-15 & 1.22 \mathrm{E}+00 \\ -0.024 & 1.83 \mathrm{E}-02 & 2.17 \mathrm{E}-04 & 1.14 \mathrm{E}+00 \\ -0.020 & 1.73 \mathrm{E}-02 & 2.14 \mathrm{E}-04 & 1.07 \mathrm{E}+00 \\ -0.016 & 1.62 \mathrm{E}-02 & 1.98 \mathrm{E}-04 & 1.01 \mathrm{E}+00\end{array}$




$$
\begin{array}{rrrr}
-0.012 & 1.45 \mathrm{E}-02 & 1.60 \mathrm{E}-04 & 8.95 \mathrm{E}-01 \\
-0.008 & 3.26 \mathrm{E}-04 & 2.36 \mathrm{E}-17 & 2.33 \mathrm{E}-02
\end{array}
$$

\begin{tabular}{|c|c|c|c|c|}
\hline$\Omega_{P}$ & $E_{k i n}$ & $E_{a}$ & $D$ & $\mathrm{~m}_{c}$ \\
\hline-0.30 & $6.80 \mathrm{E}-02$ & $4.65 \mathrm{E}-04$ & $5.20 \mathrm{E}+00$ & $2.51 \mathrm{E}+00$ \\
\hline-0.25 & 6.63E-02 & 4.92E-04 & $5.12 \mathrm{E}+00$ & - \\
\hline-0.23 & $6.57 \mathrm{E}-02$ & $5.16 \mathrm{E}-04$ & $5.08 \mathrm{E}+00$ & - \\
\hline-0.22 & $6.56 \mathrm{E}-02$ & $5.25 \mathrm{E}-04$ & $5.11 \mathrm{E}+00$ & - \\
\hline-0.21 & $6.54 \mathrm{E}-02$ & $5.19 \mathrm{E}-04$ & $5.08 \mathrm{E}+00$ & - \\
\hline-0.20 & $6.54 \mathrm{E}-02$ & $5.73 \mathrm{E}-04$ & $5.13 \mathrm{E}+00$ & - \\
\hline-0.19 & $6.57 \mathrm{E}-02$ & $6.14 \mathrm{E}-04$ & $5.16 \mathrm{E}+00$ & - \\
\hline-0.18 & $6.55 \mathrm{E}-02$ & 6.37E-04 & $5.19 \mathrm{E}+00$ & - \\
\hline-0.17 & $6.56 \mathrm{E}-02$ & $6.92 \mathrm{E}-04$ & $5.21 \mathrm{E}+00$ & - \\
\hline-0.16 & $6.55 \mathrm{E}-02$ & $7.18 \mathrm{E}-04$ & $5.19 \mathrm{E}+00$ & $1.62 \mathrm{E}+00$ \\
\hline-0.15 & $6.55 \mathrm{E}-02$ & 7.32E-04 & $5.24 \mathrm{E}+00$ & - \\
\hline-0.14 & $6.41 \mathrm{E}-02$ & 7.99E-04 & $5.20 \mathrm{E}+00$ & $1.70 \mathrm{E}+00$ \\
\hline-0.13 & $6.36 \mathrm{E}-02$ & 8.17E-04 & $5.19 \mathrm{E}+00$ & $1.60 \mathrm{E}+00$ \\
\hline-0.12 & 6.30E-02 & 6.69E-04 & $5.23 \mathrm{E}+00$ & $1.23 \mathrm{E}+00$ \\
\hline-0.11 & 6.30E-02 & $5.78 \mathrm{E}-04$ & $5.23 \mathrm{E}+00$ & $1.33 \mathrm{E}+00$ \\
\hline-0.10 & $6.26 \mathrm{E}-02$ & $6.01 \mathrm{E}-04$ & $5.23 \mathrm{E}+00$ & $1.30 \mathrm{E}+00$ \\
\hline-0.09 & $6.21 \mathrm{E}-02$ & $6.14 \mathrm{E}-04$ & $5.22 \mathrm{E}+00$ & $1.37 \mathrm{E}+00$ \\
\hline-0.08 & $6.11 \mathrm{E}-02$ & $6.06 \mathrm{E}-04$ & $5.16 \mathrm{E}+00$ & - \\
\hline-0.07 & $5.94 \mathrm{E}-02$ & $5.98 \mathrm{E}-04$ & $5.13 \mathrm{E}+00$ & $1.55 \mathrm{E}+00$ \\
\hline-0.06 & $5.77 \mathrm{E}-02$ & $5.65 \mathrm{E}-04$ & $5.07 \mathrm{E}+00$ & - \\
\hline-0.05 & $5.36 \mathrm{E}-02$ & $6.43 \mathrm{E}-04$ & $4.93 \mathrm{E}+00$ & $1.77 \mathrm{E}+00$ \\
\hline 0.045 & $5.15 \mathrm{E}-02$ & 8.71E-04 & $4.86 \mathrm{E}+00$ & - \\
\hline 0 & $97 \mathrm{E}-02$ & $9.32 \mathrm{E}-04$ & $4.81 \mathrm{E}+00$ & $\mathrm{E}+$ \\
\hline
\end{tabular}

Quantities of a cube with $L=1$ at $\mathrm{Ek}=1.0 \cdot 10^{-4}, \alpha=60^{\circ}$ averaged over time: 


$\begin{array}{ccccc}-0.038 & 2.59 \mathrm{E}-02 & 6.75 \mathrm{E}-04 & 1.94 \mathrm{E}+00 & - \\ -0.035 & 2.48 \mathrm{E}-02 & 6.89 \mathrm{E}-04 & 3.51 \mathrm{E}+00 & - \\ -0.03 & 2.34 \mathrm{E}-02 & 8.45 \mathrm{E}-04 & 1.75 \mathrm{E}+00 & 4.72 \mathrm{E}+00 \\ -0.02 & 1.93 \mathrm{E}-02 & 1.41 \mathrm{E}-03 & 1.64 \mathrm{E}+00 & 2.41 \mathrm{E}+00 \\ -0.01 & 5.66 \mathrm{E}-04 & 6.78 \mathrm{E}-16 & 4.19 \mathrm{E}-02 & - \\ -0.008 & 1.09 \mathrm{E}-02 & 1.82 \mathrm{E}-03 & 8.41 \mathrm{E}-01 & 3.64 \mathrm{E}-01 \\ -0.005 & 1.17 \mathrm{E}-04 & 1.58 \mathrm{E}-16 & 9.02 \mathrm{E}-03 & -\end{array}$

Quantities of a cube with $L=1$ at $\mathrm{Ek}=1.0 \cdot 10^{-4}, \alpha=60^{\circ}$ averaged over time while decreasing $\Omega_{P}$ from 0.0 to -0.04 :

$\begin{array}{cccc}\Omega_{P} & E_{k i n} & E_{a} & D \\ -0.040 & 2.60 \mathrm{E}-02 & 6.11 \mathrm{E}-04 & 1.89 \mathrm{E}+00 \\ -0.036 & 2.49 \mathrm{E}-02 & 6.84 \mathrm{E}-04 & 1.81 \mathrm{E}+00 \\ -0.032 & 2.41 \mathrm{E}-02 & 7.77 \mathrm{E}-04 & 1.77 \mathrm{E}+00 \\ -0.028 & 2.28 \mathrm{E}-02 & 9.12 \mathrm{E}-04 & 1.71 \mathrm{E}+00 \\ -0.024 & 2.10 \mathrm{E}-02 & 1.19 \mathrm{E}-03 & 1.73 \mathrm{E}+00 \\ -0.020 & 1.93 \mathrm{E}-02 & 1.43 \mathrm{E}-03 & 1.70 \mathrm{E}+00 \\ -0.018 & 1.85 \mathrm{E}-02 & 1.54 \mathrm{E}-03 & 1.59 \mathrm{E}+00 \\ -0.016 & 2.04 \mathrm{E}-03 & 1.16 \mathrm{E}-05 & 1.63 \mathrm{E}-01 \\ -0.012 & 8.47 \mathrm{E}-04 & 2.37 \mathrm{E}-11 & 7.44 \mathrm{E}-02 \\ -0.008 & 3.31 \mathrm{E}-04 & 8.20 \mathrm{E}-16 & 2.49 \mathrm{E}-02 \\ -0.004 & 7.34 \mathrm{E}-05 & 7.44 \mathrm{E}-17 & 5.62 \mathrm{E}-03\end{array}$

Quantities of a cube with $L=1$ at $\mathrm{Ek}=1.0 \cdot 10^{-4}, \alpha=60^{\circ}$ averaged over time while increasing $\Omega_{P}$ from -0.04 to -0.004 : 


$\begin{array}{cccc}\Omega_{P} & E_{k i n} & E_{a} & D \\ -0.036 & 2.50 \mathrm{E}-02 & 6.70 \mathrm{E}-04 & 1.81 \mathrm{E}+00 \\ -0.032 & 2.41 \mathrm{E}-02 & 7.78 \mathrm{E}-04 & 1.81 \mathrm{E}+00 \\ -0.028 & 2.27 \mathrm{E}-02 & 9.04 \mathrm{E}-04 & 1.71 \mathrm{E}+00 \\ -0.024 & 2.10 \mathrm{E}-02 & 1.15 \mathrm{E}-03 & 1.70 \mathrm{E}+00 \\ -0.020 & 1.92 \mathrm{E}-02 & 1.45 \mathrm{E}-03 & 1.68 \mathrm{E}+00 \\ -0.016 & 1.75 \mathrm{E}-02 & 1.57 \mathrm{E}-03 & 1.41 \mathrm{E}+00 \\ -0.012 & 1.47 \mathrm{E}-02 & 1.79 \mathrm{E}-03 & 1.17 \mathrm{E}+00 \\ -0.008 & 1.09 \mathrm{E}-02 & 1.82 \mathrm{E}-03 & 8.41 \mathrm{E}-01 \\ -0.004 & 1.76 \mathrm{E}-04 & 4.83 \mathrm{E}-08 & 6.07 \mathrm{E}-03\end{array}$

Quantities of a cube with $L=1$ at $\mathrm{Ek}=1.0 \cdot 10^{-5}, \alpha=60^{\circ}$ averaged over time:

$\begin{array}{ccccc}\Omega_{P} & E_{k i n} & E_{a} & D & \mathrm{Pm}_{c} \\ -0.30 & 7.36 \mathrm{E}-02 & 2.06 \mathrm{E}-04 & 1.46 \mathrm{E}+01 & 4.17 \mathrm{E}-01 \\ -0.16 & 7.44 \mathrm{E}-02 & 3.80 \mathrm{E}-04 & 3.15 \mathrm{E}+01 & 3.33 \mathrm{E}-01 \\ -0.12 & 7.30 \mathrm{E}-02 & 3.46 \mathrm{E}-04 & 2.65 \mathrm{E}+01 & - \\ -0.10 & 7.32 \mathrm{E}-02 & 4.04 \mathrm{E}-04 & 3.06 \mathrm{E}+01 & 3.24 \mathrm{E}-01 \\ -0.09 & 7.27 \mathrm{E}-02 & 4.24 \mathrm{E}-04 & 3.18 \mathrm{E}+01 & - \\ -0.08 & 7.12 \mathrm{E}-02 & 4.58 \mathrm{E}-04 & 3.45 \mathrm{E}+01 & - \\ -0.07 & 6.88 \mathrm{E}-02 & 4.62 \mathrm{E}-04 & 3.34 \mathrm{E}+01 & 3.22 \mathrm{E}-01 \\ -0.06 & 6.42 \mathrm{E}-02 & 4.45 \mathrm{E}-04 & 3.43 \mathrm{E}+01 & - \\ -0.05 & 6.12 \mathrm{E}-02 & 4.09 \mathrm{E}-04 & 3.11 \mathrm{E}+01 & 3.64 \mathrm{E}-01 \\ -0.04 & 5.79 \mathrm{E}-02 & 3.78 \mathrm{E}-04 & 2.69 \mathrm{E}+01 & 3.77 \mathrm{E}-01 \\ -0.03 & 5.68 \mathrm{E}-02 & 7.79 \mathrm{E}-04 & 2.97 \mathrm{E}+01 & 3.23 \mathrm{E}-01 \\ -0.02 & 5.31 \mathrm{E}-02 & 5.39 \mathrm{E}-04 & 2.14 \mathrm{E}+01 & 2.34 \mathrm{E}-01 \\ -0.01 & 4.78 \mathrm{E}-02 & 4.06 \mathrm{E}-04 & 1.09 \mathrm{E}+01 & 4.21 \mathrm{E}-01\end{array}$


Quantities of a cube with $L=1$ at $\Omega_{P}=-0.02, \alpha=60^{\circ}$ averaged over time:

$\begin{array}{ccccc}\text { Ek } & E_{k i n} & E_{a} & D & \mathrm{Pm}_{c} \\ 1.00 \mathrm{E}-05 & 5.31 \mathrm{E}-02 & 5.39 \mathrm{E}-04 & 2.14 \mathrm{E}+01 & 2.34 \mathrm{E}-01 \\ 2.50 \mathrm{E}-05 & 1.89 \mathrm{E}-02 & 9.98 \mathrm{E}-04 & 8.27 \mathrm{E}+00 & - \\ 5.00 \mathrm{E}-05 & 1.95 \mathrm{E}-02 & 1.20 \mathrm{E}-03 & 1.12 \mathrm{E}+01 & 2.73 \mathrm{E}+00 \\ 1.00 \mathrm{E}-04 & 1.93 \mathrm{E}-02 & 1.41 \mathrm{E}-03 & 1.64 \mathrm{E}+01 & 2.41 \mathrm{E}+00 \\ 1.50 \mathrm{E}-04 & 1.80 \mathrm{E}-02 & 1.43 \mathrm{E}-03 & 1.88 \mathrm{E}+01 & 1.81 \mathrm{E}+00 \\ 2.00 \mathrm{E}-04 & 1.62 \mathrm{E}-02 & 4.61 \mathrm{E}-04 & 2.08 \mathrm{E}+01 & 3.79 \mathrm{E}+00 \\ 2.50 \mathrm{E}-04 & 1.73 \mathrm{E}-02 & 2.09 \mathrm{E}-04 & 2.69 \mathrm{E}+01 & 9.83 \mathrm{E}+00 \\ 3.00 \mathrm{E}-04 & 1.69 \mathrm{E}-02 & 2.34 \mathrm{E}-04 & 2.58 \mathrm{E}+01 & 1.13 \mathrm{E}+01\end{array}$

Quantities of a cube with $L=1$ at $\Omega_{P}=-0.05, \alpha=60^{\circ}$ averaged over time:

$\begin{array}{ccccc}\mathrm{Ek} & E_{k i n} & E_{a} & D & \mathrm{Pm}_{c} \\ 1.00 \mathrm{E}-05 & 6.12 \mathrm{E}-02 & 4.09 \mathrm{E}-04 & 3.11 \mathrm{E}+01 & 3.64 \mathrm{E}-01 \\ 5.00 \mathrm{E}-05 & 6.24 \mathrm{E}-02 & 4.43 \mathrm{E}-04 & 2.86 \mathrm{E}+01 & 1.16 \mathrm{E}+00 \\ 7.50 \mathrm{E}-05 & 5.77 \mathrm{E}-02 & 5.74 \mathrm{E}-04 & 3.93 \mathrm{E}+01 & - \\ 1.00 \mathrm{E}-04 & 5.36 \mathrm{E}-02 & 6.43 \mathrm{E}-04 & 4.93 \mathrm{E}+01 & 1.77 \mathrm{E}+00 \\ 1.50 \mathrm{E}-04 & 4.77 \mathrm{E}-02 & 1.13 \mathrm{E}-03 & 6.87 \mathrm{E}+01 & 3.85 \mathrm{E}+00 \\ 2.00 \mathrm{E}-04 & 2.43 \mathrm{E}-02 & 2.84 \mathrm{E}-04 & 3.04 \mathrm{E}+01 & 2.56 \mathrm{E}+00 \\ 2.50 \mathrm{E}-04 & 2.46 \mathrm{E}-02 & 8.38 \mathrm{E}-05 & 3.85 \mathrm{E}+01 & 3.19 \mathrm{E}+00 \\ 3.00 \mathrm{E}-04 & 2.42 \mathrm{E}-02 & 1.02 \mathrm{E}-04 & 4.55 \mathrm{E}+01 & 3.63 \mathrm{E}+00\end{array}$

Quantities of a cube with $L=1$ at $\Omega_{P}=-0.1, \alpha=60^{\circ}$ averaged over time:

Ek $\quad E_{k i n} \quad E_{a} \quad D \quad \mathrm{Pm}_{c}$

$1.00 \mathrm{E}-05 \quad 7.32 \mathrm{E}-02 \quad 4.04 \mathrm{E}-04 \quad 3.06 \mathrm{E}+01 \quad 3.24 \mathrm{E}-01$ 


$\begin{array}{ccccc}1.50 \mathrm{E}-05 & 7.22 \mathrm{E}-02 & 4.02 \mathrm{E}-04 & 3.02 \mathrm{E}+01 & - \\ 2.00 \mathrm{E}-05 & 7.12 \mathrm{E}-02 & 4.20 \mathrm{E}-04 & 2.99 \mathrm{E}+01 & - \\ 2.50 \mathrm{E}-05 & 7.00 \mathrm{E}-02 & 4.14 \mathrm{E}-04 & 3.01 \mathrm{E}+01 & 5.84 \mathrm{E}-01 \\ 5.00 \mathrm{E}-05 & 6.55 \mathrm{E}-02 & 6.87 \mathrm{E}-04 & 2.84 \mathrm{E}+01 & 9.86 \mathrm{E}-01 \\ 7.50 \mathrm{E}-05 & 6.40 \mathrm{E}-02 & 5.85 \mathrm{E}-04 & 4.16 \mathrm{E}+01 & 1.21 \mathrm{E}+00 \\ 1.00 \mathrm{E}-04 & 6.26 \mathrm{E}-02 & 6.01 \mathrm{E}-04 & 5.23 \mathrm{E}+01 & 1.30 \mathrm{E}+00 \\ 1.50 \mathrm{E}-04 & 5.91 \mathrm{E}-02 & 5.05 \mathrm{E}-04 & 7.55 \mathrm{E}+01 & 1.94 \mathrm{E}+00 \\ 2.00 \mathrm{E}-04 & 5.56 \mathrm{E}-02 & 5.74 \mathrm{E}-04 & 9.58 \mathrm{E}+01 & 2.89 \mathrm{E}+00 \\ 2.50 \mathrm{E}-04 & 5.21 \mathrm{E}-02 & 4.46 \mathrm{E}-04 & 1.14 \mathrm{E}+02 & 3.90 \mathrm{E}+00 \\ 3.00 \mathrm{E}-04 & 3.90 \mathrm{E}-02 & 2.86 \mathrm{E}-04 & 8.49 \mathrm{E}+01 & 4.90 \mathrm{E}+00\end{array}$

Quantities of a cube with $L=1$ at $\Omega_{P}=-0.16, \alpha=60^{\circ}$ averaged over time:

$\begin{array}{ccccc}\mathrm{Ek} & E_{k i n} & E_{a} & D & \mathrm{Pm}_{c} \\ 1.00 \mathrm{E}-05 & 7.44 \mathrm{E}-02 & 3.80 \mathrm{E}-04 & 3.15 \mathrm{E}+01 & 3.33 \mathrm{E}-01 \\ 5.00 \mathrm{E}-05 & 6.68 \mathrm{E}-02 & 4.70 \mathrm{E}-04 & 2.92 \mathrm{E}+01 & 1.02 \mathrm{E}+00 \\ 1.00 \mathrm{E}-04 & 6.55 \mathrm{E}-02 & 7.18 \mathrm{E}-04 & 5.19 \mathrm{E}+01 & 1.62 \mathrm{E}+00 \\ 1.50 \mathrm{E}-04 & 6.40 \mathrm{E}-02 & 7.81 \mathrm{E}-04 & 7.41 \mathrm{E}+01 & 2.44 \mathrm{E}+00 \\ 2.00 \mathrm{E}-04 & 6.11 \mathrm{E}-02 & 8.01 \mathrm{E}-04 & 9.82 \mathrm{E}+01 & 2.27 \mathrm{E}+00 \\ 2.50 \mathrm{E}-04 & 5.98 \mathrm{E}-02 & 8.04 \mathrm{E}-04 & 1.23 \mathrm{E}+02 & 1.54 \mathrm{E}+00 \\ 3.00 \mathrm{E}-04 & 5.84 \mathrm{E}-02 & 8.45 \mathrm{E}-04 & 1.44 \mathrm{E}+02 & 1.74 \mathrm{E}+00\end{array}$

Quantities of a cube with $L=1$ at $\Omega_{P}=-0.3, \alpha=60^{\circ}$ averaged over time:

$\begin{array}{ccccc}\text { Ek } & E_{k i n} & E_{a} & D & \mathrm{Pm}_{c} \\ 1.00 \mathrm{E}-05 & 7.36 \mathrm{E}-02 & 2.06 \mathrm{E}-04 & 1.46 \mathrm{E}+01 & 4.17 \mathrm{E}-01 \\ 2.00 \mathrm{E}-05 & 7.05 \mathrm{E}-02 & 1.99 \mathrm{E}-04 & 1.50 \mathrm{E}+01 & -\end{array}$




$$
\begin{array}{lllll}
5.00 \mathrm{E}-05 & 6.92 \mathrm{E}-02 & 3.49 \mathrm{E}-04 & 2.95 \mathrm{E}+01 & 1.62 \mathrm{E}+00 \\
1.00 \mathrm{E}-04 & 6.80 \mathrm{E}-02 & 4.65 \mathrm{E}-04 & 5.20 \mathrm{E}+01 & 2.51 \mathrm{E}+00 \\
1.50 \mathrm{E}-04 & 6.71 \mathrm{E}-02 & 5.09 \mathrm{E}-04 & 7.31 \mathrm{E}+01 & 3.39 \mathrm{E}+00 \\
2.00 \mathrm{E}-04 & 6.65 \mathrm{E}-02 & 4.94 \mathrm{E}-04 & 9.26 \mathrm{E}+01 & 4.75 \mathrm{E}+00 \\
2.50 \mathrm{E}-04 & 6.58 \mathrm{E}-02 & 4.84 \mathrm{E}-04 & 1.10 \mathrm{E}+02 & 6.24 \mathrm{E}+00 \\
3.00 \mathrm{E}-04 & 6.50 \mathrm{E}-02 & 4.66 \mathrm{E}-04 & 1.27 \mathrm{E}+02 & 7.72 \mathrm{E}+00
\end{array}
$$

Quantities of a cuboid with $L_{x}=L_{y}=1$ and $\eta=-0.7$ at $\mathrm{Ek}=2.5 \cdot 10^{-4}, \alpha=60^{\circ}$ averaged over time:

$\begin{array}{cccc}\Omega_{P} & E_{k i n} & E_{a} & D \\ -0.26 & 5.47 \mathrm{E}-02 & 1.41 \mathrm{E}-03 & 3.08 \mathrm{E}+01 \\ -0.20 & 4.75 \mathrm{E}-02 & 1.38 \mathrm{E}-03 & 1.82 \mathrm{E}+01 \\ -0.18 & 4.40 \mathrm{E}-02 & 1.22 \mathrm{E}-03 & 1.47 \mathrm{E}+01 \\ -0.16 & 4.10 \mathrm{E}-02 & 1.18 \mathrm{E}-03 & 3.74 \mathrm{E}+00 \\ -0.14 & 3.79 \mathrm{E}-02 & 1.18 \mathrm{E}-03 & 4.37 \mathrm{E}+00 \\ -0.13 & 1.23 \mathrm{E}-02 & 1.02 \mathrm{E}-03 & 8.78 \mathrm{E}+00 \\ -0.12 & 9.21 \mathrm{E}-03 & 8.97 \mathrm{E}-04 & 2.12 \mathrm{E}+00 \\ -0.11 & 7.41 \mathrm{E}-03 & 7.48 \mathrm{E}-04 & 1.04 \mathrm{E}+00 \\ -0.10 & 5.71 \mathrm{E}-03 & 5.76 \mathrm{E}-04 & 1.46 \mathrm{E}+00 \\ -0.09 & 5.20 \mathrm{E}-03 & 4.81 \mathrm{E}-04 & 7.95 \mathrm{E}-01 \\ -0.08 & 3.05 \mathrm{E}-03 & 2.67 \mathrm{E}-04 & 3.24 \mathrm{E}+00 \\ -0.06 & 1.32 \mathrm{E}-03 & 3.86 \mathrm{E}-16 & 5.01 \mathrm{E}-01 \\ -0.04 & 5.98 \mathrm{E}-04 & 1.66 \mathrm{E}-16 & 2.25 \mathrm{E}-01 \\ -0.03 & 3.41 \mathrm{E}-04 & 6.44 \mathrm{E}-17 & 2.08 \mathrm{E}-02 \\ -0.02 & 1.54 \mathrm{E}-04 & 3.24 \mathrm{E}-17 & 9.59 \mathrm{E}-03 \\ -0.015 & 8.71 \mathrm{E}-05 & 1.60 \mathrm{E}-17 & 5.49 \mathrm{E}-03 \\ -0.01 & 3.91 \mathrm{E}-05 & 1.56 \mathrm{E}-17 & 2.49 \mathrm{E}-03 \\ -0.005 & 9.85 \mathrm{E}-06 & 1.32 \mathrm{E}-17 & 6.33 \mathrm{E}-04\end{array}$


Quantities of a cuboid with $L_{x}=L_{y}=1$ and $\eta=-0.5$ at Ek $=2.5 \cdot 10^{-4}, \alpha=60^{\circ}$ averaged over time:

$\begin{array}{cccc}\Omega_{P} & E_{k i n} & E_{a} & D \\ -0.30 & 5.96 \mathrm{E}-02 & 9.78 \mathrm{E}-04 & 4.20 \mathrm{E}+00 \\ -0.25 & 5.42 \mathrm{E}-02 & 7.99 \mathrm{E}-04 & 3.95 \mathrm{E}+00 \\ -0.20 & 5.23 \mathrm{E}-02 & 9.57 \mathrm{E}-04 & 3.97 \mathrm{E}+00 \\ -0.15 & 4.30 \mathrm{E}-02 & 7.59 \mathrm{E}-04 & 3.33 \mathrm{E}+00 \\ -0.12 & 1.30 \mathrm{E}-02 & 6.16 \mathrm{E}-07 & 9.26 \mathrm{E}-01 \\ -0.10 & 9.73 \mathrm{E}-03 & 2.52 \mathrm{E}-04 & 6.47 \mathrm{E}-01 \\ -0.08 & 6.65 \mathrm{E}-03 & 1.23 \mathrm{E}-04 & 4.34 \mathrm{E}-01 \\ -0.05 & 2.55 \mathrm{E}-03 & 3.18 \mathrm{E}-14 & 1.51 \mathrm{E}-01\end{array}$

Quantities of a cuboid with $L_{x}=L_{y}=1$ and $\eta=-0.7$ at $\Omega_{P}=-0.1, \alpha=60^{\circ}$ averaged over time:

$\begin{array}{ccccc}\text { Ek } & E_{k i n} & E_{a} & D & \mathrm{Pm}_{c} \\ 1.00 \mathrm{E}-05 & 7.94 \mathrm{E}-03 & 6.00 \mathrm{E}-04 & 4.99 \mathrm{E}+00 & 3.40 \mathrm{E}-01 \\ 5.00 \mathrm{E}-05 & 5.41 \mathrm{E}-02 & 6.42 \mathrm{E}-04 & 2.67 \mathrm{E}+01 & 9.06 \mathrm{E}-01 \\ 1.00 \mathrm{E}-04 & 1.25 \mathrm{E}-02 & 1.29 \mathrm{E}-03 & 1.03 \mathrm{E}+01 & 1.15 \mathrm{E}+00 \\ 1.50 \mathrm{E}-04 & 7.91 \mathrm{E}-03 & 8.43 \mathrm{E}-04 & 1.00 \mathrm{E}+01 & 2.03 \mathrm{E}+00 \\ 2.00 \mathrm{E}-04 & 6.57 \mathrm{E}-03 & 6.96 \mathrm{E}-04 & 1.01 \mathrm{E}+01 & 2.48 \mathrm{E}+00 \\ 2.50 \mathrm{E}-04 & 5.71 \mathrm{E}-03 & 5.76 \mathrm{E}-04 & 1.03 \mathrm{E}+01 & 4.73 \mathrm{E}+00 \\ 3.00 \mathrm{E}-04 & 5.30 \mathrm{E}-03 & 5.29 \mathrm{E}-04 & 1.11 \mathrm{E}+01 & 5.52 \mathrm{E}+00\end{array}$




\section{Bibliography}

[Berhanu et al., 2007] Berhanu, M., Monchaux, R., Fauve, S., Mordant, N., Pétrélis, F., Chiffaudel, A., Daviaud, F., Dubrulle, B., Marié, L., Ravelet, F., et al. (2007). Magnetic field reversals in an experimental turbulent dynamo. EPL (Europhysics Letters), 77(5):59001.

[Braginsky and Roberts, 1995] Braginsky, S. I. and Roberts, P. H. (1995). Equations governing convection in earth's core and the geodynamo. Geophysical \& Astrophysical Fluid Dynamics, 79(1-4):1-97.

[Brandenburg et al., 1998] Brandenburg, A., Moss, D., and Soward, A. (1998). New results for the herzenberg dynamo: steady and oscillatory solutions. In Proceedings of the Royal Society of London A: Mathematical, Physical and Engineering Sciences, volume 454, pages 1283-1300. The Royal Society.

[Bullard, 1949] Bullard, E. C. (1949). The magnetic field within the earth. In Proc. $R$. Soc. London, Ser. A, volume 197, pages 433-453.

[Busse, 1968] Busse, F. H. (1968). Steady fluid flow in a precessing spheroidal shell. Journal of Fluid Mechanics, 33(4):739-751.

[Cappanera et al., 2016] Cappanera, L., Guermond, J.-L., Léorat, J., and Nore, C. (2016). Two spinning ways for precession dynamo. Physical Review E, 93(4):043113.

[Cébron and Hollerbach, 2014] Cébron, D. and Hollerbach, R. (2014). Tidally driven dynamos in a rotating sphere. The Astrophysical Journal Letters, 789(1):L25.

[Chong et al., 1990] Chong, M. S., Perry, A. E., and Cantwell, B. J. (1990). A general classification of three-dimensional flow fields. Physics of Fluids A: Fluid Dynamics, $2(5): 765-777$.

[Chorin, 1967] Chorin, A. J. (1967). A numerical method for solving incompressible viscous flow problems. Journal of Computational Physics, 2(1):12-26. 
[Dolginov and Urpin, 1979] Dolginov, A. Z. and Urpin, V. A. (1979). The inductive generation of the magnetic field in binary systems. Astronomy and Astrophysics, 79(1-2):60-69.

[Dudley, 1989] Dudley, J. (1989). Time-dependent kinematic dynamos with stationary flows. Proceedings of the Royal Society of London A: Mathematical, Physical and Engineering Sciences, 425(1869):407-429.

[Ekman, 1905] Ekman, V. W. (1905). On the influence of the earth's rotation on oceancurrents. Ark. Mat. Astr. Fys., 2(11).

[Gailitis et al., 2000] Gailitis, A., Lielausis, O., Dement'ev, S., Platacis, E., Cifersons, A., Gerbeth, G., Gundrum, T., Stefani, F., Christen, M., Hänel, H., et al. (2000). Detection of a flow induced magnetic field eigenmode in the riga dynamo facility. Physical Review Letters, 84(19):4365.

[Gans, 1971] Gans, R. F. (1971). On hydromagnetic precession in a cylinder. Journal of Fluid Mechanics, 45(1):111-130.

[Gibson, 1968a] Gibson, R. D. (1968a). The herzenberg dynamo. i. The Quarterly Journal of Mechanics and Applied Mathematics, 21(2):243-255.

[Gibson, 1968b] Gibson, R. D. (1968b). The herzenberg dynamo ii. The Quarterly Journal of Mechanics and Applied Mathematics, 21(2):257-267.

[Giesecke et al., 2015] Giesecke, A., Albrecht, T., Gundrum, T., Herault, J., and Stefani, F. (2015). Triadic resonances in nonlinear simulations of a fluid flow in a precessing cylinder. New Journal of Physics, 17(11):113044.

[Giesecke et al., 2018] Giesecke, A., Vogt, T., Gundrum, T., and Stefani, F. (2018). Nonlinear large scale flow in a precessing cylinder and its ability to drive dynamo action. Physical Review Letters, 120(2):024502.

[Goepfert and Tilgner, 2016] Goepfert, O. and Tilgner, A. (2016). Dynamos in precessing cubes. New Journal of Physics, 18(10):103019.

[Goepfert and Tilgner, ewed] Goepfert, O. and Tilgner, A. (reviewed). Mechanisms for magnetic field generation in precessing cubes. Geophysical \& Astrophysical Fluid Dynamics. 
[Greenspan, 1969] Greenspan, H. (1969). The Theory of Rotating Fluids. Cambridge Monographs on Mechanics and Applied Mathematics. At the University Press.

[Herault et al., 2015] Herault, J., Gundrum, T., Giesecke, A., and Stefani, F. (2015). Subcritical transition to turbulence of a precessing flow in a cylindrical vessel. Physics of Fluids, 27(12):124102.

[Herzenberg, 1958] Herzenberg, A. (1958). Geomagnetic dynamos. Philosophical Transactions of the Royal Society of London A: Mathematical, Physical and Engineering Sciences, 250(986):543-583.

[Kerswell, 1993] Kerswell, R. R. (1993). The instability of precessing flow. Geophysical Es Astrophysical Fluid Dynamics, 72(1-4):107-144.

[Kerswell, 1999] Kerswell, R. R. (1999). Secondary instabilities in rapidly rotating fluids: inertial wave breakdown. Journal of Fluid Mechanics, 382:283-306.

[Lagrange et al., 2008] Lagrange, R., Eloy, C., Nadal, F., and Meunier, P. (2008). Instability of a fluid inside a precessing cylinder. Physics of Fluids, 20(8):081701.

[Lagrange et al., 2011] Lagrange, R., Meunier, P., Nadal, F., and Eloy, C. (2011). Precessional instability of a fluid cylinder. Journal of Fluid Mechanics, 666:104-145.

[Lagrange, Romain et al., 2009] Lagrange, Romain, Meunier, Patrice, Eloy, Christophe, and Nadal, François (2009). Dynamics of a fluid inside a precessing cylinder. Mécanique 83 Industries, 10(3-4):187-194.

[Lin et al., 2015] Lin, Y., Marti, P., and Noir, J. (2015). Shear-driven parametric instability in a precessing sphere. Physics of Fluids, 27(4):--

[Lin et al., 2016] Lin, Y., Marti, P., Noir, J., and Jackson, A. (2016). Precession-driven dynamos in a full sphere and the role of large scale cyclonic vortices. Physics of Fluids, 28(6):066601.

[Lin et al., 2014] Lin, Y., Noir, J., and Jackson, A. (2014). Experimental study of fluid flows in a precessing cylindrical annulus. Physics of Fluids (1994-present), 26(4):-.

[Lorenzani, 2002] Lorenzani, S. (2002). Fluid instabilities in precessing ellipsoidal shells. $\mathrm{PhD}$ thesis, Georg-August-Universität Göttingen.

[Lorenzani and Tilgner, 2001] Lorenzani, S. and Tilgner, A. (2001). Fluid instabilities in precessing spheroidal cavities. Journal of Fluid Mechanics, 447:111-128. 
[Lorenzani and Tilgner, 2003] Lorenzani, S. and Tilgner, A. (2003). Inertial instabilities of fluid flow in precessing spheroidal shells. Journal of Fluid Mechanics, 492:363-380.

[Lowes and Wilkinson, 1963] Lowes, F. and Wilkinson, I. (1963). Geomagnetic dynamo: a laboratory model. Nature, 198(4886):1158-1160.

[Lowes and Wilkinson, 1968] Lowes, F. and Wilkinson, I. (1968). Geomagnetic dynamo: an improved laboratory model. Nature, 219(5155):717-718.

[Maas, 2003] Maas, L. R. (2003). On the amphidromic structure of inertial waves in a rectangular parallelepiped. Fluid Dynamics Research, 33(4):373-401.

[Mahalov, 1993] Mahalov, A. (1993). The instability of rotating fluid columns subjected to a weak external coriolis force. Physics of Fluids A: Fluid Dynamics, 5(4):891-900.

[Malkus, 1968] Malkus, W. (1968). Precession of the earth as the cause of geomagnetism: Experiments lend support to the proposal that precessional torques drive the earth's dynamo. Science, 160(3825):259-264.

[Manasseh, 1992] Manasseh, R. (1992). Breakdown regimes of inertia waves in a precessing cylinder. Journal of Fluid Mechanics, 243:261-296.

[Meunier et al., 2008] Meunier, P., Eloy, C., Lagrange, R., and Nadal, F. (2008). A rotating fluid cylinder subject to weak precession. Journal of Fluid Mechanics, 599:405440.

[Moffatt, 1978] Moffatt, H. K. (1978). Magnetic field generation in electrically conducting fluids. Cambridge University Press, Cambridge, London, New York, Melbourne.

[Monchaux et al., 2007] Monchaux, R., Berhanu, M., Bourgoin, M., Moulin, M., Odier, P., Pinton, J.-F., Volk, R., Fauve, S., Mordant, N., Pétrélis, F., et al. (2007). Generation of a magnetic field by dynamo action in a turbulent flow of liquid sodium. Physical review letters, 98(4):044502.

[Mouhali et al., 2012] Mouhali, W., Lehner, T., Léorat, J., and Vitry, R. (2012). Evidence for a cyclonic regime in a precessing cylindrical container. Experiments in fluids, 53(6):1693-1700.

[Müller et al., 2008] Müller, U., Stieglitz, R., Busse, F. H., and Tilgner, A. (2008). The karlsruhe two-scale dynamo experiment. Comptes Rendus Physique, 9(7):729-740. 
[Müller et al., 2006] Müller, U., Stieglitz, R., and Horanyi, S. (2006). Experiments at a two-scale dynamo test facility. Journal of Fluid Mechanics, 552:419-440.

[Nore et al., 2011] Nore, C., Léorat, J., Guermond, J.-L., and Luddens, F. (2011). Nonlinear dynamo action in a precessing cylindrical container. Physical Review E, 84(1):016317.

[Pétrélis et al., 2009] Pétrélis, F., Fauve, S., Dormy, E., and Valet, J.-P. (2009). Simple mechanism for reversals of earth's magnetic field. Physical review letters, 102(14):144503.

[Poincaré, 1910] Poincaré, H. (1910). Sur la précession des corps déformables. Bulletin Astronomique, Serie I, 27:321-356.

[Ponomarenko, 1973] Ponomarenko, Y. B. (1973). Theory of the hydromagnetic generator. Journal of Applied Mechanics and Technical Physics, 14(6):775-778.

[Ravelet et al., 2005] Ravelet, F., Chiffaudel, A., Daviaud, F., and Léorat, J. (2005). Toward an experimental von kármán dynamo: Numerical studies for an optimized design. Physics of fluids, 17(11):117104.

[Roberts, 1972] Roberts, G. O. (1972). Dynamo action of fluid motions with twodimensional periodicity. Phil. Trans. R. Soc. Lond. A, 271(1216):411-454.

[Stefani et al., 2017] Stefani, F., Albrecht, T., Arlt, R., Christen, M., Gailitis, A., Gellert, M., Giesecke, A., Goepfert, O., Herault, J., Kirillov, O., et al. (2017). Magnetic field dynamos and magnetically triggered flow instabilities. In IOP Conference Series: Materials Science and Engineering, volume 228, page 012002. IOP Publishing.

[Stefani et al., 2015] Stefani, F., Albrecht, T., Gerbeth, G., Giesecke, A., Gundrum, T., Herault, J., Nore, C., and Steglich, C. (2015). Towards a precession driven dynamo experiment. Magnetogydrodynamics, 51(2):275-284.

[Stefani et al., 2012] Stefani, F., Eckert, S., Gerbeth, G., Giesecke, A., Gundrum, T., Steglich, C., Weier, T., and Wustmann, B. (2012). Dresdyn-a new facility for mhd experiments with liquid sodium. Magnetohydrodynamics, 48(1):103-114.

[Stewartson and Roberts, 1963] Stewartson, K. and Roberts, P. (1963). On the motion of liquid in a spheroidal cavity of a precessing rigid body. Journal of fluid mechanics, $17(1): 1-20$. 
[Tilgner, 2005] Tilgner, A. (2005). Precession driven dynamos. Physics of Fluids, 17(3).

[Tilgner, 2012] Tilgner, A. (2012). Transitions in rapidly rotating convection driven dynamos. Physical review letters, 109(24):248501.

[Tilgner, 2014] Tilgner, A. (2014). Magnetic energy dissipation and mean magnetic field generation in planar convection-driven dynamos. Physical Review E, 90(1):013004.

[Tilgner and Busse, 2001] Tilgner, A. and Busse, F. (2001). Fluid flows in precessing spherical shells. Journal of Fluid Mechanics, 426:387-396.

[Vanyo et al., 1995] Vanyo, J., Wilde, P., Cardin, P., and Olson, P. (1995). Experiments on precessing flows in the earth's liquid core. Geophysical Journal International, 121(1):136-142.

[Vorobieff and Ecke, 1998] Vorobieff, P. and Ecke, R. E. (1998). Vortex structure in rotating rayleigh-benard convection. Physica D: Nonlinear Phenomena, 123(1-4):153160.

[Vorobieff and Ecke, 2002] Vorobieff, P. and Ecke, R. E. (2002). Turbulent rotating convection: an experimental study. Journal of Fluid Mechanics, 458:191-218.

[Wu and Roberts, 2009] Wu, C.-C. and Roberts, P. H. (2009). On a dynamo driven by topographic precession. Geophysical and Astrophysical Fluid Dynamics, 103(6):467501.

[Wu and Roberts, 2011] Wu, C.-C. and Roberts, P. H. (2011). High order instabilities of the poincaré solution for precessionally driven flow. Geophysical \&3 Astrophysical Fluid Dynamics, 105(2-3):287-303.

[Wu and Roberts, 2013] Wu, C.-C. and Roberts, P. H. (2013). On a dynamo driven topographically by longitudinal libration. Geophysical \& Astrophysical Fluid Dynamics, 107(1-2):20-44. 


\section{Acknowledgements}

First and foremost, I want to thank Prof. Dr. Tilgner for his time and constant support over the years. He always had a fitting piece of advice on hand for every problem and his guidance was always appreciated.

Furthermore, I would like to express my gratitude to Prof. Dr. Lube. He offered a lot of new perspectives on the possibilities of numeric work on numerous occasions and always bore with my at times physicist's attitude.

I am also grateful for all the help, input and warm welcome I received every time I visited DRESDyn. In particular, I would like to thank Dr. Frank Stefani for all the effort and interest in my work, Dr. Andre Giesecke for his support and advice in numerical matters, Thomas Gundrum for his many insights into the world of precession experiments and Johann Herault for sharing his ideas and thoughts.

This work would not exist if it were not for my parents Rosemarie and Reinhard. They selflessly helped me without a moment's thought whenever needed and I am so very lucky in being able to always count on their support.

I sincerely want to thank my colleagues and friends for all their support - be it in terms of proof-reading, stimulating discussions, technical support, sharing thoughts and ideas over coffee or lunch, participation in all of our shenanigans or bearing with my antics. With special thanks to Tristan Koppe, Julian Amani, Kevin Lüdemann, Matthias Kellner, Christoph Brüsewitz, Christa Gaißbichler and Cornelius Warner.

Thank You all for helping me making this possible! 


\section{Resume}

\section{Personal Data}

$\begin{array}{ll}\text { Name } & \text { Goepfert } \\ \text { Vorname } & \text { Oliver } \\ \text { Geburtsdatum } & 03.07 .1986 \\ \text { Geburtsort } & \text { Hannover } \\ \text { Staatsangehörigkeit } & \text { Deutsch } \\ \text { Familienstand } & \text { ledig }\end{array}$

\section{Educational Background}

$02.09 .1999-16.06 .2006$

$01.10 .2006-07.10 .2009$

$08.10 .2009-31.10 .2012$

01.10.2013 - heute

\author{
Abitur, Gymnasium Sarstedt, Sarstedt \\ Bachelor of Science, Georg-August-Universität Göttingen, \\ Göttingen \\ Kristallographische Simulationsrechnungen zu geordneten Po- \\ laronenphasen \\ Master of Science, Georg-August-Universität Göttingen, \\ Göttingen \\ Monte-Carlo-Simulation von Musterbildung und Abschat- \\ tungseffekten auf Si-Oberflächen unter senkrechtem Ionen- \\ strahlbeschuss und Codeposition \\ Promotion, Georg-August-Universität Göttingen, Göttingen
}

